\title{
With the body in mind : the role of exercise and acid- base balance in panic
}

Citation for published version (APA):

Esquivel, G. (2009). With the body in mind : the role of exercise and acid-base balance in panic. [Doctoral Thesis, Maastricht University]. Maastricht University. https://doi.org/10.26481/dis.20091014ge

Document status and date:

Published: 01/01/2009

DOI:

10.26481/dis.20091014ge

Document Version:

Publisher's PDF, also known as Version of record

\section{Please check the document version of this publication:}

- A submitted manuscript is the version of the article upon submission and before peer-review. There can be important differences between the submitted version and the official published version of record.

People interested in the research are advised to contact the author for the final version of the publication, or visit the DOI to the publisher's website.

- The final author version and the galley proof are versions of the publication after peer review.

- The final published version features the final layout of the paper including the volume, issue and page numbers.

Link to publication

\footnotetext{
General rights rights.

- You may freely distribute the URL identifying the publication in the public portal. please follow below link for the End User Agreement:

www.umlib.nl/taverne-license

Take down policy

If you believe that this document breaches copyright please contact us at:

repository@maastrichtuniversity.nl

providing details and we will investigate your claim.
}

Copyright and moral rights for the publications made accessible in the public portal are retained by the authors and/or other copyright owners and it is a condition of accessing publications that users recognise and abide by the legal requirements associated with these

- Users may download and print one copy of any publication from the public portal for the purpose of private study or research.

- You may not further distribute the material or use it for any profit-making activity or commercial gain

If the publication is distributed under the terms of Article $25 \mathrm{fa}$ of the Dutch Copyright Act, indicated by the "Taverne" license above, 


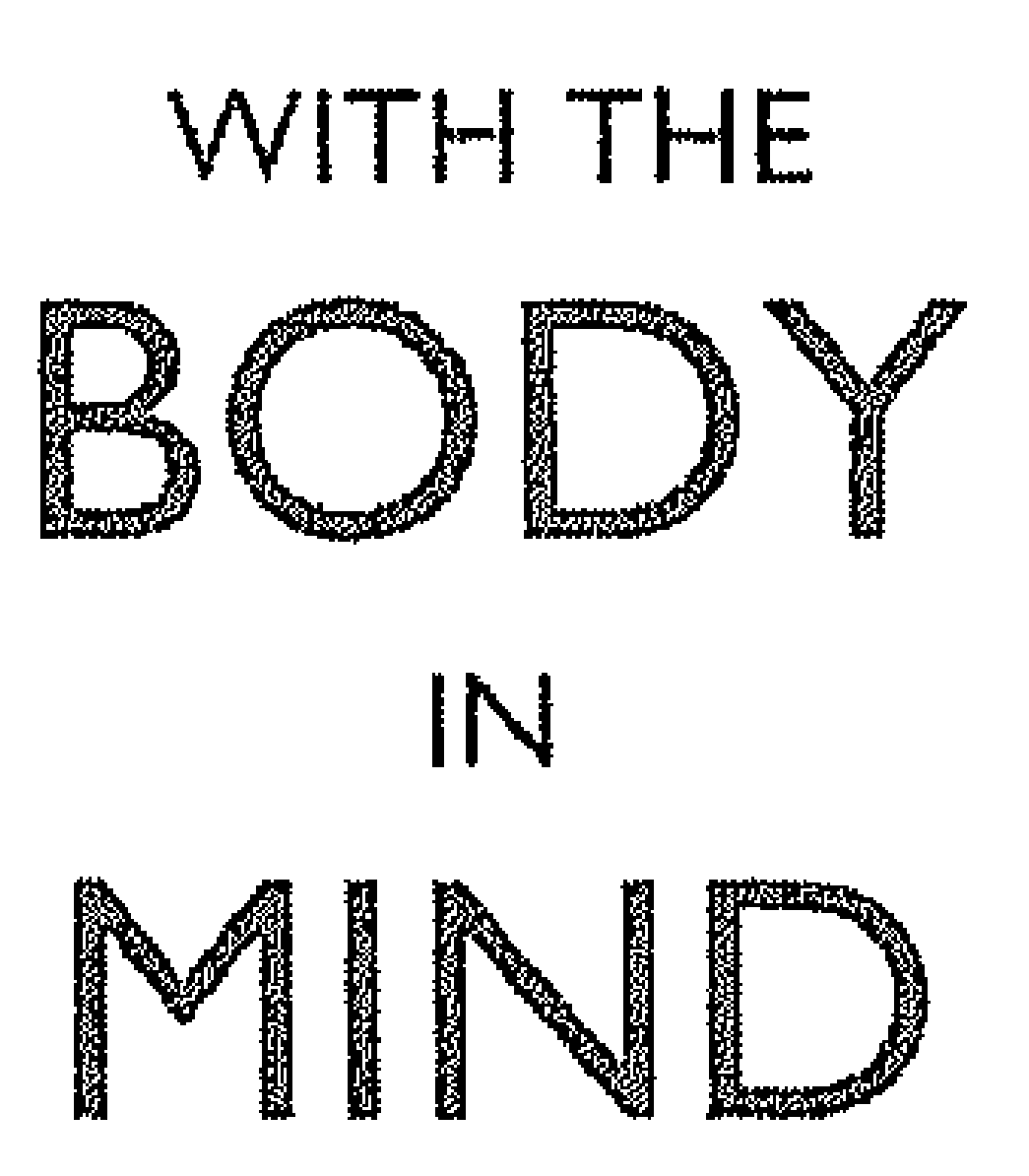

THE ROLE OF EXERCISE AND ACID-BASE BALANCE IN PANIC 
(c) Gabriel Esquivel, Maastricht 2009

Design: G. Esquivel

Print: Ridderprint, Ridderkerk

ISBN 978-90-5335-201-4 
to obtain the degree of DOCTOR at the Maastricht University on the authority of the Rector Magnificus, Prof. dr. G.P.M.F Mols in accordance with the decision of the Board of Deans, to be defended in public on Wednesday, the 14th of October 2009, at 12:00 hours 


\section{Supervisor:}

Prof. dr. E.J.L Griez

\section{Co-supervisor:}

Dr. K.R.J Schruers

\section{Assessment Committee:}

Prof.dr. H. Kuipers (chairman)

Dr. C. Henquet

Dr. G.P. Perna (Milan, Italy)

Dr. J.H. Prickaerts

Prof.dr. W. Riedel

Prof.dr. V. Visser-Vandewalle

The research presented in this thesis was conducted at the Academic Anxiety Center Maastricht, Mondriaan Zorggroup, School for Mental Health and Neuroscience, Maastricht University, Maastricht, The Netherlands and the Instituto Nacional de Psiquiatría Ramón de la Fuente Muñiz, División de Investigaciones Clínicas, Mexico City, Mexico.

This thesis was financially supported by CONACyT (Mexico). 
Para Elena y Wendolin 
Paranymphs:

Iván Abarca

Lies Goossens 


\section{Contents}

General overview

Chapter 1 Introduction

Part 1. Experimental models of panic 13

Part 2. The exercise-affect relationship 45

Chapter 2 The effects of acute exercise on 35\% CO2 induced panic in healthy volunteers

Chapter 3 Acute exercise reduces the effects of experimental panic provocation with 35\% CO2 in patients with panic disorder

Chapter 4 Effects of exercise on models of $\mathrm{CO} 2$ induced panic 77

Chapter 5 The effects of opioid receptor blockade on experimental panic provocation with $35 \% \mathrm{CO} 2$

Chapter 6 The association between 5-HTTLPR genotype and $\mathrm{CO} 2$ induced negative affectivity

Chapter 7 The relationship between habitual physical activity and symptom severity in panic disorder

Chapter 8 Acids in the brain: a factor in panic?

Summary and concluding remarks

Samenvatting en conclusies

Acknowledgments

Curriculum vitae

Publication list 
General overview 


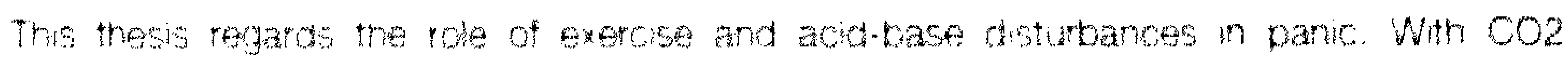

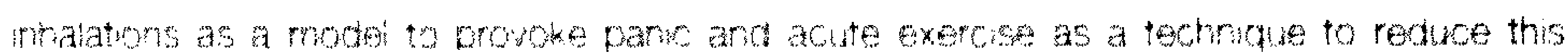

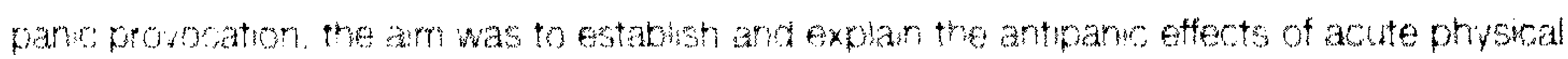

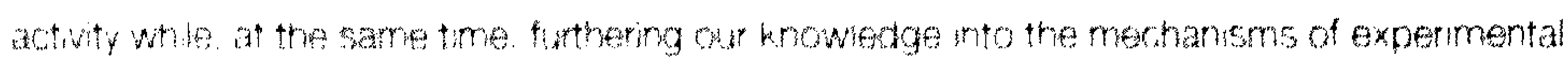
a.)

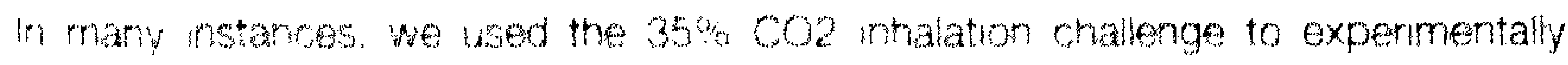

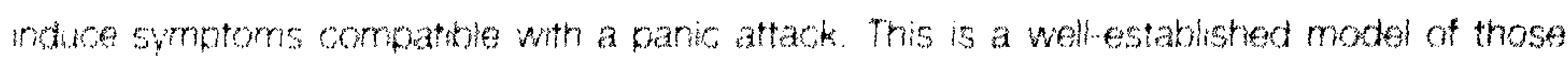

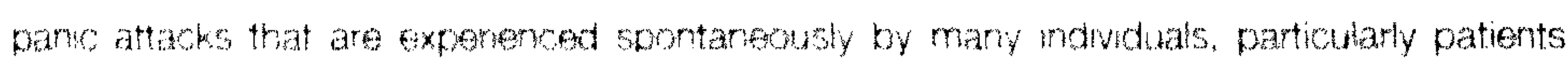

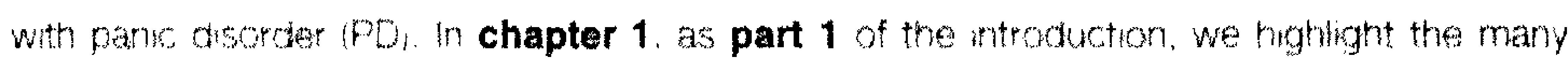
aspocts that ars revent to an adequate model of panc as a frame to discuss the mary different expermontat methods that have bosn chimed to provoke panic. As such. when the waldy of these dfferent temniques lie sodum lactate infusion: CO2 inhalation; voluntary

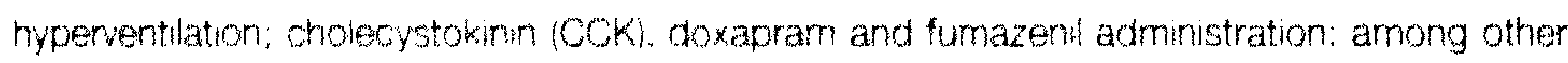
methods is reviewed. many issues can be examined. Beyond the aspects that are related to the validty of a model of panic. this part of the untroduction also describes techrical and physiological aspects as well as the putatwe mechanisms of action related to the different panic provocation techriques.

We used acute exercise as an antpanic agent. The increase of physical actuty in the form of regular exercise, an established heathy behavor, has ganed increasing interest as a form of therapy for affective disorders including depression and anxety. Also in chapter 1, as part 2 of our introduction, we reviewed the literature for which the use of exercise as an evidence-based therapy for affective disorders can be discussed. The level of evidence, from both epidemiotogical and experimental studies, is considered to support the idea of a positive relationship between levels of physical activity and relief from depressive and anxious symptoms. The potential mechanisms related to the effects of exercise and other relevant issues are abo discussed.

Previous literature had pointed to the fact that intense exercise, despite a sharp increase in both physical symptoms of arousal and endogenous lactate, is well tolerated by patients with panic disorder. We further hypothesized that intense exercise has antipanic properties. In the study described in chapter 2, as a first step, we tested this hypothesis in healthy volunteers. Participants were randomized to perform either moderate/hard or very-light exercise on a bicycle ergometer and afterwards subjected to a single $35 \% \mathrm{CO} 2$ challenge. For this challenge, subjects were asked to exhale to the maximum, to position the inhalation mask on their face and inhale their full capacity as quickly as possible, whereupon they had to hold their breath for 4 seconds before exhaling. The intensity of symptoms consistent with current nosological criteria for a panic attacks was measured before and after the $\mathrm{CO} 2$ challenge. The score increases in the measured symptoms produced by the challenge were used as main 


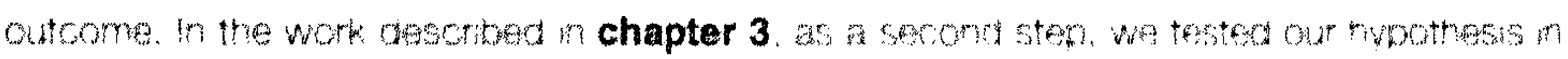

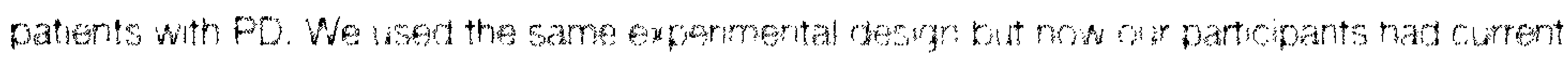

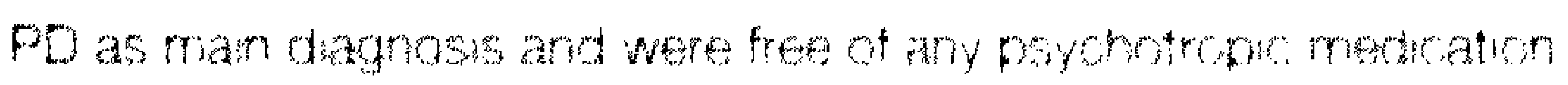

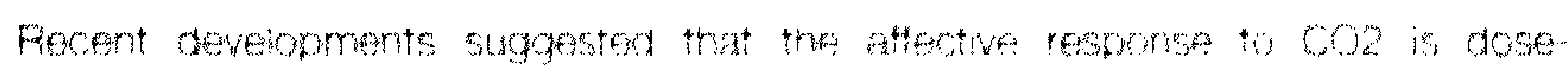

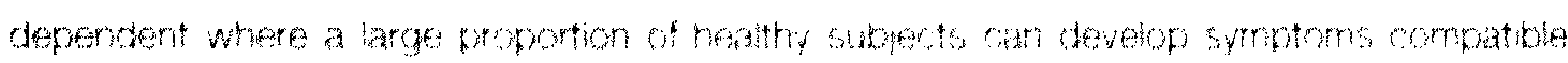

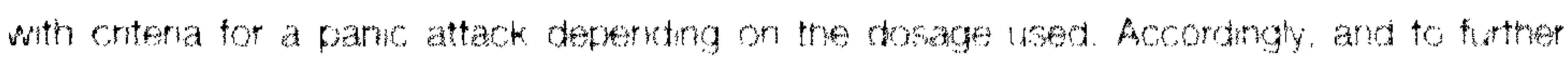

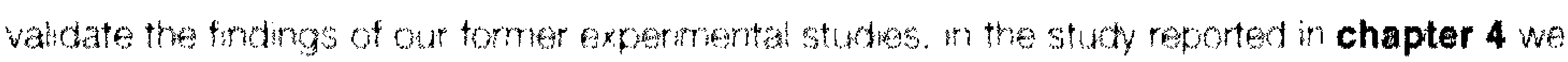

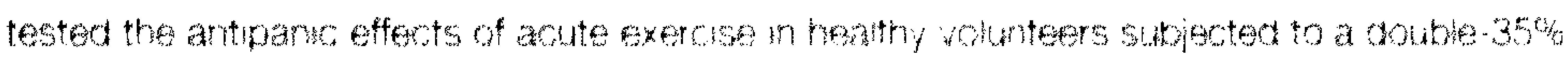

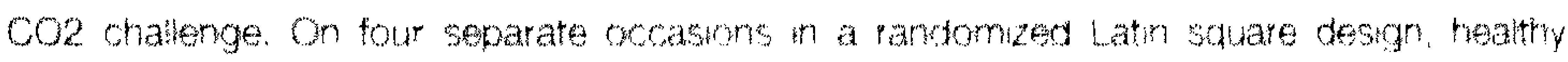

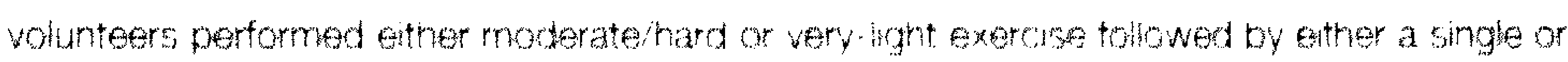

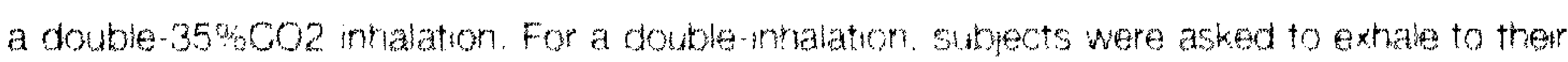

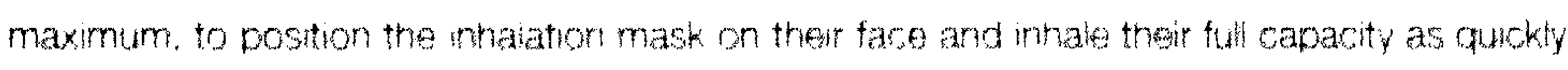
as possibse. Next they were to empty their ungs and refll them immediately with the gas mixture. Whareupon they had to hold them brath for 4 seconds before exhaing. Hare. we also used scome increases on measured symptoms of penio as man outcome. We hypothesized that exercise would show protective effects against the reactions to both 602 dosages.

As an attempt to explain the effects of acute exercise on 002 induced affectivity. we undertook studies desungd to manipuate some of the putative reurotransmiter systems that are invoved. The endorphrergic system has been a popular suspect underlyng the positive effects of exercise on mood and anxiety. Moreover, opoids are estabished anti-dyspnea compounds and recent literature has implicated endogenous opoid system as a moderating factor in the threshold for the development of a panc attack. Before probing the irvolvement of this system in the effects of acute exercise on the affectivity elicited by $\mathrm{CO} 2$. We planned to observe the effects of pharmacologicaly blocking the endogenous opioid system alone on this challenge. In chapter 5, we detat a study with heaithy volunteers in a double-blind. randomized, crossover design that oraly receved ether natrexone $50 \mathrm{mg}$ or placebo Natrexone is a long-acting pharmacologicat agent that blocks opioid receptors. Most subjects undertook a $35 \%$ CO2 double-inhalation one hour after pre-medication and a separate group did so after 5 hours. We predicted that the administration of naltrexone would increase the response to $\mathrm{CO2}$. Consistent with the methods of our lab. we used score increases in measured symptoms of panic as main outcome.

With basis on studies in animal models of acute intense physical activity and on established pharmacological antipanic treatment, the serotonergic system is another suspect implicated in the effects of acute exercise on $\mathrm{CO} 2$ induced panic. Furthermore, $\mathrm{CO}$-induced affectivity has shown to be moderated by pharmacological manipulation of 5-HT activity. Differences in serotonin $(5-4 T)$ activity may also naturally be caused by a functional polymorphism in the promoter region of the 5.HT transporter gene (serotonin transporter gene 
General overview

linked polymorphic region, 5-HTTLPR). In the study described in chapter 6, as part of a series of studies on both the underlying mechanisms of $\mathrm{CO} 2$ induced panic and also the acute antipanic effects of exercise, we examined the effect of 5-HTTLPR genotype in healthy volunteers undergoing different dosages of inhaled CO2. Ninety-six subjects performed a doubleinhalation of four mixtures containing respectively $0,9,17.5$ and $35 \% \mathrm{CO} 2$, following a randomized, double-blind, cross-over design. Genotype was determined using buccal cell material from all participants. We expected increases in measured symptoms of panic to be modulated by 5-HTTLPR genotype.

Outside the laboratory, does the engagement in regular exercise protect individuals against panic attacks? As discussed in chapter 1, the exercise-affect relationship, crosssectional and longitudinal studies suggest a direct relationship between physical inactivity and anxiety severity/diagnosis. In fact, recent findings suggest that physical inactivity in patients with $P D$ is associated with more severe symptoms of panic. In chapter 7 , we report preliminary findings on the relationship between levels of physical activity and panic severity in a sample of patients with PD. We also sought to characterize the association between exercise activities with the different symptom dimensions of panic. With basis on our experimental data, we predicted that lower panic severity scores associated with exercise would be explained, at least in part, by the severity and frequency of panic attacks.

An important and continuing objective of our lab is to understand the biological underpinnings in experimental panic provocation. In addition to the studies on the antipanic effects of exercise, in chapter $\mathbf{8}$, we refined a proposed metabolic hypothesis where experimental panic attacks are explained as a primal defensive behavior linked to acute brain acidosis. In this review, the findings that link respiration with arousal and panic are integrated with the acid-base changes produced by techniques that are used to experimentally induce panic. The heuristic value and the potential experimental scenarios that can test this model are also discussed. 


\title{
Chapter 1:
}

\section{Part 1. Experimental models of panic}

\author{
Esquivel G, Schruers K and Griez E
}

Academic Anxiety Center and School for Mental Health and Neurosciences,

Faculty of Medicine, Health and Life Sciences, Maastricht University, Maastricht, The Netherlands

Published in part as: Esquivel, G., K. Schruers and E. Griez (2008). Experimental models: panic and fear. Handbook of Anxiety and Fear. R. J. Blanchard, D. C. Blanchard, G. Griebel and D. J. Nutt. Amsterdam, Elsevier B.V. 17: 413-435 
Introduction: Experimental models of panic

\section{Summary}

The main paradigm of experimental psychiatry is the carefully controlled, time limited production of a pathological picture in volunteering subjects, under safe laboratory conditions. Most often, such a procedure relies on either the manipulation of a physiological parameter or administration of a pharmacological agent, in order to unveil underlying vulnerabilities, believed to be directly related to the pathogenesis of the disorder. Because they intentionally put the organism under strain, such procedures have been referred to as "challenges". The interest of an experimental model mimicking a clinical condition lies in its validity. Insights in the underlying pathophysiology will progress to the extent that a particular challenge can be linked to a syndrome in particular individuals, who are also prone to the development of the same syndrome in real life environment. In other words, experimental models are supposed to act as biological markers of clinical disorders. Human models of panic and fear are scrutinized under different aspects for their validation. These aspects include safety, convergence, discrimination, reliability and clinical validity. However, these aspects of validation can become blur without a clear agreement on what is called anxiety, fear or panic, and their correspondence with spontaneous panic attack and situational panic attacks. In this scope, animal models have that have mapped fear and anxiety in two different defense systems may contribute to clarify this issue. Ultimately, the identification of a reliable biomarker can pave the way for studies into the genetic determinants of susceptibility to fear and panic and new therapeutic approaches. 


\section{Chapter 1}

\section{Introduction}

The experimental method is a procedure designed to falsify a prediction that was constructed by means of inferences from observed phenomena in nature (Verburg, Perna et al. 2001). This procedure, which involves the manipulation of models of such phenomena in a laboratory, aids in the construction of knowledge that would be otherwise very difficult to access by other means. In this way, human and animal models of psychopathology complement each other in the sense that the former have a clear face validity, whereas in animals, these models can delineate the neurochemical and neuroanatomical underpinnings of the mental workings in a much more powerful way. Conceiving experimental models of fear and panic in humans is an exercise of making inferences that claim a correspondence between human linguistic labels of brain processes (LeDoux 1996) and the observable defensive behavior of other mammals. Nevertheless, the need for human models of even one human anxiety disorder -panic- to be constantly reshaped by the overwhelming advances in the neurobiological understanding of defensive behavior in animals (and visa versa) cannot be understated (Gorman, Kent et al. 2000). In a broad sense, the ultimate goal of experimental models of panic, anxiety and fear is to achieve valid clinical diagnosis, currently based on epidemiological data (e.g. DSM-IV) and the development of means to effectively treat patients with anxiety disorders. Because these models intentionally put the organism under strain, such procedures have been referred to as "challenges". In fact, the principle of theses challenges resembles some procedures used in clinical medicine, for instance the glucose tolerance test, or an effort ECG. In both cases the diagnostic procedure seeks to evidence an underlying vulnerability that may not be obvious during routine examination.

One straightforward approach to the human models of panic and fear is to look at their construct validity, In other words, investigate whether the observed pattern in a model corresponds to that of the real life psychopathological process. In the case of panic for example, some authors have proposed the basic operational criteria required for a model validation (Guttmacher, Murphy et al. 1983): safety, convergence, discrimination, reliability and clinical validity:

1. Safety. When dealing with models that can elicit defensive responses, safety is an essential component of any procedure performed in human subjects. The symptoms provoked by an experimental maneuver should be temporary, short lived, readily reversible and hold no foreseeable health risks.

2. Convergence. With regard to convergence, the experiences produced by the model should mimic naturally occurring cognitive and neurovegetative symptoms of fear and panic. Additionally, experiences should be idiosyncratic to each individual subject. 
3. Discrimination. A feature that has received particular attention is discrimination (specificity) or the capability of the model to identify those with the affected condition from those without it. Although it might seem that absolute specificity is desirable, arguably, a relative specificity may actually better reflect the psychopathological processes involved in panic disorder (PD). This notion stems from research that regards defensive behaviors as a neurobiological evolutionary derived function of the brain present in all mammals (McNaughton and Corr 2004) and as well as studies that suggest that vulnerability for PD, for instance, may obey homeostatic processes that follow a continuous distribution with those affected individuals in one extreme (Battaglia and Perna 1995; Bellodi, Perna et al. 1998; Swain, Koszycki et al. 2003).

4. Reliability. Or re-test reliability refers to the reproducibility of the effects of the model after consecutive trials.

5. Clinical validation. Clinical validation relates to one of the most powerful and useful elements of construct validity. For example, medication that is effective for PD and reduces the effects of a panic-inducing experimental manipulation, can be linked to a large amount of pharmacological studies performed in animal models (Blanchard, Griebel et al. 2003). Clinical validation should also include effective non-pharmacological interventions (i.e. cognitive-behavioral therapy) that effectively reduce the symptoms produced by a model.

It is clear that an agreement on what we call anxiety, fear or panic is essential when describing experimental models for them. Unfortunately, a uniform definition does not exist to date. Hence, an important limitation in the validation of models for anxiety disorders is the controversy concerning the meaning of the concept of panic (or fear). Some suggest that it is an intense form of general anxiety, as opposed to those stressing that it is an evolutionary more primitive emotion, activated by physiological disturbances that warn the organism of impending danger (Andreatini, Blanchard et al. 2001). Allegations in favor of the idea of panic as an intense form of general anxiety (i.e. the idea that anxiety, fear and panic are on a continuum) stem from the fact that anti-panic medication, such as antidepressants and benzodiazepines, can also provide relief for phobias and generalized anxiety. Models that support this view have linked situational panic and laboratory induced panic attacks, with neuroendocrine and physiological responses that activate defensive networks that are probably integrated in the amygdala (Charney 2003), and provide a framework that may also explain phobic avoidance and agoraphobia (Gorman 2003).

On a different view, others have put forward that the phenomenon of spontaneous panic is clearly distinct from that of situational panic and other anxiety symptoms related to $P D$. They base their claim on animal research that has arranged defensive behavior in a two dimensional hierarchically organized brain system (McNaughton and Corr 2004). This concept integrates concepts of defensive distance and defensive direction (Blanchard, Griebel et al. 
Chapter 1

2003). Defensive approach (i.e. anxiety) is favored when the distance from the source of danger allows the possibility for elaborate and complex behaviors, whereas defensive avoidance (i.e fear and panic) will be predominant when it is necessary to deal with nose-tonose danger (Graeff 2004). In this view, (generalized) anxiety is (i) linked to responses that deal with the conflicts of approach to potential danger (distant or imagined), (ii) suppressed by anxiolytic drugs, (iii) integrated in the amygdala and other forebrain structures, and (iv) activates the HPA axis. Fear (and spontaneous panic as its most primitive and intense form), on the contrary, is (i) related to behavior adequate to escape imminent danger (i.e. suffocation), (ii) reduced by SSRI's, and (iii) organized in more primitive structures that do not activate the HPA system (Graeff 2004; Graeff, Garcia-Leal et al. 2005). Some support in favor of this notion is provided by studies showing that when spontaneous panic is distinguished from situational panic and anticipatory anxiety, the crucial role of respiratory symptoms (Briggs, Stretch et al. 1993; Uhlenhuth, Matuzas et al. 2000) and lack of HPA axis activation (Graeff, Garcia-Leal et al. 2005) becomes evident. Other studies have also shown that symptoms of human pathological fear are not related to HPA axis activation (Potts, Davidson et al. 1991; Uhde, Tancer et al. 1994; Martel, Hayward et al. 1999).

This chapter will attempt to describe the most prominent and current experimental maneuvers that claim to model human panic. After a general description of the conception, the technique, and the possible mechanisms, the studies that validate the model according to the described basic criteria will follow. Finally, without trying to solve the anxiety-panic debate, studies that can potentially address this issue will be considered and incorporated throughout the description of each model.

Sodium Lactate and other hyperosmotic infusion techniques

\section{Background}

Lactate the dissociated cationic form of lactic acid in biological fluids, is a ubiquitous substance, constantly produced as result of mammalian anaerobic metabolism. Lactate has been historically demonized as a wastage product responsible for pain and exhaustion during exercise (Leverve and Mustafa 2002). Nevertheless, is actually actively recycled during physical exertion and may play an important role in energetic homeostasis in both physiological and extreme conditions. Moreover, lactate may be the most important source of energy for neurons where it is glycolytically converted by astrocytes from glucose (Tsacopoulos and Magistretti 1996).

Lactate occurs in two stereoisometric forms, L-lactate and D-lactate, where the L-lactate is an abundant intermediate of mammalian metabolism, and D-lactate is usually of 
Introduction: Experimental models of panic

bacterial origin. Human metabolism of D-lactate is known to occur, but is only in a very small proportion of that of L-lactate (Kondoh, Kawase et al. 1992).

The standardized use of lactate infusions as a panicogenic maneuver was established by Pitts and McClure (1967) initially to provoke anxiety in patients with anxiety neurosis (then, the term panic was not extensively used). Their idea stemmed from the previous work of Cohen and White (1951) in patients with neurocirculatory asthenia (a diagnostic entity that overlaps with PD) that showed abnormally high levels of blood lactate and a low oxygen uptake in response to physical exercise. The lactate infusion can probably still be regarded as the most extensively used panicogenic maneuver. The use of bicarbonate and hypertonic infusions, despite providing with interesting findings, is scarce.

\section{Features \& technique}

As a model of panic provocation, sodium lactate (typically racemic) is intravenously administered in a $0.5 \mathrm{~mol} / \mathrm{L}$ concentration dose of $10 \mathrm{~mL} / \mathrm{kg}$ over 20 minutes. Pure $\mathrm{D}$ - or L-lactate infusions in panic patients have been performed. However, the results of these studies are not clear (Gorman, Goetz et al. 1990; Klein 1993). A larger concentration of 1.0 $\mathrm{mol} / \mathrm{L}$ has been used in a small sample of PD patients, nevertheless with inconclusive results (Ehlers, Margraf et al. 1986; Klein and Ross 1986). Sodium bicarbonate and hypertonic saline have been administered in a similar fashion as a $2.5 \mathrm{~mL} / \mathrm{kg}$ of $8.4 \%$ and $10 \mathrm{~mL} / \mathrm{kg}$ of $3 \%$, respectively, with a point-to-panic usually reached after about 10 minutes (Liebowitz, Gorman et al. 1985; Gorman, Battista et al. 1989; Peskind, Jensen et al. 1998).

Lactate infusions produce various physiological changes in all subjects such as paradoxical hyperventilation, an increase in heart rate and in blood pressure (Liebowitz, Gorman et al. 1985; Peskind, Jensen et al. 1998). Because the effects of lactate on cell metabolism there is a parallel increase of bicarbonate with blood lactate levels. In other words, the metabolism of lactate in the tricarboxylic-acid cycle leads to the cytosol consumption of a proton ion and therefore the generation of a bicarbonate ion (Zhou 2005). Sodium bicarbonate infusions in PD patients also produce paradoxical hyperventilation (Gorman, Battista et al. 1989). More recent research on the effects of a sodium bicarbonate infusion on brain intracellular $\mathrm{pH}$ (Nakashima, Yamashita et al. 1996) shows that the maneuver produces a significant shift towards acidity that is likely to explain the hyperventilation seen with lactate and bicarbonate panic provocation challenges. Interestingly, a panicogenic hypertonic saline infusion does not provoke hyperventilation (Peskind, Jensen et al. 1998). Increased heart rate and blood pressure is not surprising when the amount of hyperosmotic fluids administered that initially stimulate vasopressin and the locus ceruleus are considered: i.e. an infusion with lactate, bicarbonate or hypertonic saline (King and Baertschi 1992; Ganong 1997). Other studies from the Bristol group suggest that lactate produces shifts in CNS brain electrolytes 
and glucose may provoke changes in noradrenergic and GABA tone in relevant brain structures (George, Glue et al. 1990; George, Lindquist et al. 1995). Additionally, some of the physiological changes during a lactate infusion can be attributed to a reduction in parasympathetic activity (George, Nutt et al. 1989)

The idea that panic attacks are linked with HPA-axis activation remains still controversial (Graeff, Garcia-Leal et al. 2005). Extensive work concludes that lactate-induced panic is not linked to HPA-axis activation (Hollander, Liebowitz et al. 1989; Coplan, Goetz et al. 1998) and at least one study suggests that neither is hypertonic saline-induced panic (Peskind, Jensen et al. 1998). It has been speculated that the rise in atrial natriuretic peptide during a lactate infusion may blunt the cortisol response (Kellner, Herzog et al. 1995). However, in another study pre-medication with atrial natriuretic peptide did not affect the characteristic HPA activation of the putative panicogen CCK (Wiedemann, Jahn et al. 2001).

\section{Underlying mechanisms}

The mechanism behind lactate-induced panic remains obscure. Early studies have proposed and discarded the implication of peripheral and central adrenergic surge, ionized calcium or metabolic alkalosis (Liebowitz, Gorman et al. 1986).

Because a noradrenergic disturbance has been postulated as an underlying mechanism in panic (Charney and Heninger 1986), lactate infusions have been performed after premedication with the $\beta$-adrenergic blocker propranolol (Gorman, Levy et al. 1983) and the $\alpha$-adrenergic agonist clonidine (Coplan, Liebowitz et al. 1992). Propranolol did not have any effect on lactate panic-provocation, whereas clonidine had a partial panic-reducing effect. Clonidine, however, has shown to have a therapeutic effect (Valenca, Nardi et al. 2004) and is associated with a release of atrial natriuretic peptide (Mukaddam-Daher, Lambert et al. 1997).

The fact that most of the racemic lactate (L-lactate) used in infusions is metabolized to pyruvate, with a consecutive increase in bicarbonate, has lead to the idea that the infusion could ultimately result in an increase in partial-pressure of $\mathrm{CO} 2$ (pCO2) by back-titration (i.e. $\mathrm{HCO}-$ and $\mathrm{H}+\rightarrow \mathrm{CO} 2$ and $\mathrm{H} 2 \mathrm{O}$ ). This metabolic hypothesis, that unified the mechanisms behind lactate and $\mathrm{CO} 2$ induced panic, was tested in a protocol using bicarbonate (Gorman, Battista et al. 1989). Results from this study, however, suggest at best a contribution of lactate "metabolism" to CO2 in lactate's panicogenic effect. The fact that D-Lactate has shown to also be panicogenic (Gorman, Goetz et al. 1990), thought not to have any metabolism to pyruvate, would exclude the possible contribution of $\mathrm{CO} 2$. However, the large increases of bicarbonate seen in this trial cannot rule this out. A spectroscopy study indicating brain intracellular acidification after an infusion with bicarbonate (Nakashima, Yamashita et al. 1996) also lends some empirical support to the metabolic hypothesis of lactate-induced panic. 
Introduction: Experimental models of panic

In the 1980's, Carr and Sheehan (1984) proposed a model where a lactate infusion and its metabolism to pyruvate would reduce NAD+ to NADH and $\mathrm{H}+$ with a consecutive drop in neuronal $\mathrm{pH}$, depending on its permeability through the blood-brain barrier. Although lactate probably does not cross the blood-brain barrier, work performed with proton-NMR spectroscopy to measure the brain lactate response during a racemic lactate infusion shows a larger diffuse brain lactate increase in PD patients (Dager, Friedman et al. 1999). Additionally, acute and disproportionate brain lactate elevations are seen in PD after voluntary hyperventilation $(\mathrm{VH}$ ) as measured with $1 \mathrm{H}-\mathrm{NMR}$ techniques (Dager, Strauss et al. 1995). It is plausible that this metabolic scenario can lead to brain intracellular acidification (van Rijen, Luyten et al. 1989; Maddock 2001), potentially stimulating $\mathrm{H}_{+} / \mathrm{CO} 2$ chemoreceptors in the same fashion as $\mathrm{CO} 2$ inhalations.

As mentioned, a lactate infusion (and hypertonic saline) constitutes a high volume hyperosmotic fluid load that provokes an increase in blood pressure, heart rate and hemodilution. This brings about the possible role of the so called osmosensitive circumventricular organs (Ganong 1997). These areas, with fenestrated capillaries at many sites that have direct link to the hypothalamus and other structures involved in panic, are candidate sites for the action of hyperosmotic challenges (Shekhar and Keim 1997). In an animal model with a disruption of GABA-mediated inhibition in the dorsal medial hypothalamus, the panic-like effect of lactate was mediated by angiotensin II (Shekhar, Johnson et al. 2006). Shifts in neuroactive steroids that modulate GABA activity seem to be associated with lactate induced panic in PD patients (Strohle, Romeo et al. 2003) and are in line with a possible role of the circumventricular organs. It is attractive to speculate that the dorsal medial hypothalamus is involved in lactate induced panic since it is considered a key anatomical structure in animal models of panic attacks and defensive behavior, together with the periaqueductal grey (PAG) and the amygdala (Schenberg, Bittencourt et al, 2001; Canteras 2002).

Validity

In those patients that respond to a lactate infusion there is a considerable symptom convergence with their reported natural spontaneous panic attacks (Liebowitz, Fyer et al. 1984; Dillon, Gorman et al. 1987; Goetz, Klein et al. 1994), except maybe for tingling which appears to be a specific hypocalcemic effect of the infusion. When a lactate infusion is compared to bicarbonate and hypertonic saline, the lactate specific tingling seems to be absent only in the former (Gorman, Battista et al. 1989). However, a fundamental absence of the same degree of dyspnea is absent in the latter (Peskind, Jensen et al. 1998). Some studies place emphasis on the lack of specificity for dyspnea in panic attacks (Vickers and McNally 2005) However, this issue is clarified when a distinction is made between 
Chapter 1

spontaneous panics and anticipation/situational anxiety attacks (Briggs, Stretch et al. 1993) which are considered to have phenomenological overlap but a distinct neurobiology (Andreatini, Blanchard et al. 2001; Uhlenhuth, Leon et al. 2006).

A large body of evidence developed by the group from Columbia University (Gorman, Papp et al. 1990) has shown that PD patients are more vulnerable to a lactate infusion than healthy subjects, patients with depression, generalized anxiety disorder (GAD), obsessive compulsive disorder, social phobia (SP) or bulimia (Liebowitz, Fyer et al. 1984). Moreover, lactate seems to be more specific to a history of panic attacks than the clinical diagnosis of PD (Cowley, Dager et al. 1986; Buller, von Bardeleben et al. 1989). The specificity of lactate in PD, however, is not without controversy as patients with GAD (Cowley, Dager et al. 1988) and premenstrual dysphoric disorder (Facchinetti, Romano et al. 1992) have a larger reaction than healthy controls, but smaller when compared to PD patients. Also, lactate infusions may provoke flashbacks in patients with posttraumatic stress disorder (PTSD) (Jensen, Keller et al. 1997). Panic rates for a classic racemic sodium lactate infusion vary, but a sample from two large studies report panic attacks in over $65 \%$ of PD patients and $0 \%$ of healthy controls (Liebowitz, Fyer et al. 1984; Gorman, Battista et al. 1989).

A well designed study to evaluate the reliability of lactate and isoproterenol-induced panic attacks, reveals that these maneuvers do not display any sensitizing or desensitizing effect on PD patients after three consecutive trials (Yeragani, Balon et al. 1988). Many pharmacological substances and interventions that are therapeutic in PD have been used to block the effects of a lactate infusion. High potency benzodiazepines (e.g. Alprazolam) can cancel the panicogenic effects of lactate (Liebowitz, Fyer et al. 1986; Cowley, Dager et al. 1991) as opposed to diazepam which has shown to have no effect (Liebowitz, Coplan et al. 1995). Antidepressants such as tricyclics (Yeragani, Pohl et al. 1988) and SSRI's (Dager, Richards et al. 1997) when given for several weeks have also shown to reduce the effects of lactate on measures of panic.

\section{Carbon Dioxide}

\section{Background}

Carbon dioxide, as lactate an end-product of carbohydrate metabolism, is constantly produced in the cell yet it rarely accumulates as it is readily processed by the bicarbonate system and transported from tissues to the lungs where it is finally excreted (Ganong 1997).

Although the experimental panicogenic properties of $\mathrm{CO} 2$ have been described since 1951 (Cohen and White), it was not until the 1980's when Marcel Van den Hout and Eric Griez (1984) at Maastricht University applied inhalations with $35 \% \mathrm{CO} 2$, that were considered "anxiolytic" at the time, and found that PD patients experienced brief periods of intense 
cognitive and physical symptoms that resemble spontaneous panic attacks. About the same time, Jack Gorman (1984) at the University of Columbia in New York used a continuous inhalation of $5 \% \mathrm{CO} 2$ during $\mathrm{VH}$ as way of controlling hypocapnia, and unexpectedly found that using $\mathrm{CO} 2$ was more panicogenic than the hyperventilation. Carbon dioxide challenges are currently one of the most extensively used models for panic.

\section{Features \& technique}

The two main methods of delivering $\mathrm{CO} 2$ as an experimental panicogen are (i) a single- or a double-breath 35\% inhalation through a mask Nan den Hout and Griez 1984; Fyer, Uy et al. 1987) and (ii) the continuous 5-7\% inhalation in a canopy for 15-20 minutes (Gorman, Papp et al. 1994). A single-breath 35\%CO2 / 65\%O2 mixture inhalation of at least $80 \%$ of the subject's vital capacity and subsequent breath holding for 4 seconds, is widely used (Perna, Battaglia et al. 1994). Variations of the $35 \% \mathrm{CO} 2$ model include a double-breath hold with the mixture for 4-8 seconds (Nardi, Valenca et al. 2006), a continuous inhalation for 30 seconds (Papp, Klein et al. 1993) and 5 repeated vital-capacity inhalations (Schmidt, Trakowskl et al. 1997) which apparently increases sensitivity with a decrease in specificity. The $35 \%$ challenge elicits its panicogenic effects in a matter of seconds (Griez, van den Hout et al. 1987), whereas the inhalation of 5-7\% does the same after several minutes (Gorman, Papp et al. 1994). Other forms of administration include the Read re-breathing method with $7 \% \mathrm{CO} 2$ (Zandbergen, Pols et al. 1991), a continuous positive air pressure with 5.5\% (Sanderson, Rapee et al. 1989) and a 20-second inhalation of 20\% CO2 (Prenoveau, Forsyth et al. 2006). Simple breath-holding with room-air has been currently used as method to endogenously increase body $\mathrm{CO} 2$ levels where subjects are usually instructed to stop breathing following a normal exhalation and hold their breath for as long as possible (Nardi, Nascimento et al. 2003).

Because of the biological importance of its elimination, $\mathrm{CO} 2$ elicits a powerful ventilatory stimulation mediated by peripheral and central $\mathrm{CO} 2 / \mathrm{H}+$ chemoreceptors. In the presence of carbonic anhydrase, $\mathrm{CO} 2$ interacts with water to form carbonic acid, which is highly unstable and rapidly dissociates into $\mathrm{H}+$ and bicarbonate. The accumulation of $\mathrm{H}+$ without a compensatory increase of $\mathrm{HCO}$ - would lead to a decrease in $\mathrm{pH}$. The single inhalation of $35 \% \mathrm{CO} 2$ produces a rapid intravascular increase of $\mathrm{pCO} 2$ and a decrease of $\mathrm{pH}$ that lasts about 10 seconds and is immediately followed by a 2-minute hyperventilation-driven alkalosis (Griez, van den Hout et al. 1987). Notoriously, CO2 may also provoke a dramatic increase in cerebral blood flow (CBF) that is contingent on the perivascular levels of $\mathrm{CO} / \mathrm{H}+$ (Pandit, Mohan et al. 2003). Studies that have assessed the relationship between CBF and anxiety/panic during $\mathrm{CO} 2$ provocation are not clear-cut. The $5 \%$ challenge has shown that higher subjective anxiety in a mixed GAD-healthy subject group is related to a lesser increase in CBF (Mathew and Wilson 1988; Mathew, Wilson et al. 1997a) as measured with the xenon- 


\section{Chapter 1}

133 inhalation technique. On the other hand, more recent work with transcranial doppler and the $35 \% \mathrm{CO} 2$ probe in PD patients shows that they display a larger increase in basilar artery blood flow velocity than healthy controls after the challenge.

Neuroendocrinological studies in $\mathrm{CO} 2$ induced panic have revealed that the challenge can elicit a small HPA activation in healthy volunteers and PD patients (van Duinen, Schruers et al. 2005; van Duinen, Schruers et al. 2006). This activation however is not related to any subjective measures of panic nor does it discriminate panic patients from healthy controls. On the other hand, the Bristol group has found substantial cortisol and ACTH activations after a challenge with 35\% CO2 in healthy volunteers (Argyropoulos, Bailey et al. 2002; Kaye, Buchanan et al. 2004). Remarkably, the latter study shows a moderate positive correlation between peak anxiety and plasma cortisol change.

\section{Underlying Mechanisms}

The relatively recent discovery of acid-sensing ion channels involved in fear conditioning (Wemmie, Askwith et al. 2003; Wemmie, Coryell et al. 2004) and the pH chemosensitivity of midbrain 5-HT neurons (Severson, Wang et al. 2003; Richerson 2004) makes it mechanistically tentative to view the response to the $\mathrm{CO} 2$ inhalation as an affective expression related to brain acidosis.

In an effort to determine if central acidification was relevant to the subjective response to $\mathrm{CO} 2$ induced symptoms in $\mathrm{PD}$, two groups of researchers tested the subjective effects of $1 \mathrm{gram}$ of intravenous acetazolamide (Mathew, Wilson et al. 1989; Gorman, Papp et al. 1993). Results from these studies are consistent with the fact that the maneuver does not provoke panic, and moreover, one group reported a reduction in the levels of anxiety with the medication (Gorman, Papp et al. 1993). It is remarkable that acetazolamide has shown to increase levels of $\mathrm{CO} 2$ and decrease $\mathrm{pH}$ in the extracellular compartments of the brain, yet without an effect on cerebral intercellular pH (Bickler, Litt et al. 1988; Vorstrup, Jensen et al. 1989). This finding may explain the drug's lack of an acute effect on ventilation (Mathew, Wilson et al. 1989; Gorman, Papp et al. 1993) as minute ventilation in human healthy volunteers has an inverse relationship with brain intracellular $\mathrm{pH}$ (Jensen, Thomsen et al. 1988). One study specifically designed to determine if the response to CO2 is dosedependent, has shown that the panic reaction to a CO2 challenge is related to the amount of CO2 administered (Griez, Colasanti et al. 2007). In general, in studies use a double inhalation vs. a single inhalation or a continuous inhalation of $7 \%$ vs. $5 \%$, the larger dose of CO2 is invariably related to a higher panic rate in PD patients (Rassovsky and Kushner 2003; Nardi, Valenca et al. 2006).

Recently, Bailey and coworkers (2003) has set forward a hypothesis where $\mathrm{CO} 2$-induced panic initiates in the brainstem chemosensory noradrenergic neurons including 
Introduction: Experimental models of panic

the locus ceruleus with a subsequent involvement of the central nucleus of the amygdala and the hypothalamus.

Neuroimaging studies have revealed that $\mathrm{CO} 2$ inhalations and $\mathrm{CO} 2$ induced breathlessness are related to activations in several brains structures that are thought to be implicated in affective responses including the midbrain, hypothalamus, hippocampus, cingulate gyrus and cerebellum (Corfield, Fink et al. 1995; Liotti, Brannan et al. 2001). Interestingly, activation of the cerebellum during CO2 elicited breathlessness has been hypothesized to have a role beyond the traditional implication in motor activity to include primal emotions that would serve ecological purposes (i.e. escaping suffocation) (Parsons, Egan et al. 2001).

Validity

A diversity of studies confirms that the symptoms elicited by $\mathrm{CO} 2$ inhalations are similar in quality, duration and severity to panic attacks that occur naturally (Griez, Lousberg et al. 1987; Perna, Battaglia et al. 1994). The study by Perna et al. (1994a) additionally concludes that the risk of having a panic attack is significantly more reliably predicted by reactivity to $\mathrm{CO} 2$ than by meeting diagnostic criteria for PD. In healthy volunteers, a positive response to a $20 \% \mathrm{CO} 2$ challenge predicted the future onset of spontaneous panic attacks in a 2-year prospective follow-up study (Schmidt, Maner et al. 2007). Taken together, these data are consistent with a psychological model of PD where a spontaneous panic attack is a core psychopathological element that, when present in individuals with an anxious cognitive style, can precipitate the dysfunctional set of behaviors and cognitions (Bouton, Mineka et al. 2001) characteristic of PD.

A single 35\% CO2 inhalation after 3 consecutive challenges at a weekly interval has displayed good reliability (Perna, Battaglia et al. 1994; Verburg, Pols et al. 1998), although there appears to be some desensitization after a prolonged series of challenges (van den Hout, van der Molen et al. 1987).

Carbon dioxide can elicit panic in $20-90 \%$ of patients with PD and 0 to $38 \%$ of healthy controls, depending on the gas concentration, method of delivery and operational definition of a panic attack (Papp, Martinez et al. 1997; Rassovsky and Kushner 2003). The CO2 challenge specifically elicits a panic reaction in PD patients as opposed to healthy subjects (Griez, Lousberg et al. 1987). Similar results are obtained when PD patients are compared to patients with obsessive-compulsive disorder (OCD) (Griez, de Loof et al. 1990) and subjects with other anxiety and mood disorders (Griez 2001). Recent research suggest that a continuous inhalation of $7.5 \% \mathrm{CO} 2$ may be used as a model for $\mathrm{GAD}$, based on the differential modification of its effects by lorazepam and paroxetine in healthy volunteers (Bailey, Kendrick et al. 2007). Nevertheless, patients with GAD have a smaller panic and anxiety 
response to $5 \%$ and $35 \%$ CO2 compared with PD patients (Holt and Andrews 1989; Verburg, Griez et al. 1995; Perna, Bussi et al. 1999). Additionally, GAD patients show an anxiety response similar to that of healthy controls using the 5\% CO2 inhalation (Mathew and Wilson 1988; Mathew, Wilson et al. 1997). The Bristol group has shown, in separate studies, that healthy volunteers and GAD patients display increased anxious to the continuous inhalation of 7.5\% CO2 (Seddon, Potokar et al.; Bailey, Argyropoulos et al. 2005).

Several studies confirm that effective anti-panic medications, such as MAOl's (Perna, Cocchi et al. 1994), SSRI's (Pols, Hauzer et al. 1996) and high potency benzodiazepines (Pols, Verburg et al. 1996), are capable of significantly reducing the reaction to a CO2 challenge in PD patients. Moreover, some of these medications have shown to be capable of blocking a $\mathrm{CO} 2$ challenge after only a week of treatment and before a full therapeutic response was obtained (Perna, Bertani et al. 1997).

\section{Cholecystokinin}

\section{Background}

In the early seventies, a small peptide with gastrin-like immunoreactivity was discovered in the mammalian brain Nanderhaeghen, Signeau et al. 1975). Despite a quite heterogeneous distribution, this substance, which was identified as the sulphated C-terminal octapeptide of the gut hormone CCK, proved to be the most abundant neuropeptide in the human brain. The octapeptide CCK-8S and, more important, corresponding receptors, were found mainly in the cortex, caudate nucleus, putamen and hippocampus. Additionally, somewhat lower levels were found in the septum PAG and other limbic structures. It was later discovered that the tetrapeptide CCK-4, consisting of the very first CCK-8S amino acids, was able to activate brain receptors (CCK-B receptors). In the late eighties, an investigator noticed a brief, but strong feeling of anxiety after self-administration of CCK. This led to an open label trial in a group of 10 healthy volunteers, confirming that intravenous injections of CCK, in various dosages between 20 and $100 \mu \mathrm{g}$, had definite anxiogenic properties (de Montigny 1989). In the mid nineties, investigators began to use pentagastrin, an easily available agonist of CCK receptors, as an alternative to CCK-4 for the experimental study of panic (Abelson and Nesse 1994). Pentagastrin, a synthetic analogue of gastrin, was developed for the diagnostic evaluation of gastric acid secretory function. It is a 5-amino acid peptide, which with its 4 amino acids C-terminal sequence is identical to CCK-4.

\section{Features \& technique}

CCK-4 and pentagastrin are administered as a bolus intravenous infusion. CCK-4 is usually administered in a 1 to $3 \mathrm{~mL}$ saline solution with dosages of $25 \mu \mathrm{g}$ in PD patients and $50 \mu \mathrm{g}$ in 
Introduction: Experimental models of panic

healthy volunteers. Pentagastrin is generally administered in a less than $1 \mathrm{~mL}$ of saline solution with dosages from 0.5 to $0.6 \mu \mathrm{g}$ per $\mathrm{kg}$ in both $P D$ and healthy volunteers. The maneuver elicits its characteristic behavioral effects about 20 seconds after the infusion with a mean duration of about 20 minutes (Bradwejn, Koszycki et al. 1990).

CCK-B receptor agonist administration is accompanied by noticeable cardiorespiratory alterations. Well-controlled studies have shown a robust increase of heart rate, blood pressure and minute ventilation only a few minutes after administration (Abelson and Nesse 1994; Bradwein, LeGrand et al. 1998; Koszycki, Zacharko et al. 1998). Research into the relationship between objective cardiorespiratory measures and subjective panic measures have shown that these are rather asynchronous. Although one study did find a relationship between diastolic blood pressure and a positive panic response to CCK-4, these findings have not been replicated in recent, carefully designed studies (Eser, Schule et al. 2007). Pentagastrin and CCK-4 also elicit a considerable activation of the HPA axis. Abelson and coworkers (1994) analyzed ACTH, cortisol and growth hormone responses to the pentagastrin challenge. Pentagastrin produced a consistent and potent activation of the HPA axis, although interpretation of the authors was that HPA activation was not related to the anxiogenic response. Furthermore, a recent randomized, placebo-controlled study in 85 healthy volunteers using CCK-4 found no relationship between HPA activation and measures of panic response (Eser, Schule et al. 2007).

\section{Underlying Mechanisms}

Several neurotransmitter systems have been postulated to mediate the effects of CCK-4 and pentagastrin. The actions of CCK-B receptor agonists elicited panic symptoms can be almost entirely blocked by the CCK-B receptor antagonist L-365,260 in both healthy volunteers (Lines, Challenor et al. 1995) and PD patients (Bradwein, Koszycki et al. 1994). Cl-988, another CCK-B receptor antagonist, also showed to reduce the effects of CCK when administered to healthy volunteers (Bradwejn, Koszycki et al. 1995). However, its effects were modest which is probably due to a poor systemic availability. These finding altogether suggest a mediation of CCK-B receptors in the panicogenic effects of CCK-4 and pentagastrin in humans. This, in turn, has lead researchers to hypothesize that CCK receptors might play a major role in the underlying mechanisms of spontaneous panic attacks and PD. Unfortunately, well controlled studies using CCK antagonists have failed to show any therapeutic effects in PD patients (Kramer, Cutler et al. 1995; Pande, Greiner et al. 1999). While some research suggests that the serotonergic, the noradrenergic and the benzodiazepine receptor systems may modulate the panicogenic effects of CCK, their mediation is generally not supported (Bradwejn, Koszycki et al. 1994; McCann, Morgan et al. 1997; Khan, Liberzon et al. 2004; Maron, Toru et al. 2004; Toru, Shlik et al. 2006). 
Based on findings from animal research, it is attractive to speculate that HPA-axis activation plays an important role in the panicogenic effects induced by CCK-B agonists (Strohle, Holsboer et al. 2000). As mentioned previously, CCK-4 and pentagastrin elicit a clear HPA-axis activation that is rather asynchronous with the provoked panic arousal (Graeff, Garcia-Leal et al. 2005). In this scope, a recent study has shown that premedication with a mineralocorticoid antagonist before a CCK challenge in healthy men increased baseline cortisol. However, no effect was found on baseline ACTH, or either CCK-stimulated cortisol or ACTH (Otte, Moritz et al. 2007). In a similar protocol, premedication with the synthetic progestational hormone megestrol blunted the HPA-axis activation typically seen with a CCK (Raedler, Jahn et al. 2006). Thus, despite the modifications produced by the mineralocorticoid antagonist and megestrol on ACTH and cortisol activation, no effects were seen on the panicogenic effects of a CCK challenge. On a different perspective, some studies have explored the relationship between the CCK challenge and the activity of neuroactive steroids that can allosterically modulate neurotransmitter receptors. The CCK elicited panic symptoms in $P D$ patients has been correlated with changes in metabolites of progesterone that modulate the activity of GABA-A receptors and are consistent decrease central GABA-ergic tone (Strohle, Romeo et al. 2003).

Neuroanatomical studies in both animal and human have yielded compelling results. Research has shown that the dorsal PAG CCK-2 (CCK-B) receptors modulate anxious behavour displayed by rodents in an elevated plus-maze model (Netto and Guimaraes 2004) and freezing and escape behavior evoked by electrical stimulation (Bertoglio, de Bortoli et al. 2007). The former study also showed that the administration of CCK-B agonists in the dorsal $P A G$ induced Fos expression in other brain regions related to defensive behaviour such as the raphe nuclei, medial amygdala, medial hypothalamus and paraventricular hypothalamus. As mentioned earlier, the PAG, together with the medial hypothalamus and the amygdala, are key anatomical structures in animal models of panic attacks and defensive behavior (Schenberg, Bittencourt et al. 2001; Canteras 2002). A positron emission tomography (PET) study in healthy male volunteers was conducted to determine the changes in regional CBF in two time points after a CCK challenge (Javanmard, Shlik et al. 1999). This study revealed an early involvement of the hypothalamus and a later asymmetrical activation of the claustrum-insular that positively correlated with increments in the measurements of panic. A more recent study, using functional magnetic resonance imaging recordings in healthy volunteers exposed to 50 $\mu \mathrm{g}$ of CCK-4, has found a blood oxygen level-dependent (BOLD) activation in the insular cortices, temporal poles, cingulate gyrus, thalamic region and cerebellar vermis. These BOLD activations were more extensive and significant in those with a strong panic reaction to the challenge. Although the latter study does not report on any activation of the hypothalamus, as set forward by Javanmard and coworkers (Javanmard, Shlik et al. 1999), it is appealing to 
Introduction: Experimental models of panic

speculate an initial activation of the hypothalamus because of its interconnections with circumventricular organs including the area postrema, subfornical organ, and organum vasculosus lamina terminalis (Shekhar and Keim 1997). Intravenous infusions with CCK-B agonists may exert their effect by leaking through circumventricular organs, such as the area postrema (Olson, Freilino et al. 1993).

Validity

The technique of using CCK-B agonists as a safe method has been well established with hundreds of subjects. The symptoms induced by CCK-B agonists in PD patients seem to resemble their spontaneous panic attacks (Bradwejn, Koszycki et al. 1990; Abelson and Nesse 1994). At least one study attests the reproducibility of panic symptoms using an infusion with CCK-B receptor agonist in PD patients (Bradwejn, Koszycki et al. 1992).

In randomized placebo-controlled studies the effects of CCK-B agonists appear to be dose dependent, with higher dosages increasing the number of subjects reporting panic sensations (Bradwejn, Koszycki et al. 1991; Bradwejn, Koszycki et al. 1992; van Megen, Westenberg et al. 1994). However, at a given dosage, there is a significant difference in sensitivity to CCK between normal controls and patients with $P D$, the latter group appearing definitively more vulnerable to the pharmacological effect of CCK agonists. A series of wellcontrolled studies have compared the CCK vulnerability in other anxiety disorders. A pentagastrin challenge in a small sample of OCD patients suggests that these are more prone to respond with a panic attack than healthy controls (de Leeuw, Den Boer et al. 1996). Additionally, OCD symptomatology was not affected. One study compared the responses of patients with $P D$, patients with SP and healthy volunteers to pentagastrin (McCann, Slate et al. 1997). Both groups of PD and social phobics panicked in the pentagastrin condition. And although patients with $\mathrm{PD}$ had a slightly higher rate of formally defined panic attacks, 64\% versus $47 \%$, the difference was not significant. The rate of panic in the healthy control group was $11 \%$. This, together with a similar study (van Vliet, Westenberg et al. 1997), strongly suggests that patients with $\mathrm{SP}$ and $\mathrm{PD}$ have a similarly high sensitivity to CCK agonists. Nevertheless, Katzman (2004) and coworkers did not replicate these findings when they compared the vulnerability of OCD patients, SP patients and healthy volunteers to $20 \mu \mathrm{g}$ of CCK-4. In this study, the small sample size may have precluded the finding of a significant difference between the 50\% panic attack rates in social phobics and OCD and the $8.3 \%$ panic attack rate in healthy volunteers. One study addressed the issue of CCK sensitivity in GAD (Brawman-Mintzer, Lydiard et al. 1997). A limited sample of 7 patients and 7 age-matched healthy controls received a bolus infusion with $0.6 \mu \mathrm{g}$ per $\mathrm{kg}$ of pentagastrin on one occasion, and saline placebo on another occasion, according to a single blind design. Results from this study suggest that GAD patients are highly vulnerable to a pentagastrin challenge. Overall, 
Chapter 1

although the studies are relatively sparse and the samples quite small, most investigations reviewed above in other anxiety disorders with CCK analogues indicate that the high CCK sensitivity observed in PD may extend across the main other anxiety disorders.

Several investigations have shown that antipanic interventions can also reduce the effects of a challenge with CCK-B agonists. For instance, PD patients that respond to imipramine (Bradwejn and Koszycki 1994), fluvoxamine (van Megen, Westenberg et al. 1997) and citalopram (Shlik, Aluoja et al. 1997) show a smaller reaction to CCK agonists. Benzodiacepines such as lorazepam and alprazolam have also shown to attenuate the effects produced by CCK-4 administration in healthy volunteers (de Montigny 1989; Zwanzger, Eser et al. 2003). The GABA neurotransmission enhancing drugs tiagabine and vigabatrin given for 1 week also reduce the vulnerability of healthy volunteers to $50 \mu \mathrm{g}$ of CCK-4 (Zwanzger, Baghai et al. 2001; Zwanzger, Eser et al. 2003), although these drugs have yet to show therapeutic usefulness in PD patients (Mula, Pini et al. 2007). Interestingly, an acute exercise session of 30 minutes can protect healthy volunteers from CCK induced panic attacks when compared with 30 minutes of rest (Strohle, Feller et al. 2005). This is consistent with the therapeutic effects of an aerobic exercise program in PD patients (Broocks, Bandelow et al. 1998).

\section{Voluntary Hyperventilation}

\section{Background}

It was found that a hyperventilation-driven reduction in $\mathrm{PCO} 2$ is associated with vulnerability to sodium lactate induced panic attacks (Gorman, Goetz et al. 1988). Moreover, there's an overlap in panic symptoms and the symptoms produced by acute respiratory alkalosis. Based on these findings, a VH model for panic was developed (Lum 1987). Although hyperventilation is now largely disregarded as a cause of panic, the practical and non-invasive features of $\mathrm{VH}$ yielded a compelling and large amount of research (Maddock 2001).

\section{Features \& technique}

Many different methods of $\mathrm{VH}$ have been used across laboratories. However, most techniques of $\mathrm{VH}$ involve visual or auditory stimuli to indicate the frequency of 30 breathing cycles per minute for 4 to15 minutes (Gorman, Papp et al. 1994; Papp, Martinez et al. 1997; Nardi, Valenca et al. 2001) or visual feed-back for the subject to breathe as necessary to keep an end-tidal pCO2 lower than $20 \mathrm{mmHg}$ for 8 minutes (Maddock and Mateo-Bermudez 1990; Maddock and Carter 1991). Notably, one laboratory used a long tube attached to a mask to increase dead space and maintain baseline levels of $\mathrm{pCO} 2$ as control isocapnic hyperventilation (Zandbergen, Lousberg et al. 1990). 
Introduction: Experimental models of panic

Most techniques used in $\mathrm{VH}$ will usually produce drop in pCO2 below $20 \mathrm{mmHg}$ in less than 2 minutes (Wilhelm, Gerlach et al. 2001; Friedman, Mathis et al. 2006). After hyperventilation pCO2 levels will recover slowly probably due to the typical accumulation of systemic and brain lactate during VH (Posse, Dager et al. 1997) that is probably mediated by the effects of respiratory alkalosis on carbohydrate metabolism (Siesjo 1982; Maddock and Mateo-Bermudez 1990). In contrast to an inhalation with CO2, VH and the subsequent drop in pCO2 induces a reduction in cerebral blood velocity (van Rijen, Luyten et al. 1989). To our knowledge there is no systematic study on the relationship between the panicogenic effects of $\mathrm{VH}$ and HPA axis activation. Nevertheless, one report suggests that $\mathrm{VH}$ may lead to a mild increase in cortisol and plasma catecholamines after 10 minutes (Laderach and Straub 2001) probably related to respiratory work and skeletal muscle contraction.

\section{Underlying mechanisms}

Hyperventilation has once been regarded not only as an experimental maneuver to induce panic but also the cause of naturalistic panic attacks (Lum 1987; Ley 1992). This idea of a causative role emphasizes on the sequelae of hyperventilation in the production of mild physical symptoms that in a cognitive vicious circle will render the development of full-blown panic. However, against this theory lays the fact that $\mathrm{VH}$ is a relatively weak and inconsistent method to induce panic (Zandbergen, Lousberg et al. 1990; Gorman, Papp et al. 1994). Studies that monitor pCO2 levels of subjects during the recovery phase of $\mathrm{VH}$ show that panic-responsive PD patients have larger decreases and a longer recovery (Maddock and Carter 1991; Wilhelm, Gerlach et al. 2001). This has lead to the suggestion that VH may produce a more downward adjustment in the $\mathrm{CO} 2$ sensitivity of PD patients who then become more vulnerable to the rising than the absolute levels of CO2 (Wilhelm, Gerlach et al. 2001).

Another proposed mechanism links VH with panic provocation induced by lactate. Acute and disproportionate brain lactate elevations are seen in $\mathrm{PD}$ after $\mathrm{VH}$ as measured with 1H-NMR spectroscopy techniques (Dager, Strauss et al. 1995). The proposed mechanisms behind this exaggerated lactate response to alkalotic challenges (including sodium lactate infusions) involve: hypoxia due to vasoconstriction, a disturbance in intracellular $\mathrm{pH}$ regulation and enhanced glycolysis due to adrenergic activation (Maddock 2001). In this regard, PD patients have shown a more pronounced reduction basilar artery flow during $\mathrm{VH}$ than healthy controls (Gibbs 1992; Ball and Shekhar 1997).

Validity

The voluntary nature of hyperventilation techniques makes this maneuver very safe. The usual side effects such as dizziness and paresthesia are within the characteristic symptoms of a panic attack, but are usually present in all subjects that undergo $\mathrm{VH}$, regardless of the 
subject's condition or challenge outcome. According to studies using a large sample of $\mathrm{VH}$-sensitive PD patients, the frequencies of DSM-IV panic attack symptoms provoked by the maneuver were similar to those reported during the patients spontaneous panic attacks (Nardi, Lopes et al. 2004). In a Dutch sample of 48 patients with PD, only $21 \%$ of subjects panicked during $\mathrm{VH}$, but recognized the induced panic symptoms to be similar to their spontaneous panic attacks (Spinhoven, Onstein et al. 1992). In conflict with this, previous reports are consistent in that $\mathrm{VH}$ produces an increase in somatic symptoms but no change in subjective measures of panic (Griez, Zandbergen et al. 1988; Maddock and Carter 1991).

A Brazilian group lead by Nardi $(2001 ; 2001 ; 2002)$ published a series of studies that provide evidence towards a considerable discriminative power of $\mathrm{VH}$ when the panic response rates in PD patients are compared to those with major depression, OCD and SP. Combining a number of their studies, Nardi and coworkers. (2000; 2001a; 2001b; 2002) have shown that 44 up till $64 \%$ of the subjects respond with a panic attack using $\mathrm{VH}$ as a challenge technique. Although other laboratories (Maddock, Carter et al. 1991; Wilhelm, Gerlach et al. 2001) report similar rates with fewer patients, other groups have found $\mathrm{VH}$ to have a very low sensitivity when compared to the rates of $\mathrm{CO} 2$ induced panic in the same group of patients (Zandbergen, Lousberg et al. 1990; Papp, Martinez et al. 1997). Conclusions from these latter studies are that $\mathrm{VH}$ is, at best, a weak panicogen when compared to $\mathrm{CO} 2$. Overall, the symptom convergence and panic rates of $\mathrm{VH}$ in $\mathrm{PD}$ across laboratories are difficult to interpret because of differences in subject selection, procedures used for hyperventilation and operational criteria used to assess the occurrence of a panic attack. With regard to reliability, one study that compared the type and severity of $\mathrm{VH}$ elicited symptoms reported by $14 \mathrm{PD}$ patients and 14 healthy control subjects on two occasions, one week apart, revealed test-retest consistency (Lindsay, Saqi et al. 1991). To our knowledge, no data support a clinical validation for $\mathrm{VH}$ as an experimental model for panic attacks as there are no systematic studies that test the $\mathrm{VH}$ challenge after the use of an anti-panic maneuver or treatment.

\section{Doxapram}

\section{Background}

Doxapram is a CNS stimulant (analeptic) commonly used in the management of respiratory failure. The capability of intravenous doxapram to induce hyperventilation and a consecutive drop in pCO2 inspired its use as a model for panic (Lee, Curtis et al. 1993).

\section{Features \& technique}

In panic provocation protocols, doxapram hydrochloride is administered as a $0.5 \mathrm{mg} / \mathrm{kg}$ dose in $10 \mathrm{~mL}$ of saline over 15 seconds (Gutman, Coplan et al. 2005). As a respiratory stimulant, 
doxapram produces an increase in ventilation and a drop in pCO2 of about $5 \%$ in healthy subjects and up till $20 \%$ in PD patients (Lee, Curtis et al. 1993). Whether the HPA-axis is activated after doxapram administration is not clear (Gutman, Coplan et al. 2005; Abelson, Khan et al. 2006). In any case, the activation is unspecific and unrelated to the panic response (Gutman, Coplan et al. 2005; Abelson, Khan et al. 2006).

\section{Underlying mechanisms}

One proposed explanation states that doxapam (As well as most other panicogenic substances) produces physiological arousal, which in susceptible individuals leads to misinterpretation of bodily sensations, ultimately with stimulation of the amygdala and production of panic (Gutman, Coplan et al. 2005). Some research provides elements to link doxapram to the same mechanisms involved in $\mathrm{CO} 2$ induced panic. Doxapram could exert its effect by the inhibition of TASK-1 and TASK-3 (Cotten, Keshavaprasad et al. 2006), which are $\mathrm{pH}$ sensitive potassium channels expressed in brainstem serotonergic neurons Washburn, Sirois et al. 2002). By inhibition of these channels, doxapram may increase the excitability of brainstem $\mathrm{CO} 2$ sensitive neurons that potentially warn the organism of detrimental effects of asphyxia.

\section{Validity}

Doxapram can be regarded as safe when used at the conventional panicogenic dosages. However, its use should be restricted in subjects with risk of seizures or pharmacotherapy with MAOI's. The validity of doxapram as a model for panic is limited due to the lack of studies. In any case, doxapram seems to be a potent panicogen with panic rates ranging from 80 to 100 $\%$ in PD patients and 0 to $20 \%$ in healthy control subjects (Lee, Curtis et al. 1993; Gutman, Coplan et al. 2005; Kent, Coplan et al. 2005).

Other experimental models of panic

\section{Flumazenil}

Flumazenil is a benzodiazepine receptor antagonist used in research and in clinical practice for the management of benzodiazepine overdose. As an intravenous dose of $2 \mathrm{mg}$ flumazenil is generally devoid of any significant symptomatic effect but may produce panic attack in PD patients (Nutt, Glue et al. 1990). This finding put forward the interesting hypothesis that PD patients may have an abnormal benzodiazepine receptor set point. A study performed by the Bristol group suggests that flumazenil has a robust panicogenic effect that can be blocked with standard antipanic treatment with SSRI's (Bell, Forshall et al. 2002). Although two studies have not replicated the previously described findings in PD patients (Strohle, Kellner et al. 
1998; Strohle, Kellner et al. 1999), methodological differences may account for these results (Potokar, Lawson et al. 1999). The use of this model in panic has been very limited and further validation studies are timely.

\section{Caffeine}

Caffeine is an adenosine receptor antagonist with widespread use as a stimulant. Although caffeine has been extensively used as a model for panic (Bourin, Baker et al. 1998) its rather unspecific effects are probably better related to generalized anxiety than to panic (Bruce, Scott et al. 1992). Additionally, to our knowledge no clinical validation studies have been performed and in the last decade the amount of research using caffeine as a model of panic has been scarce. However, recent studies suggest that PD patients that panic after caffeine administration are associated with having respiratory subtype panic attacks and are more sensitive to CO2 (Masdrakis, Papakostas et al. 2007; Nardi, Valenca et al. 2007).

Adrenergic challenges: Yohimbine, Isoprotenerol and Epinephrine

In general, very little work has been done with noradrenergic challenges as models for panic in the last decade. Yohimbine is a $\alpha 2$-antagonist that is thought to produce panic symptoms by increasing activity in the locus ceruleus (Bourin, Baker et al. 1998). Yohimbine has shown to provoke panic attacks in PD patients at a higher rate compared with healthy subjects (Charney, Heninger et al. 1984). Additionally, patients with GAD or OCD are not panic sensitive to the $\alpha 2$-antagonist challenge (Rasmussen, Goodman et al. 1987; Charney, Woods et al. 1989). On the other hand, two factors make yohimbine a questionable model for panic: (i) yohimbine elicits flashbacks and panic attacks in patients with PTSD (Southwick, Krystal et al. 1997) and (ii) standard antipanic treatment with imipramine does not reduce the effects of the challenge in patients with PD (Charney and Heninger 1985).

isoprotenerol is a peripherally active $\beta$-agonist that potentially induces panic attacks that are phenomenologically similar to those produced by a sodium lactate infusion and placebo dextrose infusions in PD patients (Balon, Yeragani et al. 1988). A challenge with isoprotenerol produces panic attacks in a greater proportion of PD patients as opposed to healthy volunteers (Pohl, Yeragani et al. 1988), which can be reduced by standard antipanic treatment with imipramine (Pohl, Yeragani et al. 1990). Other studies that validate isoprotenerol as a model of panic are scant. In the case of epinephrine, very few studies address the validity of this model (Veltman, van Zijderveld et al. 1996).

\section{Serotonergic panicogens: $d$-Fenfluramine and $m C P P$}

Fenfluramine, an acute 5-HT enhancer, has been describe to provoke more intense panic and anxiety in patients with PD than patients with major depressive disorder and healthy volunteers 
Introduction: Experimental models of panic

(Targum and Marshall 1989). Nevertheless, these findings have been questioned as to whether they better account for anticipatory anxiety or generalized anxiety (Hollander, Liebowitz et al. 1990). In fact, fenfluramine premedication has shown to increase anxiety and reduce the panicogenic effects of $\mathrm{CO} 2$ in patients with PD (Mortimore and Anderson 2000). These latter findings are explained in the context of a theory that attributes a dual role for $5-\mathrm{HT}$ in anxiety (Deakin and Graeff 1991).

Metachlorophenylpiperazine (m-CPP) is mainly a $5-\mathrm{HT}$ receptor agonist, however it also antagonizes 5-HT3 and $\alpha 1$-adrenergic receptors (Kahn and Wetzler 1991). As a model for panic results are not clear: (i) its panic effect depends on the route of administration, (ii) the challenge can exacerbate OCD symptoms in patients with this disorder (Erzegovesi, Martucci et al. 2001) and anger and anxiety in GAD patients (Germine, Goddard et al. 1992), (iii) findings are difficult to interpret as other 5-HT enhancers such as L-5 hydroxytyptophan not only does not provoke panic but reduces the effects of a $35 \% \mathrm{CO} 2$ challenge in patients with PD and healthy volunteers (Schruers, van Diest et al. 2002).

\section{Conclusions}

The use of experimental models has aided in our understanding of panic and fear. Together with animal studies, these models can help delineate the neurochemical and neuroanatomical underpinnings of defensive behavior. In any case, even a cautious interpretation of the modest evidence supports the assumption that pathological anxiety does not rely on one single mechanism.

This complexity is manifest when comparing the most well studied panicogens (i.e. lactate, $\mathrm{CO} 2$ and $\mathrm{CCK}$ ) in the degree to which they meet the criteria for an ideal experimental model. Apparently, the vulnerability triggered by the different models of panic does not completely overlap, even though they may produce similar symptoms. For instance subjects with GAD or OCD are not affected by a CO2 challenge or a lactate infusion in the same way as PD patients. In contrast, the studies published so far strongly suggest that CCK is as panicogenic in GAD and in OCD as it is in PD. This differential sensitivity, if confirmed, may pave the way for further pharmacologic dissection of defensive behavior.

Other experimental models are still in need of further validation. Voluntary hyperventilation has been recently fairly validated, however the blockablity of the maneuver has not been systematically tested with standard anti-panic interventions. Moreover, the evidence so far suggests it is a weak panicogen when compared to CO2. Doxapram is a promising agent that may meet criteria for an ideal experimental model provided additional validation.

Much work is still to be done. Experimental models of panic and fear can eventually be useful paradigms for proof-of-concept studies. Since the traditional ways of measuring the 
Chapter 1

panic response to the different models depend on the conceptual cortico-linguistic abilities of the subjects being tested, there is a need to search for objective physiological measurements (e.g. cardiovascular, neuroendocrine, etc) that can predict a challenge response. This may be relevant in the search for biomarkers that are more objective and scientifically useful for studies into the genetic and neuroanatomical determinants of panic and fear susceptibility. This altogether may be relevant for better understanding of etiological mechanisms and accordingly, call for a revised nosology. Experimental challenges may finally prove useful in suggesting effective treatments for discrete anxiety disorders. 
Introduction: Experimental models of panic

\section{References}

Abelson, J. L., S. Khan, et al. (2006). "HPA axis activity in patients with panic disorder: review and synthesis of four studies." Depress Anxiety 24(1): 66-76.

Abelson, J. L. and R. M. Nesse (1994). "Pentagastrin infusions in patients with panic disorder. I. Symptoms and cardiovascular responses." Biol Psychiatry 36(2): 73-83.

Abelson, J. L., R. M. Nesse, et al. (1994). "Pentagastrin infusions in patients with panic disorder. II. Neuroendocrinology." Biol Psychiatry 36(2): 84-96.

Andreatini, R., C. Blanchard, et al. (2001). "The brain decade in debate: II. Panic or anxiety? From animal models to a neurobiological basis." Braz J Med Biol Res 34(2): 145-54.

Argyropoulos, S. V., J. E. Bailey, et al. (2002). "Inhalation of $35 \%$ CO(2) results in activation of the HPA axis in healthy volunteers." Psychoneuroendocrinology 27(6): 715-29.

Bailey, J. E., S. V. Argyropoulos, et al. (2005). "Behavioral and cardiovascular effects of $7.5 \% \mathrm{CO} 2$ in human volunteers." Depress Anxiety 21(1): 18-25.

Bailey, J. E., S. V. Argyropoulos, et al. (2003). "Does the brain noradrenaline network medlate the effects of the $\mathrm{CO} 2$ challenge?" J Psychopharmacol 17(3): 252-9.

Bailey, J. E., A. Kendrick, et al. (2007). "A validation of the 7.5\% CO2 model of GAD using paroxetine and lorazepam in healthy volunteers." J Psychopharmacol 21(1): 42-9.

Ball, S. and A. Shekhar (1997). "Basilar artery response to hyperventilation in panic disorder." Am J Psychiatry 154(11): 1603-4.

Balon, R., V. K. Yeragani, et al. (1988). "Phenomenological comparison of dextrose, lactate, and isoproterenol associated panic attacks." Psychiatry Res 26(1): 43-50.

Battaglia, M. and G. Perna (1995). "The 35\% CO2 challenge in panic disorder: optimization by receiver operating characteristic (ROC) analysis." J Psychiatr Res 29(2): 111-9.

Bell, C., S. Forshall, et al. (2002). "Does 5-HT restrain panic? A tryptophan depletion study in panic disorder patients recovered on paroxetine." J Psychopharmacol 16(1): 5-14.

Bellodi, L., G. Perna, et al. (1998). "CO2-induced panic attacks: a twin study." Am J Psychiatry 155(9): 1184-8.

Bertoglio, L. J., V. C. de Bortoli, et al. (2007). "Cholecystokinin-2 receptors modulate freezing and escape behaviors evoked by the electrical stimulation of the rat dorsolateral perlaqueductal gray." Brain Res.

Bickler, P. E., L. Litt, et al. (1988). "Effects of acetazolamide on cerebral acid-base balance." J App| Physiol 65(1): 422-7.

Blanchard, D. C., G. Griebel, et al. (2003). "The Mouse Defense Test Battery: pharmacological and behavioral assays for anxiety and panic." Eur J Pharmacol 463(1-3): 97-116.

Bourin, M., G. B. Baker, et al. (1998). "Neurobiology of panic disorder." J Psychosom Res 44(1): 163-80.

Bouton, M. E., S. Mineka, et al. (2001). "A modern learning theory perspective on the etiology of panic disorder." Psychol Rev 108(1): 4-32.

Bradwejn, J. and D. Koszycki (1994). "Imipramine antagonism of the panicogenic effects of cholecystokinin tetrapeptide in panic disorder patients." Am J Psychiatry 151(2): 261-3.

Bradwejn, J., D. Koszycki, et al. (1992). "A dose-ranging study of the behavioral and cardiovascular effects of CCK-tetrapeptide in panic disorder." Bjol Psychiatry 32(10): 903-12.

Bradwejn, J., D. Koszycki, et al. (1991). "Dose ranging study of the effects of cholecystokinin in healthy volunteers." J Psychiatry Neurosci 16(2): 91-5.

Bradwejn, J., D. Koszycki, et al. (1994). "Effects of flumazenil on cholecystokinin-tetrapeptide-induced panic symptoms in healthy volunteers." Psychopharmacology (Berl) 114(2): 257-61.

Bradwejn, J., D. Koszycki, et al. (1994). "The panicogenic effects of cholecystokinin-tetrapeptide are antagonized by $L-365,260$, a central cholecystokinin receptor antagonist, in patients with panic disorder." Arch Gen Psychiatry 51(6): 486-93.

Bradwejn, J., D. Koszycki, et al. (1990). "Cholecystokinin-tetrapeptide induces panic attacks in patients with panic disorder." Can J Psychiatry 35(1): 83-5.

Bradwejn, J., D. Koszycki, et al. (1995). "Effect of Cl-988 on cholecystokinin tetrapeptide-induced panic symptoms in healthy volunteers." Biol Psychiatry 38(11): 742-6. 
Bradwejn, J., D. Koszycki, et al. (1992). "Replication of action of cholecystokinin tetrapeptide in panic disorder: clinical and behavioral findings." Am J Psychiatry 149(7): 962-4.

Bradwejn, J., J. M. LeGrand, et al. (1998). "Effects of cholecystokinin tetrapeptide on respiratory function in healthy volunteers." Am J Psychiatry 155(2): 280-2.

Brawman-Mintzer, O., R. B. Lydiard, et al. (1997). "Effects of the cholecystokinin agonist pentagastrin in patients with generalized anxiety disorder." Am J Psychiatry 154(5): 700-2.

Briggs, A. C., D. D. Stretch, et al. (1993). "Subtyping of panic disorder by symptom profile." $\mathrm{Br} J$ Psychiatry 163: 201-9.

Broocks, A., B. Bandelow, et al. (1998). "Comparison of aerobic exercise, clomipramine, and placebo in the treatment of panic disorder." Am J Psychiatry 155(5): 603-9.

Bruce, M., N. Scott, et al. (1992). "Anxiogenic effects of caffeine in patients with anxiety disorders." Arch Gen Psychiatry 49(11): 867-9.

Buller, R., U. von Bardeleben, et al. (1989). "Specificity of lactate response in panic disorder, panic with concurrent depression and major depression." J Affect Disord 16(2-3): 109-13.

Canteras, N. S. (2002). "The medial hypothalamic defensive system: hodological organization and functional implications." Pharmacol Biochem Behav 71(3): 481-91.

Carr, D. B. and D. V. Sheehan (1984). "Panic anxiety: a new biological model." J Clin Psychiatry 45(8): 323-30.

Charney, D. S. (2003). "Neuroanatomical circuits modulating fear and anxiety behaviors." Acta Psychiatr Scand Suppl(417): 38-50.

Charney, D. S. and G. R. Heninger (1985), "Noradrenergic function and the mechanism of action of antianxiety treatment. II. The effect of long-term imipramine treatment." Arch Gen Psychiatry 42(5): 473-81.

Charney, D. S. and G. R. Heninger (1986). "Abnormal regulation of noradrenergic function in panic disorders. Effects of clonidine in healthy subjects and patients with agoraphobia and panic disorder." Arch Gen Psychiatry 43(11): 1042-54.

Charney, D. S., G. R. Heninger, et al. (1984). "Noradrenergic function in panic anxiety. Effects of yohimbine in healthy subjects and patients with agoraphobia and panic disorder." Arch Gen Psychiatry 41 (8): $751-63$.

Charney, D. S., S. W. Woods, et al. (1989). "Noradrenergic function in generalized anxiety disorder: effects of yohimbine in healthy subjects and patients with generalized anxiety disorder." Psychiatry Res 27(2): 173-82.

Cohen, M. E. and P. D. White (1951). "Life situations, emotions, and neurocirculatory asthenia (anxiety neurosis, neurasthenia, effort syndrome)." Psychosom Med 13(6): 335-57.

Coplan, J. D., R. Goetz, et al. (1998). "Plasma cortisol concentrations preceding lactate-induced panic. Psychological, biochemical, and physiological correlates." Arch Gen Psychiatry 55(2): 130-6.

Coplan, J. D., M. R. Liebowitz, et al. (1992). "Noradrenergic function in panic disorder. Effects of intravenous clonidine pretreatment on lactate induced panic." Biol Psychiatry 31(2): 135-46.

Corfield, D. R., G. R. Fink, et al. (1995). "Evidence for limbic system activation during CO2-stimulated breathing in man." J Physiol 488 ( Pt 1): 77-84.

Cotten, J. F., B. Keshavaprasad, et al. (2006). "The ventilatory stimulant doxapram inhibits TASK tandem pore (K2P) potassium channel function but does not affect minimum alveolar anesthetic concentration." Anesth Analg 102(3): 779-85.

Cowley, D. S., S. R. Dager, et al. (1986). "Lactate-induced panic in primary affective disorder." Am J Psychiatry 143(5): 646-8.

Cowley, D. S., S. R. Dager, et al. (1988). "Response to lactate infusion in generalized anxiety disorder." Biol Psychiatry 24(4): 409-14.

Cowley, D. S., S. R. Dager, et al. (1991). "Lactate vulnerability after alprazolam versus placebo treatment of panic disorder." Biol Psychiatry 30(1): 49-56.

Dager, S. R., S. D. Friedman, et al. (1999). "Two-dimensional proton echo-planar spectroscopic imaging of brain metabolic changes during lactate-induced panic." Arch Gen Psychiatry 56(1): 70-7.

Dager, S. R., T. Richards, et al. (1997). "Single-voxel 1H-MRS investigation of brain metabolic changes during lactate-induced panic." Psychiatry Res 76(2-3): 89-99. 
Dager, S. R., W. L. Strauss, et al. (1995). "Proton magnetic resonance spectroscopy investigation of hyperventilation in subjects with panic disorder and comparison subjects." Am J Psychiatry 152(5): 666-72.

de Leeuw, A. S., J. A. Den Boer, et al. (1996). "Pentagastrin has panic-inducing properties in obsessive compulsive disorder." Psychopharmacologv (Berl) 126(4): 339-44.

de Montigny, C. (1989). "Cholecystokinin tetrapeptide induces panic-like attacks in healthy volunteers. Preliminary findings." Arch Gen Psychiatry 46(6): 511-7.

Deakin, J. F. W. and F. G. Graeff (1991). "5-HT and mechanisms of defence." JPsychopharmacol 5(4): 305-315.

Dillon, D. J., J. M. Gorman, et al. (1987). "Measurement of lactate-induced panic and anxiety." Psychiatry Res 20(2): 97-105.

Ehlers, A., J. Margraf, et al. (1986). "Lactate infusions and panic attacks: do patients and controls respond differently?" Psychiatry Res 17(4): 295-308.

Erzegovesi, S., L. Martucci, et al. (2001). "Low versus standard dose mCPP challenge in obsessivecompulsive patients." Neuropsychopharmacology 24(1): 31-6.

Eser, D., C. Schule, et al. (2007). "Evaluation of the CCK-4 model as a challenge paradigm in a population of healthy volunteers within a proof-of-concept study." Psychopharmacology (Berl).

Facchinetti, F., G. Romano, et al. (1992). "Lactate infusion induces panic attacks in patients with premenstrual syndrome." Psychosom Med 54(3): 288-96.

Friedman, S. D., C. M. Mathis, et al. (2006). "Brain pH response to hyperventilation in panic disorder: preliminary evidence for altered acid-base regulation." Am J Psychiatry 163(4): 710-5.

Fyer, M. R., J. Uy, et al. (1987). "CO2 challenge of patients with panic disorder." Am J Psychiatry 144(8): 1080-2.

Ganong, W. (1997). Review of Medical Physiology. Stamford, Connecticut, Appleton and Lange.

George, D. T., P. Glue, et al. (1990). "Lactate-induced electrolyte changes in the cerebrospinal fluid of rabbits." Biol Psychiatry 27(1): 104-8.

George, D. T., T. Lindquist, et al. (1995). "Effect of chloride or glucose on the incidence of lactate-induced panic attacks." Am J Psychiatry 152(5): 692-7.

George, D. T., D. J. Nutt, et al. (1989). "Lactate and hyperventilation substantially attenuate vagal tone in normal volunteers. A possible mechanism of panic provocation?" Arch Gen Psychiatry 46(2): $153-6$.

Germine, M., A. W. Goddard, et al. (1992). "Anger and anxiety responses to m-chlorophenylpiperazine in generalized anxiety disorder." Biol Psychiatry 32(5): 457-61.

Gibbs, D. M. (1992). "Hyperventilation-induced cerebral ischemia in panic disorder and effect of nimodipine." Am J Psychiatry 149(11): 1589-91.

Goetz, R. R., D. F. Klein, et al. (1994). "Consistencies between recalled panic and lactate-induced panic." Anxiety 1(1): 31-6.

Gorman, J. M. (2003). "Does the brain noradrenaline network mediate the effects of the CO2 challenge?" JPsychopharmacol 17(3): 265-6; discussion 267-8.

Gorman, J. M., J. Askanazi, et al. (1984). "Response to hyperventilation in a group of patients with panic disorder." Am J Psychiatry 141(7): 857-61.

Gorman, J. M., D. Battista, et al. (1989). "A comparison of sodium bicarbonate and sodium lactate infusion in the induction of panic attacks." Arch Gen Psychiatry 46(2): 145-50.

Gorman, J. M., R. R. Goetz, et al. (1990). "Sodium D-lactate infusion of panlc disorder patients." Neuropsychopharmacology 3(3): 181-9.

Gorman, J. M., R. R. Goetz, et al. (1988). "Hyperventilation occurs during lactate-induced panic." Journal of Anxiety Disorders 2(3): 193-202.

Gorman, J. M., J. M. Kent, et al. (2000). "Neuroanatomical hypothesis of panic disorder, revised." Am J Psychiatry 157(4): 493-505.

Gorman, J. M., G. F. Levy, et al. (1983). "Effect of acute beta-adrenergic blockade on lactate-induced panic." Arch Gen Psychiatry 40(10): 1079-82.

Gorman, J. M., L. Papp, et al. (1990). Biological models of panic disorder. Handbook of anxiety. R. Noyes, M. Roth and G. D. Burrows. Amsterdam; New York, Elsevier ; New York, NY III: The neurobiology of anxlety. 
Gorman, J. M., L. A. Papp, et al. (1993). "The effect of acetazolamide on ventilation in panic disorder patients." Am J Psychiatry 150(10): 1480-4.

Gorman, J. M., L. A. Papp, et al. (1994). "Anxiogenic effects of CO2 and hyperventilation in patients with panic disorder." Am J Psychiatry 151 (4): 547-53.

Graeff, F. G. (2004). "Serotonin, the periaqueductal gray and panic." Neurosci Biobehav Rev 28(3): 23959.

Graeff, F. G., C. Garcia-Leal, et al. (2005). "Does the panic attack activate the hypothalamic-pituitaryadrenal axis?" An Acad Bras Cienc 77(3): 477-91.

Griez, E., C. de Loof, et al. (1990). "Specific sensitivity of patients with panic attacks to carbon dioxide inhalation." Psychiatry Res 31(2): 193-9.

Griez, E., M. A. van den Hout, et al. (1987). "Body fluids after CO2 inhalation: insight into panic mechanisms?" Eur Arch Psychiatry Neurol Sci 236(6): 369-71.

Griez, E., J. Zandbergen, et al. (1988). "Effects of low pulmonary CO2 on panic anxiety." Compr Psychiatry 29(5): 490-7.

Griez, E. J., A. Colasanti, et al. (2007). "Carbon dioxide inhalation induces dose-dependent and agerelated negative affectivity." PLOS ONE 2(10): e987.

Griez, E. J., H. Lousberg, et al. (1987). "CO2 vulnerability in panic disorder." Psychiatry Res 20(2): 87-95.

Griez, E. J. L. (2001). Anxiety disorders: an introduction to clinical management and research. Chichester; New York, Wiley.

Gutman, D. A., J. Coplan, et al. (2005). "Doxapram-induced panic attacks and cortisol elevation." Psychiatry Res 133(2-3): 253-61.

Guttmacher, L. B., D. L. Murphy, et al. (1983). "Pharmacologic models of anxiety." Compr Psychiatry 24(4): 312-26.

Hollander, E., M. R. Liebowitz, et al. (1990). "Fenfluramine, cortisol, and anxiety." Psychiatry Res 31 (2): $211-3$.

Hollander, E., M. R. Liebowitz, et al. (1989). "Cortisol and sodium lactate-induced panic." Arch Gen Psychiatry 46(2): 135-40.

Holt, P. E. and G. Andrews (1989). "Provocation of panic: three elements of the panic reaction in four anxiety disorders." Behav Res Ther 27(3): 253-61.

Javanmard, M., J. Shlik, et al. (1999). "Neuroanatomic correlates of CCK-4-induced panic attacks in healthy humans: a comparison of two time points." Biol Psychiatry 45(7): 872-82.

Jensen, C. F., T. W. Keller, et al. (1997). "Behavioral and neuroendocrine responses to sodlum lactate infusion in subjects with posttraumatic stress disorder." Am J Psychiatry 154(2); 266-8.

Jensen, K. E., C. Thomsen, et al. (1988). "In vivo measurement of intracellular $\mathrm{pH}$ in human brain during different tensions of carbon dioxide in arterial blood. A 31P-NMR study." Acta Physiol Scand 134(2): 295-8.

Kahn, R. S. and S. Wetzler (1991). "m-Chlorophenylpiperazine as a probe of serotonin function." Biol Psychiatry 30(11): 1139-66.

Katzman, M. A., D. Koszycki, et al. (2004). "Effects of CCK-tetrapeptide in patients with social phobia and obsessive-compulsive disorder." Depress Anxiety 20(2): 51-8.

Kaye, J., F. Buchanan, et al. (2004). "Acute carbon dioxide exposure in healthy adults: evaluation of a novel means of investigating the stress response." J Neuroendocrinol 16(3): 256-64.

Kelliner, M., L. Herzog, et al. (1995). "Possible role of atrial natriuretic hormone in pituitary-adrenocortical unresponsiveness in lactate-induced panic." Am J Psychiatry 152(9): 1365-7.

Kent, J. M., J. D. Coplan, et al. (2005). "Prediction of panic response to a respiratory stimulant by reduced orbitofrontal cerebral blood flow in panic disorder." Am J Psychiatry 162(7): 1379-81.

Khan, S., I. Liberzon, et al. (2004). "Effects of propranolol on symptom and endocrine responses to pentagastrin." Psychoneuroendocrinology 29(9): 1163-71.

King, M. S. and A. J. Baertschi (1992). "Ventral pontine catecholaminergic pathway mediates the vasopressin response to splanchnic osmostimulation in conscious rats." Brain Res 580(1-2): 81-91.

Klein, D. F. (1993). "False suffocation alarms, spontaneous panics, and related conditions. An integrative hypothesis." Arch Gen Psychiatry 50(4): 306-17. 
Introduction: Experimental models of panic

Klein, D. F. and D. C. Ross (1986). "Response of panic patients and normal controls to lactate infuslons." Psychiatry Res 19(2): 163-7.

Kondoh, Y., M. Kawase, et al. (1992). "D-lactate concentrations in blood, urine and sweat before and after exercise." Eur J Appl Physiol Occup Physiol 65(1): 88-93.

Koszycki, D., R. M. Zacharko, et al. (1998). "Behavioral, cardiovascular, and neuroendocrine profiles following CCK-4 challenge in healthy volunteers: a comparison of panickers and nonpanickers." Depress Anxiety 8(1): $1-7$.

Kramer, M. S., N. R. Cutler, et al. (1995). "A placebo-controlled trial of $L-365,260$, a CCKB antagonist, in panic disorder." Biol Psychiatry 37(7): 462-6.

Laderach, H. and W. Straub (2001). "Effects of voluntary hyperventilation on glucose, free fatty acids and several glucostatic hormones." Swiss Med Wkly 131(1-2): 19-22.

LeDoux, J. E. (1996). The emotional brain: the mysterious underpinnings of emotional life. New York, Simon \& Schuster.

Lee, Y. J., G. C. Curtis, et al. (1993). "Panic attacks induced by doxapram." Biol Psychiatry 33(4): 295-7.

Leverve, X. M. and I. Mustafa (2002). "Lactate: A key metabolite in the intercellular metabolic interplay." Crit Care 6(4): 284-5

Ley, R. (1992). "The many faces of Pan: psychological and physiological differences among three types of panic attacks." Behav Res Ther 30(4): 347-57.

Liebowitz, M. R., J. D. Coplan, et al. (1995). "Effects of intravenous diazepam pretreatment on lactateinduced panic," Psychiatry Res 58(2): 127-38.

Liebowitz, M. R., A. J. Fyer, et al. (1986). "Alprazolam in the treatment of panic disorders." $\underline{\mathrm{J} \text { Clin }}$ Psychopharmacol 6(1): 13-20.

Liebowitz, M. R., A. J. Fyer, et al. (1984). "Lactate provocation of panic attacks. I. Clinical and behavioral findings." Arch Gen Psychiatry 41(8): 764-70.

Liebowitz, M. R., J. M. Gorman, et al. (1986). "Possible mechanisms for lactate's induction of panic." Am J Psychiatry 143(4): 495-502.

Liebowitz, M. R., J. M. Gorman, et al. (1985). "Lactate provocation of panic attacks. II. Biochemical and physiological findings." Arch Gen Psychiatry 42(7): 709-19.

Lindsay, S., S. Saqi, et al. (1991). "The test-retest reliability of the hyperventilation provocation test." $\underline{J}$ Psychosom Res 35(2-3): 155-62.

Lines, C., J. Challenor, et al. (1995). "Cholecystokinin and anxiety in normal volunteers: an investigation of the anxiogenic properties of pentagastrin and reversal by the cholecystokinin receptor subtype B antagonist L-365,260." Br J Clin Pharmacol 39(3): 235-42.

Liotti, M., S. Brannan, et al. (2001). "Brain responses associated with consciousness of breathlessness (air hunger)." Proc Natl Acad Sci U S A 98(4): 2035-40.

Lum, L. C. (1987). "Hyperventilation syndromes in medicine and psychiatry: a review." JR Soc Med 80(4): 229-31.

Maddock, R. J. (2001). "The lactic acid response to alkalosis in panic disorder: an integrative review." $\underline{\mathrm{J}}$ Neuropsychiatry Clin Neurosci 13(1): 22-34.

Maddock, R. J, and C. S. Carter (1991). "Hyperventilation-induced panic attacks in panic disorder with agoraphobia." Biol Psychiatry 29(9): 843-54.

Maddock, R. J., C. S. Carter, et al. (1991). "Elevated serum lactate associated with panic attacks induced by hyperventilation." Psychiatry Res 38(3): 301-11.

Maddock, R. J. and J. Mateo-Bermudez (1990). "Elevated serum lactate following hyperventilation during glucose infusion in panic disorder." Biol Psychiatry 27(4): 411-8.

Maron, E., I. Toru, et al. (2004). "The effect of 5-hydroxytryptophan on cholecystokinin-4-induced panic attacks in healthy volunteers." J Psychopharmacol 18(2): 194-9.

Martel, F. L., C. Hayward, et al. (1999). "Salivary cortisol levels in socially phobic adolescent girls." Depress Anxiety $10(1): 25-7$.

Masdrakis, V. G., Y. G. Papakostas, et al. (2007). "Caffeine challenge in patients with panic disorder: baseline differences between those who panic and those who do not." Depress Anxiety.

Mathew, R. J. and W. H. Wilson (1988). "Cerebral blood flow changes induced by $\mathrm{CO} 2$ in anxlety." Psychiatry Res 23(3): 285-94. 
Chapter 1

Mathew, R. J., W. H. Wilson, et al. (1997). "Cerebral vasodilation and vasoconstriction associated with acute anxiety." Biol Psychiatry 41(7): 782-95.

Mathew, R. J., W. H. Wilson, et al. (1989). "Responses to hypercarbia induced by acetazolamide in panic disorder patients." Am J Psychiatry 146(8): 996-1000.

McCann, U. D., C. M. Morgan, et al. (1997). "Effects of the 5-HT3 antagonist, ondansetron, on the behavioral and physiological effects of pentagastrin in patients with panic disorder and social phobia." Neuropsychopharmacology 17(6): 360-9.

McCann, U. D., S. O. Slate, et al. (1997). "A comparison of the effects of intravenous pentagastrin on patients with social phobia, panic disorder and healthy controls." Neuropsychopharmacology 16(3): 229-37.

McNaughton, N. and P. J. Corr (2004). "A two-dimensional neuropsychology of defense: fear/anxiety and defensive distance." Neurosci Biobehav Rev 28(3): 285-305.

Mortimore, C. and I. M. Anderson (2000). "d-Fenfluramine in panic disorder; a dual role for 5hydroxytryptamine." Psychopharmacology (Berl) 149(3): 251-8.

Mukaddam-Daher, S., C. Lambert, et al. (1997). "Clonidine and ST-91 may activate imidazoline binding sites in the heart to release atrial natriuretic peptide." Hypertension 30(1 Pt 1): 83-7.

Mula, M., S. Pini, et al. (2007). "The role of anticonvulsant drugs in anxiety disorders: a critical review of the evidence." J Clin Psychopharmacol 27(3): 263-72.

Nakashima, K., T. Yamashita, et al. (1996). "The effect of sodium bicarbonate on CBF and intracellular pH in man: stable Xe-CT and 31P-MRS." Acta Neurol Scand Suppl 166: 96-8.

Nardi, A. E., F. L. Lopes, et al. (2004). "Psychopathological description of hyperventilation-induced panic attacks: a comparison with spontaneous panic attacks." Psychopathology 37(1): 29-35.

Nardi, A. E., I. Nascimento, et al. (2003). "Panic disorder in a breath-holding challenge test: a simple tool for a better diagnosis." Arg Neuropsiquiatr 61(3B): 718-22.

Nardi, A. E., A. M. Valenca, et al. (2007). "Caffeine and 35\% carbon dioxide challenge tests in panic disorder." Hum Psychopharmacol.

Nardi, A. E., A. M. Valenca, et al. (2006). "Psychopathological profile of 35\% CO2 challenge test-induced panic attacks: a comparison with spontaneous panic attacks." Compr Psychiatry 47(3): 20914.

Nardi, A. E., A. M. Valenca, et al. (2001). "Hyperventilation in panic disorder and social phobla." Psychopathology 34(3): 123-7.

Nardi, A. E., A. M. Valenca, et al. (2001). "Hyperventilation challenge test in panic disorder and depression with panic attacks." Psychiatry Res 105(1-2): 57-65.

Nardi, A. E., A. M. Valenca, et al. (2002). "Panic disorder and obsessive compulsive disorder in a hyperventilation challenge test." $\mathrm{J}$ Affect Disord 68(2-3): 335-40.

Netto, C. F. and F. S. Guimaraes (2004). "Anxiogenic effect of cholecystokinin in the dorsal periaqueductal gray." Neuropsychopharmacology 29(1): 101-7.

Nutt, D. J., P. Glue, et al. (1990). "Flumazenil provocation of panic attacks. Evidence for altered benzodiazepine receptor sensitivity in panic disorder." Arch Gen Psychiatry 47(10): 917-25.

Olson, B. R., M. Freillno, et al. (1993). "c-Fos Expression in Rat Brain and Brainstem Nuclei in Response to Treatments That Alter Food Intake and Gastric Motility." Molecular and Cellular Neuroscience 4(1): 93-106

Otte, C., S. Moritz, et al. (2007). "Blockade of the mineralocorticoid receptor in healthy men: effects on experimentally induced panic symptoms, stress hormones, and cognition." Neuropsychopharmacology 32(1): 232-8.

Pande, A. C., M. Greiner, et al. (1999). "Placebo-controlled trial of the CCK-B antagonist, Cl-988, in panic disorder." Biol Psychiatry 46(6): 860-2.

Pandit, J. J., R. M. Mohan, et al. (2003). "Cerebral blood flow sensitivity to $\mathrm{CO} 2$ measured with steadystate and Read's rebreathing methods." Respir Physiol Neurobiol 137(1): 1-10.

Papp, L. A., D. F. Klein, et al. (1993). "Diagnostic and substance specificity of carbon-dioxide-induced panic." Am J Psychiatry 150(2): 250-7.

Papp, L. A., J. M. Martinez, et al. (1997). "Respiratory psychophysiology of panic disorder: three respiratory challenges in 98 subjects." Am J Psychiatry 154(11): 1557-65. 
Parsons, L. M., G. Egan, et al. (2001). "Neuroimaging evidence implicating cerebellum in the experience of hypercapnia and hunger for air." Proc Natl Acad Sci U S A 98(4): 2041-6.

Perna, G., M. Battaglia, et al. (1994). "Carbon dioxide/oxygen challenge test in panic disorder." Psychiatry Res 52(2): 159-71.

Perna, G., A. Bertani, et al. (1997). "Modification of 35\% carbon dioxide hypersensitivity across one week of treatment with clomipramine and fluvoxamine: a double blind, randomized, placebocontrolled study." J Clin Psychopharmacol 17(3): 173-8.

Perna, G., R. Bussi, et al. (1999). "Sensitivity to $35 \%$ carbon dioxide in patients with generalized anxiety disorder." J Clin Psychiatry 60(6): 379-84.

Perna, G., S. Cocchi, et al. (1994). "Pharmacologic effect of toloxatone on reactivity to the $35 \%$ carbon dioxide challenge: a single-blind, random, placebo-controlled study." J Clin Psychopharmacol 14(6): 414-8.

Peskind, E. R., C. F. Jensen, et al. (1998). "Sodium lactate and hypertonic sodium chloride induce equivalent panic incidence, panic symptoms, and hypernatremia in panic disorder." Biol Psychiatry 44(10): 1007-16.

Pitts, F. N., Jr. and J. N. McClure, Jr. (1967). "Lactate metabolism in anxiety neurosis." $\underline{\text { N Engl J Med }}$ $277(25): 1329-36$.

Pohl, R., V. K. Yeragani, et al. (1990). "Effects of isoproterenol in panic disorder patients after antidepressant treatment." Biol Psychiatry 28(3): 203-14.

Pohl, R., V. K. Yeragani, et al. (1988). "Isoproterenol-induced panic attacks." Blol Psychiatry 24(8): $891-$ 902.

Pols, H. K. Verburg, et al. (1996). "Alprazolam premedication and 35\% carbon dioxide vulnerability in panic patients." Biol Psychiatry 40(9): 913-7.

Pols, H. J., R. C. Hauzer, et al. (1996). "Fluvoxamine attenuates panic induced by $35 \%$ CO2 challenge." $\underline{J}$ Clin Psychiatry 57(11): 539-42.

Posse, S., S. R. Dager, et al. (1997). "In vivo measurement of regional brain metabolic response to hyperventilation using magnetic resonance: proton echo planar spectroscopic imaging (PEPSI)." Magn Reson Med 37(6): 858-65.

Potokar, J., C. Lawson, et al. (1999). "Behavioral, neuroendocrine, and cardiovascular response to flumazenil: no evidence for an altered benzodiazepine receptor sensitivity in panic disorder." Biol Psychiatry 46(12): 1709-11.

Potts, N. L., J. R. Davidson, et al. (1991). "Levels of urinary free cortisol in social phobia." J Clin Psychiatry 52 Suppl: 41-2.

Prenoveau, J. M., J. P. Forsyth, et al. (2006). "Repeated exposure to $20 \% \mathrm{CO} 2$ challenge and risk for developing panic attacks: a controlled 6- and 12-month follow-up in a nonclinical sample." $\underline{\mathrm{J}}$ Anxiety Disord 20(8): 1158-67.

Raedler, T. J., H. Jahn, et al. (2006). "Megestrol attenuates the hormonal response to CCK-4-induced panic attacks." Depress Anxiety 23(3): 139-44.

Rasmussen, S. A., W. K. Goodman, et al. (1987). "Effects of yohimbine in obsessive compulsive disorder." Psychopharmacology (Berl) 93(3): 308-13.

Rassovsky, Y. and M. G. Kushner (2003). "Carbon dioxide in the study of panic disorder: issues of definition, methodology, and outcome." $\mathrm{J}$ Anxiety Disord 17(1): 1-32.

Richerson, G. B. (2004). "Serotonergic neurons as carbon dioxide sensors that maintain pH homeostasis." Nat Rev Neurosci 5(6): 449-61.

Sanderson, W. C., R. M. Rapee, et al. (1989). "The influence of an Illusion of control on panic attacks induced via inhalation of 5.5\% carbon dioxide-enriched air." Arch Gen Psychiatry 46(2): 157-62.

Schenberg, L. C., A. S. Bittencourt, et al. (2001). "Modeling panic attacks." Neuroscl Biobehav Rev 25(7B): 647-59.

Schmidt, N. B., J. K. Maner, et al. (2007). "Reactivity to challenge with carbon dioxide as a prospective predictor of panic attacks." Psychiatry Res.

Schmidt, N. B., J. H. Trakowski, et al. (1997). "Extinction of panicogenic effects of a $35 \% \mathrm{CO} 2$ challenge in patients with panic disorder." J Abnorm Psychol 106(4): 630-8.

Schruers, K., R. van Diest, et al. (2002). "Acute L-5-hydroxytryptophan administration inhibits carbon dioxide-induced panic in panic disorder patients." Psychiatry Res 113(3): 237-43. 


\section{Chapter 1}

Seddon, K., J. Potokar, et al. "The effects of a 7.5\% CO2 challenge in patients with Generalised Anxiety Disorder." J Psychopharmacol - in press.

Severson, C. A., W. Wang, et al. (2003). "Midbrain serotonergic neurons are central pH chemoreceptors." Nat Neurosci 6(11): 1139-40.

Shekhar, A., P. L. Johnson, et al. (2006). "Angiotensin-ll is a putative neurotransmitter in lactate-induced panic-like responses in rats with disruption of GABAergic inhibition in the dorsomedial hypothalamus." J Neurosci 26(36): 9205-15.

Shekhar, A. and S. R. Keim (1997). "The circumventricular organs form a potential neural pathway for lactate sensitivity: implications for panic disorder." J Neurosci 17 (24): 9726-35.

Shlik, J., A. Aluoja, et al, (1997). "Effects of citalopram treatment on behavioural, cardiovascular and neuroendocrine response to cholecystokinin tetrapeptide challenge in patients with panic disorder." J Psychiatry Neurosci 22(5): 332-40.

Siesjo, B. K. (1982). "Lactic acidosis in the brain: occurrence, triggering mechanisms and pathophysiological importance." Ciba Found Symp 87: 77-100.

Southwick, S. M., J. H. Krystal, et al. (1997). "Noradrenergic and serotonergic function in posttraumatic stress disorder." Arch Gen Psychiatry 54(8): 749-58.

Spinhoven, P., E. J. Onstein, et al. (1992). "The hyperventilation provocation test in panic disorder." Behav Res Ther 30(5): 453-61.

Strohle, A., C. Feller, et al. (2005). "The acute antipanic activity of aerobic exercise." Am J Psychiatry 162(12): 2376-8.

Strohle, A., F. Holsboer, et al. (2000). "Increased ACTH concentrations associated with cholecystokinin tetrapeptide-induced panic attacks in patients with panic disorder." Neuropsychopharmacology 22(3): $251-6$

Strohle, A., M. Kellner, et al. (1999). "Behavioral, neuroendocrine, and cardiovascular response to flumazenil: no evidence for an altered benzodiazepine receptor sensitivity in panic disorder." Biol Psychiatry 45(3): $321-6$.

Strohle, A., M. Kellner, et al. (1998). "Effect of flumazenil in lactate-sensitive patients with panic disorder." Am J Psychiatry 155(5): 610-2.

Strohle, A., E. Romeo, et al. (2003). "Induced panic attacks shift gamma-aminobutyric acid type A receptor modulatory neuroactive steroid composition in patients with panic disorder: preliminary results." Arch Gen Psychlatry 60(2): 161-8.

Swain, J., D. Koszycki, et al. (2003). Pharmacological challenge agents in anxiety. Anxiety dlsorders. D. J. Nutt and J. C. Ballenger. Malden, Mass., Blackwell Science: xi, $542 \mathrm{p}$.

Targum, S. D. and L. E. Marshall (1989). "Fenfluramine provocation of anxiety in patients with panic disorder." Psychiatry Res 28(3): 295-306.

Toru, I., J. Shlik, et al. (2006). "Tryptophan depletion does not modify response to CCK-4 challenge in patients with panic disorder after treatment with citalopram." Psychopharmacology (Berl) 186(1): 107-12.

Tsacopoulos, M. and P. J. Magistretti (1996). "Metabolic coupling between glia and neurons." $\underline{J}$ Neurosci $16(3): 877-85$.

Uhde, T. W. M. E. Tancer, et al. (1994). "Normal urinary free cortisol and postdexamethasone cortisol in social phobia: comparison to normal volunteers." J Affect Disord 30(3): 155-61.

Uhlenhuth, E. H. A. C. Leon, et al. (2006). "Psychopathology of panic attacks in panic disorder." $\rfloor$ Affect Disord $92(1): 55-62$.

Uhlenhuth, E. H., W. Matuzas, et al. (2000). "Do antidepressants selectively suppress spontaneous (unexpected) panic attacks? A replication." J Clin Psychopharmacol 20(6): 622-7.

Valenca, A. M., A. E. Nardi, et al. (2004). "Clonidine in respiratory panic disorder subtype." Arg Neuropsiquiatr 62(2B): 396-8.

Van den Hout, M. A. and E. Griez (1984). "Panic symptoms after inhalation of carbon dioxide." $\mathrm{Br} \mathrm{J}$ Psychiatry 144: 503-7.

van den Hout, M. A., G. M. van der Molen, et al. (1987). "Reduction of CO2-Induced anxiety in patients with panic attacks after repeated $\mathrm{CO} 2$ exposure." Am J Psychiatry 144(6): 788-91.

van Duinen, M. A., K. R. Schruers, et al. (2005). "CO2 challenge results in hypothalamic-pituitary-adrenal activation in healthy volunteers." J Psychopharmacol 19(3): 243-7. 
Introduction: Experimental models of panic

van Duinen, M. A., K. R. Schruers, et al. (2006). "CO2 challenge induced HPA axis activation in panic." int J Neuropsychopharmacol: 1-8.

van Megen, H. J., H. G. Westenberg, et al. (1994). "Pentagastrin induced panic attacks: enhanced sensitivity in panic disorder patients." Psychopharmacology (Berl) 114(3): 449-55.

van Megen, H. J., H. G. Westenberg, et al. (1997). "Effect of the selective serotonin reuptake inhibitor fluvoxamine on CCK-4 induced panic attacks." Psychopharmacology (Berl) 129(4): 357-64.

van Rijen, P. C., P. R. Luyten, et al. (1989). "1H and 31P NMR measurement of cerebral lactate, highenergy phosphate levels, and $\mathrm{pH}$ in humans during voluntary hyperventilation: associated EEG, capnographic, and Doppler findings." Magn Reson Med 10(2): 182-93.

van Vliet, I. M., H. G. Westenberg, et al. (1997). "Anxiogenic effects of pentagastrin in patients with social phobia and healthy controls." Biol Psychiatry 42(1): 76-8.

Vanderhaeghen, J. J., J. C. Signeau, et al. (1975). "New peptide in the vertebrate CNS reacting with antigastrin antibodies." Nature 257(5527): 604-5.

Veltman, D. J., G. A. van Zijderveld, et al. (1996). "Epinephrine infusions in panic disorder: a double-blind placebo-controlled study." $\rfloor$ Affect Disord 39(2): 133-40.

Verburg, K., E. Griez, et al. (1995). "Discrimination between panic disorder and generalized anxiety disorder by $35 \%$ carbon dioxide challenge." Am J Psychiatry 152(7): 1081-3.

Verburg, K., G. Perna, et al. (2001). A case study of the 35\% CO2 challenge. Anxiety disorders : an introduction to clinical management and research. E. J. L. Griez. Chichester ; New York, Wiley: xxii, $380 \mathrm{p}$.

Verburg, K., H. Pols, et al. (1998). "Reliability of the $35 \%$ carbon dioxide panic provocation challenge." Psychiatry Res 78(3): 207-14.

Vickers, K. and R. J. McNally (2005). "Respiratory symptoms and panic in the National Comorbidity Survey: a test of Klein's suffocation false alarm theory." Behav Res Ther 43(8): 1011-8.

Vorstrup, S., K. E. Jensen, et al. (1989). "Neuronal pH regulation: constant normal intracellular pH is maintained in brain during low extracellular $\mathrm{pH}$ induced by acetazolamide--31P NMR study." لـ Cereb Blood Flow Metab 9(3): 417-21.

Washburn, C. P., J. E. Sirois, et al. (2002). "Serotonergic raphe neurons express TASK channel transcripts and a TASK-like $\mathrm{pH}$ - and halothane-sensitive $\mathrm{K}+$ conductance." J Neurosci 22(4): 1256-65.

Wemmie, J. A., C. C. Askwith, et al. (2003). "Acid-sensing ion channel 1 is localized in brain regions with high synaptic density and contributes to fear conditioning." J Neurosci 23(13): 5496-502.

Wemmie, J. A., M. W. Coryell, et al. (2004). "Overexpression of acid-sensing ion channel $1 \mathrm{a}$ in transgenic mice increases acquired fear-related behavior." Proc Natl Acad Sci U SA 101(10): 3621-6.

Wiedemann, K., H. Jahn, et al. (2001). "Anxiolyticlike effects of atrial natriuretic peptide on cholecystokinin tetrapeptide-induced panic attacks: prellminary findings." Arch Gen Psychiatry 58(4): 371-7.

Withelm, F. H., A. L. Gerlach, et al. (2001). "Slow recovery from voluntary hyperventilation in panic disorder." Psychosom Med 63(4): 638-49.

Yeragani, V. K., R. Balon, et al. (1988). "Effects of laboratory-induced panic-anxiety on subsequent provocative infusions." Psychiatry Res 23(2): 161-6.

Yeragani, V. K., R. Pohl, et al. (1988). "Sodium lactate infusions after treatment with tricyclic antidepressants: behavioral and physiological findings." Blol Psychiatry 24(7): 767-74.

Zandbergen, J., H. H. Lousberg, et al. (1990). "Hypercarbia versus hypocarbia in panic disorder." J Affect Disord 18(2): 75-81.

Zandbergen, J., H. Pols, et al. (1991). "Ventilatory response to CO2 in panic disorder." Psychiatry Res $39(1): 13-9$.

Zhou, F. Q. (2005). "Pyruvate in the correction of intracellular acidosis: a metabolic basis as a novel superior buffer." Am J Nephrol 25(1): 55-63.

Zwanzger, P., T. C. Baghai, et al. (2001). "Vigabatrin decreases cholecystokinin-tetrapeptide (CCK-4) induced panic in healthy volunteers." Neuropsychopharmacology 25(5): 699-703.

Zwanzger, P., D. Eser, et al. (2003). "Effects of alprazolam on cholecystokinin-tetrapeptide-induced panic and hypothalamic-pituitary-adrenal-axis activity: a placebo-controlled study." Neuropsychopharmacology 28(5): 979-84.

Zwanzger, P., D. Eser, et al. (2003). "Effects of tiagabine on cholecystokinin-tetrapeptide (CCK-4)-induced anxiety in healthy volunteers." Depress Anxiety 18(3): 140-3. 
Chapter 1:

\title{
Part 2. The exercise-affect relationship
}

\author{
Esquivel $G^{1}$, Hartgens $F^{2}$, Schruers $K^{1}$ and Griez $E^{1}$
}

'Academic Anxiety Center and School for Mental Health and Neurosciences, Faculty of Medicine, Health and Life Sciences, Maastricht University, Maastricht, The Netherlands

${ }^{2}$ Department of Surgery, Outpatient Clinic Sports Medicine, University Hospital Maastricht, and Sports Medicine Center Maastricht, Maastricht,

The Netherlands

Published in part as: Esquivel, G., Hartgens F, K. Schruers and E. Griez (2008). "[Physical exercise as (adjuvant) treatment for depression and anxiety disorders]."

Stimulus 27(2): 90-107 
Introduction: The exercise affect relationship

\section{Summary}

That physical exercise can improve health is a centuries old empirical observation that has been confirmed only in the recent decades. The therapeutic and preventive effects of exercise for a wide range of physical conditions are well established. However, with regard to mental health, support has begun to accumulate for the benefits of exercise in depression and anxiety (Petruzzello, Landers et al. 1991; de Araujo, de Mello et al. 2007). These conditions are a worldwide prevalent cause of recurrent and lifelong psychosocial impairment and standard effective treatments are at best poorly used. Physical exercise has the potential to become a low-cost, stigma-free and readily available strategy to treat depression and anxiety.

Many observational studies have evaluated the association between levels of physical activity and measurements of mood and anxiety. Although most of these studies were not designed to specifically answer this question, they do provide a substantial insight into this relationship. Cross-sectional evidence of an inverse relationship between leisure-time physical activity and depressive symptoms seems unequivocal. However, not all results form prospective studies support this conclusion. Therefore caution must be taken when inferring a causal effect of physical activity over psychiatric complaints. Expectedly, the effects of exercise on symptoms of depression and anxiety in the general population are probably smaller than what cross-sectional analyses suggest and factors other than exercise may contribute to the positive effects.

There is a great amount of studies that test exercise as a therapeutic intervention for depression. Although most studies reveal a positive outcome for the effect of exercise, they also fail to meet the high quality requirements for clinical trials. Encouragingly, recent well-designed studies confirm the positive effects of exercise on depression. A similar scenario is found with regard to the results in anxiety. The evidence for the benefits of exercise in depression and anxiety is not unequivocal but the accumulated data is encouraging. The need for more exercise trials that are adequately designed is timely.

Many potential components can be thought as active ingredients when a patient feels better after exercise. As many social and cognitive aspects may contribute many recent trials have been designed to control for these factors. When it comes to the neurobiology behind the antidepressant and anti-anxiety effect of exercise, directions taken by some proposals focus on the brain circuitries dysfunctions established for these disorders. 
Introduction

The idea of a relationship between physical activity and wellbeing is deeply rooted in the history of human civilization (U.S. Dept. of Health and Human Services. 1996). In ancient Greece, aside from olympic champions being considered to have the strength of gods, early physicians such as Herodictus, Hippocrates and Galen had described the therapeutic and preventive use of physical activity. Present-day use of tai-chi-chuan and yoga represent an inheritance from the central role of physical activity in the ancient Chinese and Indian cultures. The centuries old empirical observation from that individuals with a sedentary lifestyle suffered from more ailments than those who were active has been confirmed only in the recent decades. The science behind the benefits of exercise and increased physical activity include a variety of disciplines such as physiology, epidemiology, psychology and medicine.

Evidence gathered from these fields is such that public health policy around the world has spent a great deal of effort to promote an increase in the level of physical activity in their communities. To this date, the therapeutic and preventive effects for a wide range of physical conditions such as cardiovascular disease, obesity, type 2 diabetes mellitus and cancer are well established (U.S. Dept. of Health and Human Services 1996).

With regard to mental health, support has began to accumulate for the benefits of exercise in depression (Lawlor and Hopker 2001) and anxiety (Petruzzello, Landers et al. 1991; de Araujo, de Mello et al. 2007). Additionally, exercise may improve cognitive function (Etnier, Nowell et al. 2006), and has been use to treat substance use disorders (Daniel, Cropley et al. 2006) and obsessive-compulsive disorder (Brown, Abrantes et al. 2007). Depression and anxiety disorders are an estimated burden of 146,000 million euros to European society (Andlin-Sobocki, Jonsson et al. 2005) and by the year 2010 depression alone is expected to be the second most disabling human disorder (Ustun, Ayuso-Mateos et al. 2004). These conditions are a worldwide prevalent cause of recurrent and lifelong psychosocial impairment and standard effective treatments are at best poorly used (Johnson 1981). Therefore, the development of alternative strategies that can prolong remission, improve the patient's quality of life and reduce the enormous burden that these disorders impose on the community is timely. Physical exercise has the potential to become a low-cost, stigma-free and readily available strategy to treat depression and anxiety.

This paper has the objective to provide an overview of the relationship between physical activity and depression and anxiety. A selection of epidemiological cross-sectional surveys and cohort studies, along with exercise experimental trials will be abridged before a discussion regarding the evidence for the validity of this knowledge. In addition, some of the biological mechanisms regarded as explanations for the effects of exercise on depression and anxiety will be mentioned. Finally, we will summarize some of the particular aspects to take 
Introduction: The exercise affect relationship

into account when incorporating exercise as treatment for patients that suffer these conditions.

The relationship between physical activity and mental health

Several cross-sectional and longitudinal studies have evaluated the association between levels of physical activity and measurements of mood and anxiety (Farmer, Locke et al. 1988; Stephens 1988; Weyerer 1992; Cooper-Patrick, Ford et al. 1997; Kritz-Silverstein, BarrettConnor et al. 2001; Sexton, Sogaard et al. 2001; Goodwin 2003; Simon, Powell et al. 2004; Kaiya, Umekage et al. 2005; De Moor, Beem et al. 2006; Galper, Trivedi et al. 2006; Ohta, Mizoue et al. 2007; Wiles, Haase et al. 2007). Although these studies have provided a substantial insight into this relationship, unfortunately none has met the ideal requirements to draw a satisfactory epidemiological conclusion. Most results come from large epidemiological studies that were designed to answer many other public health questions, subsequently, measurements of physical activity or fitness were rather crude and consisted of one or a few questions regarding participation in exercise. Moreover, as most studies only considered leisure-time physical activity, total physical activity is underestimated. Another reservation to be taken into account is that some of the data stems from samples not representative of the general population. Finally, the difficulty to determine the direction of causality is further hindered by the lack of analysis regarding the influence baseline anxiety or depression could have on exercise and physical activity participation in prospective studies (reverse causality).

Most cross-sectional studies that describe the relationship between physical activity and depression analyze data obtained from large non-institutionalized population samples taken from different parts of the developed world (Stephens 1988; Goodwin 2003; De Moor, Beem et al. 2006; Ohta, Mizoue et al. 2007). Measurements of physical activity vary from single questions to comprehensive questionnaires that include work-related and leisure time physical activities. Psychiatric symptoms were usually determined with epidemiological questionnaires that give an index of mood and anxiety. In the sample presented by Goodwin (2003), psychiatric diagnosis were determined with the comprehensive diagnostic interview where the presence or absence of several of several common disorders, including panic and depression, were evaluated. The results of these studies are consistent with a positive association between levels of physical activity with general well-being, positive mood, lower levels/incidence of depression and anxiety. With regard to panic, a Japanese survey of 4000 adults revealed the association of dislike of exercise with panic attacks and panic disorder (Kaiya, Umekage et al. 2005).

One cross-sectional study was performed using data from the Aerobics Center Longitudinal Study (Galper, Trivedi et al. 2006). Here, the physical activity rating and 
cardio-respiratory fitness determined with a maximal treadmill test of 5451 men and 1277 woman was inversely related in a dose-dependant manner with depressive symptoms. This constitutes the first study to relate well-validated measures of physical activity with depressive symptoms. The main limitation of this study is that the population sample is representative of white Americans with better than average socioeconomic status.

Many other studies obtained data on physical activity and symptoms of depression and anxiety on additional occasions over time and were able to make both cross-sectional and longitudinal analyses (Farmer, Locke et al. 1988; Weyerer 1992; Kritz-Silverstein, BarrettConnor et al. 2001; Sexton, Sogaard et al. 2001; Wiles, Haase et al. 2007). These studies consistently show a cross-sectional relationship between measurements of physical activity and fewer symptoms of depression and anxiety. Although some prospective research lends support to these findings (Farmer, Locke et al. 1988; Wiles, Haase et al. 2007), other studies do not find a longitudinal relationship between these symptoms and physical activity Weyerer 1992; Cooper-Patrick, Ford et al. 1997; Kritz-Silverstein, Barrett-Connor et al. 2001; Sexton, Sogaard et al. 2001). One study evaluated the prospective association between levels of physical activity and depression in 424 clinically depressed patients in 4 waves over 10 years (Harris, Cronkite et al. 2006). Higher physical activity was related to less concomitant depression over the 4 measurements. Moreover, these authors found no evidence of reverse causation; in other words, baseline depression did not influence the trajectory of physical activity,

Taken all together, the cross-sectional evidence of an inverse relationship between leisure-time physical activity and depressive symptoms seems unequivocal. However, results form prospective studies suggest caution when inferring a causal effect of physical activity over psychiatric complaints. The lack of uniform results across studies can be accounted by the differences in methods to determine activity and psychiatric assessments as well as population sizes and compositions. Expectedly, the effects of exercise on symptoms of depression (and perhaps anxiety) in the general population are probably smaller than what cross-sectional analyses suggest and factors other than exercise may contribute to the positive effects.

The use of physical exercise as treatment in clinical populations

Due to the nature of depressive and anxious disorders, all experimental studies using exercise as treatment have focused on mild to moderate cases. Furthermore, trials can only include those subjects willing to engage in exercise and usually exclude those already involved in regular physical activity. Notwithstanding, group underrepresentation is a limitation of any type of treatment trial, as many depressed patients will refuse pharmacological, psychological or 
Introduction: The exercise affect relationship

any treatment altogether. Another problem faced by exercise and other complex intervention trials is the difficulty to eliminate the potential bias from the subject's expectations and preferences by proper blinding. A proposed solution for future studies is compare exercise to a standard form of treatment and randomize only those individuals with no preference (Campbell, Fitzpatrick et al. 2000).

\section{In depression}

Depression is a common psychiatric disorder with a lifetime prevalence of about $15 \%$. The key symptoms of affected subjects is a constant and persistent feeling of sadness and/or a loss of interest or pleasure that last for at least two weeks. Additionally they will experience a number other complaints such as low energy, a reduction or increase of appetite, difficulty sleeping and concentrating, agitation or slow thoughts and movements, feeling of worthlessness, excessive guilt and thought of death or even suicide among many other symptoms. Depending on the severity of the episode, this condition can be very debilitating and cause a great amount of impairment on the individuals functioning.

Various systematic reviews can give account for outcome of the most important studies that test exercise as a therapeutic intervention for depression (North, McCullagh et al. 1990; Craft and Landers 1998; Lawlor and Hopker 2001). The most recent of these studies pooled the effects of 9 randomized controlled trials that compared exercise with no treatment and 4 studies that compared exercise with cognitive therapy (Lawlor and Hopker 2001). The results suggest that the therapeutic effect of exercise reported by those trials published in peer-reviewed articles is comparable-if not superior-to that of standard antidepressant treatment (Arroll, Macgillivray et al. 2005). Additionally, it appears that both aerobic and anaerobic exercises are effective. Although the results of this systematic review are encouraging, authors suggest that these results are to be taken with caution because of the poor quality of most studies. For instance, treatment allocation may have been properly concealed only in 3 studies and intention-to-treat analysis was undertaken in only 2. Another issue is that the main outcome measures in most studies were the subjects themselves, whereas in the only two studies with main outcome determined by rater, only one was blind.

Dunn and collaborators (2005), in recent well-designed study, randomized depressed subjects to either of two doses of exercise or a stretching exercise control condition for 12 weeks. Only doses of exercise consistent with recommendations to increase cardiovascular fitness (American College of Sports Medicine., Whaley et al. 2006) was an effective treatment for depression. This tightly controlled trial effectively concealed treatment allocation, used blind raters and minimized the effects of socialization by training subjects in individual rooms monitored by laboratory staff. An important drawback, as with all studies that test exercise as a therapeutic intervention, subjects were not blinded to treatment allocation where drop-out 


\section{Chapter 1}

were greater in the control condition. Set aside these limitations, this study an important piece of evidence that gives further support to the use of exercise as therapy for depression.

In anxiety

Anxiety, as loosely defined, is a normal defensive response to danger. This defensive reaction can protect us from a wide range of environmental threats such as an approaching motor vehicle or the social consequences of arriving late to work. More specifically, anxiety is distinguished from fear and panic attacks. In a healthy state, anticipatory anxiety is reaction to a threat that can be distant, vague, internal and conflictive in nature as it is usually approached-for instance getting married. Fear, on the other hand, is a reaction to a near, imminent and non-conflicting danger where avoidance behavior is most suitable-running away from a threatening animal. Panic attacks are brief unexpected spells of intense distress usually accompanied by several physical symptoms such as palpitations and breathlessness. These attacks are not considered part of a normal defensive repertoire, however their benign presence in healthy individuals is not uncommon. In general, these reactions are usually accompanied by peripheral manifestations such as palpitations, perspiration, dizziness, light-headiness, diarrhea, hypertension, restlessness, abdominal complaints, shivering and paresthesias. Subjects with anxiety disorders usually present a combination of anxiety, fear and panic attacks in a frequency and intensity that is usually unwarranted. Additionally, in affected individuals these reactions can reach such an extent that learning, thinking and perceptions can be reduced or distorted. Uitimately, that distinguishes normal reactions from pathological anxiety is a significant interference with daily occupational and social functioning.

In the case of panic disorder and agoraphobia, spontaneous panic attacks and the anxiety produced by concerns of new attacks or their consequences are the core clinical picture. Social and specific phobias are characterized by fear and anxiety mainly restricted to the possibility of social scrutiny and a specific situation (usually of some evolutionary significance such as spiders or heights) respectively. When individuals experience severe symptoms of fear and anxiety due the persistent recall of a traumatic experience the diagnosis of acute stress disorder or post-traumatic stress disorder may be warranted. In obsessive-compulsive disorder, the characteristic intrusive, recurrent and disturbing ideas, impulses or images are endured with much anxiety. Those affected with generalized anxiety disorder are troubled with persistent and uncontrollable worries of just about everything. As a rule, most of these conditions are comorbid with another anxiety disorder and depression.

Petruzzello (1991) presents an extensive review and meta-analysis of the effects sizes drawn from a large pool of published and unpublished exercise trials designed to reduce anxiety. State, trait and physiological measures of anxiety were separately analyzed along with potential moderating variables divided in exercise, study design, author and publication type 
Introduction: The exercise affect relationship

characteristics. Overall their findings support an anti-anxiety effect for exercise. Among other specific findings, changes in trait anxiety were related to programs that lasted more than 10 weeks and larger effects were surprisingly found in studies with randomized allocation of treatment (non-randomized studies are thought to have inflated effect-sizes). An important limitation of this review, with regard to the focus of this paper, is that it hardly includes any properly designed trial on the effects of exercise training in a well-defined clinical population. Additionally, no data was provided on the handling of withdrawals or other qualitative aspects of the studies reviewed. A recent systematic review on the effects of exercise on anxiety disorders gives account for the fact that very few studies have been published using clinical populations and are mainly restricted to panic disorder (de Araujo, de Mello et al. 2007). With regard for acute or single sessions of exercise, intense physical activity may increase anxiety (Rief and Hermanutz 1996) but does not provoke panic attacks in patients with panic disorder (Stein, Papp et al. 1992; Martinsen, Raglin et al. 1998). Our group has shown that moderate/hard exercise reduces the susceptibility to panic with inhaled $\mathrm{CO} 2$, a safe and validated model to recreate panic symptoms, in patients with panic disorder (Esquivel, DiázGalvis et al. 2007). To our knowledge only one well designed study has tested the therapeutic effects of exercise in panic disorder (Broocks, Bandelow et al. 1998). This study has shown that an aerobic exercise program has a therapeutic effect comparable to standard treatment with anti-panic medication. This last work however, could not disentangle the effects of exposure, socialization and found no relationship between endurance capacity and symptom severity (Meyer, Broocks et al. 1998). As such, the specificity of aerobic exercise as treatment for panic disorder remains undetermined.

\section{The level of evidence}

Can depressed and anxious patients really alleviate their symptoms with exercise? One common aid to answer this question is the use of a one of many schemes for the ranking of researched-based clinical knowledge. A major debate with regard to the usefulness of these methods of classifying evidence is that they focus almost entirely on study design with almost no regard to methodological appropriateness (Petticrew and Roberts 2003). In a strict sense, an exercise trial would have great difficulty to meet all the requirements of a good randomized controlled trial as it is impossible to incorporate an adequate placebo condition and blind participants. Additional to this unavoidable limitation, it is unfortunate that most exercise clinical trial in depression so far have been inadequately randomized (Lawlor and Hopker 2001) which makes conclusions difficult to draw since this limitation is thought to inflate effect sizes (Schulz, Chalmers et al. 1995). Callaghan (2004) has presented a further analysis where even conservatively reducing the average effect size of a recent meta-analysis to account of the lack 
of appropriate randomization, exercise could still potentially explain a 0.66 standard deviation reduction in depression scores. This "corrected" effect size is substantial compared to that of standard anti-depressive therapy with anti-depressive medication (Walsh, Seidman et al. 2002). The need for more exercise trials that are adequately designed is timely; and if the results of observational and experimental studies are taken together, this task is encouraging.

\section{The potential mechanisms of action}

When we imagine a patient feeling better after integrating in an exercise group conducted by a skilled and reassuring professional, many potential components can be thought as active ingredients. For instance, socialization is known to have positive effects in many mental health conditions. Simple supportive interventions are effective psychosocial tools that are contingent to the skills and attitudes of their providers. To be able to apply the knowledge and findings produced by research is necessary to determine the key active components in delivering successful exercise therapy. In this scope, recent trials have been designed to control for socialization and supportive interventions by staff (Dunn, Trivedi et al. 2002; Dunn, Trivedi et al. 2005) and determine if whether exercise has a dose-response effect (Dunn, Trivedi et al. 2001). Many mechanisms have been proposed to explain the effect of exercise on mental health. Here we will mention some examples of biological explanations that we consider representative.

Compared to different aspects of exercise physiology, very little is know of its neurobiology. For instance, in the scope of prevention and treatment of mental and neurological conditions, there is much to be leaned about the immediate and long term effects on the CNS produced by the different types of exercise, the duration and intensity of single bouts and the number and frequency of sessions (Dishman, Berthoud et al. 2006). A direction taken by some proposals regarding the neurobiology behind the antidepressant and anti-anxiety effect of exercise is one that focuses on the brain circuitries dysfunctions established for these disorders (e.g. hormone responses to stress adaptation and serotonin neurotransmitter function).

The changes in response to stress and specific serotonergic (e.l. serotonin, a ubiquitous neurotransmitter involved the homeostasis of several brain functions including mood and anxiety) brain activity in animal models after exercise (Dishman, Berthoud et al. 2006) is consonant with current views concerning the mechanisms of antidepressive and anxiolytic treatment (Nutt 2002; Strohle and Holsboer 2003; Graeff 2004). In humans, neurohumoral and pharmacological responses to 10 weeks of aerobic training in healthy subjects (Broocks, Meyer et al. 2001) and PD patients (Broocks, Meyer et al. 2003), respectively, suggest a modulation a $5-\mathrm{HT} 1 \mathrm{~A}$ and $5-\mathrm{HT} 2 \mathrm{C}$ receptors that could be linked to 
Introduction: The exercise affect relationship

mechanisms involved in the quiescence of spontaneous panic attacks. The implication of the endogenous opioid system in the beneficial effects is both popular and controversial (Salmon 2001). Although exercise is related to increased peripheral and central opioid activity (Sforzo 1989), the link between these events and psychological outcome is not without inconsistencies and may constitute an oversimplification (Dietrich and McDaniel 2004). Nonetheless, the results of controlled studies where pre-medication with the opioid receptor blocker naltrexone eliminates the positive effects of exercise on mood and anxiety are encouraging (Daniel, Martin et al. 1992; Jarvekulg and Viru 2002).

Issues on the implementation of physical exercise in patients with depression and anxiety

\section{Exercise referral and patient selection}

The popular and widespread use of exercise can be such that individuals with depression and anxiety may successfully initiate exercise on their own initiative or by the recommendation from peers or health providers. Unfortunately, this is probably an unlikely scenario given the number of barriers to behavior change inherent to the clinical picture of depressive and anxious symptoms (e.g. low energy levels, apathy, avoidance). As patients with these symptoms will require help with motivation, programming and behavior change, an appropriate scheme may be the direct referral by a competent clinician to a qualified exercise professional. Such a model of exercise implementation may raise issues regarding professional liability, the general quality of the service provided and the ethical aspects of exchanging details of patient's medical history and other sensitive information (Department of Health 2001).

The careful selection of patients for whom exercise therapy may be appropriate is necessary for several reasons. First of all, any individual with symptoms of clinical depression or anxiety should be evaluated by a competent health professional before any type of therapy is initiated to combat these conditions. The delay of a warranted clinical evaluation can have detrimental consequences that could have otherwise been prevented. Evidence so far supports the use of exercise in patients with anxiety and depression of mild to moderate severity. Accordingly, the exercise professional should also be alert for patients whose mental health condition can worsen to a point that exercise may no longer be suitable and provide feed back to other health care providers involved. Evidently, an open channel of communication between the exercise professional and the general practitioner or other care providers is necessary to seek advice when needed.

\section{Exercise dosage and modality}

The fashion in which exercise is delivered in most studies reflects the influence of the research in cardiovascular fitness. Hence, the bulk of evidence lies with 3 to 5 weekly sessions of 
Chapter 1

exercise involving large muscular groups at intensity necessary to reach about 60 to $70 \%$ of the individual's maximum oxygen consumption NO2max) for at least 10 weeks (Petruzzello, Landers et al. 1991; Lawlor and Hopker 2001). In the report of a carefully controlled study, Dunn and colleagues (2005) suggest that aerobic exercise below the threshold of public health recommendations (American College of Sports Medicine., Whaley et al. 2006) has an effect comparable to placebo in the treatment of depression. With regard to anaerobic exercise, some studies suggest this modality may be equally effective (Martinsen, Hoffart et al. 1989; Lawlor and Hopker 2001), nevertheless there is still need for carefully controlled studies to draw a recommendation.

As many patients with depression and anxiety may feel too uncomfortable with the level of effort and symptoms provoked by exercise, the initial doses of exercise may have to be lower than with the typical sedentary client. During the initial phases, some authors advocate longer but less intense workouts allowing subjects to take as many breaks (such as walking) as needed to ensure compliance (Meyer and Broocks 2000).

Studies that attempt to test exercise as an anti-depressive or anti-anxiety maneuvers will usually try to control for all other potential sources of beneficial effects, such as socialization, group sessions, behavioral-cognitive and supportive interventions, outdoor exercise and team sports. However, in a clinical setting attempts should be made to use all available resources to benefit clients. For instance patients with depression and social phobia may additionally benefit from exercising in groups and team sports. In patients with panic disorder small bouts of intense exercise can serve as exposure to internal cues and physical sensations, which these patients typically fear. Similarly, outdoor exercise in subjects with agoraphobia (fear of open spaces) can be used as exposure to fearful scenarios. In just about any patient, the use of cognitive-behavioral techniques to address issues related with the clients symptoms and their barriers to behavior change and compliance will be beneficial.

The exercise professional should additionally keep in mind the special characteristics and needs of patients with depression and anxiety. For instance anxious patients may be less tolerant to the bodily sensations provoked by the increased physical activity where a reassuring attitude from the trainer may prove helpful. Depressed patients can display little motivation, low energy levels and difficulty concentrating. In this way, close monitoring, frequent encouragement and more time for instructions to be comprehended may be necessary.

\section{Documenting output: physical and psychological gains}

Most exercise professionals are well versed in the documentation of physical gains. The reasons for doing so are quite straightforward. For instance, an initial evaluation of body mass index, aerobic endurance, physical limitations, exercise preferences and therapy expectations 
Introduction: The exercise affect relationship

are necessary to draw an adequate treatment plan for a given client. Sophisticated equipment is not necessary for a basic initial evaluation. Although the maximal spiroergometry test is the standard, an adequate VO2max estimation can be done using one of many standardized procedures for bicycle or treadmill ergometers or a step test (American College of Sports Medicine., Whaley et al. 2006). As many individuals with anxiety or depression protocol may have difficulties reaching their physical exertion limits, some exercise laboratories experienced with patients suffering from depression and anxiety disorders recommend submaximal tests when precision is not an issue and blood lactate responses to incremental workloads as a more reliable option (Meyer and Broocks 2000).

The monitoring of training can be easily kept with the use portable heart rate devices now widely available or with the use of the rating of perceived exertion scale (RPE) where subjects can rate their subjective exertion on a simple visual list (See: www.cdc.gov). Monitoring is important not only to ensure a proper dose of exercise, but also to provide the client with means of self-monitoring that will be key in organizing long-term home-based training.

Recording the changes in depressive an anxious symptoms can be valuable. Positive feedback on the reinforcement of exercise behavior is a well established strategy (American College of Sports Medicine., Whaley et al. 2006). Set aside the changes in physical aspects (e.g. cardiovascular fitness, weight) the documentation of improvement in mood and reductions of anxiety can be powerful incentives for patients to continue exercising. Ideally exercise programs should be provided as an integrated mental health service where most team members will be well versed in specific symptoms and global improvement rating. In any case, simple notes of the impression of improvement by the exercise professional and the patient can be used for purpose of providing feedback.

\section{Conclusions}

Exercise is a promising form of therapy for anxiety and depression. This is of major importance given that these conditions are a worldwide prevalent cause of human dysfunction where alternative forms of treatment are needed. Although unequivocal support for its effectiveness is pending, evidence for the effects of exercise in these patients is accumulating. Parallel to the need for carefully designed studies, an adequate framework to evaluate the evidence of exercise and other forms of complex interventions is pending.

Many biological and psychological mechanisms for the effects of exercise in mood and anxiety have been set forward. The much-needed efforts in this direction will not only help to improve the implementation of effective exercise therapy but can also provide insight into the underlying processes of anxiety and depression and its treatment. 


\section{Chapter 1}

The special needs and characteristics of patients with depression and anxiety may need to be considered when implementing exercise. These aspects are important for the evaluation and practical aspects of training as well as the selection, depending on the patient's symptoms, of certain exercise modalities that can be tailored to maximize potential benefits. 
Introduction: The exercise affect relationship

\section{References}

American College of Sports Medicine., M. H. Whaley, et al. (2006). ACSM's quidelines for exercise testing and prescription. Philadelphia, Lippincott Williams \& Wilkins.

Andlin-Sobocki, P., B. Jonsson, et al. (2005). "Cost of disorders of the brain in Europe." Eur J Neurol 12 Suppl 1: 1-27.

Arroll, B., S. Macgillivray, et al. (2005). "Efficacy and tolerability of tricyclic antidepressants and SSRIs compared with placebo for treatment of depression in primary care: a meta-analysis. " Ann Fam Med 3(5): 449-56.

Broocks, A., B. Bandelow, et al. (1998). "Comparison of aerobic exercise, clomipramine, and placebo in the treatment of panic disorder." Am J Psychiatry 155(5): 603-9.

Broocks, A., T. Meyer, et al. (2001). "Effect of aerobic exercise on behavioral and neuroendocrine responses to meta-chlorophenylpiperazine and to ipsapirone in untrained healthy subjects." Psychopharmacology (Berl) 155(3): 234-41.

Broocks, A., T. Meyer, et al. (2003). "5-HT1A responsivity in patients with panic disorder before and after treatment with aerobic exercise, clomipramine or placebo." Eur Neuropsychopharmacol 13(3): 153-64.

Brown, R. A., A. M. Abrantes, et al. (2007). "A pilot study of moderate-Intensity aerobic exercise for obsessive compulsive disorder." J Nerv Ment Dis 195(6): 514-20.

Callaghan, P. (2004). "Exercise: a neglected intervention in mental health care?" J Psychiatr Ment Health Nurs 11(4): 476-83.

Campbell, M., R. Fitzpatrick, et al. (2000). "Framework for design and evaluation of complex interventions to improve health." Bmi 321 (7262): 694-6.

Cooper-Patrick, L., D. E. Ford, et al. (1997). "Exercise and depression in midlife: a prospective study." Am J Public Health 87(4): 670-3.

Craft, L. L. and D. M. Landers (1998). "The effect of exercise on clinical depression and depression resulting from mental illness: a meta-analysis." J Sport Exerc Psychol 20(4): 339-57.

Daniel, J. Z., M. Cropley, et al. (2006). "The effect of exercise in reducing desire to smoke and cigarette withdrawal symptoms is not caused by distraction." Addiction 101(8): 1187-92.

Daniel, M., A. D. Martin, et al. (1992). "Opiate receptor blockade by naltrexone and mood state after acute physical activity." Br J Sports Med 26(2): 111-5.

de Araujo, S. R., M. T. de Mello, et al. (2007). "[Anxlety disorders and physical exercise]." Rev Bras Psiquiatr 29(2): 164-71.

De Moor, M. H., A. L. Beem, et al. (2006). "Regular exercise, anxiety, depression and personality: a population-based study." Prev Med 42(4): 273-9.

Department of Health (2001). Exercise Referral Systems: A National Quality Assurance Framework. London, UK., Department of Health.

Dietrich, A. and W. F. McDaniel (2004). "Endocannabinoids and exercise." Br J Sports Med 38(5): 536-41.

Dishman, R. K., H. R. Berthoud, et al. (2006). "Neurobiology of exercise." Obesity (Silver Spring) 14(3): 345-56.

Dunn, A. L., M. H. Trivedi, et al. (2002). "The DOSE study: a clinical trial to examine efficacy and dose response of exercise as treatment for depression." Control Clin Trials 23(5): 584-603.

Dunn, A. L., M. H. Trivedi, et al. (2005). "Exercise treatment for depression: efficacy and dose response." Am J Prev Med 28(1): $1-8$.

Dunn, A. L., M. H. Trivedi, et al. (2001). "Physical activity dose-response effects on outcomes of depression and anxiety." Med Sci Sports Exerc 33 (6 Suppl): S587-97; discussion 609-10.

Esquivel, G., J. Diáz-Galvis, et al. (2007). "Acute exercise reduces the effects of a 35\% CO2 challenge in patients with panic disorder." Journal of affective Disorders (In print).

Etnier, J. L., P. M. Nowell, et al. (2006). "A meta-regression to examine the relationship between aerobic fitness and cognitive performance." Brain Res Rev 52(1): 119-30.

Farmer, M. E., B. Z. Locke, et al. (1988). "Physical activity and depressive symptoms: the NHANES I Epidemiologic Follow-up Study." Am J Epidemiol 128(6): 1340-51. 
Chapter 1

Galper, D. I., M. H. Trivedi, et al. (2006). "Inverse association between physical inactivity and mental health in men and women." Med Sci Sports Exerc 38(1): 173-8.

Goodwin, R. D. (2003). "Association between physical activity and mental disorders among adults in the United States." Prev Med 36(6): 698-703.

Graeff, F. G. (2004). "Serotonin, the periaqueductal gray and panic." Neurosci Biobehav Rev 28(3): 23959.

Harris, A. H., R. Cronkite, et al. (2006). "Physical activity, exercise coping, and depression in a 10-year cohort study of depressed patients." J Affect Disord 93(1-3): 79-85.

Jarvekulg, A. and A. Viru (2002). "Opioid receptor blockade eliminates mood effects of aerobic gymnastics." Int J Sports Med 23(3): 155-7.

Johnson, D. A. (1981). "Depression: treatment compliance in general practice." Acta Psychiatr Scand Suppl 290: 447-53.

Kaiya, H. T. Umekage, et al. (2005). "Factors associated with the development of panic attack and panic disorder: survey in the Japanese population." Psychiatry Clin Neurosci 59(2): 177-82.

Kritz-Silverstein, D., E. Barrett-Connor, et al. (2001). "Cross-sectional and prospective study of exercise and depressed mood in the elderly : the Rancho Bernardo study." Am J Epidemiol 153(6): 596603.

Lawlor, D. A. and S. W. Hopker (2001). "The effectiveness of exercise as an intervention in the management of depression: systematic review and meta-regression analysis of randomised controlled trials." Bmi 322(7289): 763-7.

Martinsen, E. W., A. Hoffart, et al. (1989). "Comparing aerobic with nonaerobic forms of exercise in the treatment of clinical depression: a randomized trial." Compr Psychiatry 30(4): 324-31.

Martinsen, E. W., J. S. Raglin, et al. (1998). "Tolerance to intensive exercise and high levels of lactate in panic disorder." J Anxiety Disord 12(4): 333-42.

Meyer, T. and A. Broocks (2000). "Therapeutlc impact of exercise on psychiatric diseases: guidelines for exercise testing and prescription." Sports Med 30(4): 269-79.

Meyer, T., A. Broocks, et al. (1998). "Endurance training in panic patients: spiroergometric and clinical effects." Int J Sports Med 19(7): 496-502.

North, T. C., P. McCullagh, et al. (1990). "Effect of exercise on depression." Exerc Sport Sci Rev 18: 379415.

Nutt, D. J. (2002). "The neuropharmacology of serotonin and noradrenaline in depression." Int Clin Psychopharmacol 17 Suppl 1:S1-12.

Ohta, M., T. Mizoue, et al. (2007). "Effect of the physical activities in leisure time and commuting to work on mental health." J Occup Health 49(1): 46-52.

Petruzzello, S. J., D. M. Landers, et al. (1991). "A meta-analysis on the anxiety-reducing effects of acute and chronic exercise. Outcomes and mechanisms." Sports Med 11(3): 143-82.

Petticrew, M. and H. Roberts (2003). "Evidence, hierarchies, and typologies: horses for courses." $\underline{J}$ Epidemiol Community Health 57(7): 527-9.

Rief, W. and M. Hermanutz (1996). "Responses to activation and rest in patients with panic disorder and major depression." Br J Clin Psychol 35 ( Pt 4): 605-16.

Salmon, P. (2001). "Effects of physical exercise on anxiety, depression, and sensitivity to stress: a unifying theory." "Clin Psychol Rev 21(1): 33-61.

Schulz, K. F., I. Chalmers, et al. (1995). "Empirical evidence of bias. Dimensions of methodological quality associated with estimates of treatment effects in controlled trials." Jama 273(5): 408-12.

Sexton, H., A. J. Sogaard, et al. (2001). "How are mood and exercise related? Results from the Finnmark study." Soc Psychiatry Psychiatr Epidemiol 36(7): 348-53.

Sforzo, G. A. (1989). "Opioids and exercise. An update." Sports Med 7(2): 109-24.

Simon, T. R. K. E. Powell, et al. (2004). "Involvement in physical activity and risk for nearly lethal suicide attempts." Am J Prev Med 27(4): 310-5.

Stein, J. M., L. A. Papp, et al. (1992). "Exercise tolerance in panic disorder patients." Biol Psychiatry 32(3): $281-7$.

Stephens, T. (1988). "Physical activity and mental health in the United States and Canada: evidence from four population surveys." Prev Med 17(1): 35-47. 
Introduction: The exercise affect relationship

Strohle, A. and F. Holsboer (2003). "Stress responsive neurohormones in depression and anxiety." Pharmacopsychiatry 36 Suppl 3: S207-14.

U.S. Dept. of Health and Human Services (1996). Physical activity and health: a report of the Surgeon General. Attanta, GA., U.S. Dept. of Health and Human Services, Centers for Disease Control and Prevention.

U.S. Dept. of Health and Human Services. (1996). Physical activity and health : a report of the Surgeon General. Atlanta, GA., U.S. Dept. of Health and Human Services, Centers for Disease Control and Prevention.

Ustun, T. B., J. L. Ayuso-Mateos, et al. (2004). "Global burden of depressive disorders in the year 2000." Br J Psychiatry 184: 386-92.

Walsh, B. T., S. N. Seidman, et al. (2002). "Placebo response in studies of major depression: variable, substantial, and growing." Jama 287(14): 1840-7.

Weyerer, S. (1992). "Physical inactivity and depression in the community. Evidence from the Upper Bavarian Field Study." Int J Sports Med 13(6): 492-6.

Wiles, N. J., A. M. Haase, et al. (2007). "Physical activity and common mental disorder; results from the Caerphilly study." Am J Epidemiol 165(8): 946-54. 
Chapter 2:

\title{
The effects of acute exercise on $35 \% \mathrm{CO} 2$ induced panic in healthy volunteers
}

\author{
Esquivel $G^{1}$, Schruers $K^{1}$, Kuipers $H^{2}$ and Griez $E^{1}$ \\ 'Department of Psychiatry and Neuropsychology and ${ }^{2}$ Department of \\ Movement Sciences, Faculty of Medicine, Health and Life Sciences, \\ Maastricht University
}

Published as: Esquivel, G., K. Schruers, H. Kuipers and E. Griez (2002). "The effects of acute exercise and high lactate levels on 35\% CO2 challenge in healthy volunteers."

Acta Psychiatr Scand 106(5): 394-7 
The effects of acute exercise on $35 \% \mathrm{CO} 2$ induced panic in healthy volunteers

\begin{abstract}
Objective: To test the possible antipanic effects of acute exercise in healthy volunteers exposed to an inhalation of $35 \% \mathrm{CO} 2$ challenge.

Method: Twenty healthy subjects in a randomized separate group design, performed exercise in a bicycle ergometer reaching $>6 \mathrm{~mm}$ of blood lactate and a control condition of minimal activity in the same fashion with no lactate elevation. Immediately afterwards an inhalation of a vital capacity using a mixture of $35 \% \mathrm{CO} 2 / 65 \%$ O2 through a mask was given on both conditions.

Results: Subjects under the exercise condition reported less panic symptoms than controls after a CO2 challenge on the diagnostic statistical manual-IV (DSM-IV) Panic Symptom List but no difference on the Visual Analogue Anxiety Scale.

Conclusion: Subjects under the exertion condition had lactate levels comparable with those of lactate infusions but an inhibitory rather than accumulative effect was seen when combined with a CO2 challenge.
\end{abstract}

Key words: carbon dioxide, exertion, lactic acid, panic disorder. 


\section{Chapter 2}

Introduction

Carbon dioxide and lactate have proven to reliably induce panic in patients with PD. Liebowitz (1984) and collaborators at the Columbia University infused patients meeting DSM-III criteria for panic disorder or agoraphobia with panic attacks with $0.5 \mathrm{M}$ racemic sodium lactate before and after successful drug treatment. They found that lactate-induced and naturally occurring panic attacks were symptomatically similar and following treatment, the patients' response to lactate did not differ from that of normal controls. Griez (1990) and collaborators applied one inhalation of $35 \% \mathrm{CO} 2$ and compressed air as placebo control to 36 patients with anxiety disorders (18 with diagnosis of PD and 18 of obsessive-compulsive disorder [OCD]) and 14 healthy controls in a double-blind design. Carbon dioxide provoked high levels of subjective anxiety in the PD group while patients with OCD were hardly affected by the inhalation. This same team designed a test-retest reliability of the 35\% CO2 panic provocation challenge with 30 patients with PD Nerburg, Pols et al. 1998). Twenty-four (Six patients dropped out) were challenged twice with 1 week between the two challenges and showed a good test-retest reliability; on both induced subjective anxiety, measured on the VAS and induced panic symptoms, measured with a PSL. This study also concluded that assessing the state of anxiety immediately after the challenge gave the most reliable results.

Exercise in these patients can produce lactate elevations at a level and rate twice as greater than during lactate infusion without producing panic. Stein (1992) published a study with 16 patients with PD and 15 normal controls where they performed submaximal exercise testing on a bicycle ergometer. Only one patient subject panicked and biochemical, physiological, and psychological data showed similar exercise tolerance in both patients and controls. A group from Norway tested the tolerance to intensive exercise and corresponding high levels of lactate in 35 patients with DSM-III-R diagnosis of PD (Martinsen, Raglin et al. 1998). The blood lactate values in this study were higher than the usually achieved values of 5 to $6 \mathrm{mmol} / \mathrm{L}$ during infusion where $67 \%$ of patients panic compared to $4 \%$ panicked in their study. Moreover, exercise may have a beneficial effect in PD when performed chronically. A German group from Göttingen randomized 46 patients with PD to either an exercise program, $112.5 \mathrm{mg} /$ day of clomipramine or placebo for 10 weeks (Broocks, Bandelow et al. 1998). They concluded that exercise was as effective as the pharmacological intervention and significantly superior to placebo. As a matter of fact, PD patients have a lower physical fitness when compared to normal subjects who are similarly sedentary (Meyer, Broocks et al. 1998).

Although $\mathrm{CO} 2$ challenges elicit small but consistent increases on panic scales in healthy subjects (Klaassen, Klumperbeek et al. 1998; Schruers, Pols et al. 2000), the present exploratory study was undertaken to test whether acute exercise may inhibit CO2 generated panic symptomatology in normal volunteers. 
The effects of acute exercise on $35 \% \mathrm{CO} 2$ induced panic in healthy volunteers

Methods

Students and university staff members were asked to participate in a study "about the interaction between $\mathrm{CO} 2$ breathing and exercise" in a casual setting. Trained athletes and individuals that exercised on a regular basis (weekly or more) were excluded. None of them was familiar with our laboratory's procedures. Inclusion criteria were a good present and past mental and physical condition, as assessed by a standardized general health questionnaire and a structured psychiatric interview (Sheehan, Lecrubier et al. 1998). Any use of psychotropic medication was excluded. The subjects were blind to the hypothesis under examination and randomized to either an exertion or a no-exertion control condition according to a separate group design. Mean (and SD) age was $29.5( \pm 5.91)$ year and $28.7( \pm 8.26)$ year for the former and the later group respectively. None of the above age and sex distribution differences was significant. Written informed consent was obtained from all subjects prior to the experiment.

On each subject, a heparinized venous catheter was placed in the cubital vein and a 2-ml-blood sample was taken for baseline lactate concentration. Afterwards the participants performed either the experimental condition or the control condition according to the randomization.

The experimental condition consisted of a physical activity performed on a bicycle ergometer for a 3-min warm-up at $70 \mathrm{rpm}$ with a workload of 100 watts $(W)$ for women and $125 \mathrm{~W}$ for men. This is a modified version of the Astrand-Ryming bicycle ergometer protocol (Astrand and Ryhming 1954). At the end of the 3-minute period, $2 \mathrm{ml}$. of blood was drawn from the catheter. Then the workload was increased to $175 \mathrm{~W}$ for women and $200 \mathrm{~W}$ for men for 9 min, drawing blood every 3 minutes. As soon as subjects reached a goal of $>6 \mathrm{mmol} / \mathrm{l}$ of lactate blood concentration exercise was terminated; if not the workload was increased $25 \mathrm{~W}$ for another 3 minutes and a blood sample was taken, and so on until the goal was reached or the subject was exhausted.

The control condition consisted of a physical activity on a bicycle ergometer with a continuous workload calculated at $1 \mathrm{~W} / \mathrm{kilo}$ of subject-20 W at $70 \mathrm{rpm}$. This is considered to be less then the workload required for normal walking on a leveled surface. Blood samples were taken every 3 minutes for a total of 12 minutes and the activity was terminated.

After either condition, the subjects were asked to relax in an armchair for 3 minutes. To assess the reactivity to $\mathrm{CO} 2$, they were then asked to fill-in the DSM-IV Panic Symptom List (PSL) and Visual Analogue Anxiety Scale (VAAS). A laboratory induced panic attack was not defined for this study. The last blood sample was taken to determine pre-CO2 challenge lactate level. Immediately afterwards an inhalation of a vital capacity using a mixture of $35 \%$ 
Chapter 2

$\mathrm{CO} 2 / 65 \% \mathrm{O} 2$ through a mask was give. Finally, the subjects were asked to fill-in the PSL and VAAS.

\section{Results}

All twenty subjects completed the experiment. All subjects completed the goal of reaching $>6 \mathrm{mmol} / \mathrm{l}$ of blood-lactate concentration. The average level at the moment of the $\mathrm{CO} 2$ challenge was $5.4( \pm 2.4) \mathrm{mmol} / \mathrm{l}$. and $1.1( \pm 0.2)$ in the exertion and control condition respectively, which were significantly different (two sample t-test, $p<0.01$, two tailed).

Exercise caused more physical symptoms as measured with the PSL than when compared with the control condition. Pre-CO2 PSL scores on exertion versus control was 5.8 $( \pm 4.15)$ and $1.8( \pm 2.09)$ respectively (two sample, $t$-test, $p<0.02$, two tailed). VAAS pre-CO2 scores on each condition were not significantly different.

To analyze the effects of exercise and the control condition with the $\mathrm{CO} 2$ challenge, the increases on PSL and VAAS scores were determined for each subject, by the difference in the 'post' minus 'pre'-CO2 inhalation. These scores showed a lower PSL for the exercise group when compared to the controls (See Table 1). Scores were $2.00( \pm 3.91)$ for the exertion group and $8.50( \pm 6.20)$ for the control group (two sample t-test, $p<0.02$, two tailed). For the VAAS (See Table 2), exertions versus control scores were $7.40( \pm 13.99)$ and $13.3( \pm 14.61)$, which were not significantly different (two sample t-test, $p=0.1$, two tailed). There was no correlation between lactate levels and PSL or VAAS scores.

Table 1. CO2 challenge results on the PSL scale

\begin{tabular}{llllllll}
\hline & Pre-CO2 & Post-CO2 & Delta & & Pre-CO2 & Post-CO2 & Deita \\
\hline & 6 & 13 & 7 & & 2 & 20 & 18 \\
& 4 & 10 & 6 & 3 & 8 & 5 \\
& 4 & 5 & 1 & & 0 & 11 & 11 \\
Exercise & 7 & 9 & 6 & 2 & 2 & 6 & 4 \\
$(n=10)$ & 3 & 9 & 2 & Controls & 1 & 14 & 13 \\
& 4 & 8 & 6 & $(n=10)$ & 1 & 16 & 15 \\
& 3 & 3 & 4 & & 7 & 9 & 2 \\
& 6 & 2 & 0 & -4 & 2 & 2 & 0 \\
& 17 & 13 & -4 & & 0 & 4 & 4 \\
\hline Mean & 5.8 & 7.8 & 2 & Mean & 1.8 & 10.3 & 8.5 \\
\hline SD & 4.15 & 3.79 & 3.91 & SD & 2.09 & 5.59 & 6.20 \\
\hline
\end{tabular}


The effects of acute exercise on $35 \% \mathrm{CO} 2$ induced panic in healthy volunteers

Table 2. $\mathrm{CO} 2$ challenge results on the VAAS scale

\begin{tabular}{|c|c|c|c|c|c|c|c|}
\hline & Pre-CO2 & Post-CO2 & Delta & & Pre-CO2 & Post-CO2 & Delta \\
\hline & 3 & 3 & 0 & & 15 & 62 & 47 \\
\hline & 5 & 25 & 20 & & 7 & 13 & 6 \\
\hline & 4 & 3 & -1 & & 14 & 20 & 6 \\
\hline & 16 & 44 & 28 & & 1 & 10 & 9 \\
\hline Exercise & 6 & 0 & -6 & Controls & 0 & 11 & 11 \\
\hline \multirow[t]{5}{*}{$(n=10)$} & 0 & 0 & 0 & $(n=10)$ & 23 & 35 & 12 \\
\hline & 0 & 0 & 0 & & 40 & 40 & 0 \\
\hline & 0 & 0 & 0 & & 3 & 4 & 1 \\
\hline & 0 & 0 & 0 & & 0 & 35 & 31 \\
\hline & 5 & 38 & 33 & & 3 & 13 & 10 \\
\hline Mean & 3.90 & 11.30 & 7.40 & Mean & 11.00 & 24.30 & 13.30 \\
\hline$S D$ & 4.89 & 17.47 & 13.99 & SD & 12.58 & 18.15 & 14.61 \\
\hline
\end{tabular}

Discussion

Exercise seemed to reduce the effects of a $\mathrm{CO} 2$ challenge. Compared to the control condition, the $\mathrm{CO} 2$ elicited increase in panic symptomatology, as expressed on the PSL scale was significantly reduced in the exertion group. As it could be expected from earlier studies (Klaassen, Klumperbeek et al. 1998; Schruers, Pols et al. 2000), this sample of healthy subjects reacted primarily with neurovegetative rather than psychological symptoms, so VAAS scores after $\mathrm{CO}_{2}$ showed no more than a trend towards reduction in the exercise group. Because a $\mathrm{CO} 2$ challenge hardly affects the anxiety level of normal individuals, a replication of the present results in a population of patients with Panic Disorder is warranted. Such a study should ideally demonstrate that, beside the somatic panic symptoms, exercise reduces CO2 induced anxiety as well.

Considering the present data, a number of caveats should be taken into account. We report a lower $\mathrm{CO} 2$ induced increase in PSL scores in the exertion group compared to controls. However, the exercise reduces $\mathrm{CO} 2$ caused PSL increase.

Subjects in the control condition had lactate levels comparable to those produced by lactate infusion (Liebowitz, Gorman et al. 1985), but an inhibitory rather than accumulative effect was seen with $\mathrm{CO} 2$. From the present data, a direct interaction between blood lactate and $\mathrm{CO} 2$ administration cannot be proven however. The observed effect could arise from mechanisms not directly linked to the pathophysiology of panic. For instance, subjects may be less aware of changes in interceptive sensations upon exercise since many sensation induced by physical effort do mimic some symptoms of panic. 
Chapter 2

Finally, it must be acknowledged that in the present small sample, no correlation could be evidenced between the level of blood lactate and the reduction in $\mathrm{CO} 2$ induced symptomatology.

The above reservations aside, the present pilot study may nevertheless reflect a genuine interplay between physical exertion and some mechanisms of experimental panic.

Klein (1993) developed a theory as to why $\mathrm{CO} 2$ and lactate produce panic, and explains that a misinterpretation of a CNS suffocation monitor fires in the presence of these substances. In terms of the fact that exercise is not panicogenic, Klein speculates that exertion provides countervailing information to this monitor, so suffocation cues such, as an elevation in lactate, will not produce panic. Accordingly, this countervailing signal may inactivate the suffocation monitor to such an extent that a 35\% CO2 challenge fails to produce its usual effect. From a different point of view, Deakin and Graeff (1991) postulate a balance between neuronal activity related to generalized anxiety and structures responsible of panic attacks. The increased arousal and anxiety during exercise (Cameron and Hudson 1986) may result in an elevated threshold for panic.

Future research with PD patients, CO2 challenge and acute exercise could provide insight into the pathophysiology of panic attacks. Studies have shown the benefit of exercise on PD and it would be worthwhile to elucidate the mechanisms linking muscular activity, panic and anxiety.

There is a great deal of research on the benefits of exercise on mental health, particularly mild to moderate mood and anxiety disorders (de Coverley Veale 1987; Paluska and Schwenk 2000). With these disorders being an important world prevalent cause of recurrent and lifelong physical and psychosocial impairment, an inexpensive and available-toall treatment such as exercise, might be an underused wonder. 
The effects of acute exercise on $35 \% \mathrm{CO} 2$ induced panic in healthy volunteers

\section{References}

Astrand, P. O. and I. Ryhming (1954). "A nomogram for calculation of aerobic capacity (physical fitness) from pulse rate during sub-maximal work." $J$ Appl Physiol 7(2): 218-21.

Broocks, A., B. Bandelow, et al. (1998). "Comparison of aerobic exercise, clomipramine, and placebo in the treatment of panic disorder." Am J Psychiatry 155(5): 603-9.

Cameron, O. G. and C. J. Hudson (1986). "Influence of exercise on anxiety level in patients with anxiety disorders." Psychosomatics 27(10): 720-3.

de Coverley Veale, D. M. (1987). "Exercise and mental health." Acta Psychiatr Scand 76(2): 113-20.

Deakin, J. F. W. and F. G. Graeff (1991). "5-HT and mechanisms of defence." J Psychopharmacol 5(4): 305-315.

Griez, E., C. de Loof, et al. (1990). "Specific sensitivity of patients with panic attacks to carbon dioxide inhalation." Psychiatry Res 31(2): 193-9.

Klaassen, T., J. Klumperbeek, et al. (1998). "Effects of tryptophan depletion on anxiety and on panic provoked by carbon dioxide challenge." Psychiatry Res 77(3): 167-74.

Klein, D. F. (1993). "False suffocation alarms, spontaneous panics, and related conditions. An integrative hypothesis." Arch Gen Psychiatry 50(4): 306-17.

Liebowitz, M. R., A. J. Fyer, et al. (1984). "Lactate provocation of panic attacks. I. Clinical and behaviora findings." Arch Gen Psychiatry 41 (8): 764-70.

Liebowitz, M. R., J. M. Gorman, et al. (1985). "Lactate provocation of panic attacks. II. Biochemical and physiological findings." Arch Gen Psychiatry 42(7): 709-19.

Martinsen, E.W., J. S. Raglin, et al. (1998), "Tolerance to intensive exercise and high levels of lactate in panic disorder." $J$ Anxiety Disord 12(4): 333-42.

Meyer, T., A. Broocks, et al. (1998). "Endurance training in panic patients: spiroergometric and clinical effects." Int J Sports Med 19(7): 496-502.

Paluska, S. A. and T. L. Schwenk (2000). "Physical activity and mental health: current concepts." Sports Med 29(3): 167-80.

Schruers, $K_{1}, H$. Pols, et al. (2000). " 10 microg CCK-4 premedication and $35 \%$ CO2 challenge in healthy volunteers." Prog Neuropsychopharmacol Biol Psychiatry 24(3): 409-18.

Sheehan, D. V., Y. Lecrubier, et al. (1998). "The Mini-International Neuropsychiatric Interview (M.I.N.I.): the development and validation of a structured diagnostic psychiatric interview for DSM-IV and ICD10." J Clin Psychiatry 59 Suppl 20: 22-33;quiz 34-57.

Stein, J. M., L. A. Papp, et al. (1992), "Exercise tolerance in panic disorder patients." Biol Psychiatry 32(3): $281-7$.

Verburg, K., H. Pols, et al. (1998). "Reliability of the $35 \%$ carbon dioxide panic provocation challenge." Psychiatry Res 78(3): 207-14. 
Chapter 3:

\title{
Acute exercise reduces the effects of experimental panic provocation with $35 \% \mathrm{CO} 2$ in patients with panic disorder
}

\author{
Esquivel $G^{1,2}$, Díaz-Galvis $J^{2}$, Schruers $K^{1}$, Berlanga $C^{2}$, Lara-Muñoz $C^{2}$ \\ and Griez $\mathrm{E}^{1}$
}

${ }^{1}$ Academic Anxiety Center, Department of Psychiatry and Neuropsychology, Maastricht University, Maastricht

2División de Investigaciones Clínicas, Instituto Nacional de Psiquiatría Ramón de la Fuente Muñiz, Mexico City

Published as: Esquivel, G., J. Diaz-Galvis, K. Schruers, C. Berlanga, C. Lara-Munoz and E. Griez (2008). "Acute exercise reduces the effects of a $35 \% \mathrm{CO}(2)$ challenge in patients with panic disorder." J Affect Disord 107(1-3): 217-20 
Acute exercise reduces the effects of $35 \% \mathrm{CO} 2$ in patients with panic disorder

\section{Abstract}

Background: Chronic exercise has been shown to have therapeutic effects in panic disorder (PD). The mechanism of these effects is unknown. Acute exercise reduces the effect of a panic challenge in healthy volunteers. Such an effect has not yet been demonstrated in PD patients. The present study aimed at exploring the antipanic effects of acute exercise on a $35 \%$ CO2 panic provocation in treatment-naïve PD patients to further elucidate the mechanisms of the beneficial effects of exercise on panic.

Methods: Eighteen PD patients performed either moderate/hard exercise or very-light exercise before a 35\% CO2 challenge in a randomized, between-group design. The reactivity to $\mathrm{CO} 2$ was assessed with the Visual Analogue Anxiety Scale and the DSM-IV Panic Symptom List. Results: Panic reactions to $\mathrm{CO} 2$ were smaller in patients that performed moderate/hard exercise in contrast to those that performed very-light exercise. Increments in both measurements and panic rates were consistently reduced by intense exercise.

Limitations: Since this study focuses on the acute effects of exercise on $\mathrm{CO} 2$ sensitivity in patients with $P D$, the results of repetitive exercise sessions on the rate of spontaneous panic attacks and overall symptoms is warranted.

Conclusions: Exercise reduced the panicogenic effects of a $\mathrm{CO} 2$ challenge. in addition to its therapeutic potential, exercise may also be useful as a laboratory maneuver with heuristic value in experimental research into the mechanisms of antipanic treatment.

Keywords: panic disorder, exercise, carbon dioxide. 
Chapter 3

Introduction

Exercise has long been regarded as a healthy behavior with both preventive and therapeutic properties for a variety of physical and mental pathological conditions (U.S. Dept. of Health and Human Services 1996). With regard to mental disorders, much focus has been placed on the relationship between exercise and affective disorders (De Moor, Beem et al. 2006; Harris, Cronkite et al. 2006). In line with the beneficial effects of regular physical activity on mental wellbeing, a 10-week exercise treatment program for panic disorder has shown to be as effective as a standard antipanic pharmacological intervention and superior to placebo (Broocks, Bandelow et al. 1998). It is however unclear if these beneficial effects were due to changes in aerobic metabolism or to the behavioral exposure effects of running therapy.

Intense acute physical activity induces changes in the anxious state of healthy and anxious individuals (de Coverley Veale 1987). In the case of panic disorder patients, anxious symptoms increase proportionally to the intensity of exercise when compared to healthy subjects (Stein, Papp et al. 1992). Yet unexpectedly, patients can well tolerate sub-maximal exercise causing lactate elevations that are comparable to those of a panicogenic lactate infusion (Stein, Papp et al. 1992). A pilot study with healthy volunteers, aimed at gaining insight in this apparently contradictory phenomenon, showed that acute exercise reduced the effect of a challenge with $35 \% \mathrm{CO} 2$, despite lactate elevations similar to those used in an infusion capable of provoking panic attacks (Esquivel, Schruers et al. 2002). Moreover, in other studies with normal subjects, acute exercise has shown to minimize the anxious symptoms provoked by both caffeine (Youngstedt, O'Connor et al. 1998) and cholecystokinin (CCK) (Strohle, Feller et al. 2005). A similar effect has not yet been documented in subjects with panic disorder. Accordingly, this study was designed to explore the antipanic effects of exercise on a $35 \%$ $\mathrm{CO} 2$ panic provocation in treatment-naive panic disorder patients.

\section{Methods}

Eighteen panic disorder patients from the outpatient clinic of the Instituto Nacional de Psiquiatría Ramón de la Fuente in Mexico City (eleven women and seven men; mean age 29.6 years, $S D=8.2$ ) were invited to voluntarily participate in the study. Patients with panic disorder (with or without agoraphobia in accordance to the DSM-IV) as main diagnosis made by at least two clinicians and a structured psychiatric interview (Sheehan, Lecrubier et al. 1998) were included for the study. All subjects were free of any serious or severe medical condition that could limit participation in the trial. Patients were excluded if they had previous use of any psychotropic medication, a severe mood disorder, a psychotic or substance-related disorder, or if they had more than three axis I diagnoses. Trained athletes and individuals that exercised 
on a regular basis (weekly or more) were also excluded. After complete description of the study to the subjects, written informed consent was obtained. The protocol was approved by the local medical ethics committee.

A blood sample was extracted from a finger puncture in each subject to determine basal lactate levels with a portable device (Accutrend portable lactate analyzer, Roche Diagnostics). Afterwards the participants performed either a moderate/hard exercise (exercise group) or a very-light exercise (control group) condition according to a randomized, between-group design. In the experimental condition, by using a modified version of the Astrand-Ryming bicycle ergometer protocol (Astrand and Ryhming 1954), patients performed a physical activity on a bicycle ergometer for a 3-min warm-up at $70 \mathrm{rpm}$ with a workload of 100 watts $(W)$ for women and $150 \mathrm{~W}$ for men. If unable to reach between 80 to $90 \%$ of their maximal heart rate at the end of the 3-minute period, 25 to $100 \mathrm{~W}$ were added to their previous workload. This process was repeated every minute until the target HR was reached and exercise was sustained until a total of 15 minutes elapsed or the patient was exhausted. The control condition consisted of a physical activity in the same fashion, but with minimal workload calculated at $1 \mathrm{~W}$ per $\mathrm{Kg}$ (weight)-20 at $70 \mathrm{rpm}$. Blood lactate was determined again before the termination of either condition. At the end of the test procedure subjects were asked to relax in an armchair to determine blood pressure and filled-in the DSM-IV Panic Symptom List (PSL) and Visual Analogue Anxiety Scale (VAAS). A third blood sample was taken to determine pre- $\mathrm{CO} 2$ challenge lactate levels. Immediately afterwards an inhalation of a vital capacity using a mixture of $35 \% \mathrm{CO} 2 / 65 \%$ O2 through a mask was given. Finally, the subjects were asked to fill-in the PSL and VAAS once more.

The reactivity to $\mathrm{CO} 2$ was assessed with the increments of subjective symptoms registered on the PSL and VAAS by subtracting the pre-CO2 scores to the post-CO2 scores. An experimental panic attack was defined by an increase of $25 \mathrm{~mm}$ or more on the VAAS and a rise of at least 4 symptoms on the PSL (Klaassen, Klumperbeek et al. 1998). Student's t-test was used to compare the means of parametric data. The means derived from the PSL and VAAS scores were analyzed with the Mann-Whitney U-test. Fisher's Exact test was used to compare the rate of panic attacks across the two groups. The level of significance was set at alpha $=0.5$ (two-tailed).

Results

Mean age of both experimental $(\mathrm{N}=10)$ and control $(\mathrm{N}=8)$ groups were not significantly different (30.1 years, $S D=8.9$; and 29.1 years, $S D=8.0 ; t=-0.241$, $d f=16, p=0.81$; respectively). Sex and comorbid axls I diagnosis distribution were similar among groups. All subjects with 2 
diagnoses $(N=11)$ had a mild to moderate major depressive disorder and those with 3 diagnoses additionally had social anxiety $(\mathrm{N}=1)$ and generalized anxiety disorder $(\mathrm{N}=1)$.

Figure 1. Panic responses measured with the Visual Analogue Anxiety Scale (VAAS) and the DSM-IV Panic Symptom List (PSL-IV), to a single inhalation with $35 \% \mathrm{CO} / 65 \%$ O2 in patients with panic dis-order after exercise or a control condition

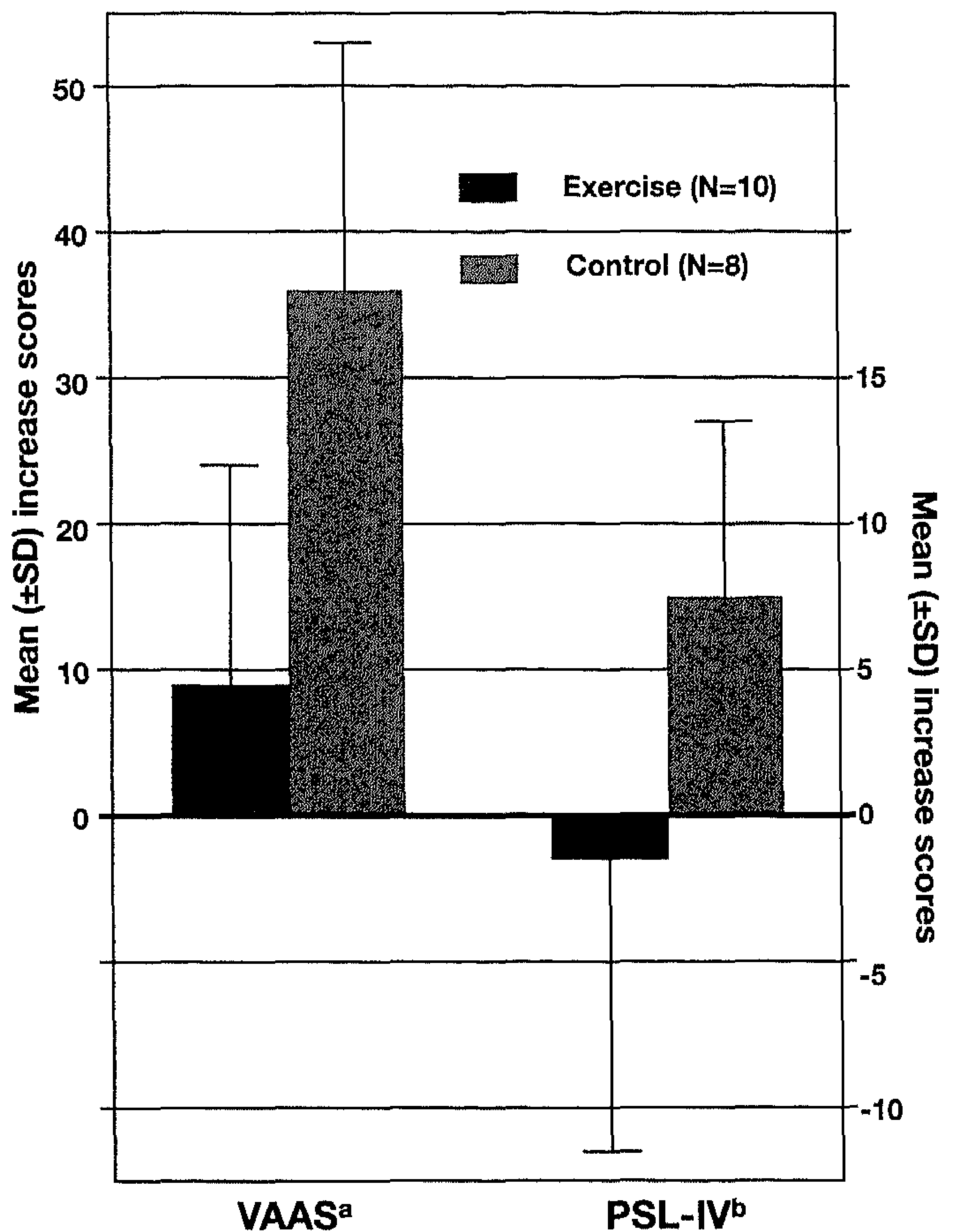

$a, b$ Significant difference be-tween experimental conditions as measured by the VAAS (Mann-Whitney $U=8.5, Z=-2.802, p<0.01$ ) and PSL (MannWhitney $U=9.0, Z=2.764, p<0.01$ ) 
Acute exercise reduces the effects of $35 \% \mathrm{CO} 2$ in patients with panic disorder

At the beginning of the session, blood lactate levels $(t=-1.688, d f=16, p=0.11), P S L$ scores (Mann-Whitney $U=30.0, Z=-0.894, p=0.37$ ) and VAAS scores (Mann-Whitney $U=34.5$, $Z=-0.492, p=0.62)$ were similar in both conditions. As expected, moderate/hard exercise elicited a significantly greater lactate elevation when compared with the control condition (9.56 $\mathrm{mmol} / \mathrm{L}, \mathrm{SD}=5.1 ; 3.08 \mathrm{mmol} / \mathrm{L}, \mathrm{SD}=0.98$; respectively $[t=3.917, \mathrm{df}=16, p<0.05]$ ). Intense physical activity elicited some of the neurovegetative symptoms listed in the PSL as the experimental group had a significantly larger score than the control group (mean=13.9, $S D=6.9$; and mean=4.8, $S D=4.1$; respectively; Mann-Whitney $U=10.0, Z=-2.672, p=0.008$ ). On the other hand, subjective anxiety as measured with the VAAS, was rather similar across groups (mean $=21.1, \mathrm{SD}=20.7$; and mean=16.3, $\mathrm{SD}=22.9$; exercise and control group respectively; Mann-Whitney $U=30.5, Z=-0.849, p=0.39$ ).

A single inhalation with $35 \% \quad \mathrm{CO} 2 / 65 \%$ O2 provoked a smaller panic reaction increase in patients who performed moderate/hard exercise when compared to the response of the control group (Figure 1). Increments in both the PSL (mean=-1.5, SD=10.4 versus mean=7.7, $S D=6.2$ ) and with the VAAS (mean=9.0, $S D=15.4$ vs. mean=36.2, $S D=17.9$ ) were consistently and significantly reduced in the exercise group. The panic rate was also lower in the exercise group (One of 10 versus five of eight, $p<0.05$, Fisher's exact test).

\section{Discussion}

Exercise reduced the panicogenic effects of a $35 \% \mathrm{CO} 2$ challenge in patients with PD. Compared to the control condition, the $\mathrm{CO} 2$ elicited increase in panic symptomatology expressed on the PSL and the VAAS was significantly reduced in the exercise group. Moreover, panic attacks were more frequent after very-light exercise than after moderate/hard exercise. As previously mentioned, acute exercise can reduce the effect of psychobiological anxiogenic maneuvers in healthy subjects such as the administration of caffeine (Youngstedt, O'Connor et al. 1998), CCK-4 (Strohle, Feller et al. 2005) and CO2 (Esquivel, Schruers et al. 2002). To our knowledge, this is the first report on the acute antipanic effect of exercise in patients with panic disorder. Patientswho preformed exercise reached lactate levels comparable to those produced by lactate infusion (Liebowitz, Fyer et al. 1984), but an inhibitory rather than accumulative effect was seen with $\mathrm{CO} 2$.

Several plausible mediators have been set forward to explain the antipanic effects of exercise (Strohle, Feller et al. 2005). Animal research has revealed a link between physical activity and changes in serotonergic function in raphe structures (Greenwood, Foley et al. 2005) that might contribute to the anxiolytic property of exercise. In accordance with studies suggesting a role for endorphins in panic (Sinha, Goetz et al. 2007), it is attractive to speculate that the effect of exercise on experimental panicogenic maneuvers may be mediated by these 


\section{Chapter 3}

peptides. A study with subjects under pre-medication with opiate antagonists or a tryptophan depletion may shed information on the role of both opiates and 5-HT in the antipanic effect of exercise. The administration of atrial natruiretic peptide (ANP), another neuropeptide, has shown to reduce the panicogenic effects of CCK (Strohle, Kellner et al. 2001). In this regard, a study with acute exercise has revealed that increases in ANP levels correlate with the exertion-induced reductions in the response to CCK in healthy volunteers (Strohle, Feller et al. 2006).

Fear is an ingrained function of the nervous system that coordinates behavioral and physiological actions to make survival a likelier outcome in the event of extreme danger. It is reasonable to think, from an adaptive perspective, that running (and therefore exercise) would not precipitate or exacerbate panic. Since this study focuses on the acute effects of exercise on $\mathrm{CO} 2$ sensitivity in patients with $\mathrm{PD}$, the results of repetitive exercise sessions on the rate of spontaneous panic attacks and overall symptoms is warranted. However, the results from this study suggest that acute exercise may not only represent an alternative form of therapy for patients with panic disorder, but may also be useful as a laboratory maneuver with heuristic value in experimental research into the mechanisms of antipanic treatment. 
Acute exercise reduces the effects of $35 \% \mathrm{CO} 2$ in patients with panic disorder

\section{References}

Astrand, P. O. and I. Ryhming (1954). "A nomogram for calculation of aerobic capacity (physical fitness) from pulse rate during sub-maximal work." $J$ Appl Physiol 7(2): 218-21.

Broocks, A., B. Bandelow, et al. (1998). "Comparison of aerobic exercise, clomipramine, and placebo in the treatment of panic disorder." Am J Psychiatry 155(5): 603-9.

de Coverley Veale, D. M. (1987). "Exercise and mental health." Acta Psychiatr Scand 76(2): 113-20.

De Moor, M. H. A. L. Beem, et al. (2006). "Regular exercise, anxiety, depression and personality: a population-based study." Prev Med 42(4): 273-9.

Esquivel, G., K. Schruers, et al. (2002). "The effects of acute exercise and high lactate levels on $35 \%$ CO2 challenge in healthy volunteers." Acta Psychiatr Scand 106(5): 394-7.

Greenwood, B. N., T. E. Foley, et al. (2005). "Wheel running alters serotonin (5-HT) transporter, 5-HT1A, $5-H T 1 B$, and alpha $1 \mathrm{~b}$-adrenergic receptor mRNA in the rat raphe nuclei." Biol Psychiatry $57(5)$ : $559-68$.

Harris, A. H., R. Cronkite, et al. (2006). "Physical activity, exercise coping, and depression in a 10-year cohort study of depressed patients." J Affect Disord 93(1-3): 79-85.

Klaassen, T., J. Klumperbeek, et al. (1998). "Effects of tryptophan depletion on anxiety and on panic provoked by carbon dioxide challenge." Psychiatry Res 77(3): 167-74.

Liebowitz, M. R., A. J. Fyer, et al. (1984). "Lactate provocation of panic attacks. I. Clinical and behavioral findings." Arch Gen Psychiatry 41 (8): 764-70.

Sheehan, D. V., Y. Lecrubier, et al. (1998). "The Mini-International Neuropsychiatric Interview (M.I.N.I.): the development and validation of a structured diagnostic psychiatric interview for DSM-IV and ICD10." J Clin Psychiatry 59 Suppl 20: 22-33;quiz 34-57.

Sinha, S. S., R. R. Goetz, et al. (2007). "Physiological and behavioral effects of naloxone and lactate in normal volunteers with relevance to the pathophysiology of panic disorder." Psychiatry Res 149(1-3): 309-14.

Stein, J. M., L. A. Papp, et al. (1992). "Exercise tolerance in panic disorder patients." Biol Psychiatry 32(3): $281-7$.

Strohle, A., C. Feller, et al. (2005). "The acute antipanic activity of aerobic exercise." Am J Psychiatry 162(12): 2376-8

Strohle, A., C. Feller, et al. (2006). "Anxiety modulation by the heart? Aerobic exercise and atrial natriuretic peptide." Psychoneuroendocrinology 31(9): 1127-30.

Strohle, A., M. Kellner, et al. (2001). "Anxiolytic activity of atrial natriuretic peptide in patients with panic disorder." Am J Psychiatry 158(9): 1514-6.

U.S. Dept. of Health and Human Services (1996). Physical activity and health: a report of the Surgeon General, Atlanta, GA., U.S. Dept. of Health and Human Services, Centers for Disease Control and Prevention.

Youngstedt, S. D., P. J. O'Connor, et al. (1998). "Acute exercise reduces caffeine-induced anxiogenesis." Med Sci Sports Exerc 30(5): 740-5. 
Chapter 4:

Effects of exercise on models of $\mathrm{CO} 2$ induced panic

Esquivel G, Schruers K, Dandachi A and Griez E

Academic Anxiety Center and School for Mental Health and Neurosciences,

Faculty of Medicine, Health and Life Sciences, Maastricht University,

Maastricht, The Netherlands 
Abstract

Background: Acute exercise has shown to reduce the effects of experimental panic provocation in healthy volunteers and patients with panic disorder. Recent evidence suggests that when larger amounts of $\mathrm{CO} 2$ are inhaled, a large proportion of healthy subjects can also have an affective response consistent with definitions of a panic attack. Our aim was to test whether exercise can show antipanic effects in healthy subjects when exposed to higher concentrations of $\mathrm{CO} 2$.

Methods: Thirty-one healthy subjects, on four separate occasions in a randomized Latin square design, performed either moderate/hard or very-light exercise immediately followed by either a single or a double $35 \% \mathrm{CO} 2 / 65 \% \mathrm{O} 2$ inhalation.

Results: Compared to very-light exercise, when subjects performed moderate/hard exercise they reported a larger reduction in panic symptoms than controls after a $\mathrm{CO} 2$ challenge on Panic Symptom List and the Visual Analogue Scale of Anxiety but no difference on the Visual Analogue Scale of Discomfort. The antipanic effect of exercise was not different across $\mathrm{CO} 2$ dosages.

Conclusions: After intense exercise, subjects had less panic when exposed to both a single and a double $35 \% \mathrm{CO} 2$ inhalation.

Key words: carbon dioxide, exertion, exercise, panic disorder, anxiety. 
Chapter 4

Introduction

The $35 \%$ carbon dioxide inhalation is a well established and validated method to experimentally induce panic attacks in patients with panic disorder Nerburg, Griez et al. 1995; Perna, Bertani et al. 1997; Verburg, Pols et al. 1998; Perna, Casolari et al. 2004). Recent evidence suggest that when larger amounts of $\mathrm{CO} 2$ are inhaled, healthy subjects have an affective response consistent with current nosologic definitions of a panic attack (Griez, Colasanti et al. 2007). Modeling experimental panic maneuvers in healthy subjects has important implications as a tool to evaluate the antipanic potential of novel compounds and strategies as well as their underlying mechanisms.

Acute exercise has shown to reduce the effects of experimental panic provocation in healthy volunteers with both $\mathrm{CO} 2$ inhalation and CCK administration (Esquivel, Schruers et al. 2002; Strohle, Feller et al. 2005; Smits, Meuret et al. 2008). When healthy participants are subjected to a single inhalation with $35 \% \mathrm{CO} 2$, exercise exerts a modest antipanic effect characterized by reductions of physical components of panic. In contrast, when the same procedures are performed in patients with panic disorder, who usually have a larger panic response to $\mathrm{CO} 2$ inhalations, acute exercise has a strong antipanic effect with reductions in both physical symptoms and subjective anxiety (Esquivel, Diaz-Galvis et al. 2008). In this study, we test whether acute exercise will display antipanic effects when healthy volunteers are subjected to larger doses of $\mathrm{CO} 2$.

\section{Methods}

\section{Subjects}

Thirty-one healthy volunteers ( 18 women and 13 men; mean age 23.90 years, $S D=5.74$ ) were recruited via public advertisement. All subjects where in good mental and physical health. Participants were excluded if they smoked more than 10 cigarettes a day or if they have previous use of any psychotropic medication. After complete description of the study, a written informed consent was obtained. The local medical ethics committee approved the conduction of this study.

\section{Experimental design}

The initial evaluation consisted of a MINI interview and a brief physical examination. Afterwards a baseline of reactivity to $\mathrm{CO} 2$ was obtained to familiarize subjects with the overall procedure. To perform a $\mathrm{CO} 2$ challenge, subjects were asked to take a single vital capacity inhalation through a mask using a mixture of $35 \% \mathrm{CO} 2$ / 65\% O2 and then breath-hold for four seconds. Care was taken that each inhalation represented at least $80 \%$ of the subject's vital capacity. 
Effects of exercise on models of $\mathrm{CO} 2$ induced panic

Subsequently, participants performed brief exercise sessions followed by a $\mathrm{CO} 2$ challenge on four occasions separated by 3 to 7 days. On each occasion, participants performed either moderate/hard exercise or a very-light exercise immediately before either a single or a double CO2 challenge inhalation according to a randomized, 4-period cross-over design (Latin square design). A blood sample was extracted from a finger puncture in each subject to determine basal lactate levels with a portable device (Accutrend portable lactate analyzer, Roche Diagnostics). During the sessions, by using a standard sub-maximal bicycle ergometer testing (American College of Sports Medicine., Whaley et al. 2006), subjects performed enough physical activity to reach $70 \%$ of their maximal heart rate. At this point a finger puncture blood sample was taken. Exercise was sustained and workload increased by $20 \mathrm{~W}$ every 3 minutes until blood lactate level reached more then $6 \mathrm{mmol} / /$ (determined by subsequent finger puncture blood samples) or the subject was exhausted.

\section{Assessment}

To measure panic symptoms, participants filled-in the DSM-IV Panic Symptoms List (PSL) and the Visual Analogue Scales for Anxiety and Discomfort (VAS-A and VAS-D) before the exercise session as well as before and immediately after the $\mathrm{CO} 2$ challenge. To analyze the reactivity to $\mathrm{CO} 2$, the increases on PSL, VAS-A and VAS-D scores were determined for each subject by subtracting the scores obtained immediately before the challenge to those after the challenge. An increase of $25 \mathrm{~mm}$ or more on the VAS-A and a rise of at least 4 symptoms on the PSL were used as criteria for a positive challenge response. This threshold is consistent with conservative criteria proposed by others to define responders in studies with $\mathrm{CO} 2$ (Battaglia and Perna 1995; Poma, Milleri et al. 2005). As primary outcome measure, the reactivity to $\mathrm{CO} 2$ was assessed with the increments of subjective symptoms registered on the PSL and VAS by subtracting the pre-CO2 scores to the post-CO2 scores. As a secondary measure, the symptoms elicited by exercise itself will be assessed with the increments on these same scales by subtracting the scores before exercise to the scores recorded after exercise.

\section{Statistical analyses}

A two-way multivariate analysis of variance (MANOVA) with repeated measures design was used to test the differences in primary and secondary outcome measures between conditions. Exercise intensity and $\mathrm{CO} 2$ dose were within-subjects factors each with two levels. Hypothesis tests for main and interaction effects of these factors were based on multivariate criteria. Univariate $\mathrm{F}$ tests were used to investigate simple effects when significant main or interactions effects were found. The McNemar paired chi-square test was used to compare the rate of panic attacks across the two conditions. The level of significance for all tests was set at alpha $\leq 0.05$ (two-tailed). 
Results

All 31 subjects completed the experiment. During the moderate/hard condition, all subjects completed the goal of reaching $>6 \mathrm{mmol} / /$ of blood-lactate concentration or exhaustion. As expected, the average blood lactate level immediately after exercise was higher during the high/moderate condition than in the very-light condition, $9.6(S D=2.0) \mathrm{mmol} / \mathrm{l}$. and 4.8 $(S D=1.8)$ respectively $(t=7.730, d f=19, p<0.001$, two tailed). The mean increments on outcome measures due to exercise alone and due to the $\mathrm{CO} 2$ challenge are reported in tables 1 and 2 , respectively.

Table 1. The mean increments on outcome measures due to exercise alone

\begin{tabular}{llllllll}
\hline Condition & & $\Delta$ PSL & \multicolumn{3}{c}{$\Delta$ VAS-A } & \multicolumn{3}{c}{$\Delta$ VAS-D } \\
\cline { 3 - 8 } & & Mean & SD & Mean & SD & Mean & SD \\
\hline (Single inhalation) & Very-light exercise & 1.12 & 1.14 & 0.74 & 2.01 & 0.93 & 2.17 \\
& Moderate/Hard exercise & 5.48 & 2.70 & 0.70 & 2.14 & 6.80 & 9.05 \\
\hline (Double inhalation) & Very-light exercise & 0.90 & 1.16 & 0.87 & 2.17 & -0.06 & 3.14 \\
& Moderate/Hard exercise & 5.67 & 2.56 & 1.64 & 4.34 & 5.48 & 7.20 \\
\hline
\end{tabular}

PSL: Panic Symptom List; VAS-A: Visual Analogue Scale for Anxiety; VAS-D: Visual Analogue Scale for Discomfort. Mean increment or delta scores due to exercise alone were calculated by subtracting the scores before exercise to the scores recorded after exercise

Table 2. The mean increments on outcome measures due to the $\mathrm{CO} 2$ challenge

\begin{tabular}{llllllll}
\hline Condition & \multicolumn{1}{c}{$\Delta$ PSL } & \multicolumn{3}{c}{$\Delta$ VAS-A } & \multicolumn{2}{c}{$\Delta$ VAS-D } \\
\cline { 3 - 8 } & & Mean & SD & Mean & SD & Mean & SD \\
\hline Single inhalation & Very-light exercise & 8.32 & 7.37 & 23.03 & 28.17 & 32.00 & 31.37 \\
& Moderate/Hard exercise & 4.96 & 7.06 & 18.48 & 28.41 & 31.77 & 36.52 \\
\hline \multirow{2}{*}{ Double inhalation } & Very-light exercise & 10.77 & 7.75 & 25.45 & 29.76 & 40.54 & 31.75 \\
& Moderate/Hard exercise & 6.06 & 6.46 & 19.54 & 22.63 & 35.87 & 27.76 \\
\hline
\end{tabular}

PSL: Panic Symptom List; VAS-A: Visual Analogue Scale for Anxiety; VAS-D: Visual Analogue Scale for Discomfort. Mean increment or delta scores due the $\mathrm{CO} 2$ challenge were calculated by subtracting the pre-CO2 scores to the post-CO2 scores

The effects of exercise on the $\mathrm{CO} 2$ challenge

MANOVA's of all outcome variables show significant main effects of exercise ( $F=7.770 ; d f=3$, $28 ; p=0.001)$. The main effects of $\mathrm{CO} 2$ and the interaction effects of exercise by $\mathrm{CO} 2$ were not significant. Univariate tests reveal that the effects of exercise on the challenge was significant for the PSL ( $F=22.55 ; d f=1,30 ; p<0.001)$, the VAS-A ( $F=5.49 ; d f=1,30 ; p=0.026)$, but not for VAS-D. These effects of exercise were due to overall lower panic measurement scores after 
Effects of exercise on models of $\mathrm{CO} 2$ induced panic

moderate/high exercise (Fig. 1). There were no significant differences in the rate of panic attacks between exercise conditions $(9$ versus 6 panic attacks, very-light versus hard/moderate exercise respectively, single inhalation; and 11 versus 10 , very-light versus hard/moderate exercise respectively, double inhalation).

Figure 1. Estimated marginal means in outcome measurement scales after single and double $35 \% \mathrm{CO} 2$ inhalations immediately after either very-light or moderate/hard exercise

VAS*A

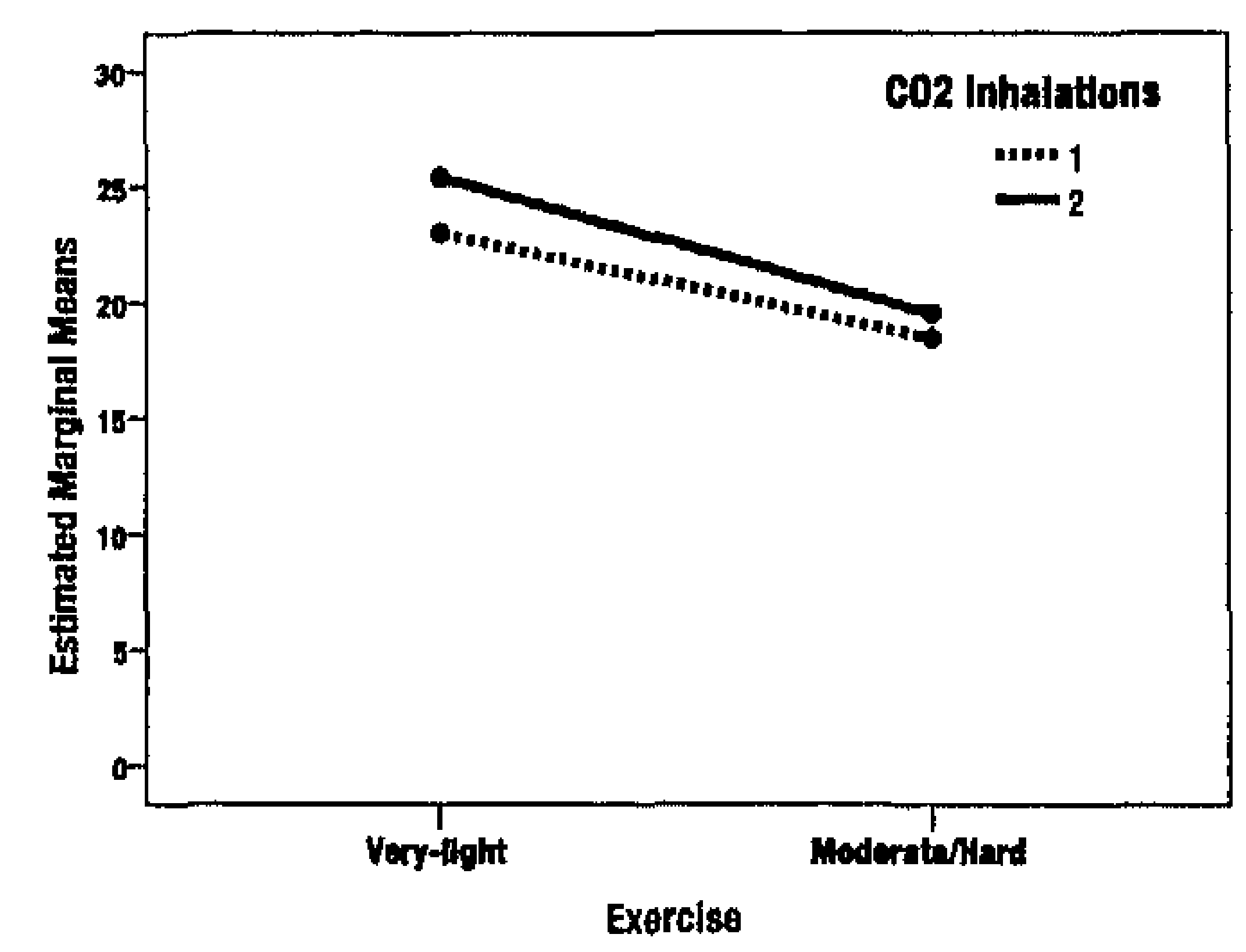

VAS-D
PSL

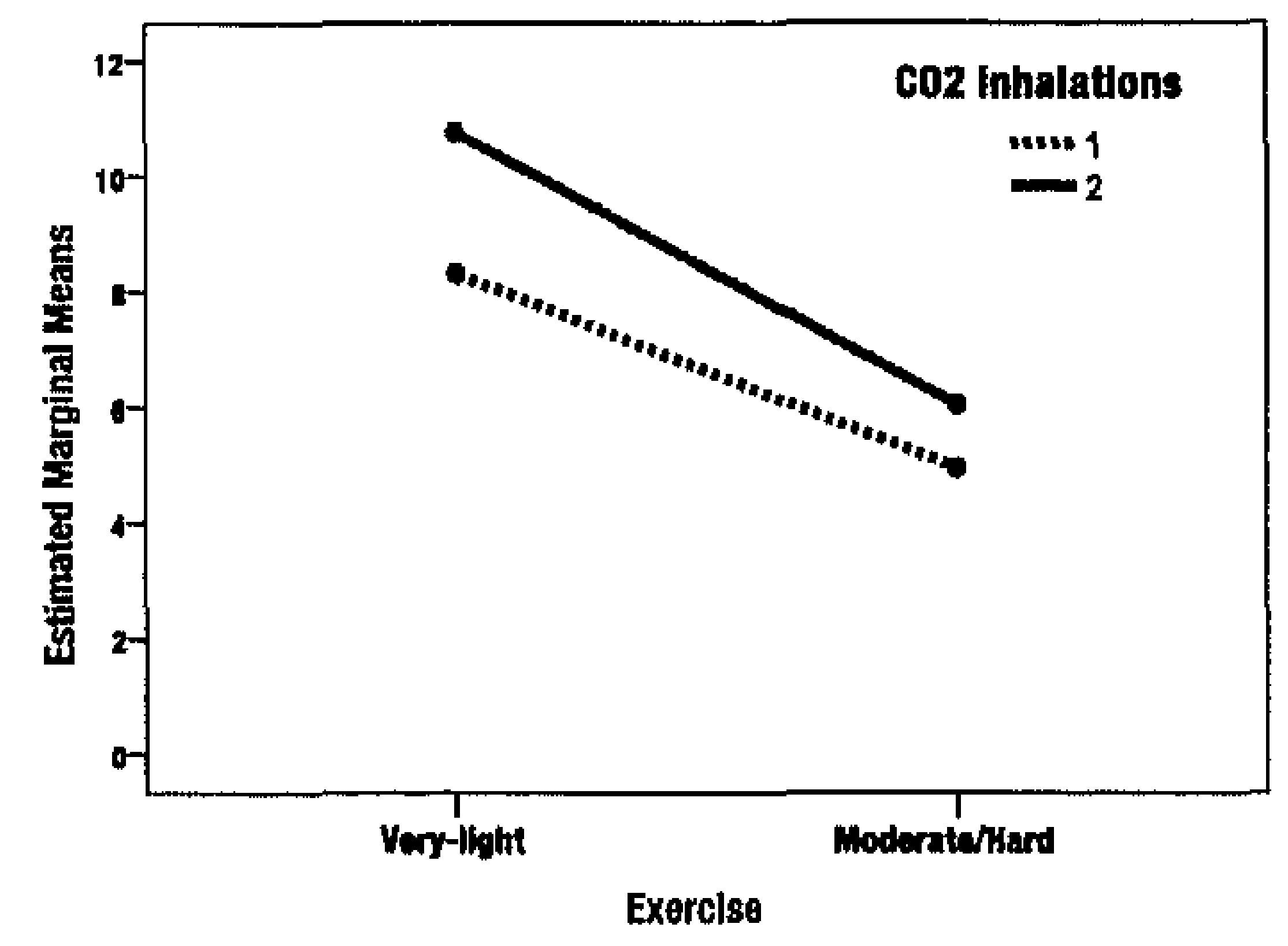

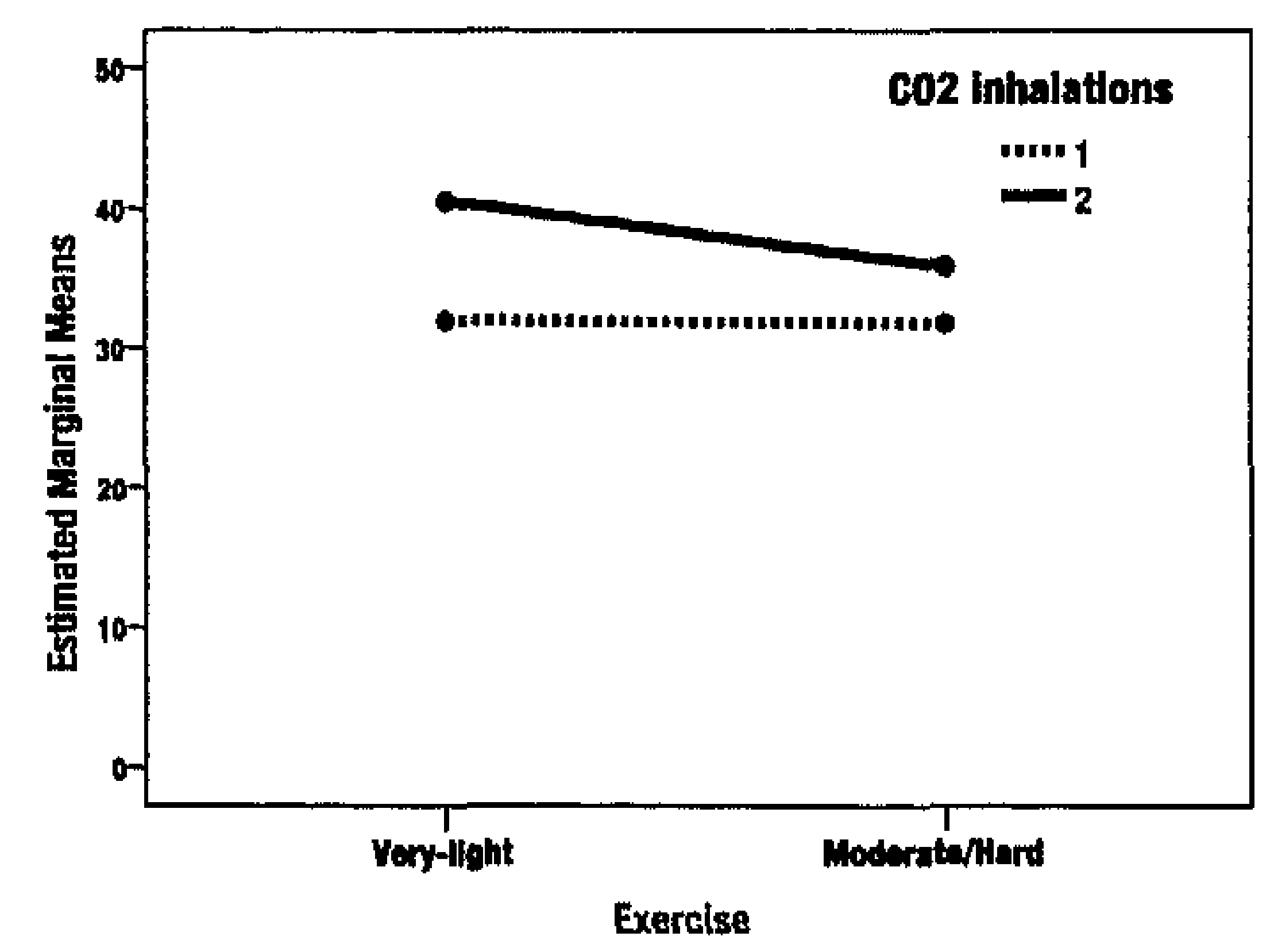

PSL: Panic Symptom List; VAS-A: Visual Analogue Scale for Anxiety; VAS-D: Visual Analogue Scale for Discomfort 
The effects of exercise alone

With regard to the effects of exercise alone on the outcome scales, MANOVA's also show significant main effects of exercise alone $(F=55.86 ; d f=3,28 ; p<0.001)$ and, as expected, no main effects of $\mathrm{CO} 2$ and the interaction effects of exercise alone by $\mathrm{CO} 2$. Univariate tests reveal that the effects of exercise was significant for the PSL $(F=167.22 ; d f=1,30 ; p<0.001)$, the VAS-D ( $F=24.60 ; d f=1,30 ; p<0.001)$, but not for VAS-A. These effects of exercise alone were due to overall higher PSL and VAS-D scores after moderate/high exercise (Tab. 2). Notably, exercise alone did not increase anxiety as rated in the VAS-A.

\section{Discussion}

In our sample of healthy subjects, a single episode of intense exercise reduces the panic response to a $\mathrm{CO} 2$ challenge. The protective effects of exercise were observed on the scores reported in the PSL and VAS-A. The lack of significant interaction effects of exercise level by $\mathrm{CO} 2$ dosage suggests that the protective effects of exercise can be modeled using both a single and double $35 \% \mathrm{CO} 2$ inhalation.

These results are, in part, consistent with our earlier findings (Esquivel, Schruers at al. 2002) and the findings produced independently by other authors (Smits, Meuret et al. 2008). We now see that, exercise has broader antipanic effects, in healthy subjects than what our former study had suggested. This can be explained by the increased power from the use of a larger sample size and a repeated-measures design. For instance, Smits and collaborators (2008) in a comparatively large sample size of healthy subjects $(\mathrm{N}=92)$, found that acute exercise reduced the effects of a single $35 \% \mathrm{CO} 2$ inhalation on both Acute Panic Inventory total scores and the scores of subjective units of distress (roughly analogous to the use of the PSL and VAS-A in the present study).

As in our former studies, exercise alone increased the scores in the many items of the PSL related to physical symptoms that are in common with panic symptoms. Since our outcome measure is defined as an increase in scale scores obtained by subtracting scores immediately before the $\mathrm{CO} 2$ challenge to those reported immediately afterwards, our results can be potentially influenced by the symptoms elicited by exercise. Nevertheless, besides the fact that high scores before a CO2 challenge usually do not minimize the effect of a $35 \% \mathrm{CO} 2$ inhalation and no ceiling effect was observed in our sample of healthy subjects. Moreover, moderate/hard exercise alone did not increase the scores reported on the VAS-A immediately before exercise, yet reduced the increase produced by the $\mathrm{CO} 2$ inhalations.

The lack of main effects of $\mathrm{CO} 2$ dose was unexpected but not entirely surprising. Although main effects of $\mathrm{CO} 2$ did not show a statistically significant effect according to multivariate criteria, uncorrected univariate $F$ tests showed a significant effects for scores on 
the PSL ( $F=4.912 ; d f=1,30 ; p<0.034)$; those scores being larger with a double inhalation. The dose-response effect of $\mathrm{CO} 2$-induced negative affectivity has been well established in our lab (Griez, Colasanti et al. 2007, Schruers, Esquivel et al. Submittted), where the effects of increasing the $\mathrm{CO} 2$ concentration are perhaps more robust than increasing the number of inhalations from 1 to 2 .

Relevant issues can be addressed with the results from this study. First, a double inhalation with $35 \% \mathrm{CO} 2$ is usefully to model the acute antipanic effects of exercise in healthy subjects. Encouragingly, perhaps other forms of antipanic interventions can be modeled in healthy subjects with a double-35\% CO2 inhalation. Second, although the well-sized study reported by Smits et al supports our earlier findings in healthy subjects, their control condition consisting of simple rest left the need to demonstrate these effects while controlling for nonspecific exercise effects with a low or very-light exercise condition.

Aerobic exercise has gained increasing interest as potential strategy to treat patients with mental ailments including panic disorder. Although much research is needed to establish exercise as an alternative evidenced-based treatment for panic disorder, the use of acute exercise in experimental settings may have heuristic value into antipanic mechanisms. For instance, mediation of the serotonergic and endorphinic systems in the acute anti-panic effects of exercise can be tested using well-established pharmacological manipulations. With the potential of aerobic exercise has as a low-cost, stigma-free and readily available alternative anti-panic strategy with additional direct health benefits for patients suffering from panic attacks, the need to better understand the complex relationship between physical activity and mental health is timely. 
Chapter 4

\section{References}

American College of Sports Medicine., M. H. Whaley, et al. (2006). ACSM's quidelines for exercise testing and prescription. Philadelphia, Lippincott Williams \& Wilkins.

Battaglia, M. and G. Perna (1995). "The $35 \% \mathrm{CO} 2$ challenge in panic disorder: optimization by receiver operating characteristic (ROC) analysis." J Psychiatr Res 29(2): 111-9.

Esquivel, G., J. Diaz-Galvis, et al. (2008). "Acute exercise reduces the effects of a $35 \% \mathrm{CO}(2)$ challenge in patients with panic disorder." J Affect Disord 107(1-3): 217-20.

Esquivel, G., K. Schruers, et al. (2002). "The effects of acute exercise and high lactate levels on $35 \% \mathrm{CO} 2$ challenge in healthy volunteers." Acta Psychiatr Scand 106(5): 394-7.

Griez, E. J., A. Colasanti, et al. (2007). "Carbon dioxide inhalation induces dose-dependent and agerelated negative affectivity." PLOS ONE 2(10): e987.

Perna, G., A. Bertani, et al. (1997). "Modffication of 35\% carbon dioxide hypersensitivity across one week of treatment with clomipramine and fluvoxamine: a double-blind, randomized, placebocontrolled study." J Clin Psychopharmacol 17(3): 173-8.

Perna, G., A. Casolari, et al. (2004). "Comparison of $35 \%$ carbon dioxide reactivity between panic disorder and eating disorder." Psychiatry Res 125(3): 277-83.

Poma, S. Z., S. Milleri, et al. (2005). "Characterization of a $7 \%$ carbon dioxide (CO2) inhalation paradigm to evoke anxiety symptoms in healthy subjects." J Psychopharmacol 19(5): 494-503.

Smits, J. A., A. E. Meuret, et al. (2008). "The effects of acute exercise on CO(2) challenge reactivity." $\underline{J}$ Psychiatr Res.

Strohle, A., C. Feller, et al. (2005). "The acute antipanic activity of aerobic exercise." Am J Psychiatry 162(12): 2376-8.

Verburg, K., E. Griez, et al. (1995). "Discrimination between panic disorder and generalized anxiety disorder by 35\% carbon dioxide challenge." Am J Psychiatry 152(7): 1081-3.

Verburg, K., H. Pols, et al. (1998). "Reliability of the $35 \%$ carbon dioxide panic provocation challenge." Esychiatry Res 78(3): 207-14. 
Chapter 5:

\title{
The effects of opioid receptor blockade on experimental panic provocation with $35 \% \mathrm{CO} 2$
}

\author{
Esquivel $\mathrm{G}^{1}$, Fernández-Torre $\mathrm{O}^{2}$, Schruers $\mathrm{K}^{1}$, Wijnhoven LLW' \\ and Griez $E^{1}$ \\ 'Maastricht University, School for Mental Health and Neurosciences and \\ Academic Anxiety Center, The Netherlands \\ ${ }^{2}$ University Hospital Marqués de Valdecilla, Department of Psychiatry, \\ Santander, Spain
}

Published as: Esquivel, G., O. Fernández-Torre, K. R. J. Schruers, L. L. W. Wijnhoven and E. J. L. Griez (2008). "The effect of opioid receptor blockade on experimental panic provocation with CO2." J Psychopharmacol Ahead of print: 1-4 
The effect of opioid receptor blockade on experimental panic provocation with $35 \% \mathrm{CO} 2$

\begin{abstract}
Background: Several reports have linked, among other aspects, the role of an opioid system in respiratory physiology with underlying mechanisms of panic attacks. The involvement of the opioid system in experimental panic is to be further probed. This study aimed to determine if opioid blockade would increase panic related symptomatology on provocation with $35 \%$ carbon dioxide inhaled by healthy volunteers.

Methods: Participants in a double-blind, randomized, crossover design orally received either naltrexone $50 \mathrm{mg}$ or placebo. Most subjects undertook a 35\% $\mathrm{CO} 2$ double inhalation one hour after pre-medication and a separate group did so after 5 hours. The reactivity to $\mathrm{CO}_{2}$ and the symptoms elicited by naltrexone alone were measured.

Results: Among other findings, naltrexone pre-medication alone elicited significant increments in panic related symptoms. Responses to $\mathrm{CO} 2$ were not significantly different between conditions in either group. Conclusions: These preliminary findings suggest that exposure to opioid blockade alone can potentially elicit symptoms the resemble panic, however, without modifying the response to experimental panic provocation with $35 \% \mathrm{CO} 2$.
\end{abstract}

Keywords: opioids, naltrexone, panic, 35\% CO2 inhalation, anxiety. 


\section{Chapter 5}

\section{Introduction}

The underlying mechanisms by which many different techniques can experimentally induce panic attacks in subjects with panic disorder remain elusive. Since several of these techniques are considered to be respiratory challenges (i.e. lactate, CO2, doxapram), the role of opioids may be linked to their effects on the perceived discomfort with breathing. For instance, opioid blockade can increase the sensations of dyspnea to acute respiratory stimulation (Akiyama, Nishimura et al. 1993) and opioids such as morphine have been extensively used to treat dyspnea (Jennings, Davies et al. 2002). Nonetheless, an earlier study in panic disorder patients failed to show any change in the susceptibility to a lactate infusion after pre-medication with naloxone (Liebowitz, Gorman et al. 1984). Sinha et al. (2007) have recently interpreted those findings as a possible opioid deficiency in patients with panic where opioid blockade would have a small effect in a previously incapacitated system. Accordingly, this research group designed a similar study however using healthy volunteers who were expected to have an intact opioid system. Results from this study, although preliminary, show that total measures of panic, breathlessness and ventilatory response significantly increased.

These and other findings have encouraged Preter and Klein (2008) to expand the suffocation false alarm theory and postulate that an endorphinergic dysfunction has a major role decreasing the alarm threshold. Consistent with this proposal, two case reports describe the onset of spontaneous panic attacks a few hours after the initiation of treatment with oral opioid antagonist in eating disorder patients with no prior history of panic attacks (Luby and Marrazzi 1987; Maremmani, Marini et al. 1998).

The purpose of this study was to explore the role of endogenous opioids in experimental panic provocation with carbon dioxide in healthy subjects. Correspondingly we tested the hypothesis that opioid blockade, by means of oral pre-medication with naltrexone, would increase the panic reactivity to a double inhalation of $35 \% \mathrm{CO} 2$ in healthy volunteers.

\section{Methods}

\section{Subjects}

Twenty-four healthy volunteers (eight women and eight men, mean age 24.7 years, SD=5.2; and a separate group of eight females, mean age 22.5 years, $S D=3.1$ ) were recruited via public advertisement. All participants were in good physical health and had no present or past history of mental disorders. Additional exclusion criteria included the presence of a history of pulmonary or cardiovascular disease, cerebral aneurism, epilepsy, pregnancy, excessive smoking (>10 cigarettes/day) and the use of any psychotropic or opioid medication. After 
The effect of opioid receptor blockade on experimental panic provocation with $35 \% \mathrm{CO} 2$

complete description of the study to the subjects, written informed consent was obtained. The study was approved by the local medical ethics committee.

\section{Procedures and design}

Screening. After an initial debriefing, subjects underwent a brief physical examination and a structured diagnostic interview (Sheehan, Lecrubier et al. 1998) performed by a psychiatrist. Additionally, a $\mathrm{CO} 2$ challenge was given to familiarize subjects with the overall procedure. To perform a $\mathrm{CO} 2$ challenge, subjects were asked to take two consecutive vital capacity inhalations through a mask using a mixture of $35 \% \mathrm{CO} 2 / 65 \% \mathrm{O} 2$ and hold the second inhalation for four seconds. Care was taken that each inhalation represented at least $80 \%$ of the subject's vital capacity.

Test phase. One week after screening, subjects orally received a capsule with either naltrexone $50 \mathrm{mg}$ or placebo in accordance to a double-blind, randomized, crossover design. On a subsequent visit (one week washout period) participants received the alternative capsule. Sixteen subjects underwent a $\mathrm{CO} 2$ challenge one hour after the drug or placebo administration. A separate group of eight females was subjected to a $\mathrm{CO} 2$ challenge 5 hours after the administration of placebo or naltrexone. This latter group was included to control for prolonged exposure to opioid blockade as two female subjects from the former group reacted strongly to the medication alone several hours after administration.

\section{Assessment instruments}

To measure panic symptoms, subjects filled-in the DSM-IV Panic Symptom List (PSL) and the Visual Analogue Scales for Anxiety and Discomfort NAS-A and VAS-D) before the capsule administration as well as before and immediately after the CO2 challenge. An increase of 25 $\mathrm{mm}$ or more on the VAS-A and a rise of at least 4 symptoms on the PSL were used as criteria for a positive challenge response. This threshold is consistent with conservative criteria proposed by others to define responders in studies with CO2 (Battaglia and Perna 1995; Poma, Milleri et al. 2005). Medication side-effects were recorded with a complaint list where subjects rated the severity of 31 possible adverse effects. As primary outcome, the reactivity to $\mathrm{CO} 2$ was assessed with the increments of subjective symptoms registered on the PSL and VAS by subtracting the pre-CO2 scores to the post-CO2 scores. As a secondary outcome, the symptoms elicited by naltrexone itself was assessed with the increments on these same scales by subtracting the scores before the drug administration to the scores recorded one hour after or five hours in the case of the group of 8 females. 
Statistical analyses

The differences in primary and secondary outcome measures between conditions were compared using a t test for paired-samples. The McNemar paired chi-square test was used to compare the rate of panic attacks across the two conditions. The relationship between the increases in panic severity scales after naltrexone alone and the total number of reported sideeffects was analyzed using Pearson's correlation coefficient. The level of significance for all tests was set at alpha $\leq 0.05$ (two-tailed). Additionally, the Bonferroni procedure was used to correct for the use of multiple measures (PSL, VAS-A and VAS-D).

\section{Results}

The scores produced on panic measures by both groups during the different time periods are presented in Table 1. After the $\mathrm{CO} 2$ challenge, increases on the number of panic symptoms (PSL) and reported anxiety or discomfort (VAS-A and VAS-D) were not significantly different between placebo and naltrexone in both the one-hour and five-hour groups (Table 1). Additionally, McNemar tests revealed no differences in rates of positive challenge responses between conditions (four of 16 vs. five of 16 and four of eight vs. five of eight, placebo vs. naltrexone respectively).

With regard to the effects of naltrexone alone on panic measures (Table 1), one-hour pre-medication only revealed significant increases in subjective discomfort $(t=-2.279, d f=15$, $P=0.038$ ). On the other hand, five hours of naltrexone elicited significant increments on the PSL and VAS-D ( $t=-3.347, d f=7, P=0.012 ; t=-2.534, d f=7, P=0.039 ;$ respectively) and marginally significant increments on the VAS-A $(t=-2.098, d f=7, P=0.075)$. When scrutinized under conservative criteria, only increments in the PSL score remained significant $P=0.036$, after Bonferroni adjustment). Only in the case of the increases in PSL, scores were positively correlated with the number of side effects $(r=0.838, P=0.009)$. Naltrexone alone did not elicit symptoms compatible with a positive challenge response in any of the participants across groups. Nonetheless, two female participants who were tested after one-hour pre-medication with naltrexone reported brief periods (less than a minute) of intense anxiety with symptoms compatible with a panic attack and a symptom-limited attack while they were away from the lab 5 to 6 hours after pre-medication. One participant experienced an episode of intense fear along with palpitations, sweating, dry mouth, difficulty breathing, hot flashes and fear of dying. The other subject also experienced intense fear, however, accompanied by sweating, trembling and fear of losing control. In both cases, symptoms were self-limited and resolved within a couple of hours.

After one-hour pre-medication the total number of reported side effects during the different conditions was not significantly different $(t=-0.565, d f=15, P=0.57)$. Conversely, the 
The effect of opioid receptor blockade on experimental panic provocation with $35 \% \mathrm{CO} 2$

group under five-hour pre-medication reported a higher number of adverse effects $(t=-2.990$, $d f=7, P=0.05$ ). The most common side effects attributed to naltrexone in this latter group were sleepiness, dizziness and loss of concentration.

Table 1. The effects of naltrexone premedication and a subsequent $\mathrm{CO} 2$ challenge on panic measures

\begin{tabular}{|c|c|c|c|c|c|c|c|}
\hline \multirow{2}{*}{\multicolumn{2}{|c|}{ Group/Measure }} & \multirow{2}{*}{ Condition } & \multicolumn{3}{|l|}{ Period } & \multirow{2}{*}{$\begin{array}{l}\begin{array}{l}\text { Naltrexone } \\
\text { alone }\end{array} \\
\text { Pre-CO2- } \\
\text { Baseline } \\
\end{array}$} & \multirow{2}{*}{$\begin{array}{l}\begin{array}{l}\mathrm{CO} 2 \\
\text { challenge }\end{array} \\
\text { Post- } \\
\text { Pre-CO2 }\end{array}$} \\
\hline & & & Baseline & Pre-CO2 & Post-CO2 & & \\
\hline \multirow{3}{*}{$\begin{array}{l}1 \text { hour pre- } \\
\text { medication } \\
(\mathrm{N}=16)\end{array}$} & PSL & $\begin{array}{l}\text { Placebo } \\
\text { Naltrexone }\end{array}$ & $\begin{array}{l}0.1(0.1) \\
0.3(0.1)\end{array}$ & $\begin{array}{l}0.7(0.2) \\
0.8(0.2)\end{array}$ & $\begin{array}{l}6.7(1.0) \\
9.2(1.8)\end{array}$ & $\begin{array}{l}0.5(0.2) \\
0.5(0.2)\end{array}$ & $\begin{array}{l}6.0( \pm 0.9) \\
8.3( \pm 1.7)\end{array}$ \\
\hline & VAS-A & $\begin{array}{l}\text { Placebo } \\
\text { Naltrexone }\end{array}$ & $\begin{array}{l}4.0(1.7) \\
4.8(3.1) \\
\end{array}$ & $\begin{array}{l}6.3(3.4) \\
9.2(4.4) \\
\end{array}$ & $\begin{array}{l}19.7(5.5) \\
22.5(6.2) \\
\end{array}$ & $\begin{array}{l}2.3(1.9) \\
4.3(1.5) \\
\end{array}$ & $\begin{array}{l}13.3( \pm 0.7) \\
13.3( \pm 3.0) \\
\end{array}$ \\
\hline & VAS-D & $\begin{array}{l}\text { Placebo } \\
\text { Naltrexone }\end{array}$ & $\begin{array}{l}4.7(2.3) \\
4.2(1.7)\end{array}$ & $\begin{array}{l}7.5(2.9) \\
13.7(4.4)\end{array}$ & $\begin{array}{l}36.0(6.2) \\
41.9(6.9)\end{array}$ & $\begin{array}{l}2.8(1.0) \\
9.5(3.3) \mathrm{a}\end{array}$ & $\begin{array}{l}28.5( \pm 4.7) \\
28.1( \pm 4.4)\end{array}$ \\
\hline \multirow{3}{*}{$\begin{array}{l}5 \text { hour pre- } \\
\text { medication } \\
(\mathrm{N}=8)\end{array}$} & PSL & $\begin{array}{l}\text { Placebo } \\
\text { Naltrexone }\end{array}$ & $\begin{array}{l}0.7(0.4) \\
0.3(0.1) \\
\end{array}$ & $\begin{array}{l}0.8(0.3) \\
2.5(0.6) \\
\end{array}$ & $\begin{array}{l}9.7(1.9) \\
11.6(2.0) \\
\end{array}$ & $\begin{array}{l}0.1(0.3) \\
2.1(0.6) a, c \\
\end{array}$ & $\begin{array}{l}8.8( \pm 1.8) \\
9.0( \pm 1.9) \\
\end{array}$ \\
\hline & VAS-A & $\begin{array}{l}\text { Placebo } \\
\text { Naltrexone } \\
\end{array}$ & $\begin{array}{l}2.2(1.1) \\
2.1(1.0) \\
\end{array}$ & $\begin{array}{l}7.0(2.4) \\
10.1(3.5) \\
\end{array}$ & $\begin{array}{l}33.8(11.6) \\
34.3(10.4) \\
\end{array}$ & $\begin{array}{l}4.7(2.4) \\
8.0(3.1) b \\
\end{array}$ & $\begin{array}{l}26.8( \pm 10.4) \\
24.2( \pm 9.5) \\
\end{array}$ \\
\hline & VAS-D & $\begin{array}{l}\text { Placebo } \\
\text { Naltrexone }\end{array}$ & $\begin{array}{l}4.3(2.3) \\
3.7(1.3)\end{array}$ & $\begin{array}{l}6.8(1.5) \\
16.2(4.1)\end{array}$ & $\begin{array}{l}43.6(10.8) \\
50.3(8.5)\end{array}$ & $\begin{array}{l}2.5( \pm 2.0) \\
12.5( \pm 4.2) \mathrm{a}\end{array}$ & $\begin{array}{l}33.3( \pm 8.0) \\
34.1( \pm 7.2)\end{array}$ \\
\hline
\end{tabular}

Mean scores $( \pm \mathrm{SE})$. "Paired $t$ tests (two-tailed) on main outcome measures, vs. Placebo: (a)Significant difference, (b)Marginally significant difference, (c)Significant difference after Bonferroni adjustment. PSL: DSM-IV Panic Symptoms List; VAS-A: Visual Analogue Scale-Anxiety; VAS-D: Visual Analogue Scale-Discomfort

\section{Discussion}

The findings on the effects of naltrexone alone, particularly the 5 -hour subgroup, suggests that exposure to prolonged opioid blockade can elicit a number of side effects and symptoms that somewhat mimic panic attacks. Furthermore, as noted in published case reports (Luby and Marrazzi 1987; Maremmani, Marini et al. 1998) and in two female subjects from our sample, some susceptible individuals may experience symptoms closely resembling spontaneous panic attacks from continued opioid blockade. Notwithstanding, opioid receptor blockade with $50 \mathrm{mg}$ of naltrexone did not modify the response to experimental panic provocation. The increases in all panic measurement scales after a double inhalation with $35 \% \mathrm{CO} 2$ were similar across naltrexone and placebo conditions. 
These results are in contrast to a previous report by Sinha et al (2007) where high doses of naloxone $(2 \mathrm{mg} / \mathrm{kg})$ increased the subjective and ventilatory response to a racemic lactate infusion in healthy volunteers. Aside from the fact that we did not measure objective respiratory values, a number of methodological differences may account for our findings. For instance, whereas a racemic lactate infusion usually elicits a response in a few, if any, healthy subjects (Liebowitz, Fyer et al. 1984), a double inhalation with 35\% produces significant increases in panic measurement and the percentage of "responders" (Griez, Colasanti et al. 2007). This increased response may have obscured a modest effect produced by opioid blockade. Another important divergence may be the dosage as well as the pharmacodynamic and pharmacokinetic properties of the different administered opioid blockers, which make comparisons difficult. An intravenous dose of $2 \mathrm{mg} / \mathrm{kg}$ of naloxone may provide full receptor blockade and elicit many panic-like symptoms and hyperventilation (Cohen, Cohen et al. 1983). On the other hand, orally administered naltrexone can have a bioavailability of up to $40 \%$, is at least twice as potent as naloxone and has a half-life of 14 hours (Gonzalez and Brogden 1988). As such, in a estimate given a median subject weight of $61 \mathrm{~kg}$ in our sample, an oral administration of $50 \mathrm{mg}$ of naltrexone can be roughly equivalent to the lowest dose of naloxone used $(0.5 \mathrm{mg} / \mathrm{kg})$ in the study conducted by Sinha et al (2007).

The long half-life of oral opioid blockers such as naltrexone may be relevant as the influence of opioid receptor blockade can be time dependent due to displacement and receptor occupancy kinetics (Yassen, Olofsen et al. 2007). This is in-line with the findings described earlier in case reports and the two female subjects in our 1-hour test group, where spontaneous panic attacks were precipitated a few hours after the administration of an oral opioid receptor blocker. Accordingly, we also tested the vulnerability to $\mathrm{CO} 2$ in a subgroup of 8 females who were exposed to 5 hours of opioid blockade but found no increase in their reactions to experimental panic provocation.

Several caveats, such as the small sample size, the use of healthy volunteers and no comparison group including patients with panic disorder, must be taken into account when interpreting our data. These limitations aside, results from the present study suggest that prolonged exposure to opioid blockade itself can elicit symptoms that resemble panic. As much as these effects can potentially explain the findings of those reports that link panic attacks with the opioid system, they do not seem to be related to the mechanisms that underlie $\mathrm{CO} 2$ induced panic. Admittedly, a weaker panic stimulation and/or a higher concentration of opioid blocker may be required to elicit an increase in the susceptibility to panicogenic maneuvers. Further studies are required to better determine the role of opioids in panic. 
The effect of opioid receptor blockade on experimental panic provocation with $35 \% \mathrm{CO} 2$

\section{References}

Akiyama, Y., M. Nishimura, et al. (1993). "Effects of naloxone on the sensation of dyspnea during acute respiratory stress in normal adults." 1 Appl Physiol 74(2): 590-5.

Battaglia, M. and G. Perna (1995). "The 35\% CO2 challenge in panic disorder: optimization by receiver operating characteristic (ROC) analysis." J Psychiatr Res 29(2): 111-9.

Cohen, M. R., R. M. Cohen, et al. (1983). "High-dose naloxone infusions in normals. Dose-dependent behavioral, hormonal, and physiological responses." Arch Gen Psychiatry 40(6): 613-9.

Gonzalez, J. P. and R. N. Brogden (1988). "Naltrexone. A review of its pharmacodynamic and pharmacokinetic properties and therapeutic efficacy in the management of opioid dependence." Drugs 35(3): 192-213.

Griez, E. J., A. Colasanti, et al. (2007). "Carbon dioxide inhalation induces dose-dependent and agerelated negative affectivity." PLOS ONE 2(10): e987.

Jennings, A. L., A. N. Davies, et al. (2002). "A systematic review of the use of opioids in the management of dyspnoea." Thorax 57(11): 939-44.

Liebowitz, M. R., A. J. Fyer, et al. (1984). "Lactate provocation of panic attacks. I. Clinical and behavioral findings." Arch Gen Psychiatry 41 (8): 764-70.

Liebowitz, M. R., J. M. Gorman, et al. (1984). "Effects of naloxone on patients with panic attacks." Am J Psychiatry 141(8): 995-7.

Luby, E. D. and M. A. Marrazzi (1987). "A panic attack precipitated by opiate blockade--a case study." $\underline{\mathrm{J}}$ Clin Psychopharmacol 7 (5): 361-2.

Maremmani, I., G. Marini, et al. (1998). "Naltrexone-induced panic attacks." Am J Psychiatry 155(3): 447.

Poma, S. Z., S. Milleri, et al. (2005). "Characterization of a 7\% carbon dioxide (CO2) inhalation paradigm to evoke anxiety symptoms in healthy subjects." J Psychopharmacol 19(5): 494-503.

Preter, M. and D. F. Klein (2008). "Panic, suffocation false alarms, separation anxiety and endogenous opioids." Prog Neuropsychopharmacol Biol Psychiatry 32(3): 603-12.

Sheehan, D. V., Y. Lecrubier, et al. (1998). "The Mini-International Neuropsychiatric Interview (M.I.N.I.): the development and validation of a structured diagnostic psychiatric interview for DSM-IV and ICD10." J Clin Psychiatry 59 Suppl 20: 22-33;quiz 34-57.

Sinha, S. S., R. R. Goetz, et al. (2007). "Physiological and behavioral effects of naloxone and lactate in normal volunteers with relevance to the pathophysiology of panic disorder." Psychiatry Res 149(1-3): 309-14.

Yassen, A., E. Olofsen, et al. (2007). "Mechanism-based pharmacokinetic-pharmacodynamic modelling of the reversal of buprenorphine-induced respiratory depression by naloxone: a study in healthy volunteers." Clin Pharmacokinet 46(11): 965-80. 
Chapter 6:

\title{
The association between 5-HTTLPR genotype and $\mathrm{CO} 2$ induced negative affectivity
}

\author{
Schruers $K^{1,2}$, Esquivel $G^{1,2}$, van Duinen $M^{1,2}$, Wichers $M^{2}$, Kenis $G^{2}$, \\ Colasanti $A^{1,2}$, Knuts $1^{1,2}$, Goossens $L^{1,2}$, Jacobs $N^{2}$, van Rozendaal $J^{2}$, \\ van $O s \mathrm{~J}^{2}$, and Griez $\mathrm{E}^{1,2}$ \\ ${ }^{1}$ Academic Anxiety Center and ${ }^{2}$ School for Mental Health and Neurosciences, \\ Faculty of Medicine, Health and Life Sciences, Maastricht University, \\ Maastricht, The Netherlands
}


The association between 5-HTTLPR genotype and $\mathrm{CO} 2$ induced negative affectivity

\begin{abstract}
Background: Inhalation of an increased concentration of carbon dioxide (CO2) has been shown to induce a state of negative affect in healthy subjects that is closely related to the clinical phenomenon of panic. It has been suggested that the vulnerability to $\mathrm{CO}_{2}$ is moderated by differences in serotonin $(5-H T)$ activity, caused by a functional polymorphism in the promoter region of the 5-HT transporter gene (5-HTTLPR). Our aim was to examine the relationship between 5 -HTTLPR genotype and the affective response to different dosages of inhaled $\mathrm{CO} 2$ in healthy volunteers.

Methods: Ninety-six subjects performed a double-inhalation of four mixtures containing respectively $0,9,17.5$ and $35 \% \mathrm{CO} 2$, following a double-blind, cross-over, randomized design. Affective responses were measured with a visual analogue scale for fear and the Panic Symptom List. 5-HTTLPR genotype was expressed as LL, SL and SS.

Results: Subjects with the SL and SS genotype reported less fear than LL subjects. A significant interaction effect was found between genotype and $\mathrm{CO} 2$ dosage: the SS genotype showed lower fear scores than the LL genotype, particularly in the $17.5 \% \mathrm{CO} 2$ dose condition. Conclusions: The present study suggests that the dose-dependent fear reaction to $\mathrm{CO} 2$ is moderated by a polymorphism in the $5-\mathrm{HT}$ transporter gene, particularly at intermediate $\mathrm{CO} 2$ dosages. It also underscores the usefulness of the introduction of an experimental environmental factor to reveal underlying vulnerability in a phenotype, otherwise staying elusive. These results are in line with current theories on the role of 5-HT in both panic and respiration.
\end{abstract}

Key words: carbon dioxide, panic disorder, anxiety, 5-HT transporter gene. 
Introduction

Panic attacks are a common phenomenon, given the fact that $20 \%$ of people in the general population experience a panic attack once in their lives (Kessler, Chiu et al. 2006). A number of them go on to develop panic disorder, which also includes anticipatory anxiety and avoidance behavior, next to the repetitive occurrence of panic attacks (American Psychiatric Association 2000). Panic disorder (PD) is highly prevalent, occurring in $4-6 \%$ of patients in primary care, and is associated with multiple medically unexplained symptoms as well as high medical costs (Katon, Roy-Byrne et al. 2002). The underlying pathophysiology of panic remains unknown. We have shown that panic can be reliably evoked in healthy volunteers (Griez, Colasanti et al. 2007). Inhalation of increasing concentrations of carbon dioxide (CO2) dose-dependently induces a negative affect in healthy volunteers, complying with formal criteria of panic, as defined in current psychiatric nosology. This experimental paradigm opens the unique possibility of studying the underlying biology of a psychopathological phenomenon in the laboratory. If the reaction of healthy volunteers to $\mathrm{CO} 2$ inhalation is indeed comparable to real life panic in PD patients, the factors that moderate the occurrence of panic attacks in patients should also influence the experimental phenotype.

There is a large body of evidence regarding the role of serotonin (5-hydroxytryptamin, $5-H T 7$ in panic, both in the laboratory and in natural circumstances. Challenge studies, involving the experimental manipulation of $5-\mathrm{HT}$ availability to the brain are informative to elucidate the role of 5-HT in panic, especially when combined with the use of a panic challenge. Brain 5-HT levels can be substantially decreased by tryptophan depletion (TD). Several studies showed that the reaction to an experimental panic challenge increased in PD patients, when undergoing TD (Kent, Coplan et al. 1996; Miller, Deakin et al. 2000; Schruers, Klaassen et al. 2000). Conversely, increasing 5-HT availability decreased the panic response (Schruers, van Diest et al. 2002; Maron, Toru et al. 2004; Schruers and Griez 2004). Similarly, metergoline (a 5-HT2A/2Cand 5-HT1B/D receptor antagonist) increased the reaction to a CO2 panic challenge (Ben-Zion, Meiri et al. 1999), while d-fenfluramine (a 5-HT releasing agent) was shown to have an inhibitory effect (Mortimore and Anderson 2000).

Synaptic serotonin function relies on a number of proteins. The 5-HT transporter (5$\mathrm{HTT}$ is located on the presynaptic neuron and eliminates $5-\mathrm{HT}$ from the synaptic cleft. The main catabolic pathway is oxidative deamination by Monoamine Oxidase-A (MAO-A). $5-H T$ is synthesized from tryptophan, an essential amino acid. The rate-limiting step in this process is controlled by tryptophan hydroxylase. Many 5-HT receptors have also been identified, some of whom are proposed as playing a role in panic: 5-HT1A, 5-HT1B, 5-HT2A and 5-HT2C (Graeff, Viana et al. 1997; Graeff and Del-Ben 2008). Several functional polymorphisms in the genes for these proteins have been described and many association studies examining the 
The association between 5-HTTLPR genotype and $\mathrm{CO} 2$ induced negative affectivity

contribution of these polymorphisms have been carried out. Results of those studies have been inconsistent and replication of findings has proven to be difficult (for an extensive recent review see (Maron and Shlik 2006).

A particularly well studied example is a functional length polymorphism (termed 5HTTLPR, serotonin transporter-linked polymorphic region) in the promoter region of the 5-HTT (or SLC6A4) gene, which was first described by Lesch and colleagues (Lesch, Bengel et al. 1996 ) in association with anxiety related personality traits. In panic disorder, results of association studies with 5-HTTLPR were also mostly inconclusive (Blaya, Salum et al. 2007; Strug, Suresh et al. 2008). However, this may be due to how the underlying genetic hypothesis was formulated and which methodology was applied to test it. Genetic association studies assume a direct linear relation between genes and a disorder. However, such relationships are rare in psychiatric disorders. Gene-environment studies, on the other hand, assume that environmental pathogens are on the causal pathway to a certain disorder and that genes moderate susceptibility to such pathogens (Caspi and Moffitt 2006). Given the fact that the genetic contribution to the liability of panic disorder is around $30-40 \%$, a major role for environmental factors may be inferred (Hettema, Neale et al. 2001; Hettema, Prescott et al. 2005). Therefore, testing for genetic moderation in genetic association studies may not be powerful if there is underlying gene-environment interaction. Enriching genetic studies with environmental information may yield conclusive results in the case of gene-environment interaction, as genetic main effects may remain under the threshold of detection Nan Os and Sham 2003). Carbon dioxide is an environmental factor capable of inducing panic reactions, which can be moderated by manipulation of 5-HT activity (Schruers, Klaassen et al. 2000; Schruers, van Diest et al. 2002; Schruers and Griez 2004). Previous studies examining the influence of 5-HTTLPR on the effects of $\mathrm{CO} 2$ exposure have yielded interesting results. A study in healthy volunteers showed that the LL genotype (homozygous for the L-allele) predicted a greater fearful response to a single 35\% CO2 inhalation (Schmidt, Storey et al. 2000). Although this result could not be replicated in PD patients (Perna, di Bella et al. 2004), the same researchers demonstrated that the presence of the L-allelic variant was associated with a better therapeutic response to the serotonin reuptake inhibiting drug paroxetine in PD (Perna, Favaron et al. 2005).

We previously proposed that the dose dependent negative affect induced by $\mathrm{CO} 2$ is closely related to the panic experienced by PD patients (Griez, Colasanti et al. 2007). If this is indeed the case, the hypothesis that experimental induction of panic may be moderated by the same functional polymorphism in the 5-HT transporter gene suggests itself. Therefore, the present study examined the interaction between 5-HTTLPR and four differently dosed $\mathrm{CO} 2$ challenges in a group of healthy volunteers. 


\section{Methods}

\section{Subjects}

Ninety-six volunteers participated in the study, consisting of 30 males and 66 females, aged $29.2(S D=10.2)$ and $26.2(S D=9.7)$ years respectively.

All potential participants had a complete inventory of medical history and a physical examination. Inclusion criteria were being 18 to 65 years of age and having good present and past physical and mental condition. Mental condition was assessed by a structured psychiatric interview (Mini International Neuropsychiatric Interview) performed by an experienced psychiatrist or psychologist. Exclusion criteria included a history of pulmonary or cardiovascular disease, the presence of hypertension (diastolic $>100 \mathrm{mmHg}$; systolic $>170$ $\mathrm{mmHg}$ ), cerebral aneurysm, pregnancy, epilepsy, excessive smoking (>15 cigarettes/day), use of adrenergic receptor blockers and use of psychotropic medication. Participants with any history of psychiatric disorder, as assessed by MINI or anamnestic information, were excluded from participation. The Medical Ethics Committee of Maastricht University and Maastricht University Hospital approved the study.

\section{Procedure}

The inhalation apparatus and the general procedures used in our laboratory have been described in detail elsewhere (Verburg, Perna et al. 2001).

The procedure for each subject consisted in a double inhalation of four mixtures containing respectively $0,9,17.5$ and $35 \% \mathrm{CO} 2$ in air, following a double blind randomized design.

Subjects were instructed in the use of a mask with a demand valve for selfadministration of medical gasses and told that they would take a double vital capacity breath of four different concentrations of $\mathrm{CO} 2$ in air, which, though being a harmless physiological substance might cause brief neurovegetative responses and arousal or fear, depending on concentration. Subjects were asked to exhale to the maximum, to position the inhalation mask on their face and inhale their full capacity as quickly as possible. Next they were to empty their lungs and refill them immediately with the gas mixture, whereupon they had to hold their breath for 4 seconds before exhaling. All inhalations took place within one week, on four separate days but at the same time of day for each participant. Care was taken that each inhalation represented at least $80 \%$ of the subject's vital capacity.

\section{Assessment}

Affective responses were assessed with reference to the DSM N (American Psychiatric Association 2000), which refers to a panic attack as "a discrete period of intense fear or 
The association between 5-HTTLPR genotype and $\mathrm{CO} 2$ induced negative affectivity

discomfort", in which four (or more) out of a list of thirteen predefined symptoms develop abruptly and reach a peak within 10 minutes.

In accordance with this description, a visual analogue scale for fear (VAS-F) was used. The scale was anchored from 0 , "no fear at all", to 100, "the worst imaginable fear". This instrument has been validated previously for use during 35\% CO2 challenges (van Duinen, Rickelt et al. 2008).

The Panic Symptom List (PSL-IV) was used to evaluate panic symptomatology (Schruers, Klaassen et al. 2000). PSL-IV is a questionnaire listing thirteen items, each item representing a DSM-IV panic symptom, to be rated on a five-point scale, from 0 (absent) to 4 (very intense). The PSL-IV and VAS-F were administered one minute before and after each inhalation. Delta VAS-F and PSL scores were calculated for each assessment.

\section{Genotyping}

Buccal cell material was sampled from all participants, using Whatman's Sterile Omni Swabs (www.whatman.com), specifically designed for collection of buccal cell samples for DNA testing.

Genomic DNA was isolated using the QIAamp DNA Mini Kit from Qiagen Nenlo, The Netherlands). Determination of the 5-HTTLPR genotype was performed as previously described (Park, Kim et al. 2004), with some modifications. The forward primer was FAMlabeled and had the sequence (5'-GGCGTTGCCGCTCTGAATGC-3'), and the reverse (5'GAGGGACTGAGCTGGACAACCCAC-3'). Polymerase chain reactions were carried out in 96well microtiterplates on a Biometra T1 Thermocycler (Westburg, Leusden, The Netherlands). We used approximately 10-100 ng of genomic DNA in a $25 \mu \mathrm{l}$ reaction mixture containing $1 \mathrm{x}$ PCR buffer (Invitrogen, Breda, The Netherlands), $0.2 \mathrm{mM}$ dNTPs, $0.4 \mu \mathrm{M}$ of each primer, 0.75 $\mathrm{mM} \mathrm{MgCl2}$, and $1 \mathrm{U}$ Taq DNA polymerase (Invitrogen, Breda, The Netherlands). Cycling conditions were: initial $3 \mathrm{~min}$. denaturation at $95^{\circ} \mathrm{C} ; 5$ cycles of denaturation at $94^{\circ} \mathrm{C}$ for 30 sec., annealing at $65^{\circ} \mathrm{C}$ (touch down $0.3^{\circ} \mathrm{C}$ ) for $1 \mathrm{~min}$. and extension at $72^{\circ} \mathrm{C}$ for 1 min.; 30 cycles of denaturation at $94^{\circ} \mathrm{C}$ for $30 \mathrm{sec}$, annealing at $63^{\circ} \mathrm{C}$ for 1 min. and extension at $72^{\circ} \mathrm{C}$ for $1 \mathrm{~min}$;; and a final extension for $10 \mathrm{~min}$. at $72^{\circ} \mathrm{C}$. PCR products were size-resolved on a $\mathrm{AB}$ | 3100 Genetic Analyzer and analyzed using Genescan Analysis software (Applied Biosystems, Nieuwerkerk a/d IJssel, The Netherlands).

In the analyses, 5-HTTLPR genotype was expressed as: $1=$ two long alleles (LL); 2=one short and one long allele (SL); 3=two short alleles (SS). 


\section{Statistical analysis}

Multilevel linear regression analyses, using the XTREG command in STATA 10 (StataCorp, 2007), were applied to the data in order to account for the clustering of CO2 levels (level 1) within participants (level 2) (Snijders and Bosker 1999).

First, changes in subjective fear (VAS-F) and physical symptoms (PSL) during $\mathrm{CO} 2$ exposure were regressed on 5-HTTLPR genotype. Second, changes in subjective fear and physical symptoms were regressed on the interaction between 5-HTTLPR genotype and $\mathrm{CO} 2$ dose. Effect sizes were calculated for fear scores for each genotype separately by applying and testing the appropriate linear combinations using the STATA LINCOM command. Main effects and interactions were assessed by Wald test.

\section{Results}

Effect of 5-HTTLPR genotype on fear during $\mathrm{CO} 2$ exposure

A significant main effect was present of genotype on development of fear during $\mathrm{CO} 2$ exposure. Both subjects with the $S L$ genotype $(B=-6.6, p=0.023)$ and $S S$ genotype $(B=-7.0$, $p=0.045$ ) reported on average less fear than $L L$ subjects (see figure 1 for fear scores by genotype). A significant genotype $\times \mathrm{CO} 2$ dosage interaction effect was apparent $(x=3.89$, $p=0.048$ ). Analyzing CO2 dosage as a continuous exposure showed that the SS genotype differed significantly from the $L L$ genotype in fear score patterns along an increased dosage of $\mathrm{CO} 2$ exposure $(B=-3.78)$. Analyzing $\mathrm{CO} 2$ exposure as a factored variable using $0 \%$ as the reference value suggested maximum separation of response by genotype in the $17.5 \% \mathrm{CO} 2$ dose condition $(x=6.54, p=0.009, B=-14.94)$. The other $\mathrm{CO} 2$ doses showed directionally similar but non-significant separation by genotype (Figure 2, table 1 for the full overview of fear scores for each $\mathrm{CO} 2$ dosage per genotype and for interactions tested).

Figure 1. Average fear score by genotype

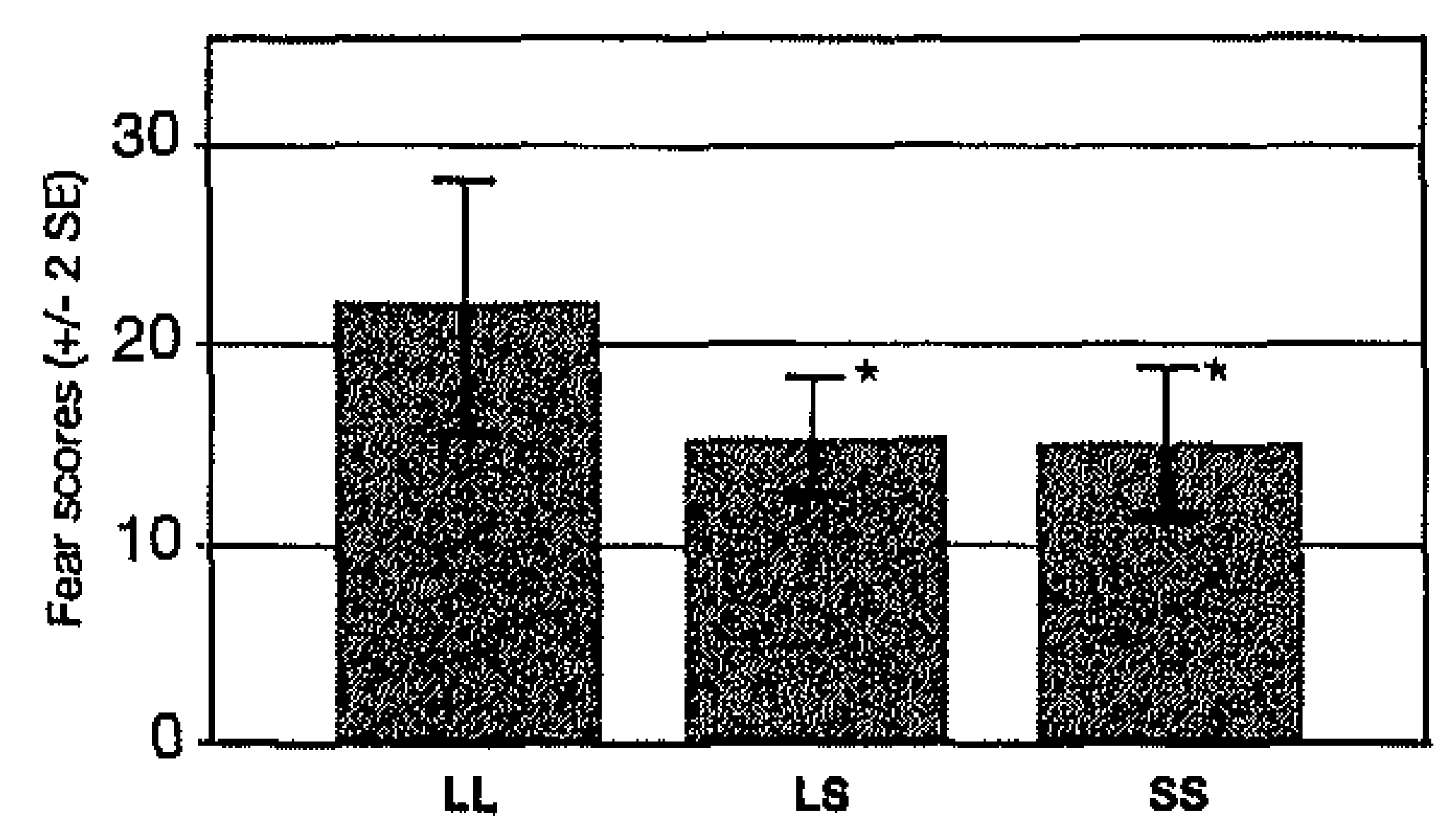

*Difference compared with $L L$ genotype $(P<.05)$ 
The association between 5-HTTLPR genotype and $\mathrm{CO} 2$ induced negative affectivity

Effect of 5-HTTLPR genotype on panic symptoms during CO2 exposure

There was no main effect of genotype on symptom scores ( $S L$ compared to LL genotype: $B=-$ $0.02, p=0.9$; SS compared to LL genotype: $B=0.41, p=0.6$ ). In addition, no interaction was found between increased dosage of $\mathrm{CO} 2$ and genotype on symptoms (LS compared to LL genotype: $\chi=1.15, p=0.3$ and SS compared to LL genotype: $\chi=0.14, p=0.7$ ).

Figure 2. Effect of 5-HTTLPR genotype on fear during $\mathrm{CO} 2$ exposure
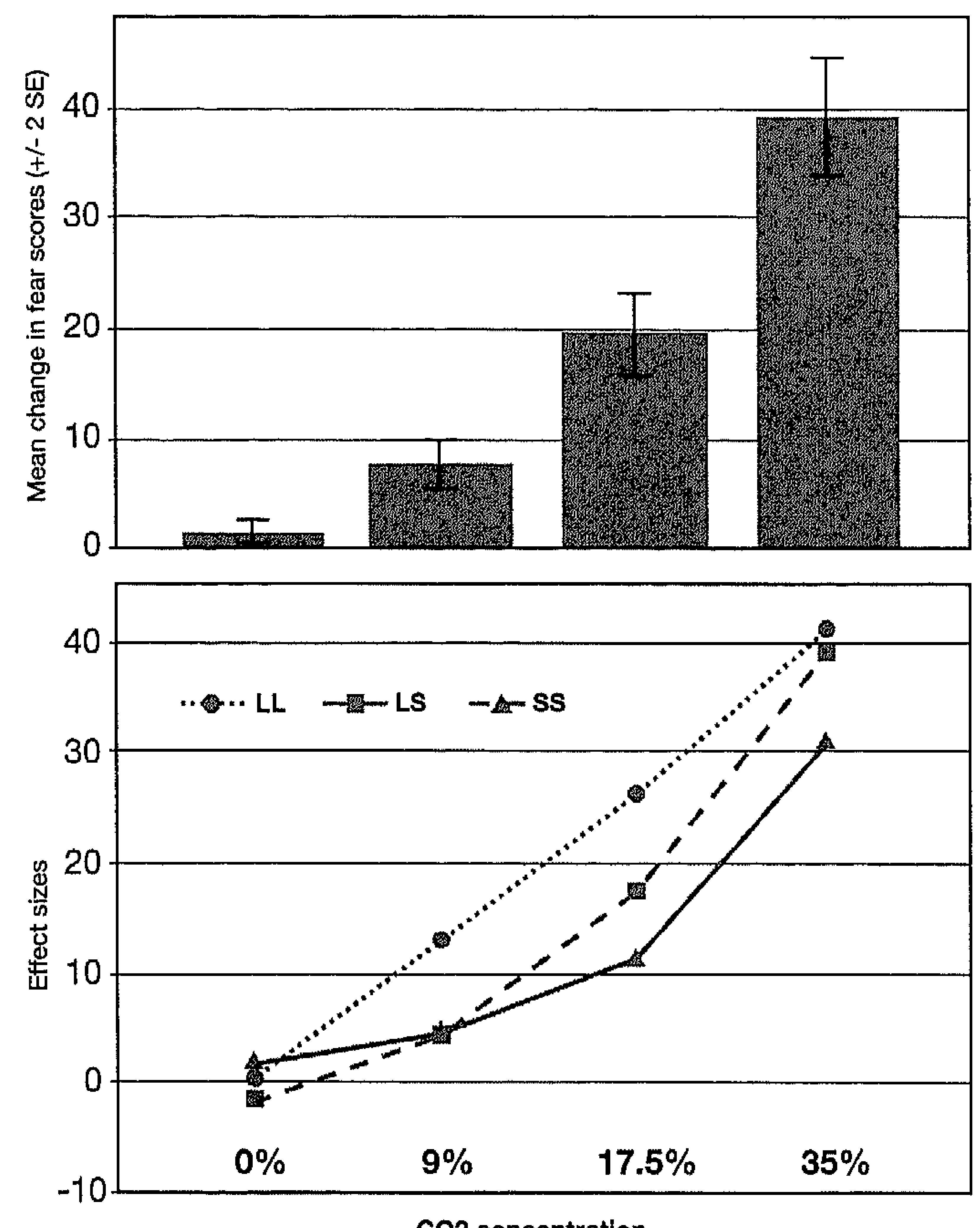

CO2 concentration

5-HTTLPR genotype: $L L=t w o$ long alleles; $S L=o n e$ short and one long allele; $S S=t w o$ short alleles 
Chapter 6

Table 1. Testing the interaction with genotype on fear score per dosage of $\mathrm{CO} 2$

\begin{tabular}{lllll}
\hline Interaction & Coefficient & $P$ & \multicolumn{3}{l}{$95 \%$ Conf. Interval } \\
\hline LS $\cdot 9 \%$ & -8.811012 & 0.065 & -18.18386 & -.5618356 \\
LS $\cdot 17.5 \%$ & -8.975368 & 0.061 & -18.36405 & -.4133178 \\
LS $\cdot 35 \%$ & -2.202401 & 0.645 & -11.57525 & -7.170446 \\
\hline SS $\cdot 9 \%$ & -8.622944 & 0.134 & -19.8976 & -2.651716 \\
SS $\cdot 17.5 \%$ & -14.93593 & 0.009 & -26.21059 & -3.661271 \\
SS $\cdot 35 \%$ & -10.49351 & 0.068 & -21.76817 & -.7811532 \\
\hline
\end{tabular}

LS and SS vs. LL

Discussion

The present study demonstrates that the dose-dependent fear reaction to $\mathrm{CO} 2$ administration is moderated by a polymorphism in the $5-H T$ transporter gene. More specifically, individuals with the LL genotype displayed a stronger fear reaction to $\mathrm{CO} 2$, particularly at intermediate dosages. These results are in accordance with those of a previous study using only one single 35\% CO2 administration in healthy volunteers (Schmidt, Storey et al. 2000). In this study, the LL allele was similarly associated with stronger reactivity to the challenge. However, this result was not replicated in a subsequent study in PD patients (Perna, di Bella et al. 2004). This seemingly contradictory result may be explained by taking into account the dose-response relationship between $\mathrm{CO} 2$ dosage and fear reaction. Thus, evidence for genetic moderation in the present study was strongest in the double $17.5 \%$ condition, which is roughly equivalent to the single $35 \%$ used in the Schmidt et al study. At the highest dosage of double $35 \%$, no significant interaction occurred, although the effect was directionally similar and only somewhat weaker. We have previously shown that double inhalations of increasingly higher concentrations of $\mathrm{CO} 2$ induce a reaction in healthy volunteers that is identical to the panic response in PD patients, particularly at the highest dosage of double breath $35 \% \mathrm{CO} 2$ (Griez, Colasanti et al. 2007). Therefore, regarding the effect, this double breath $35 \% \mathrm{CO} 2$ condition in healthy volunteers appears to be comparable to the single breath $35 \% \mathrm{CO} 2$ in the study by Perna and colleagues in PD patients (Perna, di Bella et al. 2004). Thus, at this particularly elevated dose or in particularly vulnerable panic disorder patients, the administered stimulus is so powerful that genetic moderation cannot be detected due to a ceiling effect.

Taken together, present and previous results suggest a link between 5-HT function, CO2 sensitivity and panic (Schruers, Klaassen et al. 2000; Schruers, van Diest et al. 2002; Schruers and Griez 2004; Griez, Colasanti et al. 2007). The nature of this link may be found in the recently described role of medullary 5 -HT neurons as chemosensors, sensitive to CO2 via 
The association between 5-HTTLPR genotype and $\mathrm{CO} 2$ induced negative affectivity

changes in $\mathrm{pH}$. These neurons have no direct role in control of breathing, however. On the contrary, they project rostrally towards the forebrain and are proposed to have a function in arousal, sleep, cerebrovascular tone and panic, with a common aim to restore $\mathrm{pH}$ homeostasis (Severson, Wang et al. 2003; Richerson 2004).

In most studies examining the effect of 5-HTTLPR on vulnerability to depression, presence of the S-allele is associated with increased vulnerability for a recent review and meta-analysis, see (Munafo, Durrant et al. 2008)). The situation may be different in situations of natural or experimentally induced tryptophan depletion (Moreno, Rowe et al. 2002; Neumeister, Hu et al. 2006; Sanjuan, Martin-Santos et al. 2008). However, in the studies examining the effects of $\mathrm{CO} 2$, it is the L-allele that is associated with increased vulnerability (present study and (Schmidt, Storey et al. 2000)). Serotonin fulfills a wide range of functions in the body and these data indicate that increased or decreased availability can be advantageous or disadvantageous, depending on the situation. As far as the neurobiology of panic and anxiety is concerned, this is accounted for by a hypothesis put forward by Deakin and Graeff (Graeff and Del-Ben 2008). It states that 5-HT enhances anxiety in the forebrain, while inhibiting panic in the midbrain peri-aqueductal gray.

Two arguments suggest that the present results may be relevant. First, the results yield more insight into the modulatory role of 5-HT in panic. Secondly, they demonstrate that the experimental provocation of panic in healthy volunteers is sensitive to subtle influences such as length variations in a single gene. This is promising as it opens the possibility for early stage testing of anti-panic drugs, without the need to sample PD patients.

In conclusion, the present study replicates and extends previous work regarding the behavioral effects of increasing dosages of $\mathrm{CO} 2$ in a larger, independent sample (Griez, Colasanti et al. 2007). The current findings suggest that a polymorphism in the promoter region of the 5-HT transporter gene moderates the dose-dependent fear reaction to CO2. These results are in line with current theories on the role of $5-\mathrm{HT}$ in the regulation of both panic and respiration. 


\section{References}

American Psychiatric Association (2000). Diagnostic and statistical manual of mental disorders: DSM-IVTR. Washington, DC, American Psychiatric Association.

Ben-Zion, I. Z., G. Meiri, et al. (1999). "Enhancement of CO2-induced anxiety in healthy volunteers with the serotonin antagonist metergoline." Am J Psychiatry 156(10): 1635-7.

Blaya, C., G. A. Salum, et al. (2007). "Lack of association between the Serotonin Transporter Promoter Polymorphism (5-HTTLPR) and Panic Disorder: a systematic review and meta-analysis." Behav Brain Funct 3: 41.

Caspi, A. and T. E. Moffitt (2006). "Gene-environment interactions in psychiatry: joining forces with neuroscience." Nat Rev Neurosci 7 (7): 583-90.

Graeff, F. G. and C. M. Del-Ben (2008). "Neurobiology of panic disorder: From animal models to brain neuroimaging." Neurosci Biobehav Rev 32(7): 1326-35.

Graeff, F. G., M. B. Viana, et al. (1997). "Dual role of 5-HT in defense and anxiety." Neurosci Biobehav Rev 21(6): 791-9.

Griez, E. J., A. Colasanti, et al. (2007). "Carbon dioxide inhalation induces dose-dependent and agerelated negative affectivity." PLOS ONE 2(10): e987.

Hettema, J. M., M. C. Neale, et al. (2001). "A review and meta-analysis of the genetic epidemiology of anxiety disorders." Am J Psychiatry 158(10): 1568-78.

Hettema, J. M., C. A. Prescott, et al. (2005). "The structure of genetic and environmental risk factors for anxiety disorders in men and women." Arch Gen Psychiatry 62(2): 182-9.

Katon, W. J., P. Roy-Byrne, et al. (2002). "Cost-effectiveness and cost offset of a collaborative care intervention for primary care patients with panic disorder." Arch Gen Psychiatry 59(12): 1098104.

Kent, J. M., J. D. Coplan, et al. (1996). "Ventilatory effects of tryptophan depletion in panic disorder: a preliminary report." Psychiatry Res 64(2): 83-90.

Kessler, R. C., W. T. Chiu, et al. (2006). "The epidemiology of panic attacks, panic disorder, and agoraphobia in the National Comorbidity Survey Replication." Arch Gen Psychiatry 63(4): 41524.

Lesch, K. P., D. Bengel, et al. (1996). "Association of anxiety-related traits with a polymorphism in the serotonin transporter gene regulatory region." Science 274(5292): 1527-31.

Maron, E. and J. Shlik (2006). "Serotonin function in panic disorder: important, but why?" Neuropsychopharmacology 31(1): 1-11.

Maron, E., I. Toru, et al. (2004). "The effect of 5-hydroxytryptophan on cholecystokinin-4-induced panic attacks in healthy volunteers." J Psychopharmacol 18(2): 194-9.

Miller, H. E., J. F. Deakin, et al. (2000). "Effect of acute tryptophan depletion on CO2-induced anxiety in patients with panic disorder and normal volunteers." Br J Psychiatry 176: 182-8.

Moreno, F. A., D. C. Rowe, et al, (2002). "Association between a serotonin transporter promoter region polymorphism and mood response during tryptophan depletion." Mol Psychiatry 7(2): 213-6.

Mortimore, C. and I. M. Anderson (2000). "d-Fenfluramine in panic disorder: a dual role for 5hydroxytryptamine." Psychopharmacology (Berl) 149(3): 251-8.

Munafo, M. R., C. Durrant, et al. (2008). "Gene x Environment Interactions at the Serotonin Transporter Locus." Biol Psychiatry.

Neumeister, A., X. Z. Hu, et al. (2006). "Differential effects of 5-HTTLPR genotypes on the behavioral and neural responses to tryptophan depletion in patients with major depression and controls." Arch Gen Psychiatry 63(9): 978-86.

Park, J. W., J. S. Kim, et al. (2004). "Serotonin transporter polymorphism and harm avoidance personality in chronic tension-type headache." Headache 44(10): 1005-9.

Perna, G., D. di Bella, et al. (2004). "Lack of relationship between CO2 reactivity and serotonin transporter gene regulatory region polymorphism in panic disorder." Am J Med Genet B Neuropsychiatr Genet 129B(1): 41-3

Perna, G., E. Favaron, et al. (2005). "Antipanic efficacy of paroxetine and polymorphism within the promoter of the serotonin transporter gene." Neuropsychopharmacology 30(12): 2230-5. 
The association between 5-HTTLPR genotype and $\mathrm{CO} 2$ induced negative affectivity

Richerson, G. B. (2004). "Serotonergic neurons as carbon dioxide sensors that maintain pH homeostasis." Nat Rev Neurosci 5(6): 449-61.

Sanjuan, J., R. Martin-Santos, et al. (2008). "Mood changes after delivery: role of the serotonin transporter gene." Br J Psychiatry 193(5): 383-8.

Schmidt, N. B., J. Storey, et al. (2000). "Evaluating gene x psychological risk factor effects in the pathogenesis of anxiety: a new model approach." J Abnorm Psychol 109(2): 308-20.

Schruers, K. and E. Griez (2004). "The effects of tianeptine or paroxetine on $35 \% \mathrm{CO} 2$ provoked panic in panic disorder." J Psychopharmacol 18(4): 553-8.

Schruers, K., T. Klaassen, et al. (2000). "Effects of tryptophan depletion on carbon dioxide provoked panic in panic disorder patients." Psychiatry Res 93(3): 179-87.

Schruers, K., R. van Diest, et al. (2002). "Acute L-5-hydroxytryptophan administration inhibits carbon dioxide-induced panic in panic disorder patients." Psychiatry Res 113(3): 237-43.

Severson, C. A., W. Wang, et al. (2003). "Midbrain serotonergic neurons are central pH chemoreceptors." Nat Neurosci 6(11): 1139-40.

Snijders, T. A. B. and R. J. Bosker (1999). Multilevel analysis: An introduction to basic and advanced multilevel modeling. London, Sage Publishers.

Strug, L. J., R. Suresh, et al. (2008). "Panic disorder is associated with the serotonin transporter gene (SLC6A4) but not the promoter region (5-HTTLPR)." Mol Psychiatry.

van Duinen, M., J. Rickelt, et al. (2008). "Validation of the electronic Visual Analogue Scale of Anxiety." Prog Neuropsychopharmacol Biol Psychiatry 32(4): 1045-7.

Van Os, J. and P. Sham (2003). Gene-environment interactlons. The Epidemiology of Schizophrenia. R. M. Murray, P. B. Jones, E. Susser, J. Van Os and M. Cannon. Cambridge, Cambridge University Press: pp. 235-254.

Verburg, K., G. Perna, et al. (2001). A case study of the $35 \%$ CO2 challenge. Anxiety Disorders. An Introduction to Clinical Management and Research. E. J. L. Griez, C. Faravelli, D. Nutt and J. Zohar. Chichester, Wiley: 341-357. 


\section{Chapter 7:}

The relationship between habitual physical activity and symptom severity in panic disorder

Esquivel G, Schruers K, Colasanti A, Pereboom M, Van Diest R and Griez E

School for Mental Health and Neurosciences, Maastricht University; and Academic Anxiety Center Maastricht, Mondriaan Zorggroep, Maastricht, The Netherlands 
The relationship between habitual physical activity and symptom severity in panic disorder

\begin{abstract}
Objective: Very few studies have addressed the potential benefits of aerobic exercise in patients with panic disorder. There is not only a need to demonstrate the efficacy of exercise treatment but also to characterize the way it may have an effect on the different features of panic. Therefore this study aimed to evaluate the relationship between levels of exercise and the different dimensions of panic symptom severity. Consistent with previous experimental and clinical studies we predicted that higher levels of exercise would be associated to less panic severity related to panic attacks, anticipatory anxiety and disability.

Method: Data from eighty-seven patients with panic disorder as main diagnosis treated at an academic anxiety treatment center were included for the study. The severity of panic attacks, agoraphobic avoidance, anticipatory anxiety, disability and worry about health were used together and individually as main outcome measures. Regular exercise activity/inactivity and gender were used as used independent variables. Additionally, continuous levels of exercise were also used to explore dose-response relationship with levels of panic severity.

Results: After controlling for several potential confounding factors, regression coefficients reveal a significant gender by exercise interaction where exercise activity in men was associated with lower panic severity levels. This association was largely mediated by less disability severity and agoraphobic avoidance severity.

Conclusion: A confirmation of these preliminary findings in a cross-sectional may prove useful for the generation of specific hypotheses to be tested in well-designed clinical trials using aerobic exercise in patients with panic disorder.
\end{abstract}

Key words: physical activity, sports, exercise, panic disorder, panic \& agoraphobia severity scale. 
Introduction

Anxiety disorders constitutes a burden of more than 41,000 million euro to European society (Andlin-Sobocki, Jonsson et al. 2005). Panic disorder and agoraphobia, each affecting about $2 \%$ of the general population, constitute a substantial part of this group of disorders. Panic disorder is a complex condition that often requires multiple forms of treatment such as antipanic pharmacological treatment and psychotherapy (Uhlenhuth, Leon et al. 2006). Problems related to these treatments, such as costs, medication side effects, difficulty with psychotherapy schedules and the related negative stigma often lead to a poor compliance. In this context, alternative forms of therapies such as aerobic exercise are timely. Panic disorder has also been linked to an increased risk of later developing coronary heart disease (GomezCaminero, Blumentals et al. 2005) and is frequently comorbid with asthma (Goodwin and Eaton 2003) and other anxiety disorders as well as major depression (Kessler, Chiu et al. 2005). The benefits of physical aerobic exercise in these conditions have also been clamed (Petruzzello, Landers et al. 1991; Lawlor and Hopker 2001; Ram, Robinson et al. 2005; Stathopoulou, Powers et al. 2006; de Araujo, de Mello et al. 2007; Kokkinos 2008). Physical aerobic exercise has the potential to become a low-cost, stigma-free and readily available alternative antipanic strategy with additional direct health benefits for patients suffering from panic attacks.

Experimental and epidemiological evidence point out to an inverse relationship between levels of physical exercise and panic symptoms (Broocks, Bandelow et al. 1998; Goodwin 2003; Kaiya, Umekage et al. 2005; Esquivel, Diaz-Galvis et al. 2008). To our knowledge only one well designed study has tested the therapeutic effects of exercise in panic disorder (Broocks, Bandelow et al. 1998). This study has shown that an aerobic exercise program has a therapeutic effect comparable to standard treatment with anti-panic medication. An extension of this study published later shows that the beneficial effects of exercise occurred mainly on aspects of panic-related disability and anticipatory anxiety (Bandelow, Broocks et al. 2000). Our group has shown that a single-bout of moderate/hard exercise reduces the susceptibility to panic with inhaled $\mathrm{CO} 2$, a safe and validated model to recreate panic symptoms, in patients with panic disorder (Esquivel, Diaz-Galvis et al. 2008). These latter results are in agreement with several studies that show that acute exercise can reduce the panic symptoms elicited by experimental paradigms in healthy volunteers (Esquivel, Schruers et al. 2002; Strohle, Feller et al. 2005; Smits, Meuret et al. 2008). In a sample presented by Goodwin (2003), psychiatric diagnoses were determined with a comprehensive diagnostic interview where the presence or absence of several common disorders, including panic, were evaluated. The results in this study are consistent with a positive association between levels of physical activity with incidence of panic disorder among other affective 
The relationship between habitual physical activity and symptom severity in panic disorder

disorders. Furthermore, physical activity showed a dose-response relation to the presence an affective disorder. A Japanese survey of 4000 adults revealed the association of dislike of exercise with panic attacks and panic disorder (Kaiya, Umekage et al. 2005). In a sample of panic disorder patients recruited via advertisement, Smits and Zlovensky found a positive relationship between physical inactivity and grater levels of anxiety sensitivity and panic severity (Smits and Zvolensky 2006).

Despite this evidence, the exact nature of the relationship between exercise and panic disorder is still to be characterized. In the case of cross-sectional data, the causal direction on the inverse association between physical activity and panic is surely to be complex and bidirectional. In other words, as much as the engagement in aerobic exercise can have a specific effect on panic symptomatology and even serve as exposure to interceptive sensations; the agoraphobic avoidance, anticipatory anxiety and general dysfunction is likely to make patients exercise less. In many observational studies on the relationship between aerobic exercise and affective disorders, authors are cautious when discussing the direction of causality but frequently recall the experimental studies that show the beneficial effects of aerobic exercise to speculate on this direction (Stephens 1988; Bhui and Fletcher 2000; Goodwin 2003; Schmitz, Kruse et al. 2004; De Moor, Beem et al. 2006; Galper, Trivedi et al. 2006; Onta, Mizoue et al. 2007; Wiles, Haase et al. 2007). On the other hand, the insipient nature of studies that test the effect of aerobic exercise on affective disorders has yielded only a few well-controlled randomized trials from which to draw conclusions. Furthermore, the impossibility of a true placebo condition in exercise trials and experiments poses an inherent constraint that makes evidence, at best, incomplete. With these difficulties, it is tempting to consider the epidemiological, experimental and clinical data together; nevertheless, such an analysis may not be substantiated. For instance most epidemiological studies measure levels of non-specific forms of exercise or physical activity, whereas the bulk of experimental studies specifically show benefits regarding moderate to hard aerobic exercise. Conversely, the specific effects of exercise on different dimensions of psychopathology are not characterized enough in cross sectional samples to aid on the task of making inferences from epidemiological and experimental data.

The preliminary evidence from both single session experiments and clinical trials suggest that the effects of aerobic exercise on affective symptoms may come as the collection of acute improvements and/or the result of a slow accumulation of enduring processes that mediate more pervasive changes [a modified view from that of Salmon (2001)]. If aerobic exercise has a specific and inherent positive effect on panic disorder, many predictions can be advanced. For instance, that the engagement in sports will predict the variability in certain components panic severity. More specifically, with basis on experimental data and that of clinical trials in patients with panic disorder, we hypothesized that the engagement in aerobic 
exercise will be associated with less panic severity and that this will be explained mainly by its association with the severity of panic attacks, disability and anticipatory anxiety than other aspects of panic such as agoraphobic avoidance and worries about health. With evaluation of a large sample of panic disorder patients with quantitative measures of different panic dimensions and habitual physical activity, we further hypothesize a dose-response relationship between these aspects of panic severity and sports physical activity levels. Accordingly, to test these predictions, we use data collected from panic disorder patients on the severity of panic disorder-relevant dimensions and the amount of occupational, sports and leisure physical activity.

\section{Methods}

\section{Subjects}

Eighty-seven patients with panic disorder, with or without agoraphobia in accordance with DSM-IV criteria as main axis I diagnosis were included for the study. Diagnosis required confirmation by at least one experienced clinician and by the use of a structured diagnostic interview such as the MINI (Sheehan, Lecrubier et al. 1998). Only those patients seeking treatment at the Academic Anxiety Center Maastricht were recruited. Participants with a physical condition that would limit the engagement in physical exercise were excluded. The study was approved by the Maastricht Academic Hospital Medical Ethics Committee.

\section{Procedures and instruments}

The acquisition of panic severity and physical activity measures are part of the routine initial evaluation of patients seeking treatment at the anxiety treatment center. Other available file data on regarding potential confounding factors for physical activity and panic severity such as gender, age, education level, number of comorbid axis I diagnosis and current use of antipanic medication data was considered for this study.

The Baecke physical activity questionnaire (Baecke, Burema et al. 1982) is a short questionnaire that has been developed to measure total levels of habitual physical activity, which includes leisure and non-leisure activities over a long period of time. This is a self-administered questionnaire take about 10 minutes to be completed and explores three dimensions of physical activity: 1) occupational physical activity; 2) physical activity during sports; and 3) physical activity during leisure. Possible scores range for 3.00 to 15.00 points. With basis on the response on item 9 "Do you play sport?", patients were classified as exercising or not-exercising for group comparisons. Total scores on the sports dimension was also used as a continuous independent variable. 
The relationship between habitual physical activity and symptom severity in panic disorder

The Panic \& Agoraphobia Scale (PAS) (Bandelow 1995) was specifically constructed for clinical trials. It measures the severity on panic attacks, agoraphobic avoidance, anticipatory anxiety, impairment in social relationships and work and the assumption of somatic disease. Thirteen items across these five dimensions have 5 possible responses from 0 to 4 , producing a possible scores range for 0 to 52 points. A clinician can administer the scale in less than 10 minutes. The total score on this scale will be used as main outcome variable. Sub-scores will also be used to determine the contribution of the potential effects of exercise, as hypothesized, on panic attacks, anticipatory anxiety and disability on the overall panic severity score.

\section{Statistical analysis}

To determine the relationship between exercise and total PAS scores, a regression model was used where the classification of patients by exercise activity or inactivity was used as independent factors and total PAS scores as dependent variable. Because several crosssectional studies report on significant gender differences in the association between physical activity and affective symptoms (Stephens 1988; Bhui and Fletcher 2000; Wendel-Vos, Schuit et al. 2004; Onta, Mizoue et al. 2007; Wiles, Haase et al. 2007), gender and the gender by exercise interaction were also included in the regression model as predictors. Potential confounding factors for both panic severity and exercise such as the number of currently used antipanic medication, number of comorbid axis-1 conditions, age and level of education were also included in the model.

The same procedure was performed using all dimensions of the PAS scale (panic attacks, agoraphobic avoidance, anticipatory anxiety, disability and worries about heath) individually as the dependent variable. The regression coefficients produced from each model were compared. Finally, to investigate potential dose-response associations between physical exercise and potential relevant PAS dimensions, the (centered) Baecke sports physical activity index score will be use as a continuous variable in place of the dichotomous classification of exercise status. Results with $p$ values less than 0.05 were interpreted as significant.

\section{Results}

Eighty-four subjects were included in the analyses. Three subjects were excluded because of incomplete data. The overall simple comparison on the different measures when groups ware classified in not-exercising and exercising is given in Table 1. Total panic severity symptoms did not differ between patients that exercised regularly and those that did not. However when patients are further classified by gender, male patients that exercise have a lower total panic severity scores when compared to those that do not. With regard to potential confounding 
Chapter 7

factors, there were no group differences in age, proportion of females and males, number of axis I diagnoses and educational level; although, those that exercised regularly tended to used less anti-panic medication. All subjects classified as exercising preformed aerobic forms of exercise.

Table 1. Comparison between the groups classified as not-exercising and exercising

\begin{tabular}{lll}
\hline & Not-exercising $(N=46)$ & Exercising $(N=38)$ \\
\hline Age & $38.03(11.8)$ & $38.86(10.3)$ \\
Female/male ratio & $32 / 14$ & $23 / 15$ \\
Total PAS score & $23.46(10.3)$ & $21.13(10.4)$ \\
$\quad$ Female & $22.78(10.1)$ & $24.35(9.5)$ \\
$\quad$ Male & $25.00(8.6)$ & $16.2(10.0)$ \\
Number of Medications & $1.00(0.7)$ & $0.68(0.8)$ \\
Number of axis I diagnoses & $2.07(1.0)$ & $2.21(1.0)$ \\
Educational level (1-3) & $2.26(0.5)$ & $2.37(0.6)$ \\
\hline PAS: Panic \& Agoraphobia scale. Mean scores (+/- SD). P: uncorrected \\
probability of no difference between groups (unpaired t-test or $\chi^{2}$, two-talled)
\end{tabular}

Table 2. Association between exercise and panic severity

\begin{tabular}{lcccccc}
\hline & $B$ & SE $B$ & $\beta$ & $P=$ & $95 \%$ CI for $B$ \\
\hline Step 1 Constant & 24.303 & 1.684 & & & & \\
Exercise & -2.783 & 2.378 & -.129 & .245 & -6.592 & 2.445 \\
Gender & -2.073 & 2.271 & -.100 & .364 & -7.513 & 1.948 \\
\hline Step 2 Constant & 22.781 & 1.780 & & & & \\
Exercise & 2.219 & 3.227 & .103 & .494 & -3.911 & 7.044 \\
Gender & 1.567 & 2.753 & .076 & .571 & -4.202 & 8.640 \\
Exercise x Gender & -10.367 & 4.645 & -.386 & .028 & -19.611 & -1.122 \\
\hline Step 3 Constant & \multicolumn{7}{c}{8.572} \\
Exercise & 2.695 & 3.266 & .125 & .412 & 7.044 & 6.905 \\
Gender & 1.386 & 2.771 & .067 & .618 & 8.640 & 9.200 \\
Exercise x Gender & -9.531 & 4.737 & -.355 & .048 & -1.122 & -.096 \\
Age & -.073 & .111 & -.077 & .513 & 7.044 & .148 \\
\# Diagnoses & 1.332 & 1.124 & .129 & .240 & 8.640 & 3.570 \\
\# Anti-panic Medications & .377 & 1.589 & .028 & .813 & -1.122 & 3.541 \\
Level of education & -3.023 & 2.316 & -.157 & .196 & 7.044 & 1.590 \\
\hline
\end{tabular}

In step 1, to observe the main effects, exercise condition and gender are entered in the model. In step 2, to observe the interaction effect, exercise condition, gender and the interaction between these are entered in the model. In step 3, age, number of comorbid axis I conditions, number of currently used anti-panic medications and education level were also entered to control for their potential confounding effect on the association between exercise and panic severity 
The relationship between habitual physical activity and symptom severity in panic disorder

Table 2 displays the coefficients of the regression model to analyze the association between exercise in patients with panic disorder and panic severity. Main effects for gender and exercise was not significant. When exercise, gender and the interaction exercise by gender were included in the model as a second step; the interaction could significantly predict the total scores on the PAS. As noted from Table 1, this interaction effect is due to the difference in mean total PAS scores between men that exercise and those that do not. This effect is further illustrated in Figure 1. This means that in men, exercising was associated with a decrease in panic severity of .386 the total score. When controlling for potential confounding factors such as age, number of currently taken anti-panic medication, current axis 1 comorbidity and educational level; exercising in men was still significantly associated with a decrease in panic severity of .355 the total score.

To compare the strength of the associations between the exercise by gender interaction with each panic dimension of the PAS scale (panic attacks, agora-phobic avoidance, anticipatory anxiety, disability and worries about health), these were separately used as the dependent variable in the regression model. Table 3 shows the regression coefficients for the exercise by gender interaction on each dimension, before and after controlling for potential confounding factors. Only the association between the interaction and disability displayed significant coefficients, both before and after controlling for potential confounding factors; exercising in men was related to a reduction in the disability sub-score of .447 and .395 , respectively. In a similar way, the association between the interaction and agoraphobic avoidance displayed a trend towards significant coefficients. Notably, although not reported on Table 3 , the number of currently used anti-panic medication significantly predicted the severity of panic attacks even when controlling for all other predictors $[\beta=-.270$ (standardized), $P=.031]$.

To further investigate the relationship between the exercise by gender interaction and the PAS disability and agoraphobic avoidance sub-scores; the Baecke sports physical activity index was used as a continuous variable of exercise. In a first step, gender, sports physical activity levels and the interaction between these were entered in a regression model as predictor of each PAS subscale (See Table 4). In a second step, potential confounding factors were also entered. The interaction between gender and sports physical activity levels showed a trend towards a significant association with disability sub-scores of the PAS. This trend was no longer present after controlling for confounders. On the other hand, the relationship between the interaction and agoraphobic avoidance was not significant in either steps of the model. 
Chapter 7

Figure 1. The association between physical exercise status and gender with to-tal PAS scores

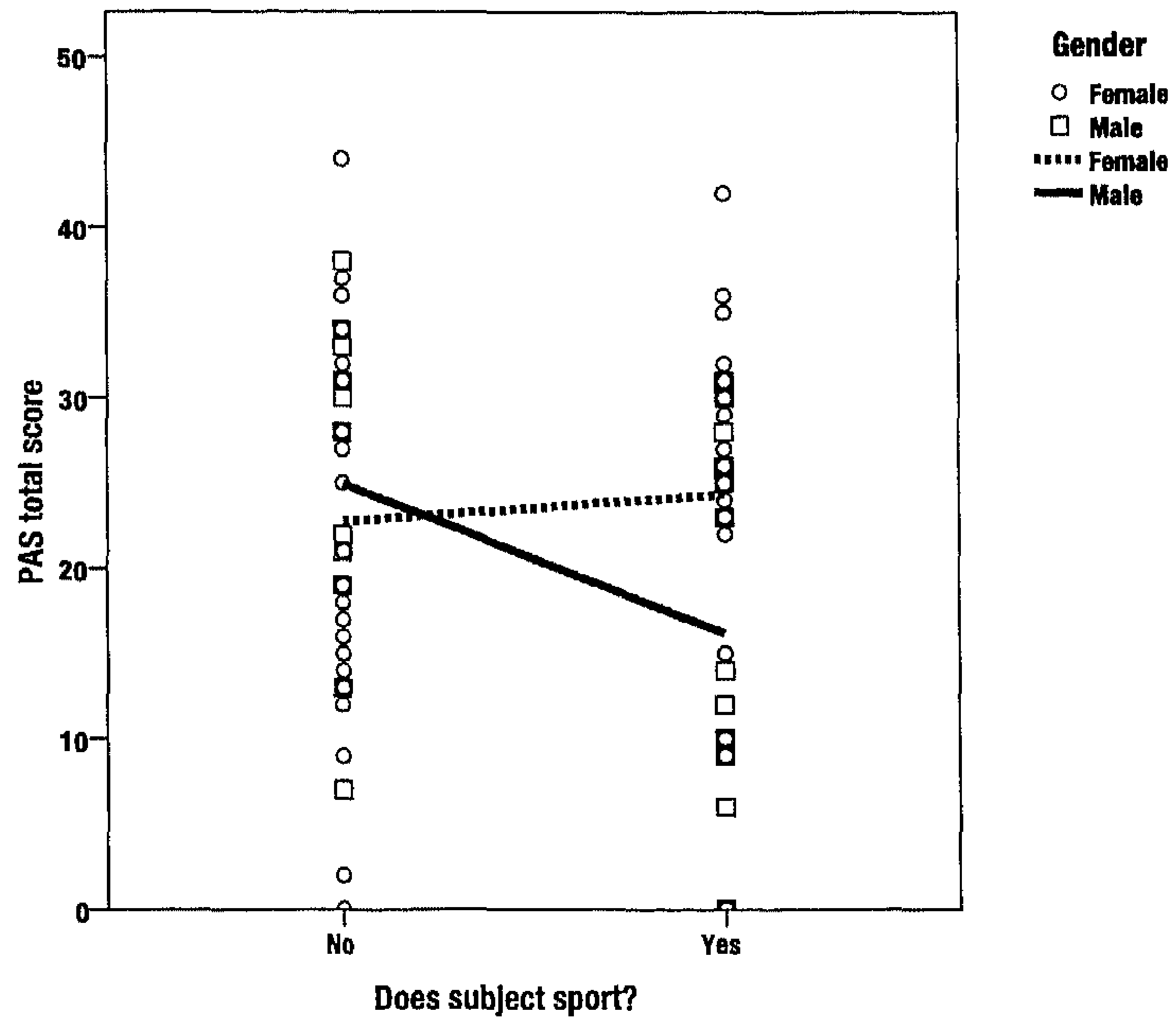

Scatter plot of relationship between total PAS scores and physical exercise status. Data points for each gender are plotted using different symbols. Regression lines represent the relationship between total PAS scores and physical exercise status for each gender

Table 3. Association between exercise and panic severity dimensions

\begin{tabular}{llllllll}
\hline & Dependent variable & $\boldsymbol{B}$ & SE $\boldsymbol{B}$ & $\boldsymbol{\beta}$ & $\boldsymbol{P}=$ & \multicolumn{2}{c}{$\mathbf{9 5 \%}$ Cl for $\boldsymbol{B}$} \\
\hline Step 1 & Panic attacks & -1.248 & 1.623 & -.141 & .445 & -4.482 & 1.986 \\
Exercise $x$ & Agoraphobic avoidance & -3.994 & 2.092 & -.341 & .060 & -8.161 & .174 \\
Gender & Anticipatory anxiety & -.501 & 1.290 & -.072 & .699 & -3.072 & 2.069 \\
& Disability & -4.307 & 1.7 & -.447 & .013 & -7.693 & -.920 \\
& Worries about Health & .747 & 1.0 & .137 & .457 & -1.245 & 2.738 \\
\hline Step 2 & Panic attacks & -1.164 & 1.621 & -.185 & .315 & -4.872 & 1.593 \\
Exercise x & Agoraphobic avoidance & -3.821 & 2.169 & -.327 & .082 & -8.147 & .504 \\
Gender & Anticipatory anxiety & -.382 & 1.336 & -.055 & .776 & -3.046 & 2.282 \\
& Disability & -3.809 & 1.7 & -.395 & .028 & -7.199 & -.418 \\
& Worries about Health & .971 & 1.019 & .178 & .334 & -1.061 & 3.003 \\
\hline
\end{tabular}

In step 1, to observe the interaction effect, exercise condition, gender and the interaction between these are entered in the model. In step 2, age, number of comorbld axis I conditions, number of currently used anti-panic medications and education level were also entered to control for their potential confounding effect on the association between exercise and panic severity 
The relationship between habitual physical activity and symptom severity in panic disorder

Table 4. Dose-response associations between sports physical activity levels and the subscales of agoraphobic avoidance and disability

\begin{tabular}{|c|c|c|c|c|c|c|c|}
\hline \multirow{3}{*}{$\begin{array}{l}\text { Step } 1 \\
\text { Sports physical activity } \\
\text { level } l^{\star} \times \text { Gender }\end{array}$} & \multirow{3}{*}{$\begin{array}{l}\begin{array}{l}\text { Dependent } \\
\text { variable }\end{array} \\
\text { Disability } \\
\text { Agoraphobic } \\
\text { avoidance }\end{array}$} & \multirow{3}{*}{$\begin{array}{c}B \\
-1.926 \\
-1.471\end{array}$} & \multirow{3}{*}{$\begin{array}{c}\text { SE B } \\
1.032 \\
1.258\end{array}$} & \multirow{3}{*}{$\begin{array}{c}\beta \\
-.271 \\
-.170 \\
\end{array}$} & \multirow{3}{*}{$\begin{array}{c}P= \\
.066 \\
.246\end{array}$} & \multicolumn{2}{|c|}{$95 \% \mathrm{Cl}$ for B } \\
\hline & & & & & & -3.982 & .131 \\
\hline & & & & & & -3.978 & 1.036 \\
\hline Step 2 & Disability & -1.537 & 1.054 & -.216 & .149 & -3.368 & .564 \\
\hline $\begin{array}{l}\text { Sports physical activity } \\
\text { level }{ }^{*} \times \text { Gender }\end{array}$ & $\begin{array}{l}\text { Agoraphobic } \\
\text { avoidance }\end{array}$ & -1.340 & 1.331 & -.155 & .317 & -3.993 & 1.313 \\
\hline
\end{tabular}

In step 1 , to observe the interaction effect, sports physical activity level, gender and the interaction between these are entered in the model. In step 2, age, number of comorbid axis I conditions, number of currently used anti-panic medications and education level were also entered to control for their potential confounding effect on the association between exercise and panic severity. ${ }^{*}$ These scores were centered

\section{Discussion}

In our sample of patients with panic disorder, the lack of regular physical exercise was associated with larger total PAS scores only in men. Although a substantial number of crosssectional studies in to the relationship between exercise and affective disorders do not find gender differences, several do report a larger or exclusive association in men (Bhui and Fletcher 2000; Wendel-Vos, Schuit et al. 2004; Ohta, Mizoue et al. 2007; Wiles, Haase et al. 2007). Smits and Zvolensky (2006) also inquired panic severity symptoms and physical activity behavior in a sample of panic disorder patients and found no apparent gender differences on the relationship between physical inactivity and increase panic severity. If we compare our sample with that reported by Smits and Zvolenski, the evident differences are the average patient age ( $t=-6.32, P<.05$, two-tailed) and method of subject recruitment. These differences may be relevant in the form of illness duration and accumulated comorbidity, and the inherent characteristics of individuals who seek treatment and those that respond to advertisements to participate in research. For instance, many individuals whom may have benefited substantially from exercise would not seek treatment at a specialized clinic, but may volunteer for research.

Further in our analyses we had planned to look at the potential different strengths of the predictors in models where each of the dimensions that make up the total PAS score are used as a dependent variable. We had hypothesized, with basis on experimental data, that scores on panic attacks, anticipatory anxiety and disability would show the most robust relationships with exercise behavior. However, regression coefficients were only significant for an association between the gender by exercise interaction and PAS disability scores. Notably, a trend for a significant association with agoraphobic avoidance was also present. These 
finding are compatible, at least in part, with clinical studies on the effects of exercise therapy on disability and anticipatory anxiety severity measures in panic disorder patients (Bandelow, Broocks et al. 2000). Notwithstanding, it must be noted that is plausible for both, exercise to reduce disability, as it is for disability to reduce the amount of exercise to be practiced by an individual. Furthermore, less disability could be interpreted as a result from lower anticipatory anxiety or agoraphobic avoidance, as found in the mentioned clinical study and our sample respectively. On the other hand, the stronger association between exercise behavior in men and disability and the fact that disability can take an independent course from agoraphobia and other key panic disorder symptoms (Katschnig. Amering et al. 1996) suggests some specificity for exercise behavior as a predictor of disability.

We did not find that exercise was related to lower scores on panic attacks or anticipatory anxiety. The mentioned experimental and clinical studies suggested that for acute anti-panic effects and effects on anticipatory anxiety and disability to take place, moderate to hard levels of physical activity are necessary. In our sample of patients, less than a half of those that performed regular exercise did so in a way roughly equivalent to moderate/hard exercise. On the other hand, the effects of exercise on physiological and psychological adaptation to stress seem to require less intense levels of aerobic exercise (Salmon 2001) and little physical efforts are required for exposure to agoraphobic triggers. Low levels of exercise may better relate to lower scores in agoraphobic avoidance and disability. Also relevant, was a significant association between the number of currently used anti-panic medication and PAS panic attack scores. Although the number of anti-panic medications was a rather crude index simply counting the number of benzodiazepine and antidepressant medications regardless of type and dosage, each medication taken was associated with a PAS panic attacks score point reduction of .27. Anti-panic pharmacologic therapy, taken by a majority of our sample of patients, could have obscured a modest relationship between exercise and panic attack severity.

Many limitations in the present study must be taken into account when drawing conclusions. The relatively small sample and crude measures to control for confounding factors size renders the results as preliminary. To this time point, the results from a crosssectional sample taken from a single academic anxiety treatment center do not allow to generalize any findings beyond this group. In our sample, the association between the levels of sports physical activity by gender interaction and PAS disability scores was only marginally significant when uncontrolled for confounders. The inclusion of a larger sample size and samples from other treatment groups could overcome these limitations and confirm the present associations.

Our preliminary results indicate that the lack of regular physical exercise was associated with more panic symptom severity in men. This association can be largely 
The relationship between habitual physical activity and symptom severity in panic disorder

explained by relationship between the gender by exercise interaction and PAS disability scores. These finding are compatible, at least in part, with clinical studies on the effects of exercise therapy on disability and anticipatory anxiety severity measures in panic disorder patients. A confirmation of these findings in a cross-sectional sample would not allow any conclusions regarding causality, but may prove useful for the generation of specific hypotheses to be tested in well-designed clinical trials using aerobic exercise in patients with panic disorder. The Panic \& Agoraphobia scale can be used to determine how different types of exercise conditions (e.g. isolation, group, outdoors) and intensities can modify discrete dimensions of panic symptom severity. The benefits of aerobic exercise in panic are still to be further demonstrated, characterized and used for the development of neurobiological models in to the mechanisms of acute and sustained anti-panic treatment. 


\section{References}

Andlin-Sobocki, P., B. Jonsson, et al. (2005). "Cost of disorders of the brain in Europe." Eur J Neurol 12 Suppl 1: 1-27

Baecke, J. A., J. Burema, et al. (1982). "A short questionnaire for the measurement of habitual physical activity in epidemiological studies." Am J Clin Nutr 36(5): 936-42.

Bandelow, B. (1995). "Assessing the efficacy of treatments for panic disorder and agoraphobia. II. The Panic and Agoraphobia Scale." Int Clin Psychopharmacol 10(2): 73-81.

Bandelow, B., A. Broocks, et al. (2000). "The use of the Panic and Agoraphobia Scale (P \& A) in a controlled clinical trial." Pharmacopsychiatry 33(5): 174-81.

Bhui, $K$. and A. Fletcher (2000). "Common mood and anxiety states: gender differences in the protective effect of physical activity." Soc Psychiatry Psychiatr Epidemiol 35(1): 28-35.

Broocks, A., B. Bandelow, et al. (1998). "Comparison of aerobic exercise, clomipramine, and placebo in the treatment of panic disorder." Am J Psychiatry 155(5): 603-9.

de Araujo, S. R., M. T. de Mello, et al. (2007). "[Anxiety disorders and physical exercise]." Rev Bras Psiquiatr 29(2): 164-71.

De Moor, M. H., A. L. Beem, et al. (2006). "Regular exercise, anxiety, depression and personality: a population-based study." Prev Med 42(4): 273-9.

Esquivel, G., J. Diaz-Galvis, et al. (2008). "Acute exercise reduces the effects of a $35 \% \mathrm{CO}(2)$ challenge in patients with panic disorder." JAffect Disord 107(1-3): 217-20.

Esquivel, G., K. Schruers, et al. (2002). "The effects of acute exercise and high lactate levels on $35 \% \mathrm{CO} 2$ challenge in healthy volunteers." Acta Psychiatr Scand 106(5): 394-7.

Galper, D. I., M. H. Trivedi, et al. (2006). "Inverse association between physical inactivity and mental health in men and women." Med Sci Sports Exerc 38(1): 173-8.

Gomez-Caminero, A., W. A. Blumentals, et al. (2005). "Does panic disorder increase the risk of coronary heart disease? A cohort study of a national managed care database." Psychosom Med 67(5): 688-91.

Goodwin, R. D. (2003). "Association between physical activity and mental disorders among adults in the United States." Prev Med 36(6): 698-703.

Goodwin, R. D. and W. W. Eaton (2003). "Asthma and the risk of panic attacks among adults in the community." Psychol Med 33(5): 879-85.

Kaiya, H., T. Umekage, et al. (2005). "Factors associated with the development of panic attack and panic disorder: survey in the Japanese population." Psychiatry Clin Neurosci 59(2): 177-82.

Katschnig, H., M. Amering, et al. (1996). "Predictors of quality of life in a long-term followup study in panic disorder patients after a clinical drug trial." Psychopharmacol Bull 32(1): 149-55.

Kessler, R. C., W. T. Chiu, et al. (2005). "Prevalence, severity, and comorbidity of 12-month DSM-IV disorders in the National Comorbidity Survey Replication." Arch Gen Psychiatry 62(6): 617-27.

Kokkinos, P. (2008). "Physical activity and cardiovascular disease prevention: current recommendations." Angiologv 59 (2 Suppl): 26S-9S.

Lawlor, D. A. and S. W. Hopker (2001). "The effectiveness of exercise as an intervention in the management of depression: systematic review and meta-regression analysis of randomised controlled trials." Bmi 322(7289): 763-7.

Ohta, M., T. Mizoue, et al. (2007). "Effect of the physical activities in leisure time and commuting to work on mental health." J Occup Health 49(1): 46-52.

Petruzzello, S. J., D. M. Landers, et al. (1991). "A meta-analysis on the anxiety-reducing effects of acute and chronic exercise. Outcomes and mechanisms." Sports Med 11(3): 143-82.

Ram, F. S., S. M. Robinson, et al. (2005). "Physical training for asthma." Cochrane Database Syst Rev(4): CD001116.

Salmon, P. (2001). "Effects of physical exercise on anxiety, depression, and sensitivity to stress: a unifying theory." Clin Psychol Rev 21(1): 33-61.

Schmitz, N., J. Kruse, et al. (2004). "The association between physical exercises and health-related quality of life in subjects with mental disorders: results from a cross-sectional survey." Prev Med 39(6): 1200-7. 
The relationship between habitual physical activity and symptom severity in panic disorder

Sheehan, D. V., Y. Lecrubier, et al. (1998). "The Mini-International Neuropsychiatric Interview (M.I.N.I.): the development and validation of a structured diagnostic psychiatric interview for DSM-IV and ICD10." J Clin Psychiatry 59 Suppl 20: 22-33;quiz 34-57.

Smits, J. A., A. E. Meuret, et al. (2008). "The effects of acute exercise on $\mathrm{CO}(2)$ challenge reactivity." $\mathrm{J}$ Psvchiatr Res.

Smits, J. A. and M. J. Zvolensky (2006). "Emotional vulnerability as a function of physical activity among individuals with panic disorder." Depress Anxiety 23(2): 102-6.

Stathopoulou, G., M. B. Powers, et al. (2006). "Exercise Interventions for Mental Health: A Quantitative and Qualitative Review." Clinical Psychology: Science and Practice 13(2): 179-193.

Stephens, T. (1988). "Physical activity and mental health in the United States and Canada: evidence from four population surveys." Prev Med 17(1): 35-47.

Strohle, A., C. Feller, et al. (2005). "The acute antipanic activity of aerobic exercise." Am J Psychiatry 162(12): 2376-8.

Uhlenhuth, E. H., A. C. Leon, et al. (2006). "Psychopathology of panic attacks in panic disorder." $\underline{J \text { Affect }}$ Disord 92(1): 55-62.

Wendel-Vos, G. C., A. J. Schuit, et al. (2004). "Leisure time physical activity and health-related quality of life: cross-sectional and longitudinal associations." Qual Life Res 13(3): 667-77.

Wiles, N. J., A. M. Haase, et al. (2007). "Physical activity and common mental disorder: results from the Caerphilly study." Am J Epidemiol 165(8): 946-54. 


\section{Chapter 8:}

\section{Acids in the brain: A factor in panic?}

Esquivel $G^{1}$, Schruers $K^{1}$, Maddock $R J^{2}$, Colasanti $A$ and Griez $E^{1}$

'School for Mental Health and Neurosciences and Academic Anxiety Center, Maastricht University, PO Box 616, 6200 MD, Maastricht, The Netherlands

${ }^{2}$ Department of Psychiatry and Imaging Research Center, University of California Davis Medical Center, Sacramento, CA, USA

Accepted for publication as: Esquivel, G., K. Schruers, R. J. Maddock, A. Colasanti and E. Griez (2009). "Acids in the brain. A factor in panic?" J Psychopharmacol (Accepted) 
Acids in the brain: A factor in panic?

\begin{abstract}
Several methods to experimentally induce panic cause profound acid-base disturbances. Evidence suggests that $\mathrm{CO} 2$ inhalations, lactate infusions and, to a certain extent, voluntary hyperventilation can conceivably lead to a common scenario of brain acidosis in the face of disparate intravascular $\mathrm{pH}$ alterations. The importance of this event is reflected in data that support a model in which experimental panic attacks, as proxy to those occurring spontaneously, constitute a response to acute brain acidosis. Given that central $\mathrm{CO} 2 / \mathrm{H}_{+}$ chemoreception is an important drive for ventilation, and many chemosensitive neurons are related to respiration and arousal, this model can explain much of the connection between panic and respiration. We propose that the shared characteristics of $\mathrm{CO} 2 / \mathrm{H}+$ sensing neurons overlap to a point where threatening disturbances in brain $\mathrm{pH}$ homeostasis, such as those produced by $\mathrm{CO} 2$ inhalations, elicit a primal emotion that can range from breathlessness to panic.
\end{abstract}

Keywords: panic, anxiety, $\mathrm{pH}$, acid-base disturbance, carbon dioxide, lactate, voluntary hyperventilation. 
Chapter 8

Introduction

The underlying mechanisms by which various techniques can experimentally induce panic attacks remain elusive. A pragmatic approach to searching for clues is to determine a common denominator among the different methods that are claimed to provoke panic. A prerequisite for such models is their ability to specifically and reliably induce panic attacks that are susceptible to standard anti-panic treatment. In other words, they should be reasonably valid models of panic. In this context, one observation frequently made is that several of these extensively used panic-inducing methods, such as carbon dioxide inhalations and sodium lactate infusions, elicit important acid-base disturbances. At first glance, the changes produced in the body are disparate, as blood-gas analysis reveals a respiratory acidosis and a metabolic alkalosis, with $\mathrm{CO} 2$ inhalations and lactate infusions, respectively. In previous publications attempts to reconcile these apparently disparate $\mathrm{pH}$ responses were limited by the available data (Carr and Sheehan 1984; Zandbergen, Pols et al. 1989; Griez and Schruers 1998; Schruers, Klaassen et al. 2000). In this paper, we will review the acid-base physiological effects of several provocation challenges. We will include recently generated data as a framework within which panic is explained as a defensive response related to acute brain acidosis.

Respiratory symptoms are among the most common complaints in panic disorder patients (Schruers, van de Mortel et al. 2004) and are often present in spontaneous panic attacks (Briggs, Stretch et al. 1993). These findings, and the fact that inhaled CO2 and infused sodium lactate act as respiratory stimulants, have encouraged much research into the relationship between panic disorder and respiratory physiology as a potential clue to understanding panic. Although the exact nature of this relationship has yet to be determined, a compelling amount of research supports a respiratory abnormality in panic disorder (Stein, Millar et al. 1995; Abelson, Weg et al. 2001; Yeragani, Radhakrishna et al. 2002; Caldirola, Bellodi et al. 2004). Maddock (2001) has suggested that these abnormal respiratory findings may reflect an underlying metabolic disturbance characterized by exaggerated brain lactate responses. While, in our earlier theoretical work (Griez and Schruers 1998), we posed the question "Why should an accumulation of these substances (lactate and CO2) cause panic in susceptible individuals?", we now propose that the administration of $\mathrm{CO} 2$ and lactate elicit spontaneous panic attacks as a result of the activation of networks that monitor brain $\mathrm{pH}$.

In addition to the complex clinical picture of panic disorder (i.e., situational and spontaneous panic attacks, anticipatory anxiety, phobic avoidance), a great deal of heterogeneity can be found in the symptomatic expression of spontaneous panic attacks. Cluster analyses of symptoms reported by patients with panic disorder during spontaneous attacks have identified several panic attack subtypes. Those with predominantly respiratory 
Acids in the brain: A factor in panic?

symptoms respond best to imipramine (Briggs, Stretch et al. 1993) and are mostly sensitive to CO2 challenges (Abrams, Rassovsky et al. 2006; Nardi, Valenca et al. 2006). In healthy volunteers subjected to $\mathrm{CO} 2$ challenges and in whom a distinct respiratory cluster is found, respiratory symptoms are the best predictors of a subjective, fearful response (Colasanti, Salamon et al. 2008). The presence of this subtype of panic attack is consistent with a defensive response closely related to respiration and $\mathrm{pH}$ homeostasis. Here, our presentation will be restricted to the relevant experimental data in order to establish a link between brain acid-base status and experimental panicogenic maneuvers.

The acid-base physiology of experimental panicogenic challenges

Carbon dioxide

An end-product of carbohydrate metabolism, $\mathrm{CO} 2$ is constantly produced in the body, readily processed by the bicarbonate system, and transported from tissues to the lungs, where it is finally excreted. Increased levels of carbon dioxide in the body elicit powerful ventilatory stimulation mediated by peripheral and central $\mathrm{CO} 2 / \mathrm{H}+$ chemoreceptors. In the presence of carbonic anhydrase, $\mathrm{CO} 2$ interacts with water to form carbonic acid ( $\mathrm{H} 2 \mathrm{CO} 3)$, which rapidly dissociates into $\mathrm{H}+$ and bicarbonate ( $\mathrm{HCO}-$ ). As the blood-brain barrier is very permeable to $\mathrm{CO} 2$, changes in end-tidal pCO2 (partial pressure of CO2) are inversely related to brain $\mathrm{pH}$ (Jensen, Thomsen et al. 1988; Cadoux-Hudson, Rajagopalan et al. 1990; Martoft, StodkildeJorgensen et al. 2003). Therefore, the result of respiratory acidosis caused by inhaling a high concentration of $\mathrm{CO} 2$ is a decrease in $\mathrm{pH}$ (an increase in $\mathrm{H}+$ ) in most body fluid compartments and across the blood-brain barrier.

The experimental panicogenic properties of $\mathrm{CO} 2$ have been described since 1951 (Cohen and White 1951), but it was not until the mid-1980s that Griez and Van den Hout (1984) at Maastricht University used inhalations with 35\% CO2, which was considered "anxiolytic" in previous reports. With this inhalation, panic disorder patients experienced intense physical and cognitive symptoms that closely resembled those of spontaneous panic attacks. At the same time, Gorman (1984) and coworkers at Columbia University in New York used continuous inhalations of $5 \% \mathrm{CO} 2$ as a means of controlling hypocapnia during voluntary hyperventilation and unexpectedly found $\mathrm{CO} 2$ to be more panicogenic. Since then, the methods used to administer $\mathrm{CO} 2$ as a panic provocation challenge have become widely diversified (Rassovsky and Kushner 2003).

Much experimental data suggest that the intensity of panic symptoms elicited by $\mathrm{CO} 2$ is contingent upon the dose administered. A comparison of panic rates in studies using a double inhalation versus a single inhalation or the continuous inhalations of $7 \%$ versus $5 \%$ $\mathrm{CO} 2$ shows that a larger dose invariably results in a higher panic rate in panic disorder patients 
and in healthy controls (Gorman, Papp et al. 1994; Rassovsky and Kushner 2003; Nardi, Valenca et al. 2006). In a study specifically designed to address this issue, healthy subjects who were randomly subjected to a double inhalation of different dosages of CO2 reported an intensity of panic symptoms contingent on the amount of $\mathrm{CO} 2$ administered (Griez, Colasanti et al. 2007). A noteworthy finding in this study is that when healthy subjects inhaled high concentrations of $\mathrm{CO} 2$, they reported both somatic and cognitive symptoms compatible with current nosological definitions of a panic attack.

Are brain levels of $\mathrm{CO} 2$, as opposed to peripheral levels, relevant in the panic response? Two groups of researchers (Mathew, Wilson et al. 1989; Gorman, Papp et al. 1993) tested the subjective effects of acetazolamide, a carbonic anhydrase inhibitor, in panic disorder patients. This drug was believed to increase brain pCO2 without stimulating peripheral chemoreceptors. Since acute acetazolamide administration in panic disorder patients did not provoke panic, it was initially suggested that increases in $\mathrm{CO} 2$ in the brain were not involved in experimental panic. However, since, in these experimental conditions, the maneuver did not stimulate respiration-a well-established response to increased brain $\mathrm{CO} 2$ and acetazolamide (Swenson and Hughes 1993) - these results are inconclusive.

\section{Sodium lactate}

Lactate, the dissociated anionic form of lactic acid in biological fluids, is a ubiquitous substance that is constantly produced as a result of mammalian metabolism. It is actively recycled and may play a major role in energetic homeostasis in both physiological and extreme conditions. Lactate, glycolytically converted from glucose in astrocytes, may be an important energy source for neurons (Tsacopoulos and Magistretti 1996). Unlike CO2, the blood-brain barrier permeability to lactate is limited by specific transporters. In certain circumstances, such as physical exertion, net lactate transport into the brain can increase (Dalsgaard, Quistorff et al. 2004).

The standardized use of sodium lactate infusions as a panicogenic maneuver was established by Pitts and McClure (1967) and is perhaps the most extensively studied experimental model of panic. Their idea stemmed from the previous work of Cohen and White (1951), in which patients with neurocirculatory asthenia (a diagnostic entity overlapping with panic disorder) showed abnormally high blood lactate levels and a low oxygen uptake in response to physical exertion. Sodium lactate is usually administered in its racemic formequal amounts of $D$ - and L-lactate - in a 10-mL/kg hyperosmolar concentration of $0.5 \mathrm{~mol} / \mathrm{L}$ for 20 minutes. The effects of pure D- or L-sodium lactate infusions on panic patients, although not clear-cut, suggest that they produce results similar to those with racemic mixtures (Gorman, Goetz et al. 1990; Klein 1993). The infusion produces a metabolic alkalosis 
Acids in the brain: A factor in panic?

largely restricted to the intravascular space. Here, the key factors leading to a higher $\mathrm{pH}, \mathrm{H}+$ and HCO3- cannot readily cross the blood-brain barrier.

In a sodium lactate infusion, the physiological process that leads to a metabolic alkalosis apparently occurs primarily as a result of the simultaneous removal of lactate and $\mathrm{H}+$ by the liver (and, to some extent, by the heart and brain). The removal of $\mathrm{H+}$, in the presence of $\mathrm{CO} 2$ and carbonic anhydrase, results in a net increase in blood bicarbonate. In a study designed to test the involvement of bicarbonate and alkalosis in lactate-induced panic, patients with panic disorder received both a standard racemic sodium lactate infusion and a 20 -minute infusion of $2.5 \mathrm{~mL} / \mathrm{kg}$ of $8.4 \%$ sodium bicarbonate (Gorman, Battista et al. 1989). Both infusions were panicogenic and acted as a respiratory stimulant (paradoxical in light of a metabolic alkalosis, as measured in blood-gas analysis) in subjects responding to the maneuvers. Notably, whereas a lactate infusion stimulated respiration in all subjects, the infusion with bicarbonate did so only in the responders.

Does bicarbonate have a role in the panicogenic and respiratory effects of lactate? Other work shows that a sodium bicarbonate infusion can paradoxically cause brain acidosis. Phosphorous-magnetic resonance spectroscopy (MRS) studies in human volunteers (Nakashima, Yamashita et al. 1996) and in animals (Kucera, Shapiro et al. 1989; Shapiro, Whalen et al. 1989) have demonstrated lowering of intracellular $\mathrm{pH}$ (pHi) in the brain during an infusion with sodium bicarbonate despite an intravascular metabolic alkalosis. Other animal studies conducted to investigate cerebral spinal fluid acid-base shifts during an intravenous

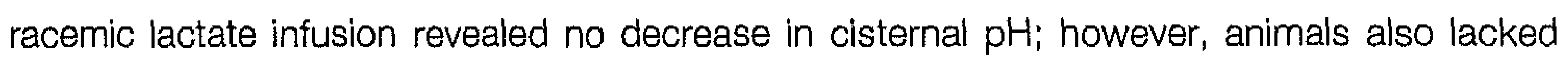
any sign of ventilatory stimulation or were mechanically ventilated (Dager, Rainey et al. 1990; Coplan, Sharma et al. 1992). To this point, we can only speculate that hyperventilation and the consequent decrease in $\mathrm{pCO} 2$ during a lactate infusion is a response to an initial brain acidification.

An additional, or alternative, mechanism by which a lactate infusion can further disturb brain $\mathrm{pH}$ is the well-documented increased (endogenous) brain lactate response displayed by panic patients after this challenge (Dager, Marro et al. 1994; Dager, Richards et al. 1997; Dager, Friedman et al. 1999). This endogenous lactate increase-unlike sodium lactate infused peripherally - can act as a strong acid anion and lead to a net decrease in $\mathrm{pH}$. This is consistent with a finding in a study performed on monkeys with in situ biosensor measurements of arterial blood, cerebrospinal fluid, and brain tissue, where a lactate infusion was related to a trend for parenchymal $\mathrm{pO} 2$ and $\mathrm{pH}$ to decrease (Dager, Yim et al. 1995). These findings are revealing as they suggest a shift in metabolism leading to an accumulation of lactate and $\mathrm{CO} 2$. In fact, pCO2 rose in brain tissue while arterial levels decreased. As mentioned, the lactate produced in astrocytes during normal brain activation is co-transported along with $\mathrm{H}+$ into the extracellular space (Dienel and Hertz 2001). This is said to be 
responsible for the extracellular space acidosis associated with neuronal activation (Scheller, Kolb et al. 1992).

\section{Voluntary hyperventilation}

The fact that fast breathing can induce symptoms that overlap with panic (e.g., dizziness, paresthesia), together with the observation that a decrease in pCO2 during a sodium lactate infusion precedes a panic response, prompted the use of voluntary hyperventilation as a pathophysiological model for panic (Lum 1987). In contrast to $\mathrm{CO} 2$ and lactate, voluntary hyperventilation is a weak and inconsistent method for inducing panic (Griez, Zandbergen et al. 1988; Zandbergen, Lousberg et al. 1990; Zandbergen, Pols et al. 1991; Papp, Martinez et al. 1997; Goetz, Klein et al. 2001). Nevertheless, the practical and non-invasive features of voluntary hyperventilation have yielded a compelling amount of research (Maddock 2001; Nardi, Lopes et al. 2004; Nardi, Valenca et al. 2004). Most techniques involve either visual or auditory stimuli to indicate the frequency of 30 breathing cycles per minute for 4 to 15 minutes (Papp, Martinez et al. 1997; Nardi, Valenca et al. 2001) or feedback for the subject to breathe as needed to keep a pCO2 lower than $20 \mathrm{mmHg}$ for 8 minutes (Maddock and MateoBermudez 1990; Maddock and Carter 1991). Voluntary hyperventilation, unlike inhaling high concentrations of $\mathrm{CO} 2$, leads to respiratory alkalosis where the elimination of $\mathrm{CO} 2$ by the lungs produces an increase in $\mathrm{pH}$ (a decrease in $\mathrm{H}+$ ) in most body fluid compartments and across the blood-brain barrier.

Can voluntary hyperventilation, after an initial respiratory alkalosis, decrease brain $\mathrm{pH}$ as a consequence of the metabolic and/or physicochemical effects of brain lactate elevations? Proton-MRS techniques have shown that panic disorder patients display a disproportionately larger brain lactate response to voluntary hyperventilation than control subjects (Dager, Strauss et al. 1995). This accumulation, due in part to the increased phosphofructokinase (rate-limiting enzyme in glycolysis) activity that results from respiratory alkalosis, usually restores $\mathrm{pH}$ to its normal levels by increasing the levels of an acid ion. It is conceivable that the abnormal lactate response seen in panic disorder disrupts this balance. In work with phosphorous-MRS, pHi decreases were found after 15 minutes into the recovery phase of hyperventilation in healthy volunteers (van Rijen, Luyten et al. 1989). Interestingly, when compared to healthy subjects and patients with other anxiety disorders, panic disorder patients displayed a slower physiological (i.e., pCO2) and psychological recovery after voluntary hyperventilation (Wilhelm, Gerlach et al. 2001). A lagging brain acidosis during this recovery phase can explain the lingering anxious symptoms and low pCO2 (increased ventilation and subsequent low pCO2 would act as compensatory mechanisms to restore $\mathrm{pH}$ ). In a recent phosphorous-MRS study, hyperventilation did not elicit significant differences in $\mathrm{pHi}$ between asymptomatic, medicated panic disorder patients and healthy controls (Friedman, 
Acids in the brain: A factor in panic?

Mathis et al. 2006). However, despite having similar pHi levels, patients with panic disorder maintained lower pCO2 levels than control subjects. Perhaps this was caused by a more acidifying milieu due to increased brain lactate. Also, although no $\mathrm{pHi}$ undershoot was observed during the recovery phase (as in the former phosphorous-MRS study) the reported measurements after 10 minutes were too brief for a direct comparison.

The data presented would seem to suggest that, an acute decrease in brain $\mathrm{pH}$ from baseline levels can occur during the recovery phase after intense hyperventilation. Conceivably, this $\mathrm{pH}$ decrease occurs during an earlier phase when patients panic during the maneuver. Since voluntary hyperventilation is a rather weak and inconsistent panicogen, we presume that brain $\mathrm{pH}$ decreases during this maneuver is an infrequent event, even in susceptible individuals.

\section{Other experimental challenges}

There is an ever-growing number of substances and techniques that are said to provoke panic in panic disorder patients. Because these maneuvers involve various putative modes of action, it has been proposed that panic patients must have an "abnormal fear network" (Gorman, Kent et al. 2000). This altered fear network would overreact to a wide variety of unspecific, arousal-provoking distresses. While arguable, a problem with this view is that many of these challenges, such as those with CCK, flumazenil, caffeine, yohimbine, isoprotenerol, epinephrine, $d$-fenfluramine and mCPP, lack either specificity or sufficient clinical validation to reliably reproduce spontaneous panic attacks in patients with panic disorder (Esquivel, Schruers et al. 2008). Furthermore, the fact that, unlike lactate and $\mathrm{CO} 2$, most of these substances elicit clear hypothalamus-pituitary-adrenal axis activation suggests that the defensive responses they produce are related more to general stress or anxiety than to panic (van Duinen, Schruers et al. 2004; Graeff, Garcia-Leal et al. 2005; van Duinen, Schruers et al. 2005; van Duinen, Schruers et al. 2007).

On the other hand, doxapram administration, though still in need of further validation as a model of panic, may share mechanisms involved in CO2-induced panic. Doxapram may exert its action by inhibiting $\mathrm{pH}$-sensitive potassium channels (Cotten, Keshavaprasad et al. 2006) expressed in brainstem serotonergic neurons (Washburn, Sirois et al, 2002). By inhibiting these channels, doxapram can increase the excitability of brainstem CO2-sensitive neurons.

\section{Mechanisms behind the defensive response to acidosis}

Thus far, we have discussed the mechanisms by which different methods for the experimental provocation of panic can induce brain acidosis. We will now present some potential 
Chapter 8

mechanisms by which acute brain acidosis can elicit defensive reactions in patients with panic disorder and, to a lesser degree, in healthy individuals.

The neural networks of $\mathrm{CO} 2 / \mathrm{H}+$ chemoreception: Beyond respiration

Research into the mechanisms involved in $\mathrm{CO} 2 / \mathrm{H}+$ signaling by specialized neurons has historically been limited to its role in ventilation control. Many regions of the brain that have been found to have intrinsic $\mathrm{CO} 2 / \mathrm{H}+$-sensitive neurons, such as the nucleus tractus solitarii, the medullary raphe, the locus coeruleus, the nucleus ambiguus and the ventrolateral medulla, play a role in ventilation (Putnam, Filosa et al. 2004). Several of these same structures have also been implicated in defensive behavior including panic (Bailey, Argyropoulos et al. 2003). Moreover, recent findings have revealed that midbrain raphe neurons are also $\mathrm{CO} 2 / \mathrm{H}_{+-}$ sensitive (Severson, Wang et al. 2003; Richerson 2004). Severson (2003) has proposed that these midbrain serotonergic neurons with rostral projections, in contrast to those in medullar 5-HT neurons projecting caudally to serve respiration, may be implicated in other behaviors that are also related to respiratory acidosis, such as increased arousal and panic. Orexin hypothalamic neurons, which have a role in the upregulation of arousal and ventilation, are activated by decreases in $\mathrm{pH}$ in a way similar to classical chemosensitive neurons (Williams, Jensen et al. 2007). Other $\mathrm{CO} 2 / \mathrm{H}+$ sensitive neurons, unrelated to acute respiratory control, have also been linked to defensive behavior. The acid-sensing ion channel (ASIC) is widely distributed in the brain and has been implicated in the neuronal activation of non-ventilatory responses to $\mathrm{CO} 2 / \mathrm{H}+$ (Wemmie, Askwith et al. 2003). ASIC 1a, abundantly expressed in the amygdala, appears to mediate fear conditioning in mice as ASIC 1a-null mice displayed deficits in fear conditioning (Wemmie, Askwith et al. 2003) and ASIC 1a-overexpression increased acquired behavior related to fear (Wemmie, Coryell et al. 2004).

The neurons that respond to $\mathrm{CO} 2 / \mathrm{H}+$ and that subsequently drive the cascade of events leading to panic is likely to be a complex system involving many brain sites (Fig. 1). As respiratory symptoms are common complaints in panic (Schruers, van de Mortel et al, 2004; Colasanti, Salamon et al. 2008), particularly in spontaneous panic attacks (Briggs, Stretch et al. 1993), some overlap must exist between chemosensitive neurons that serve respiration and those that elicit panic. For example, the locus coeruleus and hypothalamus are candidate brain locations with $\mathrm{CO} 2 / \mathrm{H}+$-sensitive neurons that most likely serve both respiration (Putnam, Filosa et al. 2004) and defensive responses (Bailey, Argyropoulos et al. 2003; Williams, Jensen et al. 2007). The amygdala, another brain structure implicated in defensive behavior, is also known to have neurons that monitor $\mathrm{CO} 2 / \mathrm{H}+$ (Wemmie, Coryell et al. 2004). Consistent with an implication of many of these chemosensitive areas, a recent neuroimaging investigation with voxel-base morphometry found that panic disorder patients had larger midbrain and rostral pons volumes than controls (Protopopescu, Pan et al. 2006). 


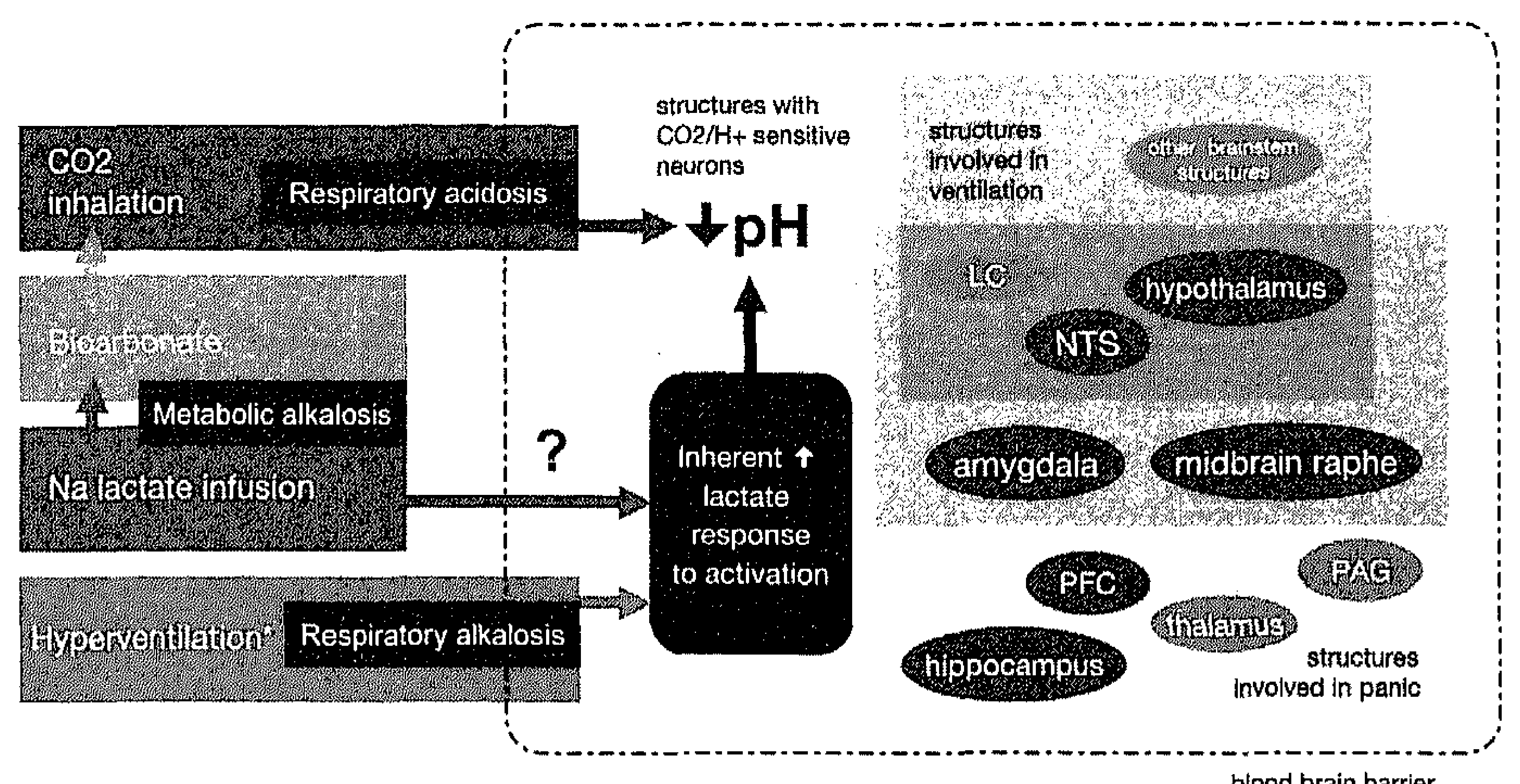

Figure 1. Proposed underlying mechanisms in the defensive response to several experimental challenges. Many brain structures involved in both respiration and defensive behaviors have intrinsic $\mathrm{CO} 2 / \mathrm{H}+$ chemosensitive neurons. Certain characteristics of these types of neurons may render increased vulnerability to disturbances in lactate metabolism and $\mathrm{CO} 2$ inhalations. $\mathrm{CO} 2$ is readily transported in the blood and diffuses easily through the blood-brain barrier. Most infused sodium lactate infused is metabolized in the liver, which results in the simultaneous consumption of $\mathrm{H}+$ and generation of bicarbonate. This will ensue a metabolic alkalosis largely restricted to the intravascular space. How an infusion with sodium lactate leads to an endogenous lactate increase, is still to be explained. "The proposed sequence of events related to acute voluntary hyperventilation is thought to occur during the recovery phase. The produced respiratory alkalosis will increase the activity of phosphofructokinase and subsequently lactate accumulation (exaggerated in the case of patients with panic). Shortly after hyperventilation, the lactate accumulation lagging behind the rising levels $\mathrm{CO} 2$ can produce a decrease in $\mathrm{pH}$

LC: locus ceruleus; NTS: nucleus tractus solitarii; PFC: prefrontal cortex; PAG: periaqueductal grey

Neurons sensitive to increases in $\mathrm{CO} 2 / \mathrm{H}+$ levels have a number of shared characteristics. First, their firing rate increases in response to increased acid (primarily decreased $\mathrm{pHi}$, but also increased $\mathrm{CO} 2$ and/or decreased extracellular $\mathrm{pH}$ ) (Putnam, Filosa et al. 2004). Second, their sensitivity to acid derives from a variety of $\mathrm{pH}$-sensitive ion channels that mediate chemosensitive signaling (which might explain the panicogenic properties of other substances such as doxapram). Third, chemosensitive neurons show a sustained reduction in $\mathrm{pHi}$ in the face of increased extracellular acid load, whereas in other neurons $\mathrm{pHi}$ tends to normalize (Putnam 2001). These shared features of acid-sensitivity across a range of brain regions with differing functions related to respiration, arousal and emotion may be responsible for the ability of threatening disturbances in brain $\mathrm{pH}$ homeostasis, such as that 


\section{Chapter 8}

produced by $\mathrm{CO} 2$ inhalations, to elicit primal responses on a continuum ranging from breathlessness to panic (Liotti, Brannan et al. 2001; Griez, Colasanti et al. 2007).

\section{Vulnerability to panic attacks: A metabolic disturbance?}

What brain mechanisms can explain the unique sensitivity of some individuals, in particular, panic disorder patients, towards challenges in $\mathrm{pH}$ ? In a great of research, panic disorder patients have been shown to have an exaggerated brain lactate response to a variety of metabolic challenges, including sodium lactate infusion (Dager, Marro et al. 1994; Dager, Friedman et al. 1999), voluntary hyperventilation (Dager, Strauss et al. 1995) and visual stimulation (Maddock, Buonocore et al. 2008). This lactate accumulation may explain some findings from voluntary hyperventilation studies, such as the increased alkalotic buffering capacity found in panic disorder patients (Friedman, Mathis et al. 2006) and slight acidosis found in healthy subjects during recovery (van Rijen, Luyten et al. 1989). In several pathological conditions, such as brain lymphoma, increased lactate has also been said to increase respiration and dyspnea by stimulating central $\mathrm{CO} 2 / \mathrm{H}+$ chemoreceptors (Tarulli, Lim et al. 2005; Bluher, Schulz et al. 2008).

This exaggerated brain lactate response can occur during circumstances other than panicogenic maneuvers. In a recent proton-MRS study, panic disorder patients were shown to have larger brain lactate responses to visual stimuli than matched healthy subjects (Maddock, Buonocore et al. 2008). In animal models, it has been shown that discrete brain lactate accumulations can occur during a wide variety and intensity of stressors as weil (Uehara, Sumiyoshi et al. 2008). Furthermore, several neurotransmitter systems affect lactate production in discrete brain areas (Uehara, Sumiyoshi et al. 2008). For example, the administration of benzodiazepine inverse agonists or the induction of experimental stress produced an increase in basolateral amygdaloid nucleus extracellular lactate levels that was attenuated by pretreatment with a benzodiazepine receptor agonist (Uehara, Sumiyoshi et al. 2005). The normal working of the brain is accompanied by lactate production elevations from glucose in astrocytes, lactate is then distributed to neurons where it serves as a substrate in oxidative phosphorylation to support brain activity (Pellerin, Bouzier-Sore et al. 2007). The dispersion/removal of lactate from areas of activation, which is necessary to keep a balanced redox state and cell functioning, is made possible by monocarboxylate transporters which cotransport lactate and $\mathrm{H}+$ from astrocyts to the extracellular fluid and from the extracellular fluid into neurons (Dienel and Hertz 2001). This is consistent with findings where lactate elevations following neuronal activation are associated with extracellular space acidosis (Scheller, Kolb et al. 1992). A recent study focusing on the retrotrapezoid nucleus of the brainstem showed that inhibition of the monocarboxylate transporter responsible for cotransporting lactate and $\mathrm{H}_{+}$ from brain extracellular fluid into neurons leads to decreased extracellular $\mathrm{pH}$ and stimulation 
Acids in the brain: A factor in panic?

of respiration (Erlichman, Hewitt et al. 2008). Many of these or related mechanisms could act on the chemosensitive systems involved in respiration and arousal and mediate the association between increased lactate responses and panic attacks (Maddock, Buonocore et al. 2008).

However, it has not yet been shown that brain $\mathrm{pH}$ changes due to increased brain lactate responses are associated with sensitivity to panic induction by lactate infusions or hyperventilation. In addition, there are some aspects of experimental panic provocation that cannot be easily attributed to increased brain lactate responses. For example, brain lactate increases develop much more slowly than the rapid induction of panic following a single inhalation of CO2. It is possible that the repeated occurrence of spontaneous panic attacks in symptomatic patients with panic disorder leads to a sensitization of the circuits mediating interactions between chemosensitive and fear-related brain regions. In this scenario, elevated brain lactate responses do not directly mediate the panicogenic effect of $\mathrm{CO} 2$ inhalation, but predispose to it indirectly as a result of the sensitizing effects of repeated spontaneous panic attacks triggered, in part, by the activation of chemosensitive neurons.

\section{Discussion}

The defensive response potentially elicited by the rather discrete process of acute brain acidosis can explain a large part of the complex phenotype described in current nosological definitions of panic disorder. The core phenomenon of this heterogeneous disorder are panic attacks (American Psychiatric Association 2000). Current learning theories of panic disorder (Bouton, Mineka et al. 2001) make a clear distinction between panic attacks and anxiety as separable components in a way that is analogous to recent neurobiological views that divide defensive behavior into anxiety and fear (McNaughton and Corr 2004; Graeff and Del-Ben 2008). In these views, which stem from the findings in animal models, anxiety and fear originate in coordinated, but distinct, brain processes that run on a hierarchical continuum from the prefrontal cortex to phylogenically more primitive structures, such as the periaqueductal grey. Higher brain processes account for the complex anxiety (approach) response that is appropriate to deal with distant threats, whereas more primal networks in the brain coordinate the swift and straightforward fear (avoidance) response. This model of anxiety and fear, in clear agreement with human responses to threat (Blanchard, Hynd et al. 2001), has recently gained support from human fMRI experiments (Mobbs, Petrovic et al. 2007) and is thought to have important implications for the etiology of panic (Maren 2007). Conceivably, panic may represent a distinct, primal defensive reaction when the threat is closest, namely, within the body (Fig. 2). 


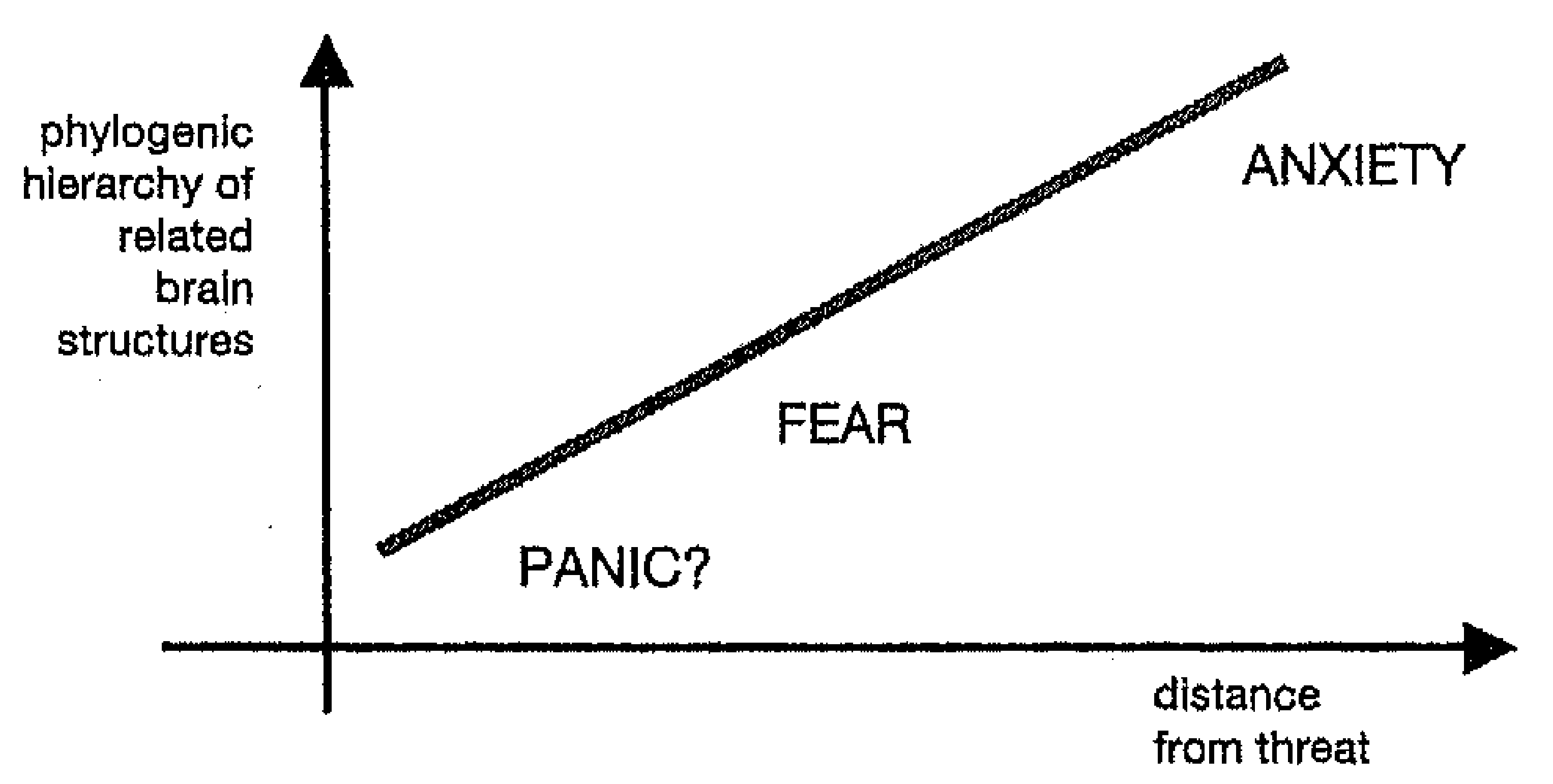

Figure 2. Spontaneous panic as a primal defensive response to the nearest form of threat: acute brain acidosis

Such a course of events is compatible with the theory set forward by Klein (1993), where panic is the result of a suffocation alarm derived from the promotion of a very primitive, survival-oriented behavioral response. Other influential authors have described primal emotional adaptive responses associated with the maintenance of an organism's homeostasis (Panksepp 1998; Damasio 1999; Denton 2006). Such emotions have a singular compelling sensation and intention in which the inducer and the focus of response is mainly the "internal milieu" of the body (Damasio 1999). The evolutionarily inspired value of these is that they indicate that the existence of the organism is being threatened, and they assist in maintaining life by prompting adaptive behaviors. As pointed out by Derek Denton, hunger for air in response to increased $\mathrm{CO} 2$ (or decreased $\mathrm{pH}$ ) is a compelling primal emotion that signals the state of the "suffocating body". Notably, it shares underlying neural substrates (activations of ancient parts of the brain, such as the midbrain, limbic, paralimbic and cerebellar areas) with other primal emotions, like extreme thirst, hunger, pain, micturition, and sleep (Liotti, Brannan et al. 2001).

Extensive research supports the assumption that the degree of brain acidosis is relevant to the panic symptoms elicited by inhalations with $\mathrm{CO} 2$. The question remains whether this $\mathrm{pH}$ disturbance is also relevant in lactate infusions and, to a lesser degree, in voluntary hyperventilation. This model can be explored with the use of proton and phosphorous MRS techniques during experimental panic-provoking paradigms. Using endtidal pCO2 as a covariate, intracellular pH and brain lactate levels can be explored as potential determinants in the symptoms elicited by the different experimental methods to provoke panic. If such is the case, the question also remains whether brain lactate responses are relevant to 
Acids in the brain: A factor in panic?

the experimentally induced symptoms. Measuring brain lactate and pHi during a $5 \%$ or $7 \%$ $\mathrm{CO} 2$ inhalations can provide insight into this matter. Altogether, the results from such studies may explain some of the variance in panic susceptibility that individuals have to voluntary hyperventilation, CO2 inhalations, and a lactate infusion (Gorman, Fyer et al. 1988).

Several methods used to experimentally induce panic attack in susceptible individuals acutely disturb the acid-base homeostasis in the brain. While panic-provoking techniques with $\mathrm{CO} 2$ clearly reduce $\mathrm{pH}$ in all bodily compartments, including the brain, conclusive evidence that this is the case for a sodium lactate infusion and voluntary hyperventilation is still pending. With chemosensitive neurons identified in many brain structures known to be involved in defensive responses, and with the multiple links that panic has with respiration and its determinants, namely $\mathrm{CO} 2 / \mathrm{H}+$ chemoreception, it is conceivable to view panic attacks as a defensive response to acute and potentially threatening acid-base scenarios. We believe this is a useful model, which, if explored, improved, or even refuted, will provide a stable foundation for further progress in our understanding of panic. Panic that is elicited by these provocation maneuvers may very well be the expression of a primal emotion related to brain acidosis. 
Chapter 8

\section{References}

Abelson, J. L., J. G. Weg, et al. (2001). "Persistent respiratory irregularity in patients with panic disorder." Biol Psychiatry 49(7): 588-95.

Abrams, K., Y. Rassovsky, et al. (2006). "Evidence for respiratory and nonrespiratory subtypes in panic disorder." Depress Anxiety 23(8): 474-81.

American Psychiatric Association (2000). Diagnostic and statistical manual of mental disorders: DSM-IVTR. Washington, DC, American Psychiatric Association.

Bailey, J. E., S. V. Argyropoulos, et al. (2003). "Does the brain noradrenaline network mediate the effects of the $\mathrm{CO} 2$ challenge?" J Psychopharmacol 17(3): 252-9.

Blanchard, C. D., A. L. Hynd, et al. (2001). "Human defensive behaviors to threat scenarios show parallels to fear- and anxiety-related defense patterns of non-human mammals." Neurosci Biobehav Rev 25(7-8): 761-70.

Bluher, S., M. Schulz, et al. (2008). "Central lactic acidosis, hyperventilation, and respiratory alkalosis: leading clinical features in a 3-year-old boy with malignant meningeal melanoma." Eur J Pediatr $167(4): 483-5$.

Bouton, M. E., S. Mineka, et al. (2001). "A modern learning theory perspective on the etiology of panic disorder." Psychol Rev 108(1): 4-32.

Briggs, A. C., D. D. Stretch, et al. (1993). "Subtyping of panic disorder by symptom profile." $\mathrm{Br} J$ Psychiatry 163: 201-9.

Cadoux-Hudson, T. A., B. Rajagopalan, et al. (1990). "Response of the human brain to a hypercapnic acid load in vivo." Clin Sci (Lond) 79(1): 1-3.

Caldirola, D., L. Bellodi, et al. (2004). "Approximate entropy of respiratory patterns in panic disorder." Am J Psychiatry 161(1): 79-87.

Carr, D. B. and D. V. Sheehan (1984). "Panic anxiety: a new biological model." J Clin Psychiatry 45(8): 323-30.

Cohen, M. E. and P. D. White (1951). "Life situations, emotions, and neurocirculatory asthenia (anxiety neurosis, neurasthenia, effort syndrome)." Psychosom Med 13(6): 335-57.

Colasanti, A., E. Salamon, et al. (2008). "Carbon dioxide-induced emotion and respiratory symptoms in healthy volunteers." Neuropsychopharmacology 33(13): 3103-10.

Coplan, J. D., T. Sharma, et al. (1992). "Effects of sodium lactate infusion on cisternal lactate and carbon dioxide levels in nonhuman primates." Am J Psychiatry 149(10): 1369-73.

Cotten, J. F., B. Keshavaprasad, et al. (2006). "The ventilatory stimulant doxapram inhibits TASK tandem pore (K2P) potassium channel function but does not affect minimum alveolar anesthetic concentration." Anesth Analg 102(3): 779-85.

Dager, S. R., S. D. Friedman, et al. (1999). "Two-dimensional proton echo-planar spectroscopic imaging of brain metabolic changes during lactate-induced panic." Arch Gen Psychiatry 56(1): 70-7.

Dager, S. R., K. I. Marro, et al. (1994). "Preliminary application of magnetic resonance spectroscopy to investigate lactate-induced panic." Am J Psychiatry 151(1): 57-63.

Dager, S. R., J. M. Rainey, et al. (1990). "Central nervous system effects of lactate infusion in primates." Biol Psychiatry 27(2): 193-204.

Dager, S. R., T. Richards, et al. (1997). "Single-voxel 1H-MRS investigation of brain metabolic changes during lactate-induced panic." Psychiatry Res 76(2-3): 89-99.

Dager, S. R., W. L. Strauss, et al. (1995). "Proton magnetic resonance spectroscopy investigation of hyperventilation in subjects with panic disorder and comparison subjects." Am J Psychlatry 152(5): 666-72.

Dager, S. R., J. B. Yim, et al. (1995). "Application of a novel fiber-optic biosensor in situ to investigate the metabolic effect of lactate infusion." Neuropsychopharmacology 12(4): 307-13.

Dalsgaard, M. K., B. Quistorff, et al. (2004). "A reduced cerebral metabolic ratio in exercise reflects metabolism and not accumulation of lactate within the human brain." J Physiol 554(Pt 2): 571 8.

Damasio, A. R. (1999). The feeling of what happens: body and emotion in the making of consciousness. New York, Harcourt Brace. 
Acids in the brain: A factor in panic?

Denton, D. A. (2006). The primordial emotions: the dawning of consciousness. Oxford; New York, Oxford University Press.

Dienel, G. A. and L. Hertz (2001). "Glucose and lactate metabolism during brain activation." J Neurosci Res 66(5): 824-38.

Erlichman, J. S., A. Hewitt, et al, (2008). "Inhibition of monocarboxylate transporter 2 in the retrotrapezoid nucleus in rats: a test of the astrocyte-neuron lactate-shuttle hypothesis." $J$ Neurosci 28(19): 4888-96.

Esquivel, G., K. Schruers, et al. (2008). Experimental models: panic and fear. Handbook of Anxiety and Fear. R. J. Blanchard, D. C. Blanchard, G. Griebel and D. J. Nutt. Amsterdam, Elsevier B.V. 17: 413-435.

Friedman, S. D., C. M. Mathis, et al. (2006). "Brain pH response to hypenventilation in panic disorder: preliminary evidence for altered acid-base regulation." Am J Psychlatry 163(4): 710-5.

Goetz, R. R., D. F. Klein, et al. (2001). "Acute panic inventory symptoms during CO(2) inhalation and room-air hyperventilation among panic disorder patients and normal controls." Depress Anxiety 14(2): 123-36.

Gorman, J. M., J. Askanazi, et al. (1984). "Response to hyperventilation in a group of patients with panic disorder." Am J Psychiatry 141(7): 857-61.

Gorman, J. M., D. Battista, et al. (1989). "A comparison of sodium bicarbonate and sodium lactate infusion in the induction of panic attacks." Arch Gen Psychiatry 46(2): 145-50.

Gorman, J. M., M. R. Fyer, et al. (1988). "Ventilatory physiology of patients with panic disorder." Arch Gen Psychiatry 45(1): 31-9.

Gorman, J. M., R. R. Goetz, et al. (1990), "Sodium D-lactate infusion of panic disorder patients." Neuropsychopharmacology 3(3): 181-9.

Gorman, J. M., J. M. Kent, et al. (2000). "Neuroanatomical hypothesis of panic disorder, revised." Am J Psychiatry 157(4): 493-505.

Gorman, J. M., L. A. Papp, et al. (1993). "The effect of acetazolamide on ventilation in panic disorder patients." Am J Psychiatry 150(10): 1480-4.

Gorman, J. M. L. A. Papp, et al. (1994). "Anxiogenic effects of CO2 and hyperventilation In patients with panic disorder." Am J Psychiatry 151 (4): 547-53.

Graeff, F. G. and C. M. Del-Ben (2008). "Neurobiology of panic disorder: From animal models to brain neuroimaging." Neurosci Biobehav Rev dol:10.1016/l.neuroblorev.2008.05.017.

Graeff, F. G., C. Garcia-Leal, et al. (2005). "Does the panic attack activate the hypothalamic-pituitaryadrenal axis?" An Acad Bras Cienc 77(3): 477-91.

Griez, E. and K. Schruers (1998). "Experimental pathophysiology of panic." J Psychosom Res 45(6): 493503 .

Griez, E., J. Zandbergen, et al. (1988). "Effects of low pulmonary CO2 on panic anxiety." Compr Psychiatry 29(5): 490-7.

Griez, E. J., A. Colasanti, et al. (2007). "Carbon dioxide inhalation induces dose-dependent and agerelated negative affectivity." PLOS ONE 2(10): e987.

Jensen, K. E., C. Thomsen, et al. (1988). "In vivo measurement of intracellular pH in human brain during different tensions of carbon dioxide in arterial blood. A 31P-NMR study." Acta Physiol Scand 134(2): 295-8.

Klein, D. F. (1993). "False suffocation alarms, spontaneous panics, and related conditions. An integrative hypothesis." Arch Gen Psychiatry 50(4): 306-17.

Kucera, R. R., J. I. Shapiro, et al. (1989), "Brain pH effects of $\mathrm{NaHCO} 3$ and Carbicarb in lactic acidosis." Crit Care Med 17(12): 1320-3.

Liotti, M., S. Brannan, et al. (2001). "Brain responses associated with consciousness of breathlessness (air hunger)." Proc Natl Acad Sci U S A 98(4): 2035-40.

Lum, L. C. (1987). "Hyperventilation syndromes in medicine and psychiatry: a review." J R Soc Med 80(4): 229-31.

Maddock, R. J. (2001). "The lactic acid response to alkalosis in panic disorder: an integrative review." $\underline{\mathrm{J}}$ Neuropsychiatry Clin Neurosci 13(1): 22-34.

Maddock, R. J., M. H. Buonocore, et al. (2008). "Elevated brain lactate responses to neural activation in panic disorder: a dynamic 1H-MRS study," Mol Psychiatry. 


\section{Chapter 8}

Maddock, R. J. and C. S. Carter (1991). "Hyperventilation-induced panic attacks in panic disorder with agoraphobia." Biol Psychiatry 29(9): 843-54.

Maddock, R. J. and J. Mateo-Bermudez (1990). "Elevated serum lactate following hyperventilation during glucose infusion in panic disorder." Biol Psychiatry 27(4): 411-8.

Maren, S. (2007). "Neuroscience. The threatened brain." Science 317(5841): 1043-4.

Martoft, L., H. Stodkilde-Jorgensen, et al. (2003). "CO2 induced acute respiratory acidosis and brain tissue intracellular pH: a 31P NMR study in swine." Lab Anim 37(3): 241-8.

Mathew, R. J., W. H. Wilson, et al. (1989). "Responses to hypercarbia induced by acetazolamide in panic disorder patients." Am J Psychiatry 146(8): 996-1000.

McNaughton, N. and P. J. Corr (2004). "A two-dimensional neuropsychology of defense: fear/anxiety and defensive distance." Neurosci Biobehav Rev 28(3): 285-305.

Mobbs, D., P. Petrovic, et al. (2007). "When fear is near: threat imminence elicits prefrontal-periaqueductal gray shifts in humans," Science 317(5841): 1079-83.

Nakashima, K., T. Yamashita, et al. (1996). "The effect of sodium bicarbonate on CBF and intracellular pH in man: stable Xe-CT and 31P-MRS." Acta Neurol Scand Suppl 166: 96-8.

Nardi, A. E., F. L. Lopes, et al. (2004). "Psychopathological description of hyperventilation-induced panic attacks: a comparison with spontaneous panic attacks." Psychopathology 37(1): $29-35$.

Nardi, A. E., A. M. Valenca, et al. (2004). "Clinical features of panic patients sensitive to hyperventilation or breath-holding methods for inducing panic attacks." Braz J Med Biol Res 37(2): 251-7.

Nardi, A. E., A. M. Valenca, et al. (2006). "Psychopathological profile of 35\% CO2 challenge test-induced panic attacks: a comparison with spontaneous panic attacks." Compr Psychiatry 47(3): 20914.

Nardi, A. E., A. M. Valenca, et al. (2006). "35\% Carbon dioxide and breath-holding challenge tests in panic disorder: a comparison with spontaneous panic attacks." Depress Anxiety 23(4): 236-44.

Nardi, A. E., A. M. Valenca, et al. (2001). "Hyperventilation challenge test in panic disorder and depression with panic attacks." Psychiatry Res 105(1-2): 57-65.

Panksepp, J. (1998). Affective Neuroscience. New York, Oxford University Press.

Papp, L. A., J. M. Martinez, et al. (1997). "Respiratory psychophysiology of panic disorder: three respiratory challenges in 98 subjects." Am J Psychiatry 154(11): 1557-65.

Pellerin, L., A. K. Bouzier-Sore, et al. (2007). "Activity-dependent regulation of energy metabolism by astrocytes: an update." Glia 55(12): 1251-62.

Pitts, F. N., Jr. and J. N. McClure, Jr. (1967). "Lactate metabolism in anxiety neurosis." N Engl J Med 277(25): 1329-36

Protopopescu, X., H. Pan, et al, (2006). "Increased brainstem volume in panic disorder: a voxel-based morphometric study." Neuroreport 17(4): 361-3.

Putnam, R. W. (2001). "Intracellular pH regulation of neurons in chemosensitive and nonchemosensitive areas of brain slices." Respir Physiol 129(1-2): 37-56.

Putnam, R. W., J. A. Filosa, et al. (2004). "Cellular mechanisms involved in $\mathrm{CO}(2)$ and acid signaling in chemosensitive neurons." Am J Physiol Cell Physiol 287(6): C1493-526.

Rassovsky, Y. and M. G. Kushner (2003). "Carbon dioxide in the study of panic disorder: issues of definition, methodology, and outcome." J Anxiety Disord 17(1): 1-32.

Richerson, G. B. (2004). "Serotonergic neurons as carbon dioxide sensors that maintain pH horneostasis." Nat Rev Neuroscl 5(6): 449-61.

Scheller, D., J. Kolb, et al. (1992). "Lactate and pH change in close correlation in the extracellular space of the rat brain during cortical spreading depression." Neurosci Lett 135(1): 83-6.

Schruers, K., T. Klaassen, et al. (2000). "Effects of tryptophan depletion on carbon dioxide provoked panic in panic disorder patients." Psychiatry Res 93(3): 179-87.

Schruers, K. R. J., H. van de Mortel, et al. (2004). "Symptom profiles of natural and laboratory panic attacks." Acta Neuropsychiatrica 16(2): 101-106.

Severson, C. A., W. Wang, et al. (2003). "Midbrain serotonergic neurons are central pH chemoreceptors." Nat Neurosci 6(11): 1139-40.

Shapiro, J. I., M. Whalen, et al. (1989). "Brain pH responses to sodium blcarbonate and Carbicarb during systemic acidosis." Am J Physiol 256(5 Pt 2): H1316-21. 
Acids in the brain: A factor in panic?

Stein, M. B., T. W. Millar, et al. (1995). "Irregular breathing during sleep in patients with panic disorder." Am J Psychiatry 152(8): 1168-73.

Swenson, E. R. and J. M. Hughes (1993). "Effects of acute and chronic acetazolamide on resting ventilation and ventilatory responses in men." J Appl Physiol 74(1): 230-7.

Tarulli, A. W., C. Lim, et al. (2005). "Central neurogenic hyperventilation: a case report and discussion of pathophysiology." Arch Neurol 62(10): 1632-4.

Tsacopoulos, M. and P. J. Magistretti (1996). "Metabolic coupling between glia and neurons." $\underline{J}$ Neurosci 16(3): 877-85.

Uehara, T., T. Sumiyoshi, et al. (2008). "Lactate production and neurotransmitters; evidence from microdialysis studies." Pharmacol Biochem Behav 90(2): 273-81.

Uehara, T., T. Sumiyoshi, et al. (2005). "Enhancement of lactate metabolism in the basolateral amygdala by physical and psychological stress: role of benzodiazepine receptors." Brain Res 1065(1-2): 86-91.

Van den Hout, M. A. and E. Griez (1984). "Panic symptoms after inhalation of carbon dioxide." Br J Psychiatry 144: 503-7.

van Duinen, M. A., K. R. Schruers, et al. (2004). "Hypothalamic-pituitary-adrenal axis function following a $35 \% \mathrm{CO} 2$ inhalation in healthy volunteers." Prog Neuropsychopharmacol Biol Psychiatry 28(2): $279-83$.

van Duinen, M. A., K. R. Schruers, et al. (2005). "CO2 challenge results in hypothalamic-pituitary-adrenal activation in healthy volunteers." J Psychopharmacol 19(3): 243-7.

van Duinen, M. A., K. R. Schruers, et al. (2007). "CO2 challenge induced HPA axis activation in panic." Int J Neuropsychopharmacol 10(6): 797-804.

van Rijen, P. C., P. R. Luyten, et al. (1989). "1H and 31P NMR measurement of cerebral lactate, highenergy phosphate levels, and $\mathrm{pH}$ in humans during voluntary hyperventilation: associated EEG, capnographic, and Doppler findings." Magn Reson Med 10(2): 182-93.

Washburn, C. P., J. E. Sirois, et al. (2002). "Serotonergic raphe neurons express TASK channel transcripts and a TASK-like $\mathrm{pH}$ - and halothane-sensitive $\mathrm{K}+$ conductance." $J$ Neurosci 22(4): $1256-65$.

Wemmie, J. A., C. C. Askwith, et al. (2003). "Acid-sensing ion channel 1 is localized in brain regions with high synaptic density and contributes to fear conditioning." J Neurosci 23(13): 5496-502.

Wemmie, J. A., M. W. Coryell, et al. (2004). "Overexpression of acid-sensing ion channel 1a in transgenic mice increases acquired fear-related behavior." Proc Natl Acad Sci U SA 101(10): 3621-6.

Wilhelm, F. H., A. L. Gerlach, et al. (2001). "Slow recovery from voluntary hyperventilation in panic disorder." Psychosom Med 63(4): 638-49.

Williams, R. H., L. T. Jensen, et al. (2007). "Control of hypothalamic orexin neurons by acid and CO2." Proc Natl Acad Sci U S A 104(25): 10685-90.

Yeragani, V. K., R. K. Radhakrishna, et al. (2002). "Nonlinear measures of respiration: respiratory irregularity and increased chaos of respiration in patients with panic disorder." Neuropsychobiology 46(3): 111-20.

Zandbergen, J., H. H. Lousberg, et al. (1990). "Hypercarbia versus hypocarbia in panic disorder." ل Affect Disord 18(2): 75-81.

Zandbergen, J., H. Pols, et al. (1989). "Effect of hypercapnia and other disturbances in the acid-basebalance on panic disorder." Hillside J Clin Psychiatry 11 (2): 185-97.

Zandbergen, J., H. Pols, et al. (1991). "An analysis of panic symptoms during hypercarbia compared to hypocarbia in patients with panic attacks." $\mathrm{J}$ Affect Disord 23(3): 131-6. 
Summary and concluding remarks 
Our aim in this thesis was to establish and understand the antipanic effects of acute exercise while, at the same time, further our knowledge into the mechanisms of experimental panic. To achieve this, we used $\mathrm{CO} 2$ inhalations as a model to provoke panic and acute exercise as a technique to reduce this panic provocation. We used pharmacological and genetic techniques to explore key neurotransmitter systems and surveyed panic disorder patients on their symptom severity and habitual physical activity. We also updated a model of the mechanisms that underlie experimental panic provocation. Here we summarize our findings and make some concluding remarks.

In chapter 1, as part 1 of the introduction, we reviewed the different methods used to experimentally induce panic (i.e. sodium lactate infusion; $\mathrm{CO} 2$ inhalation; voluntary hyperventilation; cholecystokinin (CCK), doxapram and fumazenil administration; among other methods). For each of theses methods, with an effort to be succinct, we described their background, their characteristic features, their putative mechanisms and, most importantly, their validity as models of panic. The validity of such a model pertains the degree in which the observed pattern in a given method to induce panic corresponds to that of the real life psychopathological process seen in many individuals, especially in patients with panic disorder (PD). The proposed practical criteria required for a valid model of panic include safety, convergence, discrimination, reliability and clinical validity. Under this scope, when these different techniques are revised, several important issues emerge. Apparently, the vulnerability triggered by the different models of panic does not completely overlap, even though they may produce similar symptoms. For instance, with regard to the ability of the model to discriminate between individuals that are affected by PD and those that are not, subjects with generalized anxiety disorder (GAD) or obsessive-compulsive disorder (OCD) are not affected by a $\mathrm{CO} 2$ challenge or a lactate infusion in the same way as PD patients. In contrast, the studies published so far strongly suggest that CCK is as panicogenic in GAD and in OCD as it is in PD. Another issue is that many experimental models are still in need of further validation. Voluntary hyperventilation, for instance, has been recently fairly validated, however the blockability of the symptoms elicited by the maneuver has not been systematically tested with standard anti-panic interventions. Moreover, the evidence so far suggests it is a weak panicogen when compared to $\mathrm{CO} 2$ or lactate. In conclusion, experimental models of panic have proven to be very useful and their further development and refinement may be relevant in the search for intermediate phenotypes linked to genetic and neuroanatomical determinants of panic susceptibility.

Also in chapter 1, as part 2 of the introduction, we reviewed the literature regarding the use of exercise as an evidence-based therapy for affective disorders. A wealth of data supports the use of regular exercise for the prevention and treatment of many illnesses. Recently, exercise has gained increased popularity as a form of therapy for affective disorders. 
Keeping in mind the current paradigm that ranks medical evidence, we looked at results from both epidemiological and experimental studies. Most epidemiological studies show a positive association between levels of physical activity and less affective pathology. Here, the strong associations found in cross-sectional samples are in contrast with the small or nil associations shown in longitudinal studies. A persistent limitation across these samples is that they were not originally intended to address the relationship between exercise and affective symptoms and mostly rely on rather crude data. With regard to experimental studies, many exercise trials have been conducted to treat affective disorders and several reviews and meta-analysis have summarized the findings. Taking these reviews and recent randomized controlled trials in to account, exercise seems to have an effect comparable to standard antidepressive and anxiolytic treatments. Remarkably, despite the great amount of studies, only very few welldesigned trials have been conducted. In summary, the evidence to support the use of exercise as treatment for affective disorders, although encouraging, cannot be considered unequivocal yet. Research in this field is timely.

Chapter 2 describes an experimental study designed to test the antipanic effects of acute exercise on the $35 \% \mathrm{CO} 2$ challenge. Healthy volunteers were randomized to perform either moderate/hard or very-light exercise on a bicycle ergometer and afterwards subjected to a single $35 \% \mathrm{CO} 2$ challenge in a separate group design. We measured the intensity of the symptoms in a panic symptom list (PSL) and the increase of subjective anxiety with a visual analogue scale (NAS-A) before and after the challenge. Compared to the subjects that performed very-light exercise, those that did moderate/hard exercise reported less PSL score increases produced by $\mathrm{CO} 2$. There were no group differences with regard to the score increases on the VAS-A. Intense exercise did not influence pre-challenge VAS-A scores before the challenge, but did increase pre-challenge PSL scores on symptoms that are common to both exertion and panic. Although this latter aspect may potentially have contributed to the findings, we observed no ceiling effect and previous literature on the 35\% $\mathrm{CO} 2$ challenge shows that larger pre-challenge scores has a synergetic rather than an inhibitory effect. With limitations aside, the results suggest that intense exercise has acute antipanic effects in healthy volunteers subjected to a $35 \% \mathrm{CO} 2$ inhalation.

In chapter 3 we report on the results of a study that tested the acute antipanic effects of exercise in patients with $P D$. With the same previous experimental design, in this case participants had current PD as main diagnosis. Subjects did not have comorbidity with more than two psychiatric disorders and were free of any psychotropic medication. Compared to the subjects that performed very-light exercise, those that did moderate/hard exercise reported less PSL and VAS-A score increases induced by $\mathrm{CO} 2$. As in our former study, intense exercise alone did not influence pre-challenge VAS-A scores, but did increase the reported pre-challenge PSL scores on common symptoms to both exertion and panic. Unlike 
Summary and concluding remarks

the former study, moderate/hard exercise was related to smaller increases in both the PSL and VAS-A scores, higher pre-challenge scores could not have contributed to the main findings. These results suggest that intense exercise has acute antipanic in patients with $P D$ when exposed to a $35 \% \mathrm{CO} 2$ inhalation.

Chapter 4 describes a study where that tested the antipanic effects of acute exercise in healthy volunteers subjected to a double-35\% CO2 challenge. Recent data has shown that the CO2-induced affective response is dose-dependent where, unlike lower dosages, a double-inhalation with $35 \% \mathrm{CO} 2$ can evoke symptoms compatible with a panic attack in large proportion of healthy subjects. Accordingly, this study was conducted to determine the viability of testing the effects of exercise on this novel model of panic in healthy participants. On four separate occasions in a randomized Latin square design, healthy volunteers performed either moderate/hard or very-light exercise followed by either a single or a double-35\% $\mathrm{CO} 2$ inhalation. As in our former studies, when subjects performed moderate/hard exercise they reported less PSL and VAS-A score increases produced by both dosages of $\mathrm{CO} 2$. This study confirms our previous findings and those reported independently by others, that intense exercise has an acute anti-panic effect in individuals subjected to high dosages of CO2. Further, as it parallels the antipanic effects seen in patients with PD, it adds to the validity of the $35 \% \mathrm{CO} 2$ inhalation model as panic in healthy subjects.

Chapter 5 describes the first part of a study conducted to test the implication of the endorphinic system in the acute antipanic effects of exercise. Before probing the involvement of the endorphinic system in the effects of acute exercise on $\mathrm{CO} 2$-induced affectivity, we first observed the effects of blocking the opioid system alone on this challenge. Healthy volunteers in a double-blind, randomized, crossover design orally received either naltrexone (a long acting opioid receptor blocker) $50 \mathrm{mg}$ or placebo. Most subjects undertook a $35 \% \mathrm{CO} 2$ doubleinhalation one hour after pre-medication and a separate group did so after 5 hours. Increases in measured symptoms of panic were used as main outcome. Unlike what we expected, opioid blockade after either 1 or 5 hours did not increase the panic response to a doubleinhalation with $35 \%$. On the other hand, naltrexone pre-medication alone elicited significant increments in panic measures. These findings suggest, although preliminarily, that opioid blockade alone can potentially elicit symptoms the resemble panic without modifying the response to $\mathrm{CO} 2$.

In chapter 6, as part of a series of studies on both the underlying mechanisms of $\mathrm{CO} 2$ induced panic and also the implication of the serotonergic system in the acute anti-panic effects of exercise, we report on a study that examined the effects of the 5-HTTLPR genotype in healthy volunteers undergoing different dosages of inhaled $\mathrm{CO} 2$. We expected increases in measured symptoms of panic to be modulated by this genotype. Ninety-six subjects performed a double-inhalation of four mixtures containing respectively $0,9,17.5$ and $35 \%$ 
$\mathrm{CO} 2$, following a randomized, double-blind, cross-over design. Genotype was expressed as LL, SL and SS and was determined using buccal cell material from all participants. Results show that subjects with the SL and SS genotype reported less fear than LL subjects. A significant interaction effect was found between genotype and $\mathrm{CO} 2$ dosage: the SS genotype showed lower fear scores than the LL genotype, particularly in the $17.5 \% \mathrm{CO} 2$ dose condition. The present study demonstrates that the dose-dependent fear reaction to $\mathrm{CO} 2$ is moderated by a polymorphism in the $5-\mathrm{HT}$ transporter gene, particularly at intermediate $\mathrm{CO} 2$ dosages. When one considers that the affective response to the challenge is contingent upon $\mathrm{CO} 2$ dose and individual vulnerability, these results may explain the mixed findings produced by others. They describe the association between the LL allele and stronger responses to a single $35 \%$ $\mathrm{CO} 2$ inhalation in healthy subjects and the lack of any allele associated with the rather large responses to same procedure in patients with $\mathrm{PD}$. This implies that different elements that elicit a strong response to the challenge, high CO2-dose or other aspects of vulnerability, may be such that the modulation 5-HTTLPR genotype is obscured. Our results are in line with current theories on the role of $5-\mathrm{HT}$ in both panic and respiration but also underscore the usefulness of the introduction of an experimental environmental factor to reveal underlying vulnerability in a phenotype, otherwise staying elusive.

in the work detailed in chapter 7 we took our hypothesis out of the lab. We predicted that the involvement in regular exercise would protect individuals against panic attacks. PD patients, whom suffer from frequent panic attacks, were surveyed on their habitual physical activity and different dimensions of panic severity, including panic attacks. In the analysis we controlled for comorbidity, use of antipanic medication, educational level and age as potential confounding factors. With basis in our experimental findings, and those of others, we predicted that lower panic severity scores associated with exercise would be explained, at least in part, by the severity of panic attacks, anticipatory anxiety and disability. Regression coefficients reveal a significant gender by exercise interaction where exercise activity in men was associated with lower panic severity levels. In contrast to our prediction, this association was largely mediated by less disability and agoraphobic avoidance severity and not the frequency of panic attacks per se. Although these findings do not contradict the idea that the benefits of regular exercise in panic disorder patients are related to phenomena we see in our $\mathrm{lab}$, limitations such as the prevalent use of antipanic medication and rather low intensity exercise in our sample make these conclusions preliminary.

In chapter 8, as an afterthought on the biological underpinnings in experimental panic provocation, we propose a model where experimental panic attacks are explained as a primal defensive behavior linked to acute brain acidosis. $\mathrm{CO} 2$ inhalations, lactate infusions and, to a certain extent, voluntary hyperventilation can conceivably lead to a common scenario of brain acidosis in the face of disparate intravascular $\mathrm{pH}$ alterations. While panic-provoking 
techniques with $\mathrm{CO} 2$ clearly reduce $\mathrm{pH}$ in all bodily compartments, including the brain, conclusive evidence that this is the case for a sodium lactate infusion and voluntary hyperventilation is still needs to be further explored. As chemosensitive neurons have been identified in many brain structures involved in defensive responses, and with the multiple links that panic has with respiration and its determinants, namely $\mathrm{CO} 2 / \mathrm{H}+$ chemoreception, it is conceivable to view panic attacks as a defensive response to acute and potentially threatening acid-base scenarios. We believe this is a useful model, which, if explored, improved, or even refuted, will provide a stable foundation for further progress in our understanding of panic.

The work in this thesis has established that exercise has acute antipanic effects. This effect has been replicated by us and independently by others. We have also explored the role of the endorphinic and serotonergic systems in the double-35\% CO2 challenge model as the first step to probe the involvement of these systems in the acute antipanic effects of exercise but also to further our understanding of the underlying mechanisms related to this challenge. In this process we learned that blocking the endorphinc system, by itself, could elicit symptoms that resemble panic, but does not increase the response to the $35 \% \mathrm{CO} 2$ challenge. We also learned that the dose-dependent panic reaction to $\mathrm{CO} 2$ is associated with a polymorphism in the 5-HT transporter gene, particularly at intermediate $\mathrm{CO} 2$ dosages. Subsequent studies with the manipulation of these neurotransmitter systems during acute exercise together with genetic information on the genotypes that modulate their function can shed light into the mechanisms that reduce the reaction to $\mathrm{CO} 2$-induced panic.

As we looked for parallels between experimental findings and cross-sectional associations, preliminary results show a relationship between regular exercise and less severe panic symptoms in men. However, severity and frequency of panic attacks seem not to contribute of this relationship. Beyond the objectives of this study, findings in this crosssectional sample may prove useful for the generation of specific hypotheses to be tested in clinical trials using aerobic exercise in patients with panic disorder. The benefits of aerobic exercise in panic are still to be further demonstrated, characterized and used for the development of neurobiological models in to the mechanisms of acute and sustained antipanic treatment.

Finally, we proposed a model where experimental panic attacks are explained as a primal emotion linked to acute acid-base disturbances. This model can be explored with the use of proton and phosphorous magnetic resonance spectroscopy (MRS) techniques during experimental panic-provoking paradigms. Using end-tidal pCO2 as a covariate, intracellular pH and brain lactate levels can be explored as potential determinants in the symptoms elicited by the different experimental methods to provoke panic. Such work is currently being undertaken in a joint venture with investigators form Ghent University. Panic that is elicited by these 
provocation maneuvers may very well be the expression of a primal emotion related to brain acidosis. 
Samenvatting en conclusies 
Het doel van dit proefschrift is het antipaniek-effect van acute inspanning te staven en er meer inzicht in te krijgen. Een tweede doel is de kennis over de onderliggende mechanismen van experimentele paniek te verbreden. Hiertoe hebben we gebruik gemaakt van $\mathrm{CO} 2$-inhalaties om paniek uit te lokken en acute inspanning om de reactie hierop te reduceren. Verder worden de ernst van de symptomen bij patiënten met een paniekstoornis en hun dagelijkse fysieke activiteit nagegaan. De neurotransmissie-systemen die een sleutelrol toebedeeld krijgen in paniek, worden bestudeerd aan de hand van farmacologische en genetische technieken.

In Hoofdstuk 1 wordt in het eerste gedeelte van de introductie een overzicht gegeven van de verschillende methodes die gebruikt worden om paniek experimenteel te induceren (o.a.: lactaatinfusie, vrijwillige hyperventilatie, cholecystokinine-, of doxapramtoediening, CO2-inhalatie). Voor elke methode worden achtergrond, karakteristieke eigenschappen, en vermoedelijke mechanismen evenals de validiteit als paniekmodel beschreven. De validiteit van een model geeft weer in welke mate het patroon van een bepaalde methode overeenkomt met het werkelijke psychopathologische proces van paniekaanvallen zoals we die zien in paniekpatiënten. De voorgestelde praktische criteria voor een valide paniekmodel zijn: veiligheid, convergentie, discriminatie, betrouwbaarheid en klinische validiteit. Wanneer we de verschillende modellen aan deze criteria toetsen, komen er belangrijke verschilpunten naar voren. Met name de mate van gevoeligheid voor paniekinducie overlapt niet volledig, alhoewel er gelijkaardige symptomen optreden. Zo is de gevoeligheid voor CO2-inhalatie en lactaatinfusie verschillend bij patiënten met een paniekstoornis enerzijds en patiënten met een gegeneraliseerde angststoornis (GAD) of een obesessief-compulsieve stoornis (OCD) anderzijds. Cholecystokinine blijkt echter panicogeen te zijn zowel in paniek patiënten als in GAD en OCD. Uit deze review blijkt dat sommige experimentele modellen nog meer validatie nodig hebben. Inmiddels hebben deze modellen wel bewezen zeer nuttig te zijn en kan de verdere ontwikkeling en verfijning relevant zijn in de zoektocht naar intermediaire fenotypes, gelinkt aan genetische en neuroanatomische determinanten van paniekgevoeligheid.

Het tweede gedeelte van de introductie geeft in hoofdstuk $\mathbf{1}$ een literatuuroverzicht van het gebruik van fysieke inspanning als een evidence-based therapie voor affectieve stoornissen (angst- en stemmingsstoornissen). Fysieke inspanning heeft aan populariteit gewonnen in de behandeling van affectieve stoornissen. Regelmatige lichaamstraining als preventie voor of als behandeling van verschillende aandoeningen wordt dan ook onderbouwd door een scala aan data. In dit overzicht worden zowel epidemiologische als experimentele studies opgenomen. Vanuit de meeste epidemiologische studies wordt er een negatieve associatie aangetoond tussen de mate van fysieke activiteit en psychopathologie. In deze studies staan de sterke associaties in de cross-sectionele studies in 
contrast met de kleine of afwezige associaties in longitudinale studies. Een limitatie van deze studies is echter dat deze er niet specifiek op gericht zijn om de relatie tussen inspanning en affectieve symptomen te onderzoeken, en dus uit gaan van eerder ruwe data. Uit resultaten van experimentele studies blijkt dan weer dat fysieke inspanning een vergelijkbaar therapeutisch effect heeft als standaard antidepressiva en anxiolytica. Ondanks het grote aantal studies zijn er echter weinig goed ontworpen trials uitgevoerd. Samenvattend, er is bewijs dat lichaamsoefening kan gebruikt worden in de behandeling van affectieve stoornissen, maar er is nood aan replicatie.

Hoofdstuk 2 beschrijft een experimentele studie die het antipaniek-effect van acute inspanning onderzoekt. Hierbij ondergaan gezonde vrijwilligers een $35 \%$ CO2-inhalatie na matige/sterke inspanning versus na zeer lichte inspanning. De intensiteit van de symptomen wordt gemeten aan de hand van de 'Panic Symptom List' (PSL) en subjectieve toename in vrees met een visuele analoge schaal (VAS). Diegenen die matige/sterke inspanning uitvoeren vertonen een kleinere toename in symptomen op de PSL dan diegene die zeer lichte inspanning leveren. Er was geen verschil tussen beide groepen op de VAS. Intense inspanning heeft geen effect op de pre VAS-score, maar geeft wel een verhoogde PSL-score voor de inhalatie. Alhoewel dit laatste aspect kan bijdragen aan de bovengenoemde bevindingen, moeten we vaststellen dat er geen plafond-effecten worden waargenomen. Verder blijkt ook uit de literatuur over de CO2-inhalatie dat hogere pre-inhalatie scores eerder een synergistisch dan inhibitorisch effect hebben. De resultaten van deze studie suggereren dus dat intense inspanning een acuut antipaniek-effect heeft op de 35\% CO2-inhalatie in gezonde vrijwilligers.

in hoofdstuk 3 wordt dezelfde studie herhaald in paniekpatiënten. Als exclusiecriteria voor deze patiënten geldt: meer dan twee comorbide psychiatrische aandoeningen en het gebruik van psychotrope medicatie. Patiënten in de groep met matige/sterke inspanning vertonen een kleinere toename zowel op de PSL als op de VAS na de $35 \%$ CO2-inhalatie. Net zoals in hoofdstuk 2 is er geen invloed van inspanning op de pre VAS-scores, maar wel op de PSL. De resultaten suggereren dat er een acuut anti-paniek effect is van intense inspanning in paniekpatiënten wanneer ze blootgesteld worden aan een $35 \%$ CO2-inhalatie.

Hoofdstuk 4 beschrijft een studie waarbij het anti-paniek effect van acute inspanning wordt onderzocht aan de hand van een dubbele $35 \% \mathrm{CO} 2$-inhalatie. Recente data heeft immers aangetoond dat de affectieve respons geïnduceerd door $\mathrm{CO} 2$ dosis afhankelijk is. In tegenstelling tot lagere dosissen kan een dubbele 35\% CO2-inhalatie panieksymptomen uitlokken vergelijkbaar met een paniekaanval in een groot gedeelte van gezonde proefpersonen. Bijgevolg test de studie in hoofdstuk vier of de effecten van acute inspanning ook toepasbaar zijn op het model van de dubbele inhalatie. In een gerandomiseerd "latinsquare' design ondergaan gezonde vrijwilligers op vier verschillende tijdstippen matige/sterke 
of zeer lichte inspanning gevolgd door een éénmalig 35\% CO2-inhalatie of een dubbele 35\% CO2-inhalatie. Net als in de vorige studies zorgt matige/sterke inspanning voor een verminderde toename in symptomen op de PSL en de VAS, zowel bij de éénmalige als bij de dubbele $35 \%$ CO2-inhalatie. Hiermee bevestigt deze studie onze vorige bevinding dat intense inspanning een acuut anti-paniek effect heeft. Daarenboven correspondeert het resultaat met het anti-paniek effect in paniekpatiënten. Dit draagt bij tot de validiteit van het $35 \% \mathrm{CO} 2$ inhalatie als model voor paniek in gezonde vrijwilligers.

In hoofdstuk 5 wordt een deelstudie beschreven over de relatie tussen het endorfine systeem en het anti-paniek effect van fysieke inspanning. In deze studie kijken we eerst welk effect het blokkeren van het opioide systeem heeft op de CO2-inhalatie (In een eventuele volgende studie zal de factor inspanning toegevoegd worden) in een dubbel-blind gerandomiseerd crossover design krijgen gezonde vrijwilligers een orale dosis van $50 \mathrm{mg}$ naltrexone of placebo toegediend gevolgd door een dubbele $35 \% \mathrm{CO}$-inhalatie. Tegen de verwachting in leidt een blokkade van opioide systeem niet tot een verhoogde paniekreactie in respons op de $\mathrm{CO} 2$-inhalatie. Echter, naltrexone premedicatie leidt op zichzelf tot een significante stijging in paniek maten. De bevindingen van deze studie, alhoewel preliminair, suggereren dat een blokkade van het opioide systeem potentieel symptomen kan opwekken die lijken op paniek zonder echter de respons op de CO2-inhalatie te veranderen.

Hoofdstuk 6 maakt deel uit van een serie studies die zowel de onderliggende mechanismen van $\mathrm{CO} 2$-geïnduceerde paniek bestuderen als de implicatie van het serotonerge systeem in het acuut anti-paniek effect van intense inspanning. In deze studie wordt het effect van het 5-HTTLPR genotype (LL, SL, SS) onderzocht op de inhalatie van verschillende dosissen $\mathrm{CO} 2(0,9,17.5,35 \%)$ in gezonde vrijwilligers. Deze studie toont aan dat individuen met het SL en het SS genotype minder vrees rapporteren dan de het LL genotype. Verder wordt er een significant interactie effect gevonden tussen het genotype en de CO2 dosis: het SS genotype vertoont lagere vrees-scores dan het $L L$ type, het meest uitgesproken bij de dosis van $17.5 \%$. Deze studie toont dus aan dat de dosisafhankelijke reactie op de dubbele CO2-inhalatie gemodereerd wordt door een polymorfisme in het gen voor de $5 \mathrm{HT}$-transporter, vooral bij intermediaire dosissen. Wanneer men in overweging neemt dat de affectieve respons op de CO2-inhalatie afhankelijk is van de dosis en de individuele kwetsbaarheid, kan men schijnbaar tegenstrijdige bevindingen van anderen verklaren. De resultaten van onze studie zijn niet enkel in lijn met de huidige theorieën over de rol van serotonine in paniek en respiratie, maar onderlijnen ook het nut van de introductie van een experimentele omgevingsfactor om de onderliggende kwetsbaarheid van een fenotype aan het licht te brengen.

In het volgende hoofdstuk zetten we een stap buiten het lab. In hoofdstuk 7 stellen we de hypothese dat het regelmatig beoefenen van sport individuen beschermt tegen paniekaanvallen. Om dit te onderzoeken werden mensen met een paniekstoornis ondervraagd 
over hun gewoonten inzake fysieke activiteit en over verschillende dimensies van de ernst van de paniek, inclusief de paniekaanvallen. In de analyses wordt er gecontroleerd voor comorbiditeit, het gebruik van medicatie, educatie niveau en leeftijd. Regressiecoëfficiënten tonen een significantie interactie tussen geslacht en fysieke inspanning waarbij het beoefenen van sport in mannen geassocieerd is met lagere paniekscores. Deze associatie blijkt voornamelijk gemedieerd te worden door beperkingen in het dagelijkse leven en de mate van vermijdingsgedrag, maar niet door de frequentie van paniekaanvallen per se. Deze bevindingen ondersteunen het idee dat de gunstige effecten van regelmatige lichaamsbeweging in paniekpatiënten gerelateerd zijn aan fenomenen die we zien in de experimentele setting. Doordat in deze studie veelvuldig sprake was van gebruik van antipaniek medicatie en omdat de gerapporteerde intensiteit van lichaamsbeweging eerder licht was zijn de conclusies slechts preliminair te noemen.

Hoofdstuk 8 presenteert een nabeschouwing over de onderliggende biologische mechanismen in experimentele paniekprovocatie. Hierin wordt een model naar voren gebracht waarin experimentele paniekaanvallen verklaard worden als primair defensief gedrag gelinkt aan acute acidose in de hersenen. Men kan een gemeenschappelijk scenario bedenken waarbij de CO2-inhalatie, lactaatinfusie en tot op zekere hoogte ook de vrijwillige hyperventilatie mogelijk kunnen leiden tot acidose in de hersenen ondanks tegenovergestelde veranderingen in intravasculaire $\mathrm{pH}$. De paniekprovocerende technieken met $\mathrm{CO} 2$ reduceren duidelijk de $\mathrm{pH}$ in alle lichaamscompartimenten, ook in de hersenen. Er moet nog verder onderzocht worden of dit ook het geval is bij lactaatinfusie en vrijwillige hyperventilatie. Er zijn chemosensitieve neuronen geïdentificeerd in verschillende hersenstructuren die betrokken zijn bij onze defensieve reacties. Daarenboven wordt paniek veelvuldig gelinkt met respiratie en zijn determinanten, zoals CO2/H+ chemoreceptie. Dit maakt het denkbaar dat paniekaanvallen een defensieve reactie zijn op een acute en potentieel bedreigende verstoring in de zuur-base balans.

De studies in het huidig proefschrift hebben aangetoond dat fysieke inspanning een acuut anti-paniek effect heeft. Dit effect is gerepliceerd door onszelf en ook onafhankelijk door anderen. We hebben de rol van het endorfine en het serotonerge systeem in de reactie op de $\mathrm{CO} 2$ geëxploreerd als een eerste stap naar hun betrokkenheid in het acute anti-paniek effect. Hieruit hebben we geleerd dat een blokkade van het endorfine systeem leidt tot panieksymptomen, maar dat het de reactie op de CO2-inhalatie niet versterkt. Verder hebben we ook aangetoond dat de dosisafhankelijke respons op de inhalatie gemodereerd wordt door een polymorfisme in het gen voor de serotonine transporter, vooral bij intermediaire doses. In de toekomst zullen studies die deze neurotransmissiesystemen manipuleren tijdens acute fysieke inspanning gecombineerd met genetische informatie meer inzicht geven in de onderliggende mechanismen van de gereduceerde $\mathrm{CO} 2$-geinduceerde paniekreactie. 
Wanneer we kijken naar parallellen tussen de experimentele setting en crosssectienele associaties, tonen preliminaire resultaten een relatie aan tussen regelmatige lichaamsbeweging en minder ernstige panieksymptomen in mannen. De bevindingen in dit cross-sectionele sample kunnen aangewend worden voor het genereren van hypothesen omtrent aerobe inspanning in paniekpatiënten en die vervolgens kunnen getest worden in klinische trials. De voordelen van aerobe inspanning in paniek moeten verder onderzocht worden. Vervolgens kan dit gebruikt worden voor de ontwikkeling van neurobiologische modellen die het mechanisme van het acute en aanhouden anti-paniek effect verklaren.

Om te eindigen stellen we een model voor waarbij experimentele paniekaanvallen verklaard worden als een primaire emotie gelinkt aan verstoringen in de zuur-base balans. Dit model kan getoetst worden aan de hand van proton en fosfor magnetische resonantie spectroscopie (MRS) technieken gecombineerd met experimentele paniekprovocatie. Wanneer we de end-tidal $\mathrm{CO} 2$ gebruiken als covariaat, kunnen de intracellulaire $\mathrm{pH}$ en het lactaatniveau in de hersenen geëxploreerd worden als potentiële determinanten in de symptomen bij experimentele paniek. Zulke studies zijn reeds gestart in samenwerking met de universiteit van Gent. 


\section{Acknowledgments}

This is a labor of tove, but it would have not been possible without a great deal of help from the many people to whom I owe an acknowledgement.

First of all, I must thank my mentors Prof.dr. Eric Griez and Dr. Koen Schruers. I have been very fortunate to learn about research and its intricacies from such dedicated and passionate scientists. The kind opportunity from Prof. Griez to experience research from conception to publication during an internship as a medical student had a particularly profound influence. I also have special gratitude to Dr. Schruers for sharing with me his enthusiasm for food and wine as well as for neuroscience. For their generous and engaging mentorship I will always be grateful.

Key components of this thesis were made possible by the guidance, support and partnership from many outstanding experts. Much appreciation goes to Prof.dr. Harm Kuipers and Dr. Fred Hartgens for their advice and collaboration with expertise in sports medicine. I warmly acknowledge my many mentors and fellow colleagues from the Instituto Nacional de Psiquiatría "Ramón de la Fuente Muñiz" for all their help, particularly Dr. Carlos Berlanga, Dr. Carmen Lara and Dr. J. Leonardo Diaz for their involvement in this project. I must also thank Prof.dr. Richard Maddock from the University of California Davis for his keen and timely collaboration with expertise in acid-base physiology and panic.

I have a very special appreciation for the collaborators that have spent long hours with experiments and data collection. I must thank Loes Wijnhoven and Malou Pereboom for their dedication, patience and contribution to the work in this thesis. I also thank Oscar FernándezTorre and Abdulkader Dandachi for their contribution, but also for their warm friendship and rich Spanish and Syrian folklore!

To many close colleagues I have a lot to thank for. Especially to Lies Goossens for her friendship and so many hours of help with the worst kafkanian bureaucratic tasks. Marlies van Duinen for her experienced opinion on so many occasions (to her 3 year old son, Lars, who has patiently tried to teach me some Dutch, bedankt!). Leni Noteborn for unquantifiable amount of help with just about everything. Inge Knuts for her company and countless moments of advice and laughter. Much gratitude goes to Rob van Diest for the answers to my many questions (and the many more interesting answers to questions I had not though of). My colleagues from the Academic Anxiety Center Inge Lensing, Julien Peeters, Klara de Cort, Thea Overbeek and Judith Rickelt for all the support. I thank Alessandro Colasanti for his kind 
Acknowledgments

collaboration (and for sharing some real Italian food). I am also in great debt with my brilliant colleagues at the section of Social Psychiatry who have always been keen to help me with my questions. I specially thank Marieke Wichers and Marjan Drukker for their advice with statistics.

Ultimately, I owe all in this work to the many volunteers from Maastricht and Mexico that participated in the studies that conform this thesis. I am particularly grateful to the brave patients with panic disorder who, despite their affection, were always eager to contribute.

I have also been privileged with the affection and guidance of many wonderful people who are also, in one way or another, ingredients to this milestone. I thank my many Dutch friends for helping me adjust to their great country. Le agradezco a mis maestros y amigos por buena parte de la inspiración y motivación para realizar esta tesis. Mención muy especial merece mi familia por su cariño y constante estímulo. Finally, I thank Tiny for proofreading just about every chapter in this thesis and for making life much more organized.

Gabriel Esquivel 


\section{Curriculum vitae}

Gabriel Esquivel was born on December 9, 1972 in Chicago Illinois. At the age of 8 he then lived in Mexico. In 1993 he completed baccalaureate education and then followed architecture school in Monterrey and Mexico City. After 2 summer internships in an architectural firm he was convinced of a necessary shift in career. In 1996 he started medical school in Mexico City's Universidad Autónoma Metropolitana and graduated as a physician 6 years later. From 2002 to 2006 he trained as a specialist in adult psychiatry at the Instituto Nacional de Psiquiatría Ramón de la Fuente Muñiz and Universidad Nacional Autónoma de México. Over the course of his medical and specialist training he also undertook the semi-residential International Master in Affective Neuroscience course from Maastricht University/University of Florence and graduated in 2004. He began research work in 2006, under continued mentorship from Prof.Dr. Eric Griez and Dr. Koen Schruers, towards a PhD degree. He is currently involved in research and in the planning group for the International Track in Medicine at the Department of Psychiatry \& Neuropsychology, Maastricht University. 


\section{Publication list}

Wi-1: Scientific publication in international journal mentioned in the Social Science Citation Index, Science Citation Index or Arts \& Humanities Citation Index with Impact Factor.

Esquivel G, Schruers K, Kuipers $H$, Griez E (2002) The effects of acute exercise and high lactate levels on $35 \% \mathrm{CO} 2$ challenge in healthy volunteers. Acta Psychiatr Scand 106: 394-397

Esquivel G, Diaz-Galvis J, Schruers K, Berlanga C, Lara-Munoz C, Griez E (2008) Acute exercise reduces the effects of a $35 \% \mathrm{CO}(2)$ challenge in patients with panic disorder. $J$ Affect Disord 107: 217-220

Esquivel G, Fernández-Torre O, Schruers KRJ, Wijnhoven LLW, Griez EJL (2008) The effect of opioid receptor blockade on experimental panic provocation with $\mathrm{CO} 2 . \mathrm{J}$ Psychopharmacol Ahead of print: 1-4

Lim L, Temel Y, Vissar-Vanderwalle V, Steinbusch H, Schruers K, Hameleers R, Esquivel G, Griez E, Blokland A (2008) Effect of buspirone on the behavioral regulation of rats in low versus high anxiety conditions. Arzneimittel-Forschung (Drug Research) 58: 269-276

Esquivel G, Schruers K, Maddock RJ, Colasanti A, Griez E (2009) Acids in the brain. A factor in panic? J Psychopharmacol (Accepted)

WN: Scientific publication in national journal with referee system.

Esquivel G, Hartgens F, Schruers K, Griez E (2008) [Physical exercise as (adjuvant) treatment for depression and anxiety disorders]. Stimulus 27: 90-107

Jimenez-Genchi A, Monteverde-Maldonado E, Nenclares-Portocarrero A, Esquivel-Adame G, de la Vega-Pacheco A (2008) [Reliability and factorial analysis of the Spanish version of the Pittsburg Sleep Quality Index among psychiatric patients]. Gac Med Mex 144: $491-496$ 
Publication list

WBMBC: Scientific publication as a book or as a contribution to a book including proceedings.

Schruers K, Esquivel G, Kuipers H, Griez E (2002) The antipanic effects of acute exertion and high exertion on $35 \% \mathrm{CO} 2$ challenge in healthy subjects. European Neuropsychopharmacology 12: S352-S352

Esquivel G, Berlanga-Cisneros C, Lara-Munoz C, Griez E (2006) The effects of exertion and high levels of lactate in a $35 \% \mathrm{CO} 2$ challenge in patients with panic disorder. European Neuropsychopharmacology 16: S81-S82

Saeed NP, Esquivel G, Graf IM, Hoeks APG, van Duinen MA (2007) Response of Blood Flow and Vascular Resistance in Common Carotid Artery to Hypercapnia. Artery Research 1: 48-48

Esquivel G, Schruers K, Griez E (2008) Experimental models: panic and fear. In: R.J. Blanchard DCB, G. Griebel and D.J. Nutt (ed) Handbook of Anxiety and Fear. Elsevier B.V., pp 413-435

Esquivel G, Fernández-Torre O, Schruers K, Wijnhoven LLW, Griez E (2008b) The effects of acute naltrexone premedication in experimental panic provocation. European Neuropsychopharmacology 18: S497-S498

Saeed NP, Esquivel G, Graf IM, Hoeks APG (2008) Influence of age on carotid endothelial function as determined by hypercapnia-induced vasodilatation. Artery Research 2: 98-99 